\title{
ADVANCES IN TRANSORAL APPROACHES FOR LARYNGEAL CANCER
}

EDITED BY: Cesare Piazza, Giorgio Peretti and Vincent Vander Poorten PUBLISHED IN: Frontiers in Oncology

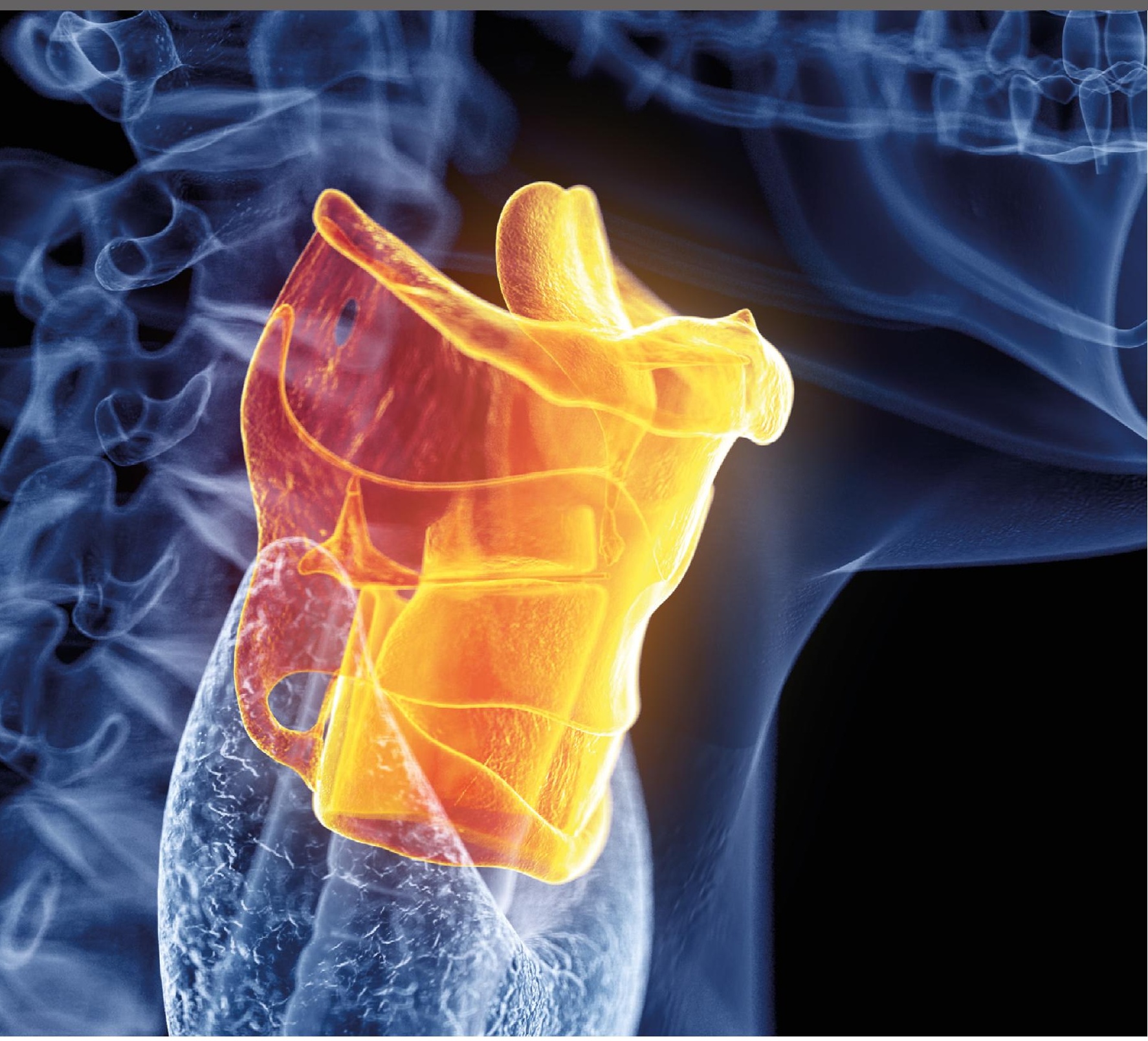

frontiers Research Topics 


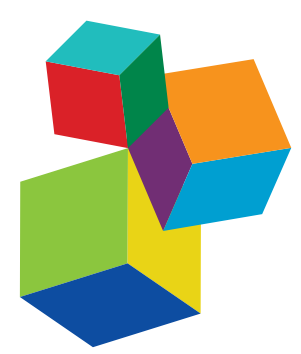

Frontiers Copyright Statement

C Copyright 2007-2019 Frontiers Media SA. All rights reserved.

All content included on this site, such as text, graphics, logos, button

icons, images, video/audio clips, downloads, data compilations and

software, is the property of or is

licensed to Frontiers Media SA

("Frontiers") or its licensees and/or subcontractors. The copyright in the text of individual articles is the property of their respective authors, subject to a license granted to Frontiers.

The compilation of articles constituting this e-book, wherever published, as well as the compilation of all other content on this site, is the exclusive property of Frontiers. For the

conditions for downloading and copying of e-books from Frontiers website, please see the Terms for Website Use. If purchasing Frontiers e-books from other websites or sources, the conditions of the website concerned apply.

Images and graphics not forming part of user-contributed materials may not be downloaded or copied without permission

Individual articles may be downloaded and reproduced in accordance with the principles of the CC-BY

licence subject to any copyright or other notices. They may not be re-sold as an e-book.

As author or other contributor you grant a CC-BY licence to others to reproduce your articles, including any graphics and third-party materials supplied by you, in accordance with the Conditions for Website Use and subject to any copyright notices which you include in connection with your articles and materials.

All copyright, and all rights therein, are protected by national and international copyright laws.

The above represents a summary only.

For the full conditions see the Conditions for Authors and the Conditions for Website Use.

ISSN 1664-8714

ISBN 978-2-88945-777-9

DOI 10.3389/978-2-88945-777-9

\section{About Frontiers}

Frontiers is more than just an open-access publisher of scholarly articles: it is a pioneering approach to the world of academia, radically improving the way scholarly research is managed. The grand vision of Frontiers is a world where all people have an equal opportunity to seek, share and generate knowledge. Frontiers provides immediate and permanent online open access to all its publications, but this alone is not enough to realize our grand goals.

\section{Frontiers Journal Series}

The Frontiers Journal Series is a multi-tier and interdisciplinary set of open-access, online journals, promising a paradigm shift from the current review, selection and dissemination processes in academic publishing. All Frontiers journals are driven by researchers for researchers; therefore, they constitute a service to the scholarly community. At the same time, the Frontiers Journal Series operates on a revolutionary invention, the tiered publishing system, initially addressing specific communities of scholars, and gradually climbing up to broader public understanding, thus serving the interests of the lay society, too.

\section{Dedication to Quality}

Each Frontiers article is a landmark of the highest quality, thanks to genuinely collaborative interactions between authors and review editors, who include some of the world's best academicians. Research must be certified by peers before entering a stream of knowledge that may eventually reach the public - and shape society; therefore, Frontiers only applies the most rigorous and unbiased reviews.

Frontiers revolutionizes research publishing by freely delivering the most outstanding research, evaluated with no bias from both the academic and social point of view. By applying the most advanced information technologies, Frontiers is catapulting scholarly publishing into a new generation.

\section{What are Frontiers Research Topics?}

Frontiers Research Topics are very popular trademarks of the Frontiers Journals Series: they are collections of at least ten articles, all centered on a particular subject. With their unique mix of varied contributions from Original Research to Review Articles, Frontiers Research Topics unify the most influential researchers, the latest key findings and historical advances in a hot research area! Find out more on how to host your own Frontiers Research Topic or contribute to one as an author by contacting the Frontiers Editorial Office: researchtopics@frontiersin.org 


\section{ADVANCES IN TRANSORAL APPROACHES FOR LARYNGEAL CANCER}

Topic Editors:

Cesare Piazza, University of Milan, Italy

Giorgio Peretti, University of Genoa, Italy

Vincent Vander Poorten, University Hospitals Leuven and KU Leuven, Belgium

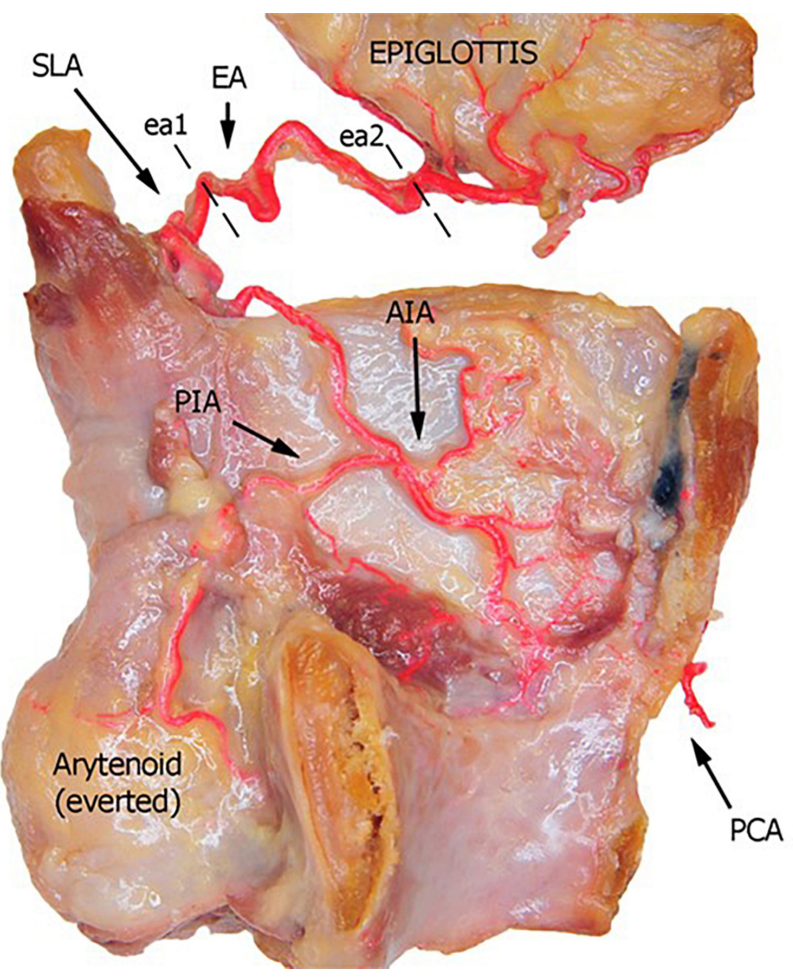

Left fresh-frozen cadaver hemilarynx after midline section of the cricoid and thyroid cartilages, eversion of the ipsilateral arytenoid, and removal of the left aryepiglottic, false, true vocal folds, and paraglottic fat tissue, thus exposing the inner thyroid lamina. Bicomponent red silicone had been injected in the left common and subclavian arteries. Dotted lines ea1 and ea2 represent the landmark points used for measurements of the EA. Abbreviations: SLA, superior laryngeal artery; EA, epiglottic artery; AIA antero-inferior artery; PIA, postero-inferior artery; PCA, paracommissural branch of the crico-thyroid artery. From: Perotti P, Ferrari M, Montalto N Lancini D, Paderno A, Incandela F, Buffoli B, Rodella LF and Piazza C (2018) Anatomical Cadaver Study of Endolaryngeal Vascularization: Focus on the Glottis, Supraglottis, and Subglottis From the Transoral Microsurgical Point of View. Front. Oncol. 8:138. doi: 10.3389/fonc.2018.00138

Cover image: Sebastian Kaulitzki/Shutterstock.com 
We live in an era of increasing availability of highly technological tools that allow for reducing, where possible, the invasiveness of the surgical procedures needed to remove cancer.

Also in the head and neck area, transoral procedures increasingly replace the traditional, more invasive, open access procedures. Crucial developments promoting this evolution were the popularization of transoral laser microsurgery and, more recently, transoral robotic approaches. It is logical to expect that these approaches will entail better function for the patients, and thus better quality of life, provided that the obtained oncological radicality is the same or better than that obtained with the more invasive procedures of the past. All these "logical" assumptions still need confirmation and validation on large and independent series, however.

This eBook collects the results of some of the most recent research efforts in this field. It contains studies aimed at advancing surgical technology and oncological philosophy, as well as studies assessing the oncological as well as the functional outcome following the application of these techniques. In this way this e-book aims at critically assessing and, where justified, boosting the development and popularization of these transoral approaches. The overall aim is evidently to improve both the oncological as well as the functional outcomes for the patient with laryngeal cancer.

Citation: Piazza, C., Peretti, G., Vander Poorten, V., eds. (2019). Advances in Transoral Approaches for Laryngeal Cancer. Lausanne: Frontiers

Media. doi: 10.3389/978-2-88945-777-9 


\section{Table of Contents}

06

09

17

Editorial: Advances in Transoral Approaches for Laryngeal Cancer Cesare Piazza, Giorgio Peretti and Vincent Vander Poorten Laryngological Society Laser Cordectomy Classification

Abie H. Mendelsohn and Marc Joseph Remacle Improvements in High Resolution Laryngeal Magnetic Resonance Imaging for Preoperative Transoral Laser Microsurgery and Radiotherapy Considerations in Early Lesions

Thomas Ruytenberg, Berit M. Verbist, Jordi Vonk-Van Oosten, Eleftheria Astreinidou, Elisabeth V. Sjögren and Andrew G. Webb

25 Single Stage Transoral Laser Microsurgery for Early Glottic Cancer Yaniv Hamzany, Hagit Shoffel-Havakuk, Stav Devons-Sberro, Shani Shteinberg, Dan Yaniv and Aviram Mizrachi

33 Impact of Close and Positive Margins in Transoral Laser Microsurgery for Tis-T2 Glottic Cancer

Ivana Fiz, Francesco Mazzola, Francesco Fiz, Filippo Marchi, Marta Filauro, Alberto Paderno, Giampiero Parrinello, Cesare Piazza and Giorgio Peretti

42 Up-Front and Salvage Transoral Laser Microsurgery for Early Glottic Squamous Cell Carcinoma: A Single Centre Retrospective Case Series Jeroen Meulemans, Jacqueline Bijnens, Pierre Delaere and Vincent Vander Poorten

53 Three-Dimensional Map of Isoprognostic Zones in Glottic Cancer Treated by Transoral Laser Microsurgery as a Unimodal Treatment Strategy Cesare Piazza, Marta Filauro, Alberto Paderno, Filippo Marchi, Pietro Perotti, Riccardo Morello, Stefano Taboni, Giampiero Parrinello, Fabiola Incandela, Andrea landelli, Francesco Missale and Giorgio Peretti

$60 \mathrm{CO}_{2}$ Transoral Microsurgery for Supraglottic Squamous Cell Carcinoma Filippo Carta, Cinzia Mariani, Giovanni B. Sambiagio, Natalia Chuchueva, Elisa Lecis, Clara Gerosa and Roberto Puxeddu

71 Transoral Laser Microsurgery for Supraglottic Cancer Petra Ambrosch, Mireia Gonzalez-Donate, Asita Fazel, Claudia Schmalz and Jürgen Hedderich

81 High Frequency Jet Ventilation During Transoral Laser Microsurgery for Tis-T2 Laryngeal Cancer

Francesco Mora, Francesco Missale, Fabiola Incandela, Marta Filauro, Giampiero Parrinello, Alberto Paderno, Palmiro Della Casa, Cesare Piazza and Giorgio Peretti

86 Anatomical Cadaver Study of Endolaryngeal Vascularization: Focus on the Glottis, Supraglottis, and Subglottis From the Transoral Microsurgical Point of View

Pietro Perotti, Marco Ferrari, Nausica Montalto, Davide Lancini, Alberto Paderno, Fabiola Incandela, Barbara Buffoli, Luigi Fabrizio Rodella and Cesare Piazza 
93 A Contemporary Review of Evidence for Transoral Robotic Surgery in Laryngeal Cancer

Philippe Gorphe

100 Management of the Airway for Transoral Robotic Supraglottic Partial Laryngectomy

Vanessa C. Stubbs, Karthik Rajasekaran, Adam R. Gigliotti, Ahmad F. Mahmoud, Robert M. Brody, Jason G. Newman, Christopher H. Rassekh and Gregory S. Weinstein

106 Transoral Robotic Surgery in the Nordic Countries: Current Status and Perspectives

Antti A. Mäkitie, Harri Keski-Säntti, Mari Markkanen-Leppänen, Leif Bäck, Petri Koivunen, Tomas Ekberg, Karl Sandström, Göran Laurell,

Mathias von Beckerath, Johan S. Nilsson, Peter Wahlberg, Lennart Greiff, Lena Norberg Spaak, Thomas Kjærgaard, Christian Godballe, Oddveig Rikardsen, Hani Ibrahim Channir, Niclas Rubek and Christian von Buchwald 


\section{OPEN ACCESS}

Edited and reviewed by: Andreas Dietz,

Leipzig University, Germany

*Correspondence: Cesare Piazza

cesare.piazza@istitutotumori.mi.it; ceceplaza@libero.it

Specialty section:

This article was submitted to

Head and Neck Cancer,

a section of the journal

Frontiers in Oncology

Received: 25 September 2018 Accepted: 28 September 2018 Published: 17 October 2018

Citation:

Piazza C, Peretti G and Vander Poorten V (2018) Editorial: Advances in Transoral Approaches for

Laryngeal Cancer.

Front. Oncol. 8:455.

doi: 10.3389/fonc. 2018.00455

\section{Editorial: Advances in Transoral Approaches for Laryngeal Cancer}

\author{
Cesare Piazza ${ }^{1 *}$, Giorgio Peretti ${ }^{2}$ and Vincent Vander Poorten ${ }^{3,4}$ \\ 1 Department of Otorhinolaryngology, Maxillofacial, and Thyroid Surgery, Fondazione IRCCS National Cancer Institute of \\ Milan, University of Milan, Milan, Italy, ${ }^{2}$ Department of Otorhinolaryngology - Head and Neck Surgery, University of Genoa, \\ Genoa, Italy, ${ }^{3}$ Department of Otorhinolaryngology - Head and Neck Surgery, University Hospital Leuven, Leuven, Belgium, \\ ${ }^{4}$ Section Head and Neck Oncology, Department of Oncology, KU Leuven, Leuven, Belgium
}

Keywords: transoral access, laser surgery, laryngeal cancer, robotic surgery, minimally invasive surgery

\section{Editorial on the Research Topic}

\section{Advances in Transoral Approaches for Laryngeal Cancer}

If the reader googles words such as "transoral laser (or robotic) surgery for laryngeal cancer," he will quickly realize how such relatively new topics are among the most actively discussed in the recent years by the international head and neck cancer community. Whether this is truly (and exclusively) the result of the publication in 1993 of Steiner's first large series of glottic tumors treated by transoral laser microsurgery (TLM) (1) and the introduction of the European Laryngological Society (ELS) classification scheme of endoscopic cordectomies in 2000 (2) as hypothesized by Mendelsohn and Remacle, can be a matter of debate. History will tell: as in all human developments, longer followup time and assessment by judges that are at some distance are needed to properly evaluate the historical roots of such a complex scientific, economic, and social phenomenon. However, no one can forget the value of such seminal papers and their groundbreaking effect when first published in the last century. Once the revolutionary Steiner's concept of multi-bloc trans-tumor resection of large volume laryngo-hypopharyngeal malignancies was demonstrated to not hamper any of the mainstays of oncologic radicality, given that an adequate preoperative three-dimensional (3D) model of the tumor to be removed had been fully understood, a number of "traditional" surgeons (or their younger scholars) gradually shifted their indications for management of laryngeal cancer from open to transoral approaches.

In this sense, minimally invasive surgery of the larynx essentially relies on the perfect mastering of every possible diagnostic tool available. As demonstrated by Ruytenberg et al. Magnetic Resonance Imaging has gradually emerged as the preferred method of investigation in such a field due to its superior capability of space and tissue density resolution. By designing dedicated laryngeal radiofrequency coils and applying respiratory-triggered acquisition techniques that minimize breathing and swallowing related artifacts, high-quality images (with an isotropic resolution of up to $1 \mathrm{~mm}$ ) acquired in a few minutes will allow surgeons to obtain a set of nearly histopathologic macro-sections of the entire larynx, with marvelous understanding of the 3D extent of the lesion to be treated.

As already demonstrated by several reports, among which those first published by Blakeslee in 1984 (3) and later reproduced by Peretti et al. (4, 5), Hamzany et al. showed that an accurate pre- and intraoperative diagnostic work-up allows not only to avoid any preemptive biopsy, directly treating a given glottic lesion by a single stage TLM session, but also that it is possible to minimize the rates of over- and undertreatments in terms of depth of partial cordectomy to $10 \%$. In particular, the routine use of intraoperative visual aids such as Narrow Band Imaging, based on the biologic endoscopy concept previously described by two of us (6), may greatly help in reducing the amount 
of surgical mistakes in terms of superficial positive margins, one of the most potent prognosticators in every oncologic surgical procedure, as herein demonstrated in a series of 634 patients by Fiz et al.

Even though the vast majority of papers in the literature details outcomes of primary TLM for management of naive glottic tumors, Meulemans et al. confirm the applicability of such a therapeutic weapon even in the (much more troublesome) scenario of selected post-actinic persistent/recurrent tumors. Even though oncologic outcomes remain satisfactory, not surprisingly the Belgian Authors found a statistically significant lower local recurrence-free survival and ultimate local control rate in such a clinical setting. However, being able to ultimately preserve the larynx in $65 \%$ of their patients, the Authors definitively turned the old tenet upside down that the only possible salvage option after radiation failure would be total laryngectomy.

A comprehensive approach to the categorization of different subgroups of glottic lesions to be treated by TLM is applied by Piazza et al. rigorously assessing their dataset of $410 \mathrm{~T} 1-$ T3 untreated patients and trying to overcome the intrinsic limitations of the contemporary edition of the TNM staging system in giving an affordable prognostication. The resulting 3D map of isoprognostic zones can effectively guide surgeons within their multidisciplinary teams in discussing the pros and cons of transoral management of a glottic tumor, balancing alternative therapeutic options in the search for the best compromise between oncologic cure and functional results.

Even though less frequently encountered and with different loco-regional patterns of spreading, even supraglottic carcinomas may be effectively managed by TLM as herein demonstrated by two independent groups of Italian and German surgeons. In fact, both Carta et al. and Ambrosch et al. report excellent oncologic outcomes in treating T1-T3 (and selected T4 lesions in the German series) with such an approach, claiming optimal vocal and swallowing outcomes that are comparatively better than those traditionally described after open-partial laryngectomies or non-surgical treatments. Interestingly, the Italian group also proposes a slight technical modification to the more advanced type of endoscopic supraglottic resection according to the ELS classification (7), by preserving the inferior third of the arytenoid cartilage and thus greatly reducing the risk of longlasting post-operative aspiration described by others after such a procedure (8). Tumors of the posterior laryngeal compartment, i.e., involving the posterior third of the vocal cords, posterior commissure, and medial aspect of the arytenoid cartilages, have been traditionally regarded as biologically insidious and technically demanding, especially in case of superficial lesions not requiring open surgery, but still located in a difficult area to be managed by the transoral route via a standard orotracheal intubation. Mora et al. herein brilliantly introduce subglottic high frequency jet ventilation through a $4 \mathrm{~mm}$ laser-safe catheter as a method that allows superb control of superficial resection margins in such a rare tumor location.

One of the most limiting factors in teaching and learning TLM techniques is the contemporary relative paucity of anatomical descriptions and text books specifically focusing on structural details from the inside out. This, indeed, is definitively the most intriguing nuance represented by the work of Perotti et al. depicting in great detail the vascular anatomy of the supraglottic, glottic, and subglottic regions, with special attention to practical tips that every transoral surgeon should master in order to anticipate or treat bleeding in such procedures.

Interestingly, about $25 \%$ of papers in the present Research Topic have been devoted to discussion of transoral robotic surgery (TORS) for laryngeal cancer. A comprehensive review on the subject has been performed by Gorphe demonstrating in a balanced way the present pros and cons of such a surgical approach to the laryngeal oncologic issues. Even though comparative analysis between the "classic" TLM technique and TORS are still scant and frequently surpassed by quickly evolving robotic technology, this new approach to minimally-invasive surgery performed through natural orifices is clearly here to stay. For example, some may argue that the reported tracheostomy rate $(32 \%)$ in the large series of supraglottic cancers managed by TORS from Stubbs et al. and, especially, their relatively long decannulation time (4 months) are less appealing than those reported in the major TLM series, two of which are also included in the present collection. However, as usual, direct comparisons between different series, with a different tumor mix treated in diverse health care systems, are difficult and limited in their intrinsic value. Moreover, new robots and improved anesthesiologic techniques may well change the treatment profile of these patients in the near future. The growing active interest in TORS is also evoked by Mäkitie et al. Their short paper is a purely epidemiological view on TORS in the Scandinavian countries, not addressing oncological outcomes, need for additional non-surgical treatment, or complications. The ambition of this paper is no more than an urgent invitation to summarize the need for rigorous data collection within a future clinical trial, as well as for prospectively formulating high management standards to minimize future side effects and undue sequelae. A complicating factor in interpreting the findings is that most of the centers analyzed are obviously in the beginning or the middle of their learning curve. The Authors do not provide scientific data detailing the likely (causal) relationship between the number of surgeries and outcomes, nor on the expected positive effect of their proposed further centralization (9). This criticism prompted some esteemed Editorial Board members to recommend against publication, while others heavily supported its inclusion in the present Research Topic. The final decision, as you can see, was to include such a paper as a photograph of a peculiar moment in the development of TORS in a large portion of the European Community.

In conclusion, we are strongly convinced that some of the nuances presented in this Research Topic, and definitively many others still to be developed or published, will greatly contribute in reshaping the future indications, outcomes, and limits of transoral treatments of laryngeal cancer. Collation of such different hints, suggestions, and proposals starts from the research benches, proceeds through publications and conferences, and ends up in the operatory rooms and outpatient 
clinics of the real world to improve overall laryngeal cancer care. We are deeply honored to be part of such a chain, and truly hope this Research Topic will fuel further interests in readers and scholars.

\section{REFERENCES}

1. Steiner W. Results of curative laser microsurgery of laryngeal carcinomas. Am J Otolaryngol. (1993) 14:116-21. doi: 10.1016/0196-0709(93) 90050-H

2. Remacle M, Eckel HE, Antonelli A, Brasnu D, Chevalier D, Friedrich G, et al. Endoscopic cordectomy. A proposal for a classification by the Working Committee, European Laryngological Society. Eur Arch Otorhinolaryngol. (2000) 257:227-31. doi: 10.1007/s004050050228

3. Blakeslee D, Vaughan CW, Shapshay SM, Simpson GT, Strong MS. Excisional biopsy in the selective management of T1 glottic cancer: a three-year follow-up study. Laryngoscope (1984) 94:488-94. doi: 10.1288/00005537-198404000-00012

4. Peretti G, Cappiello J, Nicolai P, Smussi C, Antonelli AR. Endoscopic laser excisional biopsy for selected glottic carcinomas. Laryngoscope (1994) 104:1276-9. doi: 10.1288/00005537-199410000-00017

5. Peretti G, Piazza C, Berlucchi M, Cappiello J, Giudice M, Nicolai P. Pre- and intraoperative assessment of mid-cord erythroleukoplakias: a prospective study on 52 patients. Eur Arch Otorhinolaryngol. (2003) 260:525-8. doi: 10.1007/s00405-003-0584-0

6. Piazza C, Del Bon F, Peretti G, Nicolai P. "Biologic endoscopy”: optimization of upper aerodigestive tract cancer evaluation. Curr Opin Otolaryngol Head Neck Surg. (2011) 19:67-76. doi: 10.1097/MOO.0b013e328344b3ed

\section{AUTHOR CONTRIBUTIONS}

All authors listed have made a substantial, direct and intellectual contribution to the work, and approved it for publication.

7. Remacle M, Hantzakos A, Eckel H, Evrard AS, Bradley PJ, Chevalier D, et al. Endoscopic supraglottic laryngectomy: a proposal for a classification by the working committee on nomenclature, European Laryngological Society. Eur Arch Otorhinolaryngol. (2009) 266:993-8. doi: 10.1007/s00405-008-0901-8

8. Piazza C, Barbieri D, Del Bon F, Grazioli P, Perotti P, Paderno A, et al. Functional outcomes after different types of transoral supraglottic laryngectomy. Laryngoscope (2016) 126:1131-5. doi: 10.1002/lary.25562

9. Cracchiolo JR, Roman BR, Kutler DI, Kuhel WI, Cohen MA. Adoption of transoral robotic surgery compared with other surgical modalities for treatment of oropharyngeal squamous cell carcinoma. J Surg Oncol. (2016) 114:405-11. doi: $10.1002 /$ jso. 24353

Conflict of Interest Statement: The authors declare that the research was conducted in the absence of any commercial or financial relationships that could be construed as a potential conflict of interest.

Copyright (C) 2018 Piazza, Peretti and Vander Poorten. This is an open-access article distributed under the terms of the Creative Commons Attribution License (CC BY). The use, distribution or reproduction in other forums is permitted, provided the original author(s) and the copyright owner(s) are credited and that the original publication in this journal is cited, in accordance with accepted academic practice. No use, distribution or reproduction is permitted which does not comply with these terms. 


\title{
Vocal Fold Cancer Transoral Laser Microsurgery Following European Laryngological Society Laser Cordectomy Classification
}

\author{
Abie H. Mendelsohn ${ }^{1 *}$ and Marc Joseph Remacle ${ }^{2}$ \\ ${ }^{1}$ Department of Head and Neck Surgery, UCLA David Geffen School of Medicine, Los Angeles, CA, United States, \\ ${ }^{2}$ Department of Otorhinolaryngology Head and Neck Surgery, Centre Hospitalier de Luxembourg - Clinique d'Eich, \\ Luxembourg, Luxembourg
}

\section{OPEN ACCESS}

Edited by:

Cesare Piazza

Istituto Nazionale dei Tumori

(IRCCS), Italy

Reviewed by:

Jeroen Meulemans,

University Hospitals Leuven,

Belgium

Sebastien Vergez, Institut universitaire du Cancer de

Toulouse Oncopole, France

${ }^{*}$ Correspondence:

Abie H. Mendelsohn

AbieMendelsohn@gmail.com

Specialty section: This article was submitted to Head and Neck Cancer, a section of the journal

Frontiers in Oncology

Received: 27 April 2018 Accepted: 05 June 2018

Published: 22 June 2018

Citation:

Mendelsohn AH and Remacle MJ (2018) Vocal Fold Cancer Transoral

Laser Microsurgery Following European Laryngological Society Laser Cordectomy Classification.

Front. Oncol. 8:231.

doi: 10.3389/fonc.2018.00231
Background: The surgical treatment of glottic, or vocal cord, cancer has seen considerable progression over the past several decades. Specifically, there has been a stark transition from open partial laryngectomy surgery to endoscopic laser microsurgical techniques which have been inspired in large part by two landmark studies: Professor Wolfgang Steiner's original case series describing transoral laser microsurgery for glottic cancer (1993) and the European Laryngological Society's (ELS) classification scheme (2000). We performed a comprehensive review of published literature to characterize the pattern of this novel modality as compared with two landmark studies over the past four decades.

Methods: An English literature search was conducted on PubMed for available original investigations on surgical treatment of glottic laryngeal cancer published over the past 40 years. Our Boolean criteria included the following terms: cancer, glottic, laryngeal, surgery, endoscopic, and laser. The publication rates were calculated as annual compound growth as well as corrected growth rates as defined by the Fisher equation for inflation effects.

Results: Our review identified 13,372 studies covering larynx cancer and 3,557 studies covering glottic cancer original studies. Among these, we analyzed the compound annual growth rates and correct growth rates for three distinct publication periods or epochs, prior to 1993, 1993-1999, and 2000-2017. For all but two of the search term groups covering both glottic cancer as well as larynx cancer, there was a substantial growth improvement in the time period following the ELS classification scheme as compared with the growth rate of the time period following Steiner's case series.

Conclusion: The progress toward minimally invasive treatment of glottic cancer has progressed steadily over the past several decades. Analysis of publication show increased growth during the time period following the ELS classification scheme over the time period following Steiner's landmark study. A mistake would be concluding any diminished importance of Professor Steiner's work, instead, our analysis demonstrates the wide-spread adoption of the endoscopic laser cordectomy procedure following the ELS classification system. Complex surgical techniques such as transoral laser microsurgery are optimally disseminated within well-defined classification schemes, though further validation is warranted.

Keywords: laser cordectomy, glottic cancer, voice, endoscopy, KTP 


\section{INTRODUCTION}

Vocal fold cancer, particularly early staged (T1-T2) glottic cancer, is recognized to have reliably excellent cure rates irrespective of treatment modality (1-3). Specifically, local control and laryngectomy-free survival exceeds $90 \%$ following primary radiotherapy or definitive surgical resection. With the oncologic success rates nearly optimized, treatment advances have been motivated to minimize treatment-related morbidity thereby optimizing functional outcomes. External beam radiation advances have been shown with the adoption of intensity modulated radiation therapy (IMRT) which reduced the size and treatment volume. With less of the pharyngolarynx impacted by the effects of radiation, IMRT has been thought to improve voice and swallowing morbidity over conventional radiotherapy designs (4). Similarly, primary surgical strategies have looked to optimize functional outcomes mainly by transitioning oncologic resection from open cervical surgery to transoral endoscopic approaches. Minimizing the area of larynx which is affected by surgery has been shown to optimize airway management as well as post-surgical voicing (5).

The transition from open surgical resection to endoscopic resection has been seen over the past 40 years. As early as the 1970s, Strong and Jako adapted the $\mathrm{CO}_{2}$ laser for endoscopic laryngeal surgery (6). Despite this critical advance in technologic capacity, generally it is Professor Wolfgang Steiner who was attributed with the popularization of the $\mathrm{CO}_{2}$ laser endoscopic resection of glottic cancers. In 1993, Steiner published the seminal work describing his considerable experience and results for endoscopic $\mathrm{CO}_{2}$ laser resection of glottic cancers (7). Steiner's approach was revolutionary, bucking the dogma of oncology surgeons of the time who demanded en-bloc tumor resections. Instead, Steiner described a stepwise process toward piecemeal removal of the glottic tumors. As Steiner argued, a surgeon could use the $\mathrm{CO}_{2}$ laser to bisect the tumor, thereby establishing the deep oncologic margin, and subsequently resect the tumor areas anterior and posterior to this bisecting cut. In this seminal manuscript, Steiner reported excellent and reliable oncologic outcomes with preservation of laryngeal function. However, anecdotally, many surgeons world-wide argued against Steiner's approach on the basis of inability to reproduce the reported success rates with the technique.

Following Steiner's popularization of the approach, the endoscopic $\mathrm{CO}_{2}$ laser surgery for glottic cancers continued to progress slowly until a landmark manuscript by the collaborative work of the European Laryngological Society (ELS), comprised of the leading European authorities in laryngeal cancer surgery. The statement manuscript called for a unified classification scheme for endoscopic $\mathrm{CO}_{2}$ laser resection of glottic cancer, otherwise termed $\mathrm{CO}_{2}$ laser cordectomy. One of the primary goals of the ELS consensus statement was to create a uniform language for outcomes comparison across intuitions and manuscripts. Another critical goal of the ELS system was to define a reliably reproducible systematic approach toward endoscopic resection which would be defined by the invasiveness and areas involved with tumor growth $(8,9)$. Therefore, with the establishment of the ELS cordectomy classifications much of the judgment calls and reliance on clinical experience needed with Steiner's approach was improved. The ELS laser cordectomy system proposed a sequential protocol that surgeons could utilize based on their assessment of the extent of cancer. Specifically, bulky unilateral tumors might be treated with an intramuscular cordectomy (ELS type III), whereas deeply invasive tumors might be better addressed via a complete cordectomy (ELS type IV).

As such, it has been suggested that the ELS classification system succeeded in not only offering a common language to compare surgical results but more importantly offering specific surgical specifications for the spectrum of glottic invasive carcinoma. But this suggestion has yet to be evaluated or confirmed. Some investigators have studied the evolving treatment patterns of laryngeal cancer through national database analyses, yet the use of central data-banks hold significant limitations and assumptions which limit their ability to answer our question. Therefore, we sought to analyze the impact of the ELS Cordectomy classification on the evolution of endoscopic laser resection of glottic cancer by analyzing peer-reviewed medical publication rates.

\section{MATERIALS AND METHODS}

The historical literature citations of the two landmark studies of interest were analyzed. To perform this analysis, the manuscripts were searched within the Web of Science Core Collection Database which provides comprehensive citation count and analysis. (http://apps.webofknowledge.com/WOS_GeneralSearch_input. do? product $=$ WOS\&search $\_$mode $=$GeneralSearch $\& S I D=7 \mathrm{Ai}$ wMkTvq28ge7aOYLA\&preferencesSaved=, accessed May 27, 2018) However, following this analysis it was determined that no conclusion could be made based on the comparative impact on the field of glottic cancer.

We, therefore, sought to identify the rate of publications regarding the topic of endoscopic resection of glottic cancer. A literature search was conducted on PubMed for available English Language publications on the subject of interest. All articles were recorded initially, but were then limited based on the publication year of 1975. This limit was chosen to offer comparative publication total for comparisons across all search term groups, as several search groups totaled zero prior to 1975 . The search was performed in January 2018, therefore, publication year of 2017 was included while publication year of 2018, having only just started, was excluded. Therefore, all of our search term groups were limited between 1975 and 2017.

The search term groups were performed with two base search titles. The first round of search groups was done using the titles of both "GLOTTIC" and "CANCER." The second round of search groups was done using the titles of both "LARYNX" and "CANCER." Both search titles were then redefined by more specific Boolean criteria which added to the base titles the following terms: surgery, then including endoscopic and/or Laser (all performed individually as opposed to a single Boolean search term). Once the search was completed, we computed the annual manuscript volumes. Annual manuscript volumes were then converted into cumulative growth totals through standard Excel (Volume 15.32, Microsoft, Seattle, WA, USA) formulation.

Our goal was to compare the effects that the two major papers in the field of endoscopic laser treatment for glottic cancer on 
publication rates. Therefore, our searches were divided into three distinct time periods, or eras. The first period was defined as "before Dr. Steiner's landmark publication" (7), or 1980-1992 (with cumulative total for beginning total $=$ publications from 1975-1980). Notably, though the literature search included manuscripts from 1975 and on, the first group was defined as from 1980 and on in order that the baseline growth rate would be representative of the manuscripts published in the 5 years prior to the group start date. Starting the analysis with a previous annual total of zero creates an extremely skewed measure of publication growth. The second period was defined as "after Dr. Steiner's publication but before the ELS cordectomy classification," or 1993-1999. The third period was defined as "after ELS classification proposal," (8) or 2000-2017.

We then took the previously calculated cumulative totals and then converted these into a compound annual growth rate (CAGR). The formula used for CAGR is well described in the economics field:

$\mathrm{CAGR}=(\text { Ending Value } / \text { Beginning Value })^{(1 / \text { number of years })}-1$.

By converting the cumulative totals into the CAGR, we would adjust for the varying overall number of years in each of the three defined time periods.

However, a comparison between the time period growth rates at this point offers inaccurate comparison as the overall change in medical journal scholarship and publications which is expected when looking over a long time period such as this. Therefore, a correction was required which could adjust for this overall publication growth rate. If we are to accept that there is regular increase in medical publications over the years, we may describe this overall growth rate as publication "inflation." To address the effect of publication inflation, we return to the field of economics. The Fisher equation in financial mathematics and economics estimates the relationship between nominal and real interest rates under inflation. Which, after derivations holds true to the initial equation of $(r \approx i-\pi)$, where $r=$ real growth rate, $i=$ nominal growth rate, $\pi=$ inflation rate.

Therefore, as our primary study goal is the analysis of the growth of publications describing endoscopic laser surgery for glottic (or laryngeal) cancer, we can define the overall growth rate of manuscripts within the field as manuscripts related to the title search terms of "glottic (or laryngeal) cancer," without mention of surgery or laser. Therefore, we can define the growth rate of manuscripts published under the search terms glottic (or laryngeal) and cancer as a so-called inflation rate $(\pi)$. We can then use the growth rate of the specific terms of endoscopic and/or laser combined with the baseline terms of glottic (or laryngeal) and cancer as the rate we measure as the nominal growth rate $(i)$. Thus, to determine the real growth rate $(r)$ of manuscript publications within the term of interest we will subtract the inflation rate from the nominal growth rate $(r \approx i-\pi)$.

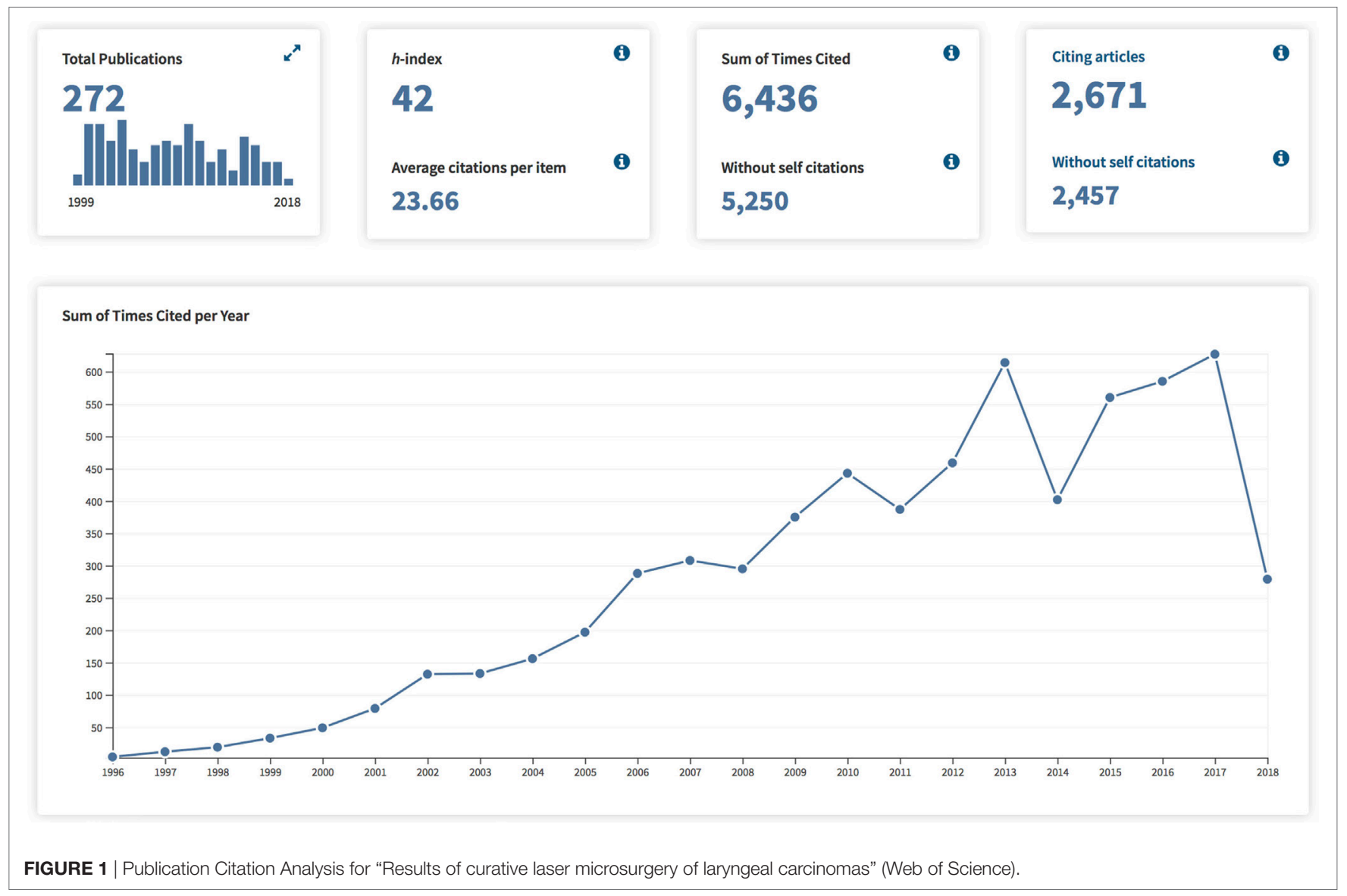




\section{RESULTS}

We sought to evaluate the effects on the medical scholarship of the two landmark studies which have help shape the field of transoral laser microsurgery for glottic cancer. We first compared the growth rates to the Web of Science citation trends as displayed in Figures 1 and 2. Both of the publications of interest in this analysis demonstrate continuously increasing citations throughout the years following initial publication. The study by Steiner demonstrates over double the total number of citing articles (2,671 as compared with 1,177 for the ELS classification scheme), as well as a substantially increased Sum of Times Cited (6,436 as compared with 2,974 for the ELS classification scheme). However, what we see is that based on the aggregate time line, the vast majority of Times Cited occur within the most recent 7 years which is the interval time span between the two publications. Therefore, additional analysis was required to evaluate the publication effects of the two articles of interest.

We, therefore, proceeded to evaluate the publication growth rate within three distinct time periods as related to the publication years of the landmark manuscripts defining the $\mathrm{CO}_{2}$ laser endoscopic resection of early glottic cancer. Cumulative totals for English Language publications for "GLOTTIC and CANCER" between 1975 and 2017 was 3,557. Cumulative totals for English Language publications for "LARYNX and CANCER" between 1975 and 2000 was 13,372. Tables 1 and 2 delineate both the CAGR of the publications as well the corrected annual growth rates using the title search term of glottic as well as larynx.

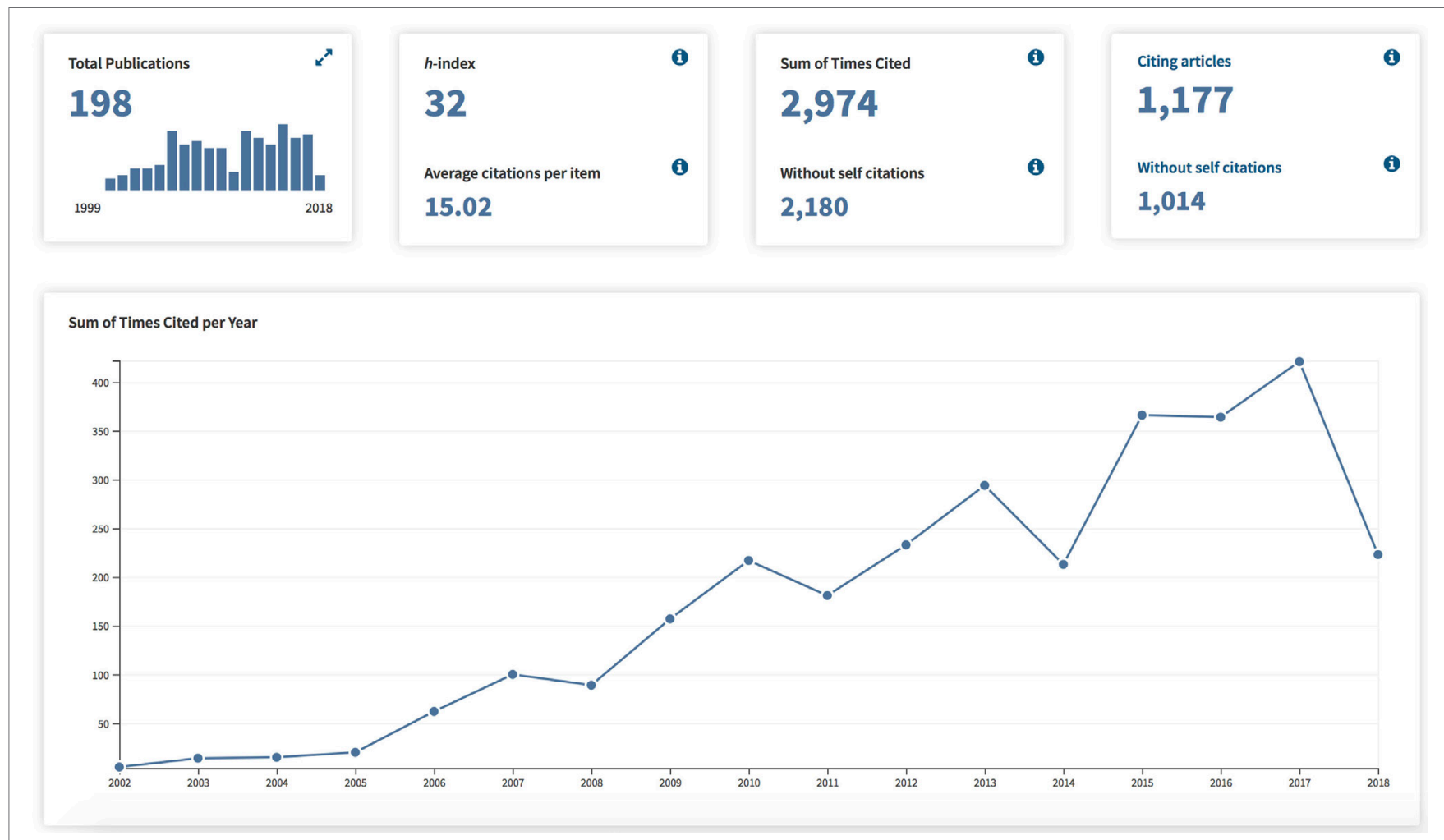

FIGURE 2 | Publication Citation Analysis for "Endoscopic cordectomy. A proposal for a classification by the Working Committee, European Laryngological Society" (Web of Science).

TABLE 1 | Publication rates for glottic cancer.

\begin{tabular}{|c|c|c|c|c|c|c|}
\hline & \multicolumn{3}{|c|}{$\begin{array}{l}\text { Compound annual publication growth } \\
\text { rate }\end{array}$} & \multicolumn{3}{|c|}{$\begin{array}{c}\text { Real (or corrected) annual publication growth } \\
\text { rate }\end{array}$} \\
\hline & 1980-1992 & 1993-1999 & 2000-2017 & $1980-1992 \mathrm{c}$ & 1993-1999c & 2000-2017c \\
\hline Glottic and cancer and surgery & $9.51 \%$ & $6.54 \%$ & $4.96 \%$ & $0.14 \%$ & $-0.06 \%$ & $0.52 \%$ \\
\hline Glottic and cancer and surgery and laser & $20.57 \%$ & $8.30 \%$ & $6.85 \%$ & $11.20 \%$ & $1.70 \%$ & $2.40 \%$ \\
\hline Glottic and cancer and surgery and endoscopic & $11.75 \%$ & $9.48 \%$ & $7.13 \%$ & $2.38 \%$ & $2.87 \%$ & $2.69 \%$ \\
\hline
\end{tabular}

The compound annual growth rate is calculated for three distinct time periods: prior to Steiner's landmark publication (1980-1992), after Steiner's landmark publication until European Laryngological Society (ELS) Cordectomy Classification (1993-1999), and after the ELS Cordectomy Classification (2000-2017). Also shown are the corrected growth rates by adjusting the surgical publication rates based on overall growth rates within the topic of glottic cancer. 
TABLE 2 | Publication rates for larynx cancer.

\begin{tabular}{|c|c|c|c|c|c|c|}
\hline & \multicolumn{3}{|c|}{$\begin{array}{l}\text { Compound annual publication growth } \\
\text { rate }\end{array}$} & \multicolumn{3}{|c|}{$\begin{array}{l}\text { Real (or corrected) annual publication growth } \\
\text { rate }\end{array}$} \\
\hline & 1980-1992 & 1993-1999 & 2000-2017 & 1980-1992c & 1993-1999c & 2000-2017c \\
\hline Larynx and cancer & $11.27 \%$ & $6.52 \%$ & $4.44 \%$ & - & - & - \\
\hline Larynx and cancer and surgery & $10.49 \%$ & $6.86 \%$ & $5.46 \%$ & $-0.78 \%$ & $0.34 \%$ & $0.92 \%$ \\
\hline Larynx and cancer and surgery and laser & $17.03 \%$ & $7.43 \%$ & $5.97 \%$ & $5.76 \%$ & $0.91 \%$ & $1.42 \%$ \\
\hline Larynx and cancer and surgery and endoscopic & $11.96 \%$ & $7.95 \%$ & $7.34 \%$ & $0.70 \%$ & $1.43 \%$ & $2.80 \%$ \\
\hline Larynx and cancer and surgery and endoscopic and laser & $22.23 \%$ & $7.22 \%$ & $6.60 \%$ & $10.97 \%$ & $0.69 \%$ & $2.05 \%$ \\
\hline
\end{tabular}

The compound annual growth rate is calculated for three distinct time periods: prior to Steiner's landmark publication (1980-1992), after Steiner's landmark publication until European Laryngological Society (ELS) Cordectomy Classification (1993-1999), and after the ELS Cordectomy Classification (2000-2017). Also shown are the corrected growth rates by adjusting the surgical publication rates based on overall growth rates within the topic of larynx cancer.

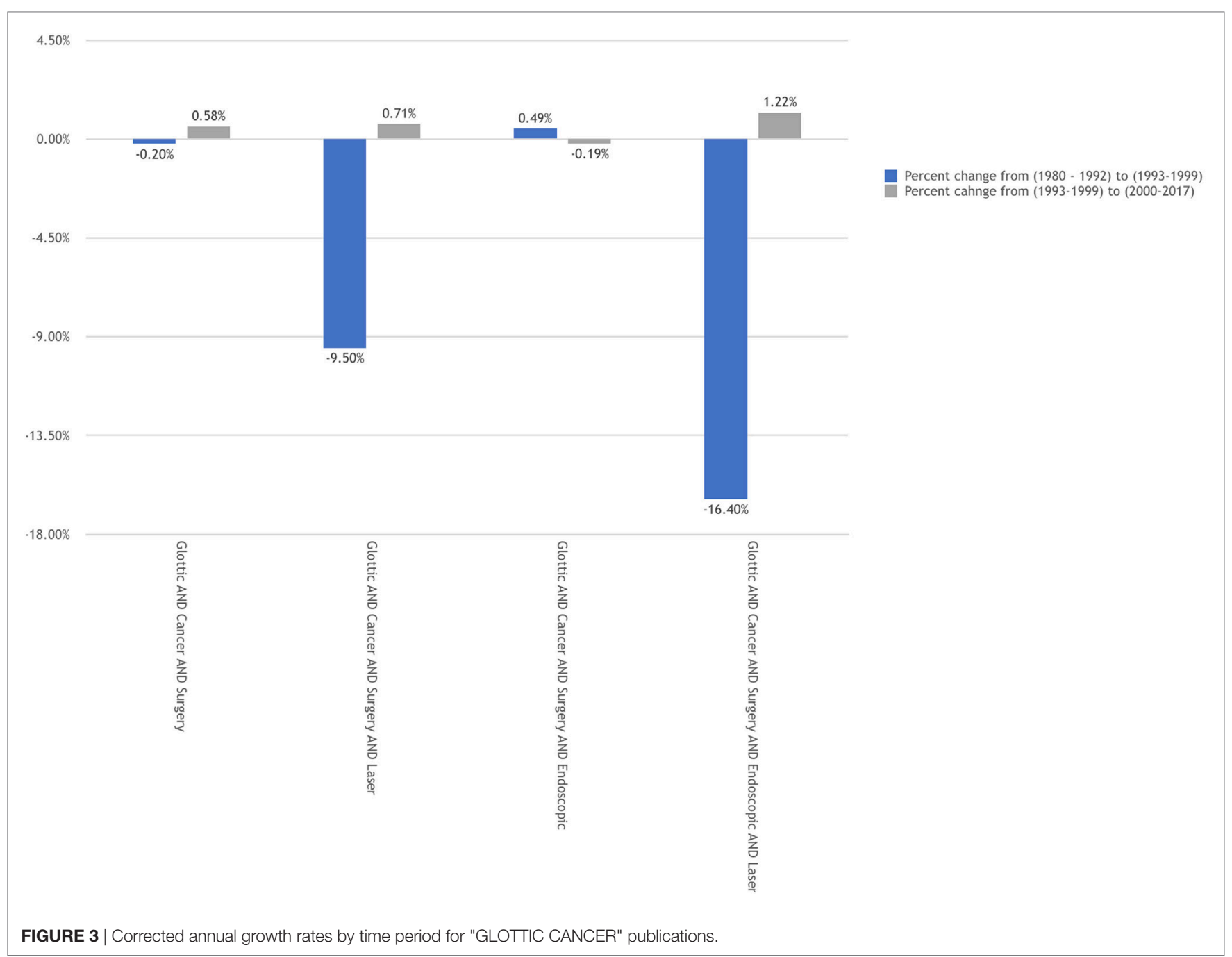

Generally, the time period before 1993 saw both large annual publication growth rates for glottic terms as well as larynx terms. Though the uncorrected annual growth rates for the periods of 1993-2000 and 2000-2017 showed consistent publication increases, when evaluating the growth rate specifically for the surgical management of glottic/larynx cancer the corrected values diminish significantly.
The goal of the analysis was to evaluate the effects of the two landmark manuscripts on the publication rates. To assess this effect, the change (delta) in the corrected annual publication growth rates are displayed in Figures 3 and 4. When comparing the two time points (1993 versus 2000) we see that three of the four search groups for each search title maintained a larger annual growth rate in the period following the ELS classification 


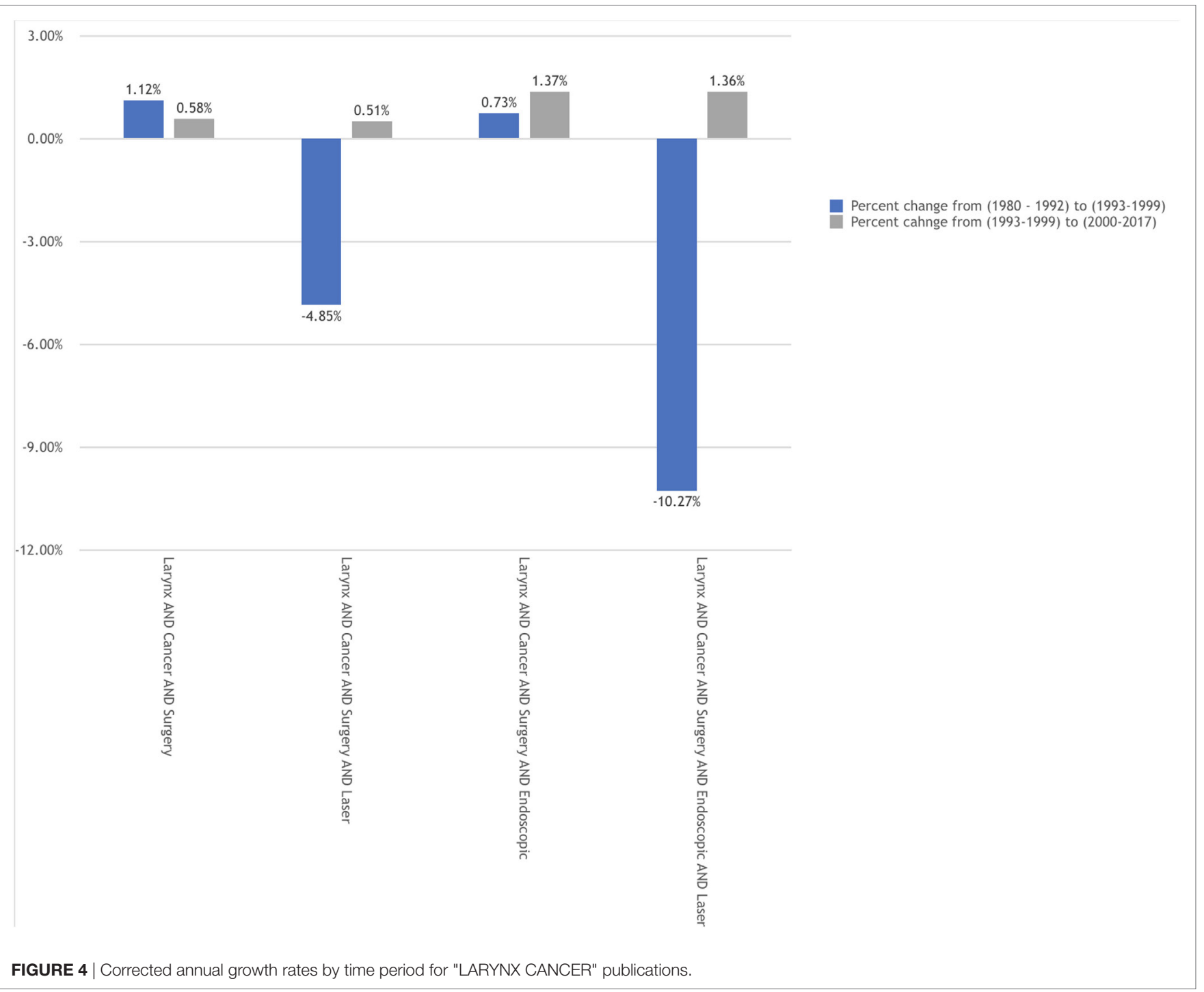

scheme. Interestingly, several of the search groups demonstrated a negative change in the annual growth rate comparing prior to versus following 1993. This negative growth rate change was only seen in one search group and at a very slight negative rate $(-0.19 \%)$ for that single group.

\section{DISCUSSION}

We sought to evaluate the effect of two landmark publications in the field of surgery for glottic cancer. Publication rates were chosen as the effect of interest in this study. However, a sideby-side comparison of publication rates between the time periods of interest results in skewed analysis in that publication rates for glottic cancer overall is not stable during these time periods. As overall medical journal publication rates can vary substantially, as well as publications covering the entire topic of throat cancer, the smaller subset of publications regarding the surgical care within our area of concern would be likewise affected. As such, the present analysis was adjusted to correct the overall change in publications over these time periods for the general area of interest, both glottic cancer as well as larynx cancer.

Utilizing a correct CAGR, we first calculated the growth rate of publications within the topics of glottic and larynx cancer covering endoscopic/laser/surgery prior to Professor Wolfgang Steiner's landmark study describing his experience. We then calculated the corrected rates between Steiner's work and publication of the ELS classification scheme of endoscopic laser cordectomy. Finally, we calculated the correct annual publication growth rate for the time period following the ELS paper. We demonstrated a substantial increase in the publications on endoscopic/laser/ surgery as compared with the time periods prior.

An erroneous conclusion would be to create a direct comparison between the importance of these two landmark studies, somehow considering one manuscript superior than another. In point of fact, a comparison of importance would be a wasteful exercise without value. Instead, what this analysis set out to examine is the overall dispersion and propagation effects of the studies of interest. Stated in the form of a question, which work 
offered a wide acceptance rate of endoscopic laser surgery for glottic cancer? The value of such a question is to investigate the influencing factors of advancing surgical techniques to provide the guidance for the upcoming future surgical advances.

Steiner's publication describing a technique so aggressively bucking the accepted oncologic dogma that its resulting aftershocks were felt for years following. Though initially met with heavy skepticism and outright rejection, Steiner's undeniable success combined with his vast patient experience ultimately provided the foundation for the technique as it exists today. However, as demonstrated in the current analysis, glottic cancer patients outside of Göttingen were unlikely to reliably have this surgical option available to them. We may only theorize the reasons as why Steiner's proven successful techniques were not being practiced and published with regularity. Some would suggest that their success rates were not readily replicated. Others would suggest that the anatomic and surgical complexity in which the piecemeal resections required left few surgeons with the confidence to promote the approach.

In fact, the variability of the transoral laser microsurgery techniques being described in the 1980s and 1990s was a major motivating factor for the ELS to formalize their recommendations. In quoting from their original manuscript, "We believe that non-standardized surgery, which requires years of training to understand its limits, offers little reproducibility to the majority of laryngologists." Herein, we propose that the analysis gives credence to the ELS original goals of creating a technique and treatment option which offers wide application.

When reviewing the literature on the topic we have seen several authors to investigate the current practice trends for the treatment of glottic cancers utilizing one of several methods. First would be to query national patient databases by diagnosis $(10,11)$. However, while such large-scale databases offer substantial sample size analysis, the dependence on surgical coding leaves very much to be assumed regarding actual surgical techniques utilized. A second approach has been surgeon survey studies $(12,13)$. Even in the published studies which boast a high level of surgeon participation and response, these studies suffer from dependence on memory bias and assumption.

\section{REFERENCES}

1. Huang G, Luo M, Zhang J, Liu H. Laser surgery versus radiotherapy for T1a glottic carcinoma: a meta-analysis of oncologic outcomes. Acta Otolaryngol (2017) 137(11):1204-9. doi:10.1080/00016489.2017.1353706

2. Abdurehim Y, Hua Z, Yasin Y, Xukurhan A, Imam I, Yuqin F. Transorallaser surgery versus radiotherapy: systematic review and meta-analysis for treatment options of T1a glottic cancer. Head Neck (2012) 34(1):23-33. doi:10.1002/hed.21686

3. Higgins KM. What treatment for early-stage glottic carcinoma among adult patients: $\mathrm{CO}_{2}$ endolaryngeal laser excision versus standard fractionated external beam radiation is superior in terms of cost utility? Laryngoscope (2011) 121(1):116-34. doi:10.1002/lary.21226

4. Rosenthal DI, Fuller CD, Barker JL Jr, Mason B, Garcia JA, Lewin JS, et al. Simple carotid-sparing intensity-modulated radiotherapy technique and preliminary experience for T1-2 glottic cancer. Int J Radiat Oncol Biol Phys (2010) 77(2):455-61. doi:10.1016/j.ijrobp.2009.04.061

5. Motta G, Esposito E, Motta S, Tartaro G, Testa D. $\mathrm{CO}_{(2)}$ laser surgery in the treatment of glottic cancer. Head Neck (2005) 27(7):566-73; discussion 573-4. doi:10.1002/hed.20135
The present analysis here too relies on assumptions, mainly that all the publications included in the calculations are actually relevant to the topic instead of artifacts of search algorithms. This concern was deemed manageable in the authors' opinion as the publication numbers, and by extension the publication growth rates, were compared from group to group and, therefore, any artifacts presents would be canceled out during the comparative calculations. The second assumption was that publication rates in and of themselves stand as a proxy for the adoption of endoscopic laser surgery for glottic cancer. Admittedly this consideration requires a jump when considering effect and causation; however, in the authors' opinion this is a relatively small jump especially when considering the limitations of the alternative investigative strategies. There are several possible alternative explanations of the results of the data analysis including increases due to an undetermined long learning curve for the techniques with subsequent underreporting of results in the early years as well as increased attention for the technique during otolaryngology conferences.

\section{CONCLUSION}

After comprehensive review regarding the endoscopic laser surgical treatment of glottic cancer, we have demonstrated that scientific publications increased in frequency following the European Laryngological Society (ELS) Cordectomy Classification proposal. This increase was beyond the changes seen in publication seen over a decade earlier following Professor Steiner's landmark paper on the laser resection of glottic cancer. We conclude that the standardization of advanced surgical techniques as seen with the ELS classification scheme improves widespread adoption leading to increased patient access.

\section{AUTHOR CONTRIBUTIONS}

AM was involved in research planning, data acquisition, and manuscript organization. MR was involved in research planning, supervision, manuscript revision, and oversight.

6. Strong MS, Jako GJ. Laser surgery in the larynx. Early clinical experience with continuous $\mathrm{CO}_{2}$ laser. Ann Otol Rhinol Laryngol (1972) 81(6):791-8. doi:10.1177/000348947208100606

7. Steiner W. Results of curative laser microsurgery of laryngeal carcinomas. Am J Otolaryngol (1993) 14(2):116-21. doi:10.1016/0196-0709(93) 90050-H

8. Remacle M, Eckel HE, Antonelli A, Brasnu D, Chevalier D, Friedrich G, et al. Endoscopic cordectomy. A proposal for a classification by the Working Committee, European Laryngological Society. Eur Arch Otorhinolaryngol (2000) 257(4):227-31. doi:10.1007/s004050050228

9. Remacle M, Van Haverbeke C, Eckel H, Bradley P, Chevalier D, Djukic V, et al. Proposal for revision of the European Laryngological Society classification of endoscopic cordectomies. Eur Arch Otorhinolaryngol (2007) 264(5):499-504. doi:10.1007/s00405-007-0304-2

10. Stokes WA, Abbott D, Phan A, Raben D, Lanning RM, Karam SD Patterns of care for patients with early-stage glottic cancer undergoing definitive radiation therapy: a national cancer database analysis. Int J Radiat Oncol Biol Phys (2017) 98(5):1014-21. doi:10.1016/j.ijrobp.2017. 03.050 
11. Mourad M, Dezube A, Moshier E, Shin E. Geographic trends in management of early-stage laryngeal cancer. Laryngoscope (2016) 126(4):880-4. doi:10.1002/lary.25768

12. Makki FM, Williams B, Rajaraman M, Hart RD, Trites J, Brown T, et al. Current practice patterns in the management of glottic cancer in Canada: results of a national survey. JOtolaryngol Head Neck Surg (2011) 40(3): 205-10.

13. DiNardo LJ, Kaylie DM, Isaacson J. Current treatment practices for early laryngeal carcinoma. Otolaryngol Head Neck Surg (1999) 120(1):30-7. doi:10.1016/S0194-5998(99)70366-9
Conflict of Interest Statement: The authors declare that the research was conducted in the absence of any commercial or financial relationships that could be construed as a potential conflict of interest.

Copyright $\odot 2018$ Mendelsohn and Remacle. This is an open-access article distributed under the terms of the Creative Commons Attribution License (CC BY). The use, distribution or reproduction in other forums is permitted, provided the original author(s) and the copyright owner are credited and that the original publication in this journal is cited, in accordance with accepted academic practice. No use, distribution or reproduction is permitted which does not comply with these terms. 
OPEN ACCESS

Edited by: Cesare Piazza, Istituto Nazionale dei Tumori (IRCCS), Italy

Reviewed by: Marco Ravanelli, Università di Brescia, Italy

Sandro J. Stoeckli, Kantonsspital St. Gallen,

Switzerland

${ }^{*}$ Correspondence: Thomas Ruytenberg t.ruytenberg@/umc.nl

Specialty section: This article was submitted to Head and Neck Cancer, a section of the journal

Frontiers in Oncology

Received: 11 April 2018 Accepted: 25 May 2018 Published: 06 June 2018

Citation: Ruytenberg T, Verbist BM, Vonk-Van Oosten J, Astreinidou E, Sjögren EV and Webb AG (2018) Improvements in High Resolution Laryngeal Magnetic Resonance Imaging for Preoperative Transoral Laser Microsurgery and Radiotherapy Considerations in Early Lesions.

Front. Oncol. 8:216 doi: 10.3389/fonc.2018.00216

\section{Improvements in High Resolution Laryngeal Magnetic Resonance Imaging for Preoperative Transoral Laser Microsurgery and Radiotherapy Considerations in Early Lesions}

Thomas Ruytenberg ${ }^{1 *}$, Berit M. Verbist ${ }^{2}$, Jordi Vonk-Van Oosten ${ }^{2}$, Eleftheria Astreinidou ${ }^{3}$, Elisabeth V. Sjögren ${ }^{4}$ and Andrew G. Webb ${ }^{1}$

'C.J. Gorter Center for High Field MRI, Department of Radiology, Leiden University Medical Center, Leiden, Netherlands, ${ }^{2}$ Department of Radiology, Leiden University Medical Center, Leiden, Netherlands, ${ }^{3}$ Department of Radiotherapy, Leiden University Medical Center, Leiden, Netherlands, ${ }^{4}$ Department of ENT - Head and Neck Surgery, Leiden University Medical Center, Leiden, Netherlands

As the benefits, limitations, and contraindications of transoral laser microsurgery (TLM) in glottic carcinoma treatments become better defined, pretreatment imaging has become more important to assess the case-specific suitability of TLM and to predict functional outcomes both for treatment consideration and patient counseling. Magnetic resonance imaging (MRI) is the preferred modality to image such laryngeal tumors, even though imaging the larynx using MRI can be difficult. The first challenge is that there are no commercial radiofrequency (RF) coils that are specifically designed for imaging the larynx, and performance in terms of coverage and signal-to-noise ratio is compromised using general-purpose RF coils. Second, motion in the neck region induced by breathing, swallowing, and vessel pulsation can induce severe image artifacts, sometimes rendering the images unusable. In this paper, we design a dedicated RF coil array, which allows high quality high-resolution imaging of the larynx. In addition, we show that introducing respiratory-triggered acquisition improves the diagnostic quality of the images by minimizing breathing and swallowing artifacts. Together, these developments enable robust, essentially artifact-free images of the full larynx with an isotropic resolution of $1 \mathrm{~mm}$ to be acquired within a few minutes.

Keywords: glottic carcinoma, transoral laser microsurgery, magnetic resonance imaging, laryngeal imaging, radiofrequency coil arrays, imaging protocols

\section{INTRODUCTION}

Early as well as moderately advanced laryngeal cancers are both highly treatable conditions, with the main treatment options consisting of transoral laser microsurgery (TLM), open partial laryngectomy (OPL), and radiotherapy (RT). All three modalities are used both in primary as well as recurrent disease, and treatment choice depends on the extent of the lesion as well as local therapeutic protocols and patient-specific factors such as age, comorbidity, and patient preference. 
Advances in therapy over the past two decades have enabled the relative benefits and limitations of the various treatment methods to be better understood. In particular, the indications for TLM have been more clearly defined (1).

The benefits of TLM include the low morbidity and short treatment time associated with endoscopic removal of the tumor. Functional outcomes are better than after OPL (2) and, for early lesions, they are comparable to RT (3). In moderately advanced lesions, there is as of yet a lack of comparative evidence between TLM and RT concerning functional outcome to draw any definite conclusions $(4,5)$. Finally, as TLM leaves all treatment options open after treatment, larynx preservation is generally higher than after RT (5-7). However, as TLM has evolved, and moderately advanced tumors are increasingly being treated, tumor extension to certain subsites within the larynx have been associated with a higher risk of recurrence after TLM (1). This has put an increasing requirement on high-quality radiological imaging to select those cases most suited for a transoral approach as opposed to open surgery or RT.

Imaging has become crucial for accurate evaluation, not only of tumor localization and size, but also for the involvement of subsites for which the suitability of TLM is still under debate, such as the pre- and posterior paraglottic space, the cricothyroid membrane, and the inner lamina of the thyroid cartilage (1). Magnetic resonance imaging (MRI) is increasingly used to distinguish between tumor and edema or fibrosis (8). In addition, in tumors in which surgery is indicated, imaging can help delineate tumor borders and predict the extent of the resection needed. This information can then be used in preoperative patient counseling regarding functional results, which are related to the extent of the resection. Finally, as the role of TLM expands, high quality imaging has become increasingly important in postoperative follow-up for early detection of submucosal recurrence, therefore, maintaining the possibility to perform open partial salvage surgery (1) or administering salvage RT. As a result, as treatment selection, patient counseling and follow-up monitoring for primary and recurrent early and moderately advanced laryngeal cancer evolves, the demand for high quality, robust, high resolution imaging has increased accordingly.

In tumors where RT is chosen as the preferred treatment of a glottic cancer, high-resolution imaging plays an equally important role. The goal of RT is to deliver a high dose to the tumor and minimize the dose to the surrounding healthy tissues in order to reduce vocal dysfunction, dysphagia, arytenoid edema, and carotid artery injuries (9). This goal can only be achieved if the tumor is defined with high accuracy, i.e., when high resolution imaging is available.

Historically, computed tomography (CT) has been the imaging modality of choice, particularly for RT (10). CT is a robust modality due to very short acquisition times and high signal-to-noise ratio (SNR), but is limited in its utility since differentiation between healthy tissue and tumors can be difficult $(10,11)$. Imaging the larynx using MRI became a useful alternative to CT in the late 1980 s and early 1990s primarily due to the development of local surface coils, which enabled higher resolution imaging over a field-of-view, which could be localized to the larynx (12-15). The lack of ionizing radiation, greater inter-tissue contrast, and multiplanar imaging capabilities were all advantages of MRI over CT, but long acquisition times limited the spatial resolution that could be achieved. Only when parallel imaging became available to reduce MRI acquisition times was it possible to achieve high spatial resolution in a realistic scanning time (16). However, laryngeal imaging is still problematic. Current MRI protocols in early glottis carcinoma primarily use general purpose commercial local surface coils (which can also be combined with a head coil), or phased array head/neck coils $(8,17-23)$. As these coils (for example two loops as shown in Figure 1A) are not specifically designed for laryngeal imaging, correct positioning centered on the vocal cords is critical, and needs to be done carefully and precisely by an experienced technician. Problems also arise with different-sized necks, which require a different physical separation of the coils. If the coils are placed too close together then they couple to one another, reducing the signal-to-noise, and also result in too small a field-of-view, a reduced penetration depth, and poor parallel imaging performance (including the possibility of fold-back artifacts): a typical example is shown in Figure 1B. If the loops are placed too far apart, or the patient has a large neck, signal voids within the larynx may occur, as shown in Figure 1C. In addition, the separate loops are prone to displacement during the examination, which increases the patient handling time and may induce patient discomfort.

Even if proper positioning of the coil has been performed, reconstructed images of the larynx often suffer from patientrelated artifacts, which in some cases render images non-useful for diagnosis. One common cause is movement of the larynx during data acquisition, as shown in Figure 1D. Movement is unavoidable during the scanning protocol, as breathing causes the vocal cords to move. Swallowing, which moves the full larynx up to several centimeters in the feet-head direction, may occur

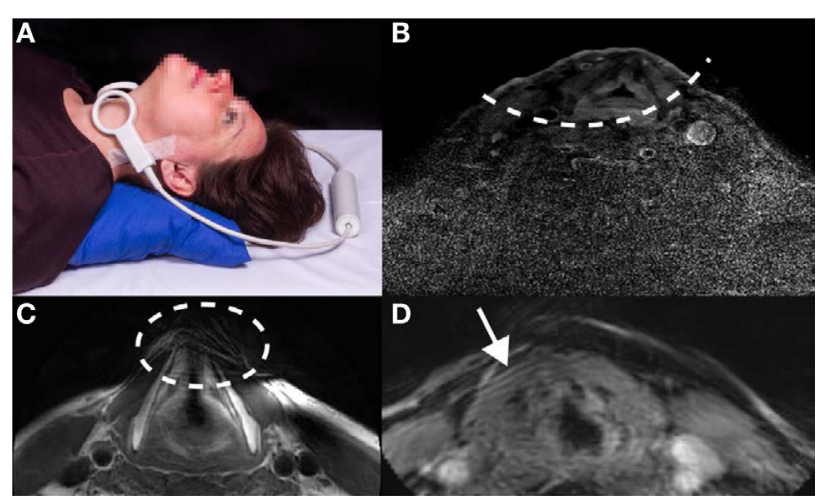

FIGURE 1 | Illustrations of the challenges associated with current approaches to laryngeal magnetic resonance imaging. (A) Two individual, commercial circular receive coils, each with a diameter of $4 \mathrm{~cm}$, are placed around the larynx. Positioning in this particular setup is critical for imaging the larynx and can be experienced as uncomfortable by the subject since the loops have to be held firmly in place with tape and/or velcro straps. Written informed consent was obtained from the subject for publication of this photo. (B) Images acquired with a limited penetration depth due to incorrect coil positioning, (C) images showing signal voids in the anterior part of the larynx due to incorrect coil positioning, and (D) images showing movement artifacts due to subject breathing. 
and can produce severe image artifacts. In addition, pulsation in the blood vessels in the neck commonly creates flow artifacts, which can extend into the larynx region, depending on the imaging protocol used.

In this paper, we report on our work to improve the robustness and performance of high resolution laryngeal MRI. This consisted of two different aspects: first the design, construction, and testing of a flexible receive-array coil, which can adapt to different neck sizes, be worn comfortably by the patient, and enable robust parallel imaging for reduced scanning time. In addition, the array can be used for patients who have to be scanned in a RT fixation mask and it is, to our knowledge, the first time that a coil has been specifically designed to fit such fixation devices. In parallel, we investigated the use of different scan protocols to reduce image artifacts associated with patient motion and pulsatile blood flow.

\section{MATERIALS AND METHODS}

\section{Coil Development}

The dedicated radiofrequency $(\mathrm{RF})$ coil receive array was designed to be flexible to accommodate different neck sizes and to have a high degree of isolation between individual elements to allow high acceleration factors while maintaining a high $\operatorname{SNR}(16,24)$. The number of coils in the receive array represents a compromise: a large number enables a high degree of acceleration, but increases the complexity of the networks required to decouple each of the coils and reduces the flexibility of the overall structure. In order to maintain a high degree of flexibility, as well as maintaining a high degree of decoupling under the different flexation angles required by different-sized necks, we constructed a one-dimensional four element array, as shown in Figure 2.

The dedicated coil is a four-element receive-only array and consists of four identical loops ( $60 \mathrm{~mm} \times 60 \mathrm{~mm}$ each). Inter-element decoupling is achieved using a method termed induced current elimination (25) in combination with preamplifier decoupling, which uses a low impedance pre-amplifier acting as a current transformer (24). This combination results in a coupling of less than $0.1 \%$ for neighboring elements and $1 \%$ for non-neighboring elements. This high level of decoupling is necessary to enable efficient and artifact-free parallel imaging performance (16). Each coil is tuned at $128 \mathrm{MHz}(3 \mathrm{~T})$ and impedance matched to $50 \Omega$ using a lattice balun, which also suppresses common mode currents. Further common mode suppression was achieved by using floating cable traps (26). Decoupling from the transmit body coil (detuning) is performed by a single active PIN diode trap in the each of the resonant loops. A fuse ( $250 \mathrm{~mA}$ very fast acting fuse; Littlefuse $0251.250 \mathrm{MXL}$ ) is integrated into every loop to provide intrinsic safety in the case of any failure in detuning by disabling the coil in case of abnormal high currents.

The printed circuit board of the coil is etched on $0.125 \mathrm{~mm}$ thick FR4, which is flexible enough to be mounted on a curved $1 \mathrm{~mm}$ thick acrylic sheet to provide robustness to the coil as well as flexibility. The geometry of the coil is chosen to fit closely but comfortably around the neck and due to the flexibility of the coil, it easily accommodates different neck sizes. This also opens the possibility for the coil to fit on a RT mask, used to secure the head and neck during RT treatment.

\section{Protocol Development}

Magnetic resonance imaging scans were performed using a 3 T Philips Ingenia system, with the scan parameters shown in Table 1. In vivo scans were first performed on healthy volunteers. The local medical ethics committee approved all studies, and informed consents were obtained from all volunteers prior to the MR scan.

The four-channel phased array receive coil is interfaced to the scanner through a dedicated conversion box. During all scans, the commercial posterior array coil, which is built into the patient bed was electronically disconnected. In order to assess the performance of the receive coil array, scans on a cylindrical saline phantom (12 cm diameter) representing the neck were performed. The maximum acceleration factor that results in artifact-free images
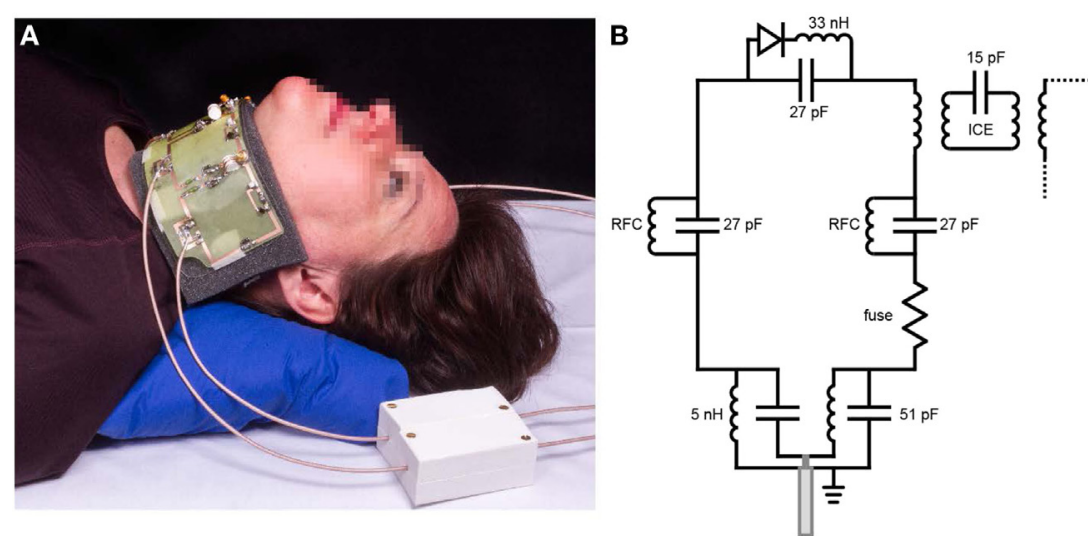

FIGURE 2 | (A) The dedicated larynx coil with the electronics visible (an encased version is used for in vivo scans). The coil is flexible and, therefore, adjustable to different neck sizes. The white boxes are floating cable traps, which prevent current from flowing on the outer shield of the connecting cables. Written informed consent was obtained from the subject for publication of this photo. (B) A schematic of the electrical circuit of each of the four loops constituting the coil. The individual loops are decoupled from the neighboring elements using an induced current elimination (ICE) circuit. Radiofrequency chokes (RFCs) are used to allow a DC current path to turn on the PIN diodes to isolate the receive coils from the transmit body coil. 
TABLE 1 | Scan parameters used for volunteer and patient studies.

\begin{tabular}{|c|c|c|c|c|c|}
\hline & T1-weighted TSE & $\begin{array}{l}\text { T1-weighted TSE (with } \\
\text { SPIR fat suppression) }\end{array}$ & T2-weighted Dixon TSE & T2-weighted Dixon TSE & DWI \\
\hline $\mathrm{TE} / \mathrm{TR}(\mathrm{ms})$ & $5.9 / 725$ & $5.9 / 649$ & $100 / 4680$ & $60 / 2410$ & $51 / 2038$ \\
\hline Scan plane & Transversal & Transversal & Transversal & Coronal & Transversal \\
\hline Voxel size $\left(\mathrm{mm}^{3}\right)$ & $1.0 \times 1.0 \times 1.0$ & $1.0 \times 1.0 \times 1.0$ & $1.0 \times 1.0 \times 1.0$ & $0.65 \times 0.81 \times 1.5$ & $3.0 \times 3.0 \times 3.0$ \\
\hline Field of view $\left(\mathrm{mm}^{3}\right)$ & $200 \times 200 \times 40$ & $200 \times 200 \times 40$ & $200 \times 180 \times 40$ & $200 \times 200 \times 60$ & $108 \times 280 \times 198$ \\
\hline Phase direction & Anterior-posterior & Anterior-posterior & Anterior-posterior & Right-left & Anterior-posterior \\
\hline Acceleration factor & 3.0 & 3.0 & $1.5^{\mathrm{b}}$ & 2.3 & 3.0 \\
\hline Averages & 1 & 1 & 2 & 1 & 1 \\
\hline Total duration ${ }^{a}$ (seconds) & 189 & 216 & 253 & 54 & 168 \\
\hline
\end{tabular}

${ }^{a}$ As all scans are respiratory triggered, scan time is dependent on the breathing pattern of the subject and the times shown are based on a breathing rate of once every $7 \mathrm{~s}$. TSE, turbo-spin echo; DWI, diffusion-weighted imaging; SPIR, spectral presaturation with inversion recovery.

${ }^{b}$ An acceleration factor of 1.5 was used during the scans of the two patient cases described in the results section of this paper. Since that time, the protocol has been changed such that all transversal T2-weigthed Dixon TSE scans are performed using acceleration factors of 3.0, reducing scan time to $126 \mathrm{~s}$.

was determined from these phantom scans. Acceleration factors of between 2 and 4 in either the anterior-posterior or right-left directions showed that fold-over artifacts at a factor of three are present but do not fold-in over the region of the larynx.

In order to address movement artifacts, respiratory-triggered scans were implemented, meaning that images are only obtained during a limited portion of the breathing cycle (in this case during exhaling), ensuring a stable position of the larynx during data acquisition. The respiratory trigger was applied using a belt on the abdomen, which triggered $500 \mathrm{~ms}$ after the initial stage of exhalation. Interestingly, since breathing and swallowing are physiologically linked (swallowing often occurs after exhaling), the use of respiratory-triggered scans also prevents swallowinginduced artifacts during image acquisition.

With respect to reducing image artifacts due to pulsatile blood flow in the arteries, we determined that implementing spatial saturation pulses caudal to the larynx did not result in a significant decrease in pulsation artifacts, and so were not used. Instead, to prevent pulsation artifacts falling within the larynx, the phase encoding direction was chosen to be in the anteriorposterior direction (23) for all transversal scans. For coronal scans, the anterior-posterior direction represents the slice-select direction for two-dimensional scans. The feet-head direction would be preferred for these scans, however, due to the geometry of the RF coil array acceleration cannot be obtained in this phase encoding direction. Right-left phase encoding is, therefore, the only possible phase encoding direction for coronal slices. This can sometimes result in pulsatile artifacts in the posterior part of the larynx.

\section{RESULTS}

\section{Healthy Volunteers}

A comparison between the setups shown in Figure 1A (two commercial loops) and Figure $\mathbf{2 A}$ (dedicated coil) is shown in Figure 3. The scan with the loops placed on a cooperative volunteer was acquired with an acceleration factor of two (the maximum achievable factor for a two-element coil), while the scan with the dedicated coil was performed with an acceleration factor of three. The image quality between the two setups is very similar (when the two commercial loops are perfectly positioned),

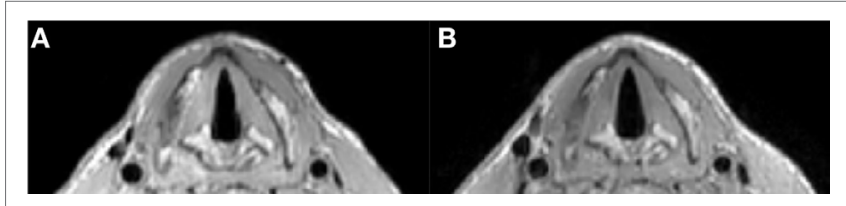

FIGURE 3 | Respiratory triggered T1 TSE sequence (1 mm isotropic voxel size) with parameters as in Table 1. (A) Shows an image at the level of the vocal cords made with the two loops setup (as in Figure 1A) and at the maximum acceleration factor of 2.0: total scan time $234 \mathrm{~s}$. (B) Shows an image at the same level made with the dedicated coil and an acceleration factor of 3.0: total scan time $189 \mathrm{~s}$.

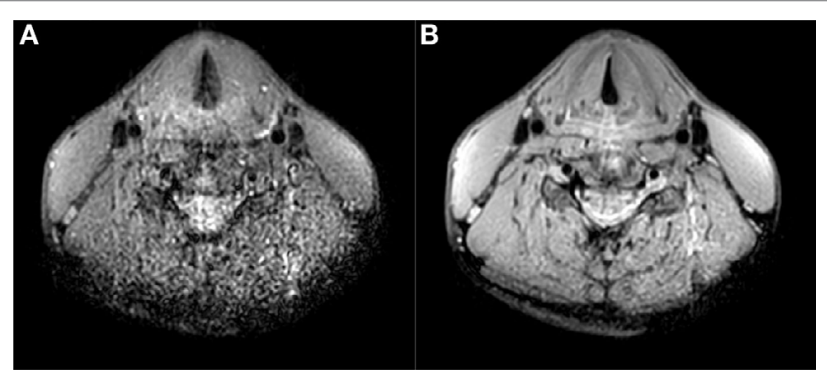

FIGURE 4 | T1 TSE multi-slice sequence (transversal) at $1.0 \mathrm{~mm}$ isotropic resolution with SPIR fat suppression (scan parameters in Table 1) (A) without respiratory trigger, duration $57 \mathrm{~s}$, (B) with respiratory trigger, duration $216 \mathrm{~s}$. Triggering mitigates breathing and swallowing motion artifacts and increases image quality.

but the scan with the dedicated coil could be performed with a $33 \%$ reduction in scan time. The images show that the dedicated coil outperforms the commercially available setup even under ideal scanning conditions.

Figure 4 shows a comparison of images acquired with and without respiratory triggering. Respiratory triggering obviously increases the total imaging time, but the acceleration factor of three using the dedicated coil array reduces the scan time to $3 \mathrm{~min}$ and results in images without breathing and swallowing motion artifacts while at the same time also improving general image quality. 


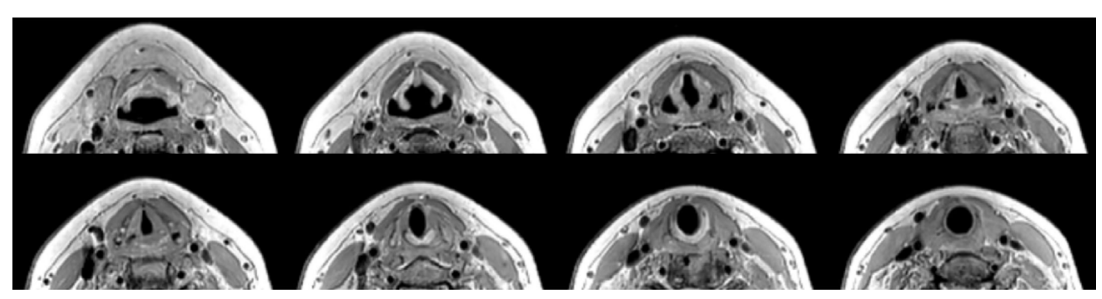

FIGURE 5 | T1 TSE $1.0 \mathrm{~mm}$ isotropic scan with parameters as in Table 1. A selection of eight out of 40 slices ranging from aryepiglottic fold to subglottis on a healthy volunteer showing depth of penetration far beyond the larynx.

With the dedicated larynx coil, the entire length of the larynx can be covered and also the penetration depth is more than sufficient for imaging the full larynx. In total, 11 volunteers have been scanned with this setup, showing similar image quality for all volunteers. An example of the field of view is shown is shown in Figure 5.

\section{Patient Scanning}

After scanning healthy volunteers for protocol optimization, scans were performed on two patients with suspected tumor recurrence after treatment. The full scan protocol for patients includes a transversal T1 TSE, transversal and coronal T2 TSE Dixon, transversal DWI sequence before gadolinium being administered and a set of transversal T1 TSE scans with SPIR (spectral presaturation with inversion recovery) fat suppression after gadolinium administration. This protocol takes less than $30 \mathrm{~min}$.

\section{Patient Case 1}

A 64-year-old male patient presented with a carcinoma of the left vocal cord with involvement of the anterior commissure and the anterior part of the contralateral vocal cord (T1b). Because of limited endoscopic exposure of the larynx, TLM could not be performed and the patient was treated with RT (25 fractions of $2.4 \mathrm{~Gy}$ each). Due to anxiety disorder, the patient refused flexible laryngoscopy during follow-up. For further surveillance, an MRI examination of the larynx was requested 8 months after irradiation. A subsection of the images acquired using the new coil and imaging protocols are shown in Figure 6. On T2-weighted images without and with fat suppression increased signal intensity was seen in both vocal cords and an area with intermediate signal on the surface of the left vocal cord (Figures 6A,B). On DWI, the T2 hyperintense areas showed no diffusion restriction, corresponding to inflammatory edema. DWI showed diffusion restriction in the superficial portion of the left vocal cord, compatible with submucosal recurrence (Figures 6C,D). In this patient, slowly increasing enhancement was seen and in tumor recurrence enhancement has been reported to be variable (8) (Figures 6E,F). Histopathological examination of biopsy specimens from the left vocal cord confirmed the presence of squamous cell carcinoma.

\section{Patient Case 2}

During follow-up of an 82-year-old male patient, who underwent RT for a T2 glottic carcinoma of the right vocal cord 1 year previously with sub- and supraglottic extension, an ulceration

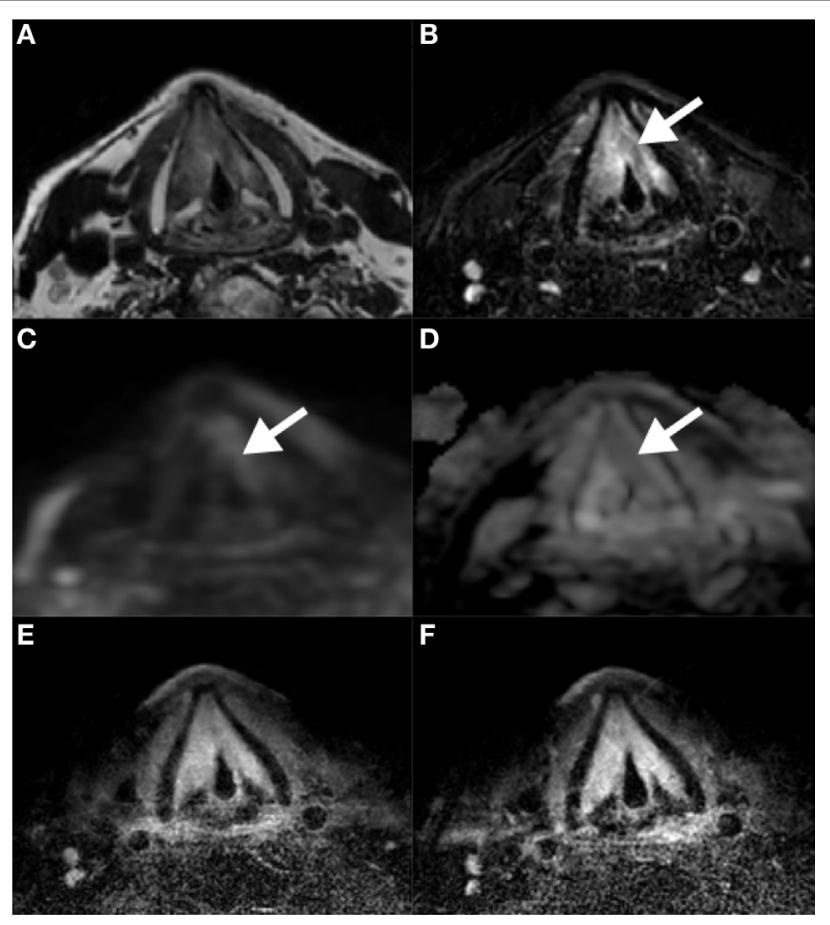

FIGURE 6 | Magnetic resonance imaging scans 8 months after radiation therapy for a T1b glottis carcinoma. Increased signal intensity on T2weighted scans without (A) and with (B) fat suppression in both vocal cords, together with the absence of diffusion restriction on DWI/ADC (C,D) is compatible with posttreatment edema. A biopsy in the superficial area corresponding to intermediate signal on T2 and diffusion restriction on the left vocal cord revealed tumor recurrence. Contrast-enhanced T1-weighted scans at two different time points show gradual enhancement (E,F).

suggestive of tumor recurrence was detected. The patient was referred for imaging to evaluate the depth of extension. Figure 7 shows four scans used for assessment using the new receive coil array and respiratory-triggered scans. Overall, the scans revealed a paramedian position of the right vocal cord and widening of the space between the thyroid cartilage and arytenoid. On the T2-weighted images, hyperintense signal is seen in the posterior portion of the vocal cord and in the posterior paraglottic space. This area showed enhancement, but no diffusion restriction. This MRI pattern is compatible with inflammatory changes or edema secondary to radiation therapy. Examination under anesthesia was performed and a biopsy of the superficial ulceration was taken. 


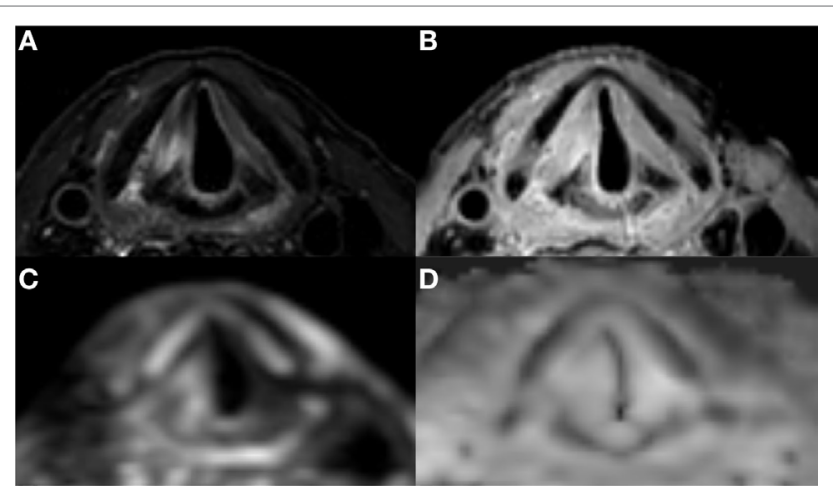

FIGURE 7 | Laryngeal magnetic resonance imaging of a patient with superficial recurrence after radiotherapy of an early glottis carcinoma: high signal intensity in the right vocal cord and posterior paraglottic space on T2 (A) combined with a high signal intensity on both DWI (C) and ADC (D) is compatible with post irradiation edema and rules out deep tumor extension. There is diffuse enhancement on contrast enhanced T1 with fat suppression (B).

The underlying thyroarytenoid muscle appeared free of tumor. Pathologic exam confirmed a superficial tumor recurrence.

\section{DISCUSSION}

Early and moderately advanced laryngeal carcinoma are well treatable diseases by the main treatment modalities of TLM, OPL, and RT, all of which have their relative benefits and limitations. In choosing and administering the most suitable treatment option for the individual patient, there are increasing demands for high quality imaging to: (1) assess image-based factors that determine the relative suitability for the different treatment modalities, (2) distinguish edema from tumor tissue, (3) predict the extent of the resection needed and the functional consequences, (4) identify early submucosal recurrence, and (5) improve tumor delineation in highly focused RT strategies. Key factors to obtain these high quality, artifact-free MR images of the glottis are to have a coil with high local sensitivity and to perform data acquisition with protocols, which reduce any motion-induced imaging artifacts. Reviewing previous literature, most groups use commercial general purpose surface coils. 3D gradient echo T1 sequences with fat suppression (for example, volumetric interpolated breath-hold examination) can be obtained with submillimeter voxels in cooperative patients $(8,18,20)$. For TSE sequences, slice thicknesses of $3-5 \mathrm{~mm}$ are chosen to reduce noise and acquisition time (11, 17, 18, 20-23).

Our main aim in this work is to improve on these previously reported scan protocols, particularly with respect to reduced slice thickness in TSE sequences. In order to produce artifact-free images, respiratory-triggered sequences were employed, which intrinsically increases the image acquisition time compared to non-triggered sequences. In order to at least partially compensate for this, an RF coil with high parallel imaging performance needed to be designed, which can be achieved by fabricating a coil with multiple individual elements. Many design criteria had to be taken into account and multiple prototypes have been built.
The coil had to be flexible and fit many different neck-sizes, while being robust to ensure that soldering of the electronic components was not compromised under flexation. With the final design consisting of four channels, it is able to scan fast with an acceleration factor of three and allows for imaging with adequate SNR using a slice thickness of only $1 \mathrm{~mm}$ and a voxel size of $1 \mathrm{~mm}^{3}$.

Having obtained considerable experience in scanning both healthy volunteers and patients using the dedicated larynx coil and respiratory-triggered acquisitions, we anticipate that many aspects of image acquisition are worth pursuing to improve the efficiency of data acquisition. For example, we have seen that some sequences do not fully use the available acquisition time during the exhalation. This unnecessarily lengthens these scans by requiring more breathing cycles for data acquisition. Other methods to obtain faster scans, other than parallel imaging, such as compressed sensing (27) have not yet been implemented and can result in substantial scan duration reduction.

In addition, it should be noted that the use of a respiratory trigger also creates extra constraints and challenges. For example, it requires the subject to have a regular breathing pattern that is not too slow, as a slow breathing pattern will slow down acquisition and lengthen the scans. Furthermore, when performing dynamic contrast enhanced scans, due to the lengthening of the scans with the respiratory trigger, early contrast enhancement may be missed. These scans should, therefore, not be performed using a trigger. A last point is the fact that we have initially chosen the $1.0 \mathrm{~mm}$ isotropic resolution for most scans in order to be able to easily reslice in any given direction. This reslicing has not always been successful, as very slight displacement between the slices is observed originating from some residual motion, resulting in staircasing of anatomical boundaries when performing multi-slice $2 \mathrm{D}$ acquisitions. For improving the performance of coronal scans, a coil with a higher spatial encoding power in the feet-head direction would be beneficial in order to obtain a better acceleration performance.

The dedicated coil has also been designed with integration with RT in mind. Patients receiving RT for laryngeal tumors are fixated in a customized individual mask. Fixation of the patient ensures treatment delivery reproducibility, as RT is delivered not in one but in many separate fractions. Because of the required reproducibility, the $\mathrm{CT}$ treatment planning is also done in a mask, as should be any diagnostic MR for RT purposes to allow for accurate image registration. In this way, the advantages of tumor delineation for RT based on a high-resolution MRI are fully utilized (28). Accurate tumor delineation is the first step of the RT process for a highly focused dose delivery, which is critical when aiming for voice preservation and less side effects such as dysphagia, xerostomia, or stroke related to the dose received by the carotid arteries. Our coil is the first dedicated laryngeal coil, to our knowledge, which has been designed to fit a RT mask (23).

\section{CONCLUSION}

The developed dedicated larynx coil and scan protocols allow for high resolution imaging ( $1.0 \mathrm{~mm}$ isotropic for TSE sequences) of the larynx, without being affected by breathing or swallowing 
artifacts. The coil allows efficient parallel imaging, which is used to speed up data acquisition. Furthermore, the coil is flexible and, therefore, easily accommodates different neck sizes. Additionally, the short total protocol time for patients (30 min maximum) and the fact that they can continue breathing and swallowing during the scans, reduces the burden of these scans for patients. We anticipate that this improved image quality will lead to better treatment planning and counseling in patients with laryngeal tumors.

\section{DATA AVAILABILITY STATEMENT}

Datasets are available on request: the raw (anonymized) data supporting the conclusions of this manuscript will be made available by the authors, without undue reservation, to any qualified researcher.

\section{ETHICS STATEMENT}

This study was carried out in accordance with the recommendations of the Medical Ethics Committee of the Leiden University Medical Center. The protocol was approved by the Medical

\section{REFERENCES}

1. Peretti G, Piazza C, Mora F, Garofolo S, Guastini L. Reasonable limits for transoral laser microsurgery in laryngeal cancer. Curr Opin Otolaryngol Head Neck Surg (2016) 24(2):135-9. doi:10.1097/MOO.0000000000000240

2. Peretti G, Piazza C, Del Bon F, Mora R, Grazioli P, Barbieri D, et al. Function preservation using transoral laser surgery for T2-T3 glottic cancer: oncologic, vocal, and swallowing outcomes. Eur Arch Otorhinolaryngol (2013) 270(8):2275-81. doi:10.1007/s00405-013-2461-9

3. Sjögren EV, van Rossum MA, Langeveld TP, Voerman MS, van de Kamp VA, Friebel MO, et al. Voice outcome in T1a midcord glottic carcinoma: laser surgery vs radiotherapy. Arch Otolaryngol Head Neck Surg (2008) 134(9):965-72. doi:10.1001/archotol.134.9.965

4. van Loon Y, Sjögren EV, Langeveld TP, de Jong B, Rob J, Schoones JW, et al. Functional outcomes after radiotherapy or laser surgery in early glottic carcinoma: a systematic review. Head Neck (2012) 34(8):1179-89. doi:10.1002/ hed. 21783

5. Hendriksma M, Heijnen BJ, Sjögren EV. Oncologic and functional outcomes of patients treated with transoral CO2 laser microsurgery or radiotherapy for T2 glottic carcinoma: a systematic review of the literature. Curr Opin Otolaryngol Head Neck Surg (2018) 26(2):84-93. doi:10.1097/ MOO.0000000000000438

6. Schrijvers ML, van Riel EL, Langendijk JA, Dikkers FG, Schuuring E, van der Wal JE, et al. Higher laryngeal preservation rate after CO2 laser surgery compared with radiotherapy in T1a glottic laryngeal carcinoma. Head Neck (2009) 31(6):759-64. doi:10.1002/hed.21027

7. Mahler V, Boysen M, Brøndbo K. Radiotherapy or CO2 laser surgery as treatment of Tla glottic carcinoma? Eur Arch Otorhinolaryngol (2010) 267(5):743-50. doi:10.1007/s00405-009-1135-0

8. Ravanelli M, Farina D, Rizzardi P, Botturi E, Prandolini P, Mangili S, et al. MR with surface coils in the follow-up after endoscopic laser resection for glottic squamous cell carcinoma. Neuroradiology (2013) 55(2):225-32. doi:10.1007/s00234-012-1128-3

9. Osman SO, Astreinidou E, de Boer HC, Keskin-Cambay F, Breedveld S, Voet P, et al. IMRT for image-guided single vocal cord irradiation. Int J Radiat Oncol Biol Phys (2012) 82(2):989-97. doi:10.1016/j.ijrobp.2010.12.022

10. Grégoire V, Evans M, Le QT, Bourhis J, Budach V, Chen A, et al. Delineation of the primary tumour Clinical Target Volumes (CTV-P) in laryngeal,
Ethics Committee of the Leiden University Medical Center. All subjects gave written informed consent in accordance with the Declaration of Helsinki.

\section{AUTHOR CONTRIBUTIONS}

TR built the MR coil. TR, BV, and JO developed the MR scan protocols and performed the scans. TR, BV, ES, EA, and AW have written the manuscript. All authors contributed to the scientific debate.

\section{ACKNOWLEDGMENTS}

The authors would like to thank Paul W. de Bruin for setting up the initial scan protocols and for his survey on different hardware setups for imaging the larynx.

\section{FUNDING}

This work was supported by The Netherlands Organization for Scientific Research, domain Applied and Engineering Sciences under grant number 13783.

hypopharyngeal, oropharyngeal and oral cavity squamous cell carcinoma: AIRO, CACA, DAHANCA, EORTC, GEORCC, GORTEC, HKNPCSG, HNCIG, IAG-KHT, LPRHHT, NCIC CTG, NCRI, NRG Oncology, PHNS, SBRT, SOMERA, SRO, SSHNO, TROG consensus guidelines. Radiother Oncol (2018) 126(1):3-24. doi:10.1016/j.radonc.2017.10.016

11. Ravanelli M, Agazzi GM, Farina D, Maroldi R. New developments in imaging of laryngeal cancer. Curr Otorhinolaryngol Rep (2017) 5(1):49-55. doi:10.1007/ s40136-017-0145-5

12. Castelijns JA, Doornbos J, Verbeeten JB, Vielvoye GJ, Bloem JL. MR imaging of the normal larynx. J Comput Assist Tomogr (1985) 9(5):919-25. doi:10.1097/00004728-198509000-00015

13. Curtin HD. Imaging of the larynx: current concepts. Radiology (1989) 173(1):1-11. doi:10.1148/radiology.173.1.2675175

14. Lufkin RB, Hanafee WN. Application of surface coils to MR anatomy of the larynx. AJR Am J Roentgenol (1985) 145(3):483-9.

15. McArdle CB, Bailey BJ, Amparo EG. Surface coil magnetic resonance imaging of the normal larynx. Arch Otolaryngol Head Neck Surg (1986) 112(6):616-22. doi:10.1001/archotol.1986.03780060028003

16. Pruessmann KP, Weiger M, Scheidegger MB, Boesiger P. SENSE: sensitivity encoding for fast MRI. Magn Reson Med (1999) 42(5):952-62. doi:10.1002/ (SICI) 1522-2594(199911)42:5<952::AID-MRM16>3.0.CO;2-S

17. Casselman JW. High resolution imaging of the skull base an larynx. In: Schoenberg SO, Dietrich O, Reiser MF, editors. Parallel Imaging in Clinical MR Applications. Berlin, Heidelberg, New York: Springer Science \& Business Media (2007). p. 199-208.

18. Maroldi R, Ravanelli M, Farina D. Magnetic resonance for laryngeal cancer. Curr Opin Otolaryngol Head Neck Surg (2014) 22(2):131-9. doi:10.1097/ MOO.0000000000000036

19. Becker M, Burkhardt K, Dulguerov P, Allal A. Imaging of the larynx and hypopharynx. Eur J Radiol (2008) 66(3):460-79. doi:10.1016/j.ejrad.2008. 03.027

20. Preda L, Conte G, Bonello L, Giannitto C, Tagliabue E, Raimondi S, et al. Diagnostic accuracy of surface coil MRI in assessing cartilaginous invasion in laryngeal tumours: do we need contrast-agent administration? Eur Radiol (2017) 27(11):4690-8. doi:10.1007/s00330-017-4840-x

21. Allegra E, Ferrise P, Trapasso S, Trapuzzano O, Barca A, Tamburrini S, et al. Early glottic cancer: role of MRI in the preoperative staging. Biomed Res Int (2014) 2014:890385. doi:10.1155/2014/890385 
22. Jager EA, Kasperts N, Caldas-Magalhaes J, Philippens ME, Pameijer FA, Terhaard $\mathrm{CH}$, et al. GTV delineation in supraglottic laryngeal carcinoma: interobserver agreement of CT versus CT-MR delineation. Radiat Oncol (2015) 10(1):26. doi:10.1186/s13014-014-0321-4

23. Verduijn GM, Bartels LW, Raaijmakers CP, Terhaard CH, Pameijer FA, van den Berg CA. Magnetic resonance imaging protocol optimization for delineation of gross tumor volume in hypopharyngeal and laryngeal tumors. Int J Radiat Oncol Biol Phys (2009) 74(2):630-6. doi:10.1016/j.ijrobp.2009.01.014

24. Roemer PB, Edelstein WA, Hayes CE, Souza SP, Mueller OM. The NMR phased array. Magn Reson Med (1990) 16(2):192-225. doi:10.1002/mrm.1910160203

25. Yan X, Gore JC, Grissom WA. New resonator geometries for ICE decoupling of loop arrays. J Magn Reson (2017) 277:59-67. doi:10.1016/j.jmr.2017.02.011

26. Seeber DA, Jevtic J, Menon A. Floating shield current suppression trap. Concepts Magn Reson B (2004) 21(1):26-31. doi:10.1002/cmr.b.20008

27. Lustig M, Donoho D, Pauly JM. Sparse MRI: the application of compressed sensing for rapid MR imaging. Magn Reson Med (2007) 58(6):1182-95. doi:10.1002/mrm.21391
28. Hanvey S, McJury M, Tho LM, Glegg M, Thomson M, Grose D, et al. The influence of MRI scan position on patients with oropharyngeal cancer undergoing radical radiotherapy. Radiat Oncol (2013) 8(1):129. doi:10.1186/1748717X-8-129

Conflict of Interest Statement: All authors declare that the research was conducted in the absence of any commercial or financial relationships that could be construed as a potential conflict of interest.

Copyright (c) 2018 Ruytenberg, Verbist, Vonk-Van Oosten, Astreinidou, Sjögren and Webb. This is an open-access article distributed under the terms of the Creative Commons Attribution License (CC BY). The use, distribution or reproduction in other forums is permitted, provided the original author(s) and the copyright owner are credited and that the original publication in this journal is cited, in accordance with accepted academic practice. No use, distribution or reproduction is permitted which does not comply with these terms. 


\section{OPEN ACCESS}

Edited by:

Vincent Vander Poorten,

KU Leuven, Belgium

Reviewed by:

Giuseppe Mercante,

National Cancer Institute Regina

Elena, Italy

Georgios Psychogios,

Hospital Augsburg, Germany

*Correspondence:

Yaniv Hamzany

yaniv@hamzany.com

Specialty section:

This article was submitted to

Head and Neck Cancer

a section of the journal

Frontiers in Oncology

Received: 15 April 2018

Accepted: 17 July 2018

Published: 14 August 2018

Citation:

Hamzany Y, Shoffel-Havakuk H,

Devons-Sberro S, Shteinberg S, Yaniv D and Mizrachi A (2018) Single

Stage Transoral Laser Microsurgery for Early Glottic Cancer.

Front. Oncol. 8:298.

doi: 10.3389/fonc.2018.00298

\section{Single Stage Transoral Laser Microsurgery for Early Glottic Cancer}

\author{
Yaniv Hamzany ${ }^{1,2 *}$, Hagit Shoffel-Havakuk ${ }^{1,2}$, Stav Devons-Sberro ${ }^{3}$, Shani Shteinberg ${ }^{2,4}$, \\ Dan Yaniv ${ }^{1,2}$ and Aviram Mizrachi ${ }^{1,2}$ \\ ${ }^{1}$ Department of Otolaryngology Head and Neck Surgery, Rabin Medical Center, Petach Tikva, Israel, ${ }^{2}$ Sackler Faculty of \\ Medicine, Tel Aviv University, Tel Aviv, Israel, ${ }^{3}$ Hadassah-Hebrew University School of Medicine, Jerusalem, Israel, ${ }^{4}$ Institute \\ for Speech, Swallowing and Voice Rehabilitation, Rabin Medical Center, Petach Tikva, Israel
}

Objectives: The purpose of the study was to present the outcome of our management protocol of a single stage transoral laser microsurgery (SSTLM), with the intention of complete removal of a lesion, considered to be an early glottic cancer.

Methods: Between January 2015 to February 2017 patients with the clinical appearance of an early glottic cancer, who were candidates for (SSTLM) management protocol, were included in this study. Type of cordectomy was determined by pre- and intra-operative evaluation of the extent of lesion in cord layers.

Results: Thirty patients (6 females, 24 males; mean age 65 years) underwent SSTLM. Twenty-two patients had malignant histopathological diagnosis of severe dysplasia or Cis in 4 patients, microinvasice carcinoma in 3 patients and invasive carcinoma in 15 patients (T1a tumor in 14 and T1b tumor in 1). Eight patients had a nonmalignant histological diagnosis of keratosis without atypia in 2 patients, mild dysplasia in 2 patients and moderate dysplasia in 3 patients. Based on pre- and intra-operative evaluation, 14 subepithelial (type I), 10 subligamental (type II), and 6 transmuscular (type III) cordectomies were performed. Comparison of cordectomies types with postoperative histopathologic diagnosis showed an adequate extent of resection in 26 out of 30 patients (87\%). Considering only patients without recent background of direct laryngoscopy and biopsy, an adequate resection was performed in $90 \%$ of patients. None of the patients was further treated by external beam radiation. At average follow-up of 21 months, none of the patients developed local recurrence.

Conclusion: In selected cases, a SSTLM for clinical appearance of an early glottic cancer, allows a reliable histopathologic diagnosis and a high local control rate with favorable cost effectiveness. A careful pre- and intraoperative evaluation for selecting the appropriate cases for this management is required in order to avoid under- or over-treatment.

Keywords: glottic cancer, $\mathrm{CO}_{2}$ laser, excisional biopsy, single stage, transoral laser microsurgery, complete removal

\section{INTRODUCTION}

The histological diagnosis of laryngeal epithelial lesions may range from benign (including hyperplasia, keratosis) to premalignant lesions [including dysplasia and carcinoma in situ (Cis)] to invasive squamous cell carcinoma (SCC) (1). Precise evaluation of suspected glottic lesion requires careful endoscopic visualization and tissue sampling. 
However, performing incisional biopsy, which is not representative of the entire lesion, for diagnostic purposes, has several disadvantages. While superficial biopsies, might result in inadequate sampling, or could miss the diagnosis of invasion, deep biopsies may result in inflammation and fibrotic changes at the epithelium-vocal ligament interface, these can cause an unclear borders appearance, or limit a proper evaluation of the deep extent of the residual tumor. A larger unnecessary resection might be performed in a subsequently curative procedure, potentially compromising voice outcome (2). Furthermore, in up to $33 \%$ of patients with early glottic cancer, complete tumor removal is achieved with initial biopsy, leading to unnecessary additional treatment $(3,4)$. Therefore, in selected cases of early glottis cancer, a careful excisional biopsy with complete removal of the entire lesion with a limited rim of healthy tissue, at the first direct laryngoscopy (DL), may provide an unequivocal diagnosis along with curative treatment and improved voice outcome.

Blakeslee et al. comprehensive report from 1984 presented the concept of $\mathrm{CO}_{2}$ laser single stage excisional biopsy in the selective management of T1 glottic cancer (5). Later publication by Ossoff et al., Peretti et al., Sagiston et al. and Manola et al. (2, 6-9) supported this management for Tis and T1, mostly in cases of superficial or limited lesions. Nowadays, endoscopic resection of T1 glottic carcinoma is a valid standard of care however, a review of the literature reveals a lack of recent publications discussing indications, principles and results of a single stage DL with the intention of complete removal of a lesion considered to be an early glottic cancer. This study aims to present the outcome of our management protocol of a single stage transoral laser microsurgery (SSTLM), with curative intent, for early glottic cancer.

\section{MATERIALS AND METHODS}

Patients with glottic lesions, candidates for SSTLM management protocol at the department of Otolaryngology Head and Neck Surgery of Rabin Medical Center (as will be further described in detail), between January 2015 to February 2017, were included in the study. Following an approval by the institutional ethics committee the medical records of included patients were reviewed and data regarding demographics, diagnosis, pathology, outcome, voice quality, and follow-up was collected. Outcome measures included: staging at diagnosis, type of cordectomy, histopathological diagnosis, voice evaluation, further treatment and recurrence rate.

\section{Management Protocol for SSTLM}

Before being selected for this approach, all patients had to meet the following criteria.

The lesion had to have the common characteristic appearance raising a high suspicion for early glottic cancer (e.g., keratosis, irregularity, infiltration, vascularity) under careful endoscopic evaluation at the clinic by 70 -degree rigid endoscope with or without videostroboscopy. The lesion had to be confined to the true vocal cords with preserved vocal cords motion. In patients with suspected deep infiltration of the vocal cord, involvement of anterior commissure, body of arytenoid, lesion extending to the superior arcuate line into the ventricle, or subglottic or supraglottic expansion, a CT scan with intravenous contrast agent was obtained. Evidence for involvement of the paraglottic fat, thyroid cartilage, body of the arytenoid, supraglottic or subglottic regions, was contraindication to SSTLM. In such cases patient underwent incisional biopsy, either by DL under general anesthesia, or by using a distal chip camera video nasolaryngoscope with a working channel under local anesthesia as an office procedure.

Patients had to be medically approved for endoscopic surgery under general anesthesia. Patients treated with antiplatelet or anticoagulation agents, were asked to discontinue the medications prior to surgery. Before signing an informed consent form, the patients received a detailed explanation about treatment options-a DL with biopsies for diagnostic purpose, being followed by a TLM or radiation therapy in case of a malignant lesion, or a SSTLM emphasizing the possibility that it might encompass an over resection influencing their voice outcome.

\section{Surgical Technique}

Patients were intubated using a small bore, laser safe with a double cuff, endotracheal tube (usually 5.0-6.0 mm ID). The larynx was exposed as widely as possible with suspension laryngoscopy using different shapes and sizes of laryngoscopes as needed. Counterpressure on the cricoid cartilage was performed as required for optimal visualization of the anterior commissure. Meticulous evaluation of the larynx was performed using a rigid 0 and 70-degree endoscopes (Figure 1). After achieving an adequate exposure of the glottis, microscopic examination with microinstrumantal palpation was undertaken. Surgery was performed under operating microscope, using both microsurgical instruments and $\mathrm{CO}_{2}$ Laser.

When needed vestibulectomy (i.e., resection of a false vocal cord or petiole of epiglottis) using $\mathrm{CO}_{2}$ laser was employed, in order to improve lesion exposure. After obtaining complete exposure of the lesion, including margins of healthy appearing mucosa, an adrenaline solution diluted by saline, at a concentration of 1: 100,000, was injected into Reinke's space for hydrodissection. A complete mucoligamentous hydrodissection indicated a lesion limited to the mucosa, that may be eligible for subepithelial (type I) cordectomy. If adherences were observed, indicating a lesion suspicious of involvement of the deeper layers of the vocal cord, subligamental (type II) or transmuscular (type III) cordectomy was performed corresponding with the depth of invasion as appearing under the operating microscope. $\mathrm{CO}_{2}$ laser resection was commenced by outlining margins of resection including a healthy mucosa of about $2 \mathrm{~mm}$ surrounding the lesion. Subsequently, an en-block resection was carried out in all cases. A careful clinical assessment of the margins of resection with the operating microscope and 0 and 70-degree endoscopes both during the resection and immediately on completion was done. 

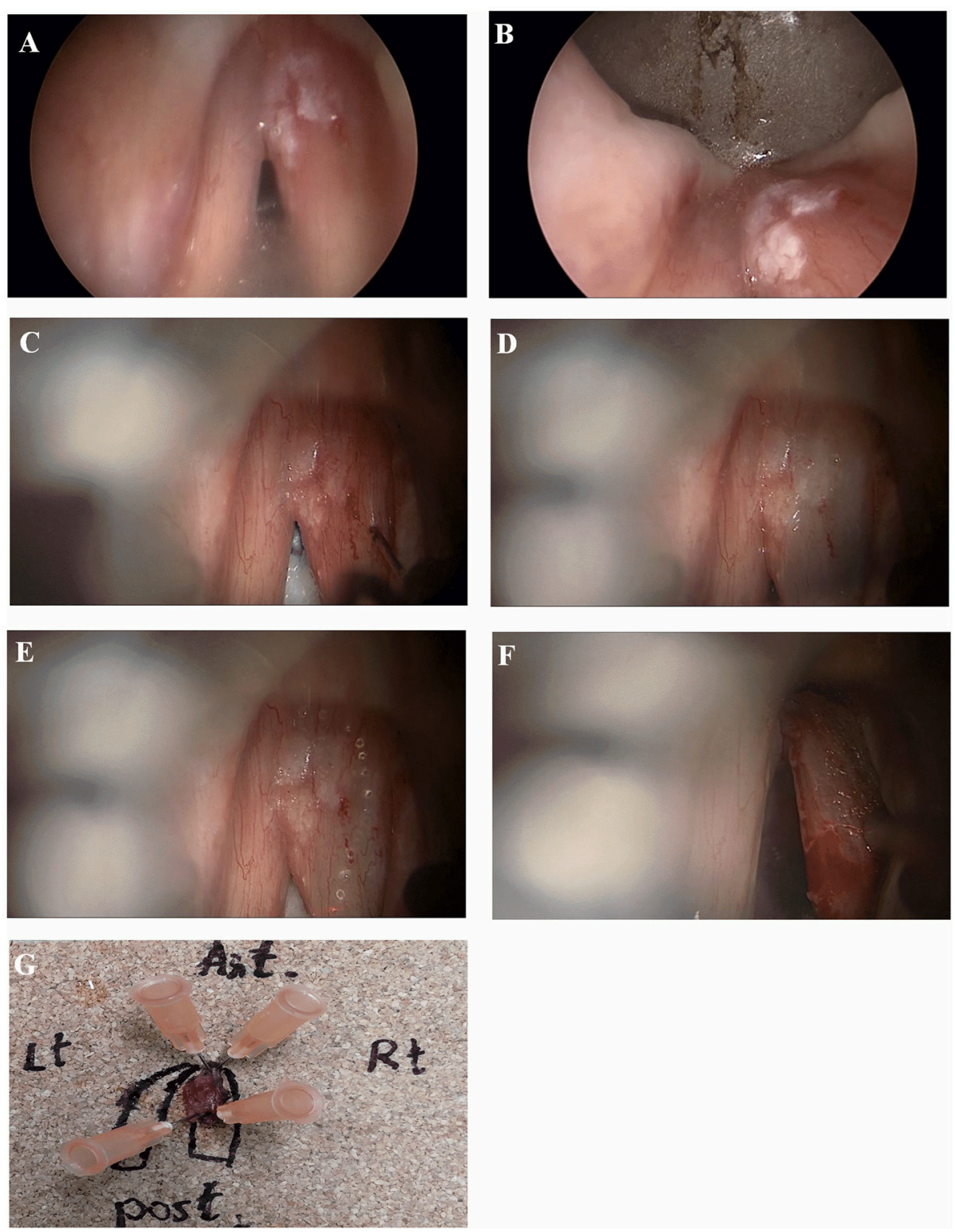

FIGURE 1 | Microinvasive SCC of the right vocal cord. (A, B) Endoscopic view with 0 and 70-degree endoscopes. Note limited involvement of anterior commissure, no extension into ventricle or subglottic area. (C, D) Microscopic view before and after mucoligamentous hydrodissection by injection of saline solution into Reinke's space. Note no adherence of the mucosa to the ligament, suggesting lesion limited to the mucosa. (E) A narrow mucosal margin has been made with the laser. (F) After type I cordectomy, the deep plane of excision is the vocal ligament, which is clearly exposed. (G) Pathological specimen pinned on a carrier substance, oriented, and mapped by marking directions and surrounding glottic structures.

In case of a lesion involving both vocal cords crossing through the anterior commissure, in order to avoid postoperative anterior synechiae, a staged bilateral cordectomy was performed; the second procedure was performed after 6-8 weeks.

Cordectomy was performed using $\mathrm{CO}_{2}$ laser (Lumenis Laser, Yokneam, Israel) with superpulse delivery in a continuous mode (1-3 W, 270-micron spot size), coupled to an operating microscope. Transoral laser cordectomy was classified according to the European Laryngological Society (10).

\section{Resection Margins Evaluation}

For histological evaluation, the surgical specimen was pinned on a carrier substance (cork board) and oriented and mapped by marking directions and surrounding glottic structures. After completion resection of the lesion, superficial and deep biopsies (sized 1-2 mm each) were obtained from the surgical bed of resection within the vocal cord (patient margins). Resection margins evaluation was based on margins at the pathological specimens and on patient margins. In case of positive margin at the specimens, but negative patient margins, patient was 
scheduled for close follow-up at the clinic every 1-2 months. In case of positive patient margin, additional TLM was performed with re-resection of involved margin.

\section{Post-operative Management and Follow-Up}

Soft oral intake was started on the operative day, and patients were discharged on the first or second postoperative day.

All patients were postoperatively examined every 2 months by a 70-degree rigid endoscope or a flexible fiberoptic endoscope. In the case of clinical suspicion of persistent or recurrent disease, a $\mathrm{CO}_{2}$ laser re-excision was performed.

\section{Voice Evaluation}

Patients participating in the study underwent a voice evaluation which took place at least 6 months after their last cordectomy. During voice evaluation, patients were administered with an established Hebrew version of the Voice-Related Quality of Life (V-RQOL) questionnaire (11). For perceptual voice evaluation, a standardized voice recording was performed during that visit using a head-mounted microphone placed $4 \mathrm{~cm}$ from the mouth. The voice recording protocol included reading of a standardized passage, sustained phonation of the vowels /a/ and /i/, load phonation and a glissando from low to high note. These voice recordings were used to determine the clinicians' perceptual voice evaluation using the GRBAS (grade, roughness, breathiness, asthenia, and strain) scale (12). A team of two clinicians experienced in voice disorders, blinded to the patient's identity, characteristics, and type of cordectomy, rated each voice sample according to the GRBAS scale. Acoustic analysis included fundamental frequency (F0), jitter, shimmer, and noiseto-harmonic ratio (NHR) was conducted with the Praat Voice Program (Paul Boersma and David Weenink, Phonetic Sciences, University of Amsterdam, Amsterdam, The Netherlands, version 6.0.36) using a sustained /a/ with a sample frequency of $44,000 \mathrm{~Hz}$. Maximum phonation time (MPT) was recorded and timed during sustained /a/ phonation at a comfortable intensity and pitch level on a single breath. Patients were instructed to take a deep breath before phonating and to sustain their phonation as long as possible. The best score out of 3 trials was used for analysis.

\section{Data Collection and Analysis}

Categorical variables are described by frequency and percentage; continuous variables are described by mean and standard deviation.

\section{RESULTS}

Between January 2015 to February 2017, 33 patients presented with clinical appearance of an early glottic cancer and were candidates for SSTLM at the Department of Otolaryngology Head and Neck Surgery of Rabin Medical Center. No initial biopsy from vocal cord was performed. Three out of 33 patients underwent DL with incisional biopsy due to inadequate exposure. Their final histopathological diagnosis was SCC and subsequently they were treated with external beam radiation therapy. Of the 30 patients who underwent SSTLM (6 females, 24 males; mean age 65; range 44-86 years), twenty-two had malignant histopathological diagnosis of severe dysplasia or Cis in 4 patients, microinvasice carcinoma in 3 patients and invasive carcinoma in 15 patients (T1a tumor in 14 and T1b tumor in 1; Table 1) (13). Five patients had anterior commissure involvement: 1, Cis; 3, T1a tumors; and 1, T1b tumor. Eight patients had a nonmalignant histological diagnosis of keratosis without atypia in 2 patients, mild dysplasia in 2 patients and moderate dysplasia in 3 patients. Based on the aforementioned criteria, 14 subepithelial (type I), 10 subligamental (type II), and 6 transmuscular (type III) cordectomies were performed. The type of cordectomy with regard to postoperative histopathological diagnosis is reported in Table 2.

Staged bilateral cordectomy was performed in one patient with T1b carcinoma. One patient underwent $\mathrm{CO}_{2}$ laser reexcision due to superficial patient margin positive for Cis. Histopathology of re-excision showed severe dysplasia. None of the patients was further treated by external beam radiation.

The average follow-up was at 21 months, ranging from 12 to 36 months. None of the patients developed local recurrence within the follow-up period. There has been one death of

TABLE 1 | Clinical demographic and histological findings.

Malignant $(n=22)$

Nonmalignant $(n=8)$

Gender

Male

Female

Age

Mean

Range

Smoking

Histological result
Severe dysplasia or Cis

microinvasive

Invasive carcinoma
Keratosis without atypia

Mild dysplasia

Moderate dysplasia
3

15

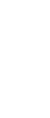

0 
TABLE 2 | Type of cordectomy according to histopathologic diagnosis.

\begin{tabular}{|c|c|c|c|c|c|}
\hline Histopathology & Patient no. & Type I & Type II & Type III & Over- treatment \\
\hline Keratosis, mild or moderate dysplasia & 8 & 5 & 3 & - & 3 \\
\hline Severe dysplasia or carcinoma in situ & 4 & 3 & 1 & - & 1 \\
\hline Microinvasive carcinoma & 3 & 3 & - & - & - \\
\hline Invasive Carcinoma & 15 & 3 & 6 & 6 & - \\
\hline Total & 30 & 14 & 10 & 6 & 4 \\
\hline
\end{tabular}

unrelated cause 22 months after TLM secondary to breast cancer. There have been no intraoperative or postoperative complications in any of the patients.

Of the 22 patients with a histological diagnosis of malignancy, except one patient who died of a cause unrelated to her laryngeal disease, all completed the post-operative voice evaluation. The timing of the post-operative voice evaluation ranged from 6 to 36 months after surgery, with a median of 12 months (mean $\pm \mathrm{SD} ; 16.0 \pm 10.2$ ). Detailed presentation of the post-operative voice evaluation can be found on (Table 3). In general, all patients demonstrated good voice outcome results. Patients who underwent type I cordectomy had better voice outcomes than patients who underwent type II or III cordectomies, for all measures examined in our study. None of the patients had any complaints of dysphagia or sustained aspiration.

\section{DISCUSSION}

Incisional random biopsies, solely for diagnosis of glottic lesions, have several disadvantages. Small or superficial random biopsies can be insufficient for diagnosis or might not be taken from the malignant area of the lesion, hence leading to under diagnosis. Conversely, a more extensive biopsy, may result in complete removal of the tumor. Nassif et al. and Zapater et al. published their results on negative pathology following endoscopic resection of $\mathrm{T} 1$ squamous carcinoma of the glottis $(3,4)$. They found that following initial biopsy of an invasive SCC of the glottis, there was negative pathology in up to $33 \%$ of patients who underwent a second surgery for complete tumor resection. Thus, in a considerable number of patients the tumor is completely removed unintentionally with the initial biopsy taken, and further surgery or external beam radiation treatment is unnecessary.

SSTLM with complete removal of the entire lesion with a limited rim of healthy tissue can provide an unequivocal diagnosis along with curative treatment. Moreover, in doing so, the best compromise between oncological aspect, and voice outcome can be achieved. Performing a narrow-margin cordectomy for T1 glottic carcinoma has already been proved to be oncologically safe, provided close follow-up is undertaken, with local control rate of $93-100 \%$ at 3 years $(2,9,14-18)$.

Evaluation of a glottic lesion and its borders done by DL under general anesthesia is more accurate before any biopsy is taken. Following vocal cord biopsy, the healing process causes inflammation and fibrosis which can blur the true borders between the lesion to the healthy mucosa, leading to a more extended resection during second procedure. Fibrosis and scarring of the superficial lamina propria (SLP) impairs the ability of the surgeon to assess deep involvement of the lesion beyond the mucosa. Failure to achieve a mucoligamentous separation when using the hydrodissection technique, or simply adherence of the ill mucosa to the ligament during the cordectomy, might result in resection of uninvolved deeper layers of the vocal cord.

Decision taking regarding the definitive treatment of suspected glottic lesions which solely relies on its endoscopic appearance is insufficient. Therefore, the management protocol of SSTLM requires a careful pre- and intraoperative evaluation, as described, in order to minimize the rate of under- and overtreatment cases. Peretti et al. described their experience with a pre- and intraoperative evaluation by video-laryngostroboscopy and saline infusion into Reinke's space in 52 patients with vocal cord erythroleukoplakias to predict the invasion of the layered structure of the lamina propria and consequently determine the depth of the excisional biopsy that should be carried out (19). They found that 38 patients underwent the appropriate type of resection, while 1 patient an undertreatment resection and 13 an overtreatment resection (10 of them were patients who had previous random biopsies). The authors of this publication stressed that in patients who had previous random biopsies done, the possible presence of scar tissue and fibrotic changes at the epithelium-vocal ligament interface limit the reliability of video-laryngostroboscopy and saline infusion information regarding the depth of lesion invasion. Other authors also reported that findings during videolaryngostroboscopy such as, mucosal wave absence or impaired progression correlated well with an incomplete mucoligamentous hydrodissection, thereby suggesting infiltration of the lamina propria $(20,21)$.

In our study, cordectomy type was mainly determined by intraoperative evaluation including hydrodissection technique and extent of tumor involvement of cord layers as observed during the resection. Comparison of cordectomies types with postoperative histopathologic diagnosis, showed a selection of the adequate treatment extent in 26 out of 30 patients (87\%). None of the patients received an undertreatment. Four patients were overtreated by subligamental cordectomy (type II) while in fact could have been safely treated by subepithelial cordectomy (type I). One of these patients underwent direct laryngoscopy with vocal cord biopsies, 7 months previously, with benign histological results, possibly impairing the evaluation of the deep extent of the lesion in cord layers. Excluding this patient, an overtreatment was performed in 3 of 29 patients (10\%) who underwent SSTLM, while an adequate resection was performed in $90 \%$. 
TABLE 3 | Post-operative voice evaluation and acoustic analysis results.

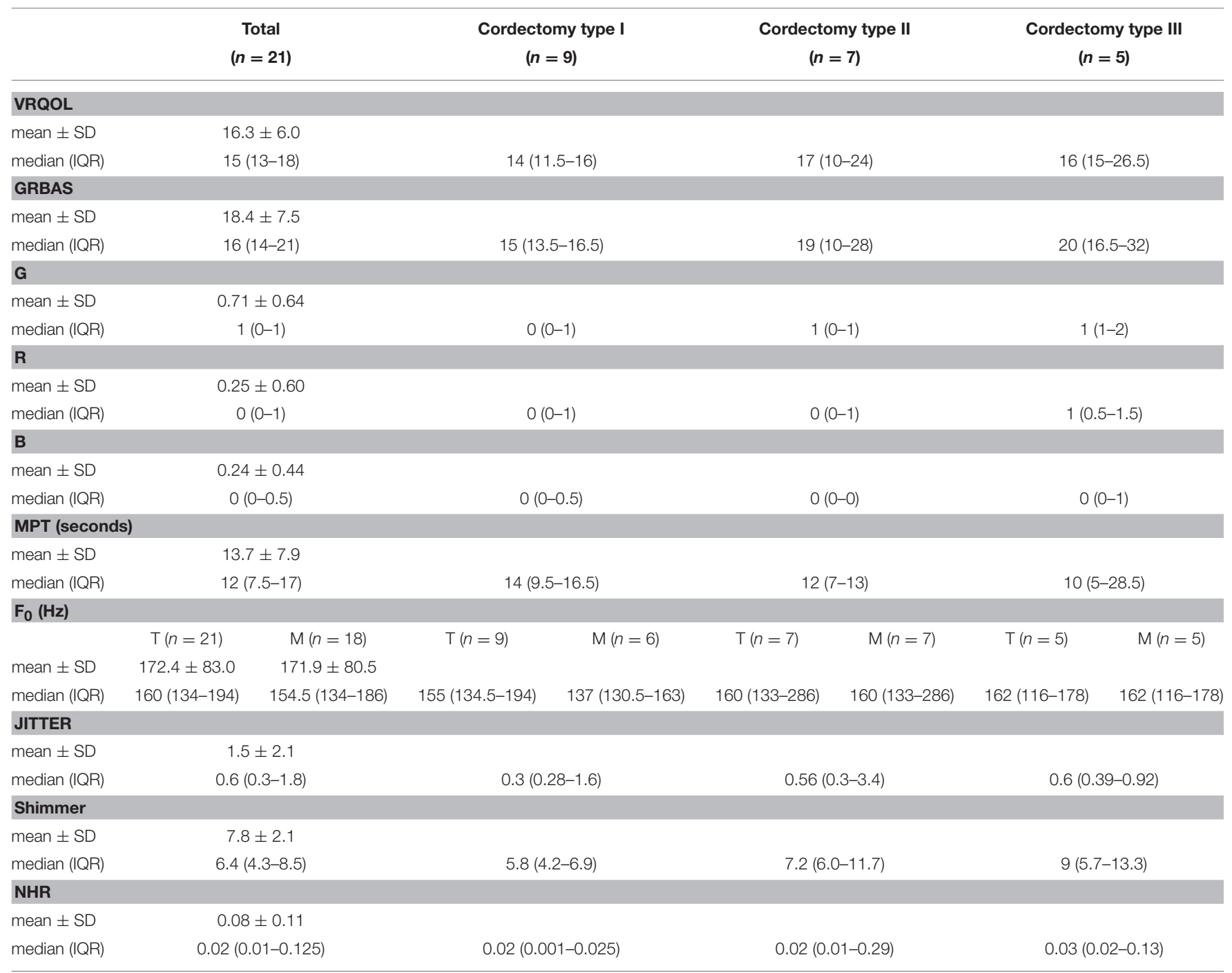

VRQOL, Voice Related Quality of Life raw (non-converted) values; SD, Standard Deviation; IQR, InterQuartile Range; G, Grade (GRBAS scale); R, roughness (GRBAS scale); B, breathiness (GRBAS scale); MPT, Maximum Phonation Time; Fo, Fundamental frequency; NHR, Noise to Harmonic Ration; T, Total; M, Male.

The concept of SSTLM for early glottic cancer was introduced by Blakeslee et al. as early as in the 1980s (5). They reported on 50 patients with T1 glottic cancer, of whom $46(92 \%)$ patients were treated successfully by transoral excisional biopsy alone, thus concluding that this management can unequivocally establish the diagnosis and stage of the disease and is adequate treatment for small glottic SCC.

Peretti et al. first reported on their experience with endoscopic laser excisional biopsy for selected glottic carcinoma in 1994, limiting this treatment concept to patients without anterior commissure involvement (7). Later publication, from 2001, described their management of SSTLM in 88 patients (13 Tis, $75 \mathrm{~T} 1)$ treated by cordectomy types $\mathrm{I}-\mathrm{V}$ (2). Patients with superficial involvement of anterior commissure without cartilage invasion by $\mathrm{CT}$ scan were included. Five-year local control rate was $91 \%$. Their results showed that SSTLM is an acceptable management also for infiltrative glottic cancer or cases involving the anterior commissure.

Sagiston et al. published their oncological results of laser cordectomy for early glottic cancer (8). Although detailed intraoperative evaluation and surgical technique consisted with those previously described, single stage excisional biopsies were performed only for cases of superficial lesions, by cordectomy types I-II. Their results suggest that subligamental (type II) cordectomy may be an adequate treatment for Tis or very small T1a tumors.

Manola et al. performed 29 partial cordectomies and two complete cordectomies at first operation, in 31 patients with $\mathrm{T} 1$ glottic carcinoma (9). Local control rate was $95 \%$. They comment that when preoperative and intraoperative work-up is respected, the risk of under- and over-treatment is very low.

In our study, only one patient with superficial patient margin positive for Cis, underwent $\mathrm{CO} 2$ laser re-excision, of 
which histopathology showed severe dysplasia. None of the patient developed local recurrence during the follow-up period. Although our management did not include intraoperative frozen section, others have advocated the use of frozen section routinely in TLM. According to these reports, intraoperative frozen section might improve diagnostic accuracy and in some cases avoid a second procedure or the need for adjuvant radiation (22).

Our study, along with the works of other authors, shows that SSTLM can be an acceptable treatment for early glottic cancer. We argue that this management should not be limited to superficial lesions, but can also be applied in cases of infiltrative early glottic tumors or involving the anterior commissure, as was previously described by Perettei et al. and Manola et al. $(2,9)$.

Voice evaluation demonstrated good functional outcomes with better results in cordectomies type I and II than III. With regard to patients in other studies, with early glottic cancer who were treated by TLM after initial biopsies, voice evaluation presented equal to better voice outcomes, indicating functional benefit in cases of SSTLM (23-25).

SSTLM can also spare the burden of a second operation, which include physician, operating room and hospitalization costs, as well as patients' loss of income. If resection of the tumor has been completed at first operation, additional expenses owing to further procedures are saved.

\section{REFERENCES}

1. Zeitels M. Premalignant epithelium and microinvasive cancer of the vocal fold: the evolution of phonomicrosurgical management. Laryngoscope (1995) 105:1-51. doi: 10.1288/00005537-19950300100001

2. Peretti G, Nicolai P, Piazza C, Redaelli De Zinis LO, Valentini S, Antonelli AR. Oncological results of endoscopic resections of Tis and T1 glottic carcinomas by carbon dioxide laser. Ann Otol Rhinol Laryngol. (2001) 110:820-6 doi: 10.1177/0003489401110 00904

3. Nassif R, Loughrab S, Moyes C, Mackenzie K. Negative pathology following endoscopic resection of T1a squamous carcinoma of the glottis. J Laryngol Otol. (2005) 119:592-4 doi: 10.1258/0022215054516322

4. Zapater E, Hernandez R, Reboll R, Perez A, Alba JR, Basterra J. Pathological examination of cordectomy specimens: analysis of negative resection. Auris Nasus Larynx. (2009) 36:321-5 doi: 10.1016/j.anl.2008. 07.007

5. Blakeslee D, Vaughn CW, Shapshay SM, Simpson GT, Strong MS. Excisional biopsy in the selective management of T1 glottic cancer: a three-year follow-up study. Laryngoscope (1984) 94:488-94 doi: 10.1288/00005537-198404000-00012

6. Ossoff RH, Sisson GA, Shapshay SM. Endoscopic management of selected early vocal cord carcinoma. Ann Otol Rhinol Laryngol. (1985) 94:560-4. doi: 10.1177/000348948509400607

7. Peretti G, Cappiello J, Nicolai P, Smussi C, Antonelli AR. Endoscopic laser excisional biopsy for selected glottic carcinomas. Laryngoscope (1994) 104:1276-9. doi: 10.1288/00005537-19941000000017

8. Sigston E, de Mones E, Babin E, Hans S, Hartl DM, Clement P, et al. Early-stage glottic cancer: oncological results and margins in laser cordectomy. Arch Otolaryngol Head Neck Surg. (2006) 132:147-52. doi: 10.1001/archotol.132.2.147

9. Manola M1, Moscillo L, Costa G, Barillari U, Lo Sito S, Mastella A, et al. Conservative laser microsurgery for T1 glottic carcinoma. Auris Nasus Larynx. (2008) 35:141-7. doi: 10.1016/j.anl.2007.08.001
In conclusion, SSTLM for early glottic cancer allows a reliable histopathological diagnosis and a high local control rate with favorable cost effectiveness. Moreover, doing so spares the need for further surgery which can include a more extended cordectomy along with a negative impact on voice outcome. A through patient education and counseling of the various treatment options are needed before obtaining an informed consent. A careful pre- and intraoperative evaluation for selecting the appropriate cases for this management is required in order to avoid under- or over- treatment.

\section{AUTHOR CONTRIBUTIONS}

$\mathrm{YH}$ and AM designed the study. YH, DY, SD-S, and SS collected and organized the clinical data and performed the statistical analysis. YH, HS-H, and AM drafted and reviewed the manuscript. SD-S and SS revised it critically for important intellectual content. All the authors read and approved the final manuscript.

\section{FUNDING}

This research received no specific grant from any funding agency in the public, commercial, or not-for-profit sectors.

10. Remacle M, Eckel H, Antonelli A, Brasnu D, Chevalier D, Friedrich G, et al. Endoscopic cordectomy a proposal for classification by the Working Committee European Laryngological Society. Eur Arch Otorhinolaryngol. (2002) 257:227-31. doi: 10.1007/s004050050228

11. Hogikyan ND, Sethuraman G. Validation of an instrument to measure voicerelated quality of life (V-RQOL). J Voice (1999) 13:557-69.

12. A Hirano M. Clinical Examination of Voice: Volume 5 of Disorders of Human Communication. Berlin: Springer London, Limited (1981).

13. Greene FL, Page DL, Fleming ID, Fritz A, Balch C, Haller DG, et al. ALCC Cancer Staging Manual, 6th edn. New York, NY: Springer (2002).

14. Zeitels SM. Premalignant epithelium and microinvasive cancer of the vocal fold: the evolution of phonomicrosurgical management. Laryngoscope (1995) 67:1-51.

15. Zeitels SM. Phonomicrosurgical treatment of early glottic cancer and carcinoma in situ. Am J Surg. (1996) 172:704-9.

16. Delsupehe KG, Zink I, Lejaegere M, Bastian RW. Voice quality after narrow-margin laser cordectomy compared with laryngeal irradiation. Otolaryngol Head Neck Surg. (1999) 121:528-33. doi: 10.1016/S0194-5998(99) 70051-3

17. Davis RK, Kelly SM, Parkin JL, Stevens MH, Johnson LP. Selective management of early glottic cancer. Laryngoscope (1990) 100:1306-9. doi: 10.1288/00005537-199012000-00011

18. M Lucioni, G Marioni, A bertolin, L Giacomelli, G Rizzotto. Glottic laser surgery: outcomes according to 2007 RLS Classification. Eur Arch Otorhinolaryngol. (2011) 268:1771-8. doi: 10.1007/s00405-0111695-7

19. Peretti G, Piazza C, Berlucchi M, Cappiello J, Giudice M, Nicolai P. Pre- and intraoperative assessment of mid-cord erythroleukoplakias: a prospective study on 52 patients. Eur Arch Otorhinolaryngol. (2003) 260:5258. doi: 10.1007/s00405-003-0584-0

20. Hirano M, Bless DM. Videostroboscopic Examination of the Larynx. California: Singular Publishing (1993).

21. Lai A, Zeitels SM. Endoscopic treatment of early glottic carcinoma. Curr Opin Otolaryngol Head Neck Surg. (1996) 4:126-33 doi: 10.1097/00020840-199604000-00011 
22. Remacle M, Matar N, Delos M, Nollevaux MC, Jamart J, Lawson G. Is frozen section reliable in transoral $\mathrm{CO}(2)$ laser-assisted cordectomies? Eur Arch Otorhinolaryngol. (2010) 267:397-400. doi: 10.1007/s00405-0091101-x

23. Keilmann A, Napiontek U, Engel C, Nakarat T, Schneider A, Mann W. Long-term functional outcome after unilateral cordectomy. ORL J Otorhinolaryngol Relat Spec. (2010) 73:38-46. doi: 10.1159/0003 22592

24. Chu PY, Hsu YB, Lee TL, Fu S, Wang LM, Kao YC. Longitudinal analysis of voice quality in patients with early glottic cancer after transoral laser microsurgery. Head Neck (2012) 34:1294-8. doi: 10.1002/hed. 21914

25. Lombardo N, Aragona T, Alsayyad S, Pelaia G, Terracciano R, Savino R. Objective and self-evaluation voice analysis after transoral laser cordectomy and radiotherapy in T1a-T1b glottic cancer. Lasers Med Sci. (2018) 33:141-7. doi: 10.1007/s10103-017-2361-0

Conflict of Interest Statement: The authors declare that the research was conducted in the absence of any commercial or financial relationships that could be construed as a potential conflict of interest.

Copyright (c) 2018 Hamzany, Shoffel-Havakuk, Devons-Sberro, Shteinberg, Yaniv and Mizrachi. This is an open-access article distributed under the terms of the Creative Commons Attribution License (CC BY). The use, distribution or reproduction in other forums is permitted, provided the original author(s) and the copyright owner(s) are credited and that the original publication in this journal is cited, in accordance with accepted academic practice. No use, distribution or reproduction is permitted which does not comply with these terms. 
OPEN ACCESS

Edited by:

Remco De Bree,

University Medical Center Utrecht,

Netherlands

Reviewed by:

Edgar K. Selzer,

Medical University of Vienna, Austria

Sebastien Vergez,

Institut universitaire du Cancer de

Toulouse Oncopole, France

*Correspondence: Cesare Piazza

cesare.piazza@istitutotumori.mi.it,

ceceplaza@libero.it

Specialty section:

This article was submitted

to Head and Neck Cancer,

a section of the journal

Frontiers in Oncology

Received: 14 July 2017

Accepted: 29 September 2017

Published: 16 October 2017

Citation:

Fiz I, Mazzola F, Fiz F, Marchi F, Filauro M, Paderno A, Parrinello G, Piazza C and Peretti G (2017) Impact of Close and Positive Margins in

Transoral Laser Microsurgery for Tis-T2 Glottic Cancer.

Front. Oncol. 7:245.

doi: 10.3389/fonc.2017.00245

\section{Impact of Close and Positive Margins in Transoral Laser Microsurgery for Tis-T2 Glottic Cancer}

\author{
Ivana Fiz ${ }^{1,2}$, Francesco Mazzola ${ }^{1}$, Francesco Fiz ${ }^{3,4}$, Filippo Marchi', Marta Filauro', \\ Alberto Paderno ${ }^{5}$, Giampiero Parrinello ${ }^{1}$, Cesare Piazza ${ }^{6 *}$ and Giorgio Peretti ${ }^{1}$ \\ ${ }^{1}$ Department of Otorhinolaryngology - Head and Neck Surgery, University of Genoa, Genoa, Italy, ${ }^{2}$ Department of \\ Otorhinolaryngology - Head and Neck Surgery, Katharinenhospital, Stuttgart, Germany, ${ }^{3}$ Nuclear Medicine Unit, Department \\ of Radiology, Uni-Klinikum Tuebingen, Tuebingen, Germany, ${ }^{4}$ Department of Internal Medicine, University of Genoa, Genoa, \\ Italy, ${ }^{5}$ Department of Otorhinolaryngology - Head and Neck Surgery, University of Brescia, Brescia, Italy, ${ }^{6}$ Department of \\ Otorhinolaryngology - Head and Neck Surgery, Fondazione IRCCS - National Cancer Institute of Milan, University of Milan, \\ Milan, Italy
}

Introduction: Transoral laser microsurgery (TLM) represents one of the most effective treatment strategies for Tis-T2 glottic squamous cell carcinomas (SCC). The prognostic influence of close/positive margins is still debated, and the role of narrow band imaging (NBI) in their intraoperative definition is still to be validated on large cohort of patients. This study analyzed the influence of margin status on recurrence-free survival (RFS) and disease-specific survival (DSS).

Methods: We retrospectively studied 507 cases of pTis-T1b (Group A) and 127 cases of pT2 (Group B) glottic SCC. We identified the following margin status: negative $(n=232)$, close superficial ( $n=79)$, close deep (CD) $(n=35)$, positive single superficial $(n=146)$, positive multiple superficial $(n=94)$, and positive deep $(n=48)$ and analyzed their impact on RFS and DSS. Close margins were defined by tumor-margin distance $<1 \mathrm{~mm}$. PreTLM margins were defined by white light in 323 patients, whereas NBI was employed in 311 patients.

Results: In Group A, DSS and RFS were reduced in positive multiple superficial and positive deep margins (DSS $=96.1$ and 97\%, both $p<0.05$; RFS $=72 \%, p<0.001$ and $75.8 \%, p<0.01$ ). In Group B, DSS was reduced in positive multiple superficial margins (82.4\%, $p<0.05)$. RFS was reduced in positive single superficial, positive multiple superficial, and positive deep margins $(62.5,41.2$, and $53.3 \%, p<0.01)$. In the entire population, RFS was reduced in CD margins $(77.1 \%, p<0.05)$. Use of NBI led to improvement in RFS and DSS.

Conclusion: The study indicates that close and positive single superficial margins do not affect DSS. By contrast, all types of margin positivity predict the occurrence of relapses, albeit with different likelihood, depending on stage/margin type. CD margins should be considered as a single risk factor. Use of NBI granted better intraoperative margins definition.

Keywords: laryngeal cancer, early glottic cancer, transoral laser microsurgery, $\mathrm{CO}_{2}$ laser, surgical margins, endoscopy, recurrence-free survival, disease-specific survival 


\section{INTRODUCTION}

Early glottic squamous cell carcinoma (SCC) has an excellent local control rate (LCR) independently of the treatment choice. In particular, for T1 category, LCR is reported to range from 86 to $95 \%$ for transoral laser microsurgery (TLM) and from 85 to $94 \%$ for radiotherapy (RT) (1-4). In T2 lesions, RT shows a slightly worse LCR, ranging from 67 to $76 \%$, with respect to TLM (76-89\%) (1-8). However, a large meta-analysis of 11 studies, involving 1,135 patients, failed to identify any difference in LCR of patients with early glottic SCC receiving TLM or RT, even though overall costs were lower with TLM (7). Despite the above reported similar LCR, present evidence suggests that RT may perform worse than TLM in terms of disease-free survival (9); moreover, the radiation therapy approach seems to entail a higher risk for subsequent total laryngectomy in comparison with $\operatorname{TLM}(10)$.

On the other hand, the choice of TLM implies the assessment of a series of patient-dependent and technique-related factors, to obtain the most favorable therapeutic outcome (11-14). One of the most influencing factors in TLM is definitively represented by surgical margins status after surgery. This issue is complicated by the lack of a homogeneous definition of negative, close, and positive margins. In fact, most authors define a margin as "negative" when the tumor-margin distance is $>1 \mathrm{~mm}$, "close" when the distance is $<1 \mathrm{~mm}$, and "positive" in presence of tumor at the surgical edge. However, management of patients with close and positive margins is still controversial (15-18). Specific indications for surgical re-treatment (by TLM or open-neck approaches) or complementary RT and the impact of such adjuvant treatments on recurrence-free survival (RFS) and disease-specific survival (DSS) are not yet clearly defined.

In light of this, we retrospectively analyzed a large homogeneous cohort of patients affected by early glottic SCC treated by TLM, focusing our attention on the impact of close and positive surgical margins on RFS and DSS, and on the possibility to reduce their rates by using new biologic endoscopy tools such as narrow band imaging (NBI).

\section{MATERIALS AND METHODS}

We retrospectively analyzed data from a series of 634 untreated patients (560 males, 74 females; mean age, $64.1 \pm 10.4$ years; age range, 30-88) affected by Tis-T2 glottic SCC who underwent TLM from January 2000 to March 2014 at the Departments of Otorhinolaryngology-Head and Neck Surgery, University of Genoa and Brescia, Italy (Table 1). All patients signed a written informed consent, which was reviewed and approved by the local Ethics Committees and including the use of anonymized patient data for research purposes.

The tumors were intraoperatively assessed by both $0^{\circ}$ and $70^{\circ}$ rigid telescopes (Olympus Medical System Corporation, Tokyo, Japan and Karl Storz, Tuttlingen, Germany), increasing the accuracy of neoplastic superficial spreading evaluation (19). In all patients since January 2008, preoperative videolaryngoscopy was combined with high definition television (HDTV)-NBI (Olympus Medical System Corporation, Tokyo, Japan) (20). In selected
TABLE 1 | Demographic characteristics, patients' stratification by pTNM, margin status, and types of cordectomy performed.

\begin{tabular}{|c|c|c|c|}
\hline Variables & Entire cohort & Group A & Group B \\
\hline Number of patients & 634 & 507 & 127 \\
\hline Age & $64.1 \pm 10.4$ & $64.1 \pm 10.2$ & $64.3 \pm 11.2$ \\
\hline Male/female & $560 / 74$ & $467 / 40$ & $93 / 34$ \\
\hline \multicolumn{4}{|l|}{ T category } \\
\hline pTis & 102 & 102 & - \\
\hline pT1a & 316 & 316 & - \\
\hline pT1b & 89 & 89 & - \\
\hline pT2 & 127 & - & 127 \\
\hline \multicolumn{4}{|c|}{ Margin status, no. (\%) } \\
\hline NEG & $231(36.4)$ & 199 (39.2) & 32 (25.2) \\
\hline CS & $79(12.4)$ & $58(11.4)$ & $21(16.5)$ \\
\hline $\mathrm{CD}$ & $35(5.5)$ & $25(4.9)$ & $10(7.9)$ \\
\hline SS & $146(23)$ & $114(22.5)$ & $32(25.2)$ \\
\hline MS & $94(14.8)$ & $77(15.2)$ & $14(13.4)$ \\
\hline DEEP & $48(7.5)$ & $33(6.5)$ & $15(11.8)$ \\
\hline \multicolumn{4}{|c|}{ Type of cordectomy } \\
\hline I & 48 & 48 & - \\
\hline$\|$ & 275 & 262 & - \\
\hline III & 122 & 111 & - \\
\hline IV & 40 & 27 & 23 \\
\hline V & 141 & 56 & 99 \\
\hline VI & 8 & 3 & 5 \\
\hline
\end{tabular}

NEG, negative margin; CS, close superficial; CD, close deep; SS, positive single superficial; MS, positive multiple superficial; DEEP, positive deep margins.

cases, CT or MR was carried out to evaluate anterior commissure, visceral spaces, and laryngeal framework involvement.

Adequate laryngeal exposure in microlaryngoscopy was obtained by different laryngoscopes comprising Sataloff (Microfrance ${ }^{\circledR} \mathrm{iXomed}$, Saint Aubin Le Monial, France), Dedo, and Dedo-Ossoff (Pilling, Philadelphia, PA, USA). The lasers used were the Sharplan $1055 \mathrm{~S}$ (Sharplan, Tel Aviv, Israel) from 2000 to 2004, and the UltraPulse/Surgitouch $\mathrm{CO}_{2}$ laser (Lumenis, Yokneam, Israel) from 2004 to 2014.

Patients were treated by six different types of cordectomies according to the European Laryngological Society classification (Table 1), using "en bloc" or "multi bloc" approaches, in relation to the tumor site, size, category, depth of infiltration, and laryngeal exposure (21-23). Frozen sections were not routinely performed. Extra-surgical margins, when deemed necessary, were taken from the surgical bed after resection of larger lesions and sent separately for definitive histopathologic examination.

On the basis of histopathological reports, the entire cohort was divided in two groups: Group A included 507 (102 pTis, 316 pT1a, and 89 pT1b) subjects and Group B 127 (all pT2) patients (Table 1), staged according to the seventh TNM classification by the American Joint Committee on Cancer (17).

We defined surgical margins as follows: negative (distance tumor-margin $>1 \mathrm{~mm}$ ), close (distance tumor-margin between 0 and $1 \mathrm{~mm}$ ), and positive (presence of at least carcinoma in situ at the surgical margin). In case of extra-surgical margins taken at the end of procedure, these were considered as the definitive surgical margins.

Median follow-up for the entire cohort of patients was 60 months (range, 12-176): those staged as pTis-pT1b with negative margins were followed by videolaryngoscopy every 2 months in the first year, every 3 months in the second, every 4 months in 
the third, every 6 months in the fourth, and once a year afterward. In patients staged as pT2, MR or CT was added every 6 months for the first 2 years of follow-up even if the endoscopy was negative.

In case of close margins and/or single positive superficial margin, a monthly follow-up and, where indicated, periodic imaging were performed. In case of positive multiple superficial or deep margins, intraoperative recording was reviewed and, after multidisciplinary discussion, an adjuvant treatment (transoral re-resection, open partial laryngectomy, or adjuvant RT) was proposed to the patient. Whenever this option was refused by the patient or deemed too risky for his/her general conditions, a strict watch-and-see policy was followed.

\section{Statistical Analysis}

The SPSS program (SPSS, v. 21.0, IBM, Armonk, NY, USA) was used for statistical analysis. Five-year survival curves were plotted using the Kaplan-Meier method; pairwise over strata log-rank test was used to detect survival differences between groups. Analysis was first performed for the entire patient population and then separately for Groups A and B.

The entry point was the date of laser cordectomy. Differences between survival curves were assessed using log-rank test for margin status variables. Six different margin statuses were considered for the Kaplan-Meier survival curve: negative (NEG), close superficial (CS), close deep (CD), positive single superficial (SS), positive multiple superficial (MS), and positive deep margins (DEEP).

The first studied outcome was DSS, with the end point being patient's death or last follow-up (censored observations, shown as "+" symbol along the survival line). Patients who died of unrelated causes were excluded from the analysis. The second outcome was RFS, with the end point set at the date of recurrence or at the last available visit (censored observations). Organ preservation (OP) was the third measured outcome, with the end point set at the date of total laryngectomy or at last follow-up (censored observations).

Influence of the routine use of HDTV-NBI in defining superficial resection margins and its impact on RFS, DSS, and OP were also investigated by comparing recurrence rates, disease-specific lethal events, and need for total laryngectomy between patients treated before NBI implementation (pre-NBI group, 2000-2007) vs. those operated on thereafter (NBI group, 2008-2014). This analysis was carried out for the entire group of patients, and for Group A, Group B, and pT1a lesions separately. Differences in number of events between the pre-NBI and NBI groups were assessed using chi-squared or Fisher's exact tests, as appropriate.

Moreover, relative risk of relapse related to margin positivity, age, tumor stage, use of HDTV-NBI for margin definition, and additional treatment after the intervention was tested by using a Cox multivariate model with backward logistic regression. For T2 patients, influence of tumor pattern of spread (i.e., transcommissural, supraglottic, and subglottic extension, as well as muscle infiltration) was further tested with the same model.

\section{RESULTS}

Among all patients, 288 (45.4\%) had positive margins. In particular, 146 (23\%) had positive single superficial margins, 94 (14.8\%) positive multiple superficial margins, and 48 (7.5\%) positive deep margins. One hundred fourteen patients (18\%) had close margins, among which 79 (12.5\%) CS and 35 (5.5\%) CD margins (Table 1).

\section{Impact of Margin Status on DSS}

In the entire cohort, patients with multiple positive superficial margins (MS) had reduced DSS compared with those with negative ones ( 93.6 vs. $100 \%, p=0.005$, Figure 1). In details, in Group A, both positive multiple superficial (MS) and positive deep margins (DEEP) status were related to a slight, yet significant, reduction in DSS (96.1 and 97\%, respectively vs. 100\%, $p<0.05$, Figure 2), whereas in Group B all events were observed in patients affected by positive multiple superficial margins (MS) compared with those with negative ones ( 82.4 vs. $100 \%, p=0.019$, Figure 2).

\section{Impact of Margin Status on RFS}

Overall, RFS was affected by margin status, as $88.2 \%$ of patients with negative and close margins were recurrence free at their last follow-up, compared with $73.3 \%$ of patients with positive margins $(p<0.001)$. All subtypes of margins positivity predicted recurrence: RFS for SS, MS, and DEEP margins was 78.8\% $(p<0.01)$, $67 \%(p<0.001)$, and 68.8\% ( $p<0.001)$, respectively (Figure 3). At multivariate Cox regression, relative risk of recurrence for this three margin infiltration patterns was $2.1,3.7$, and 3.4, respectively ( $p<0.01$, Table 2$)$.

In Group A, patients with positive margins had a 78.6\% RFS, which was reduced in comparison with patients with both negative and close margins together $(89.4 \%, p<0.05)$. Overall, the difference between negative and all positive margins was significant ( $p=0.002)$; in particular, RFS for SS, MS, and DEEP margins was $83.3 \%(p=\mathrm{NS}), 72.7 \%(p<0.001)$, and $75.8 \%(p<0.01)$, respectively. Anterior commissure involvement did not associate with lower RFS (93.5\% in tumors reaching the anterior commissure vs. $93.6 \%$ in those not involving this subsite, $p=\mathrm{NS}$ ).

In Group B, if patients with negative and close margins were considered as a single group, RFS was $82 \%$. All subtypes of margin positivity were indicative of worse RFS, which decreased to $54.7 \%$ in patients whose resection margins were positive $(p<0.01)$. Specifically, the percentage of patients with no evidence of recurrence throughout follow-up was $62.5 \%(p<0.05)$, $41.2 \%(p<0.001)$, and $53.3 \%(p=0.012)$ for SS, MS, and DEEP margin positivity, respectively. Different tumor extension (transcommissural, supraglottic, subglottic, or massive vocal muscle infiltration) did not show any differences in RFS (68.2, 67.8, 70.7, and $74.4 \%$ respectively, $p=$ NS) (Figure 4 ).

\section{Impact of Close Margins}

Close margins did not affect DSS. In the entire population, patients with CS margins did not show a significant decrease in RFS $(81 \%, p=N S)$; conversely, the presence of CD margins was related to a significantly increased number of relapses with an RFS of $77.1 \%$ and a relative risk of 2.6 (Table $2, p<0.05$ ).

\section{Organ Preservation}

Deep margin infiltration predicted a worse OP in Group A. In fact, no patients with negative margins had to be treated by 


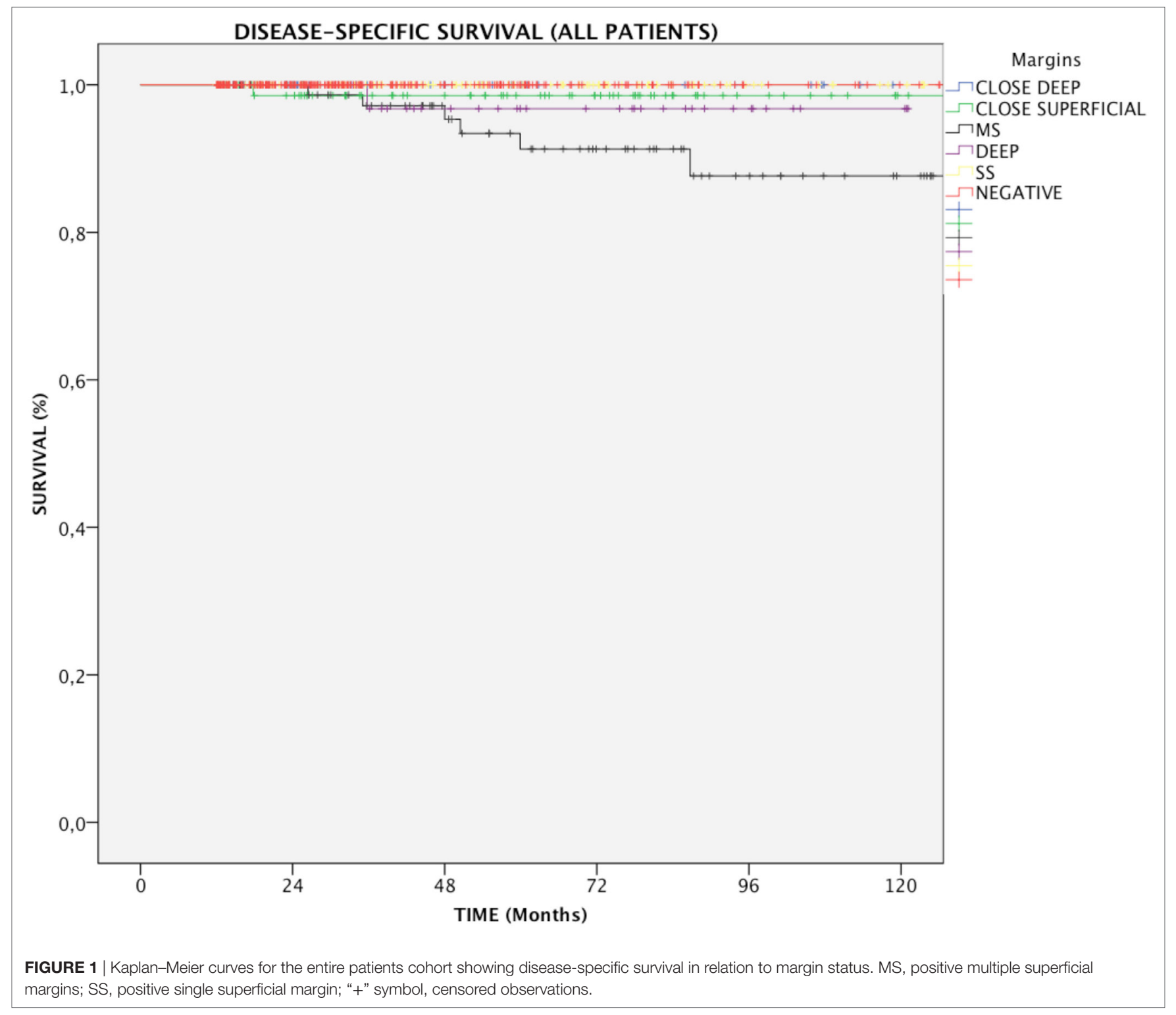

total laryngectomy, while $6.1 \%$ of those with positivity of the deep margin lost the larynx $(p<0.01)$. No significant difference was observed in Group B. Detailed figures regarding RFS, DSS, and OP are shown in Table 3.

\section{Impact of Additional Treatment}

Twelve of 94 patients (12.8\%) with positive MS margins underwent additional treatment (11 TLM and 1 adjuvant RT) due to the presence of adjunctive histopathologic risk factors such as high-grade lesions, evidence of perineural spread and/or vascular embolization. The presence of such selection bias could explain why these patients had worse RFS (50\%) than those who did not undergo re-treatment $(69.5 \%, p=0.03)$.

Fifteen of 48 patients (31.2\%) with positive deep margins underwent additional treatment (9 TLM and 6 adjuvant RT). Among these, additional treatment showed slight, though non-significant improvement of RFS (73.3\%) compared with those who received no further treatment $(66.7 \%, p=\mathrm{NS})$. Detailed figures regarding additional treatments are available in Table 4.

\section{Impact of HDTV-NBI on Margins Evaluation, RFS, DSS, and OP}

In all patients, intraoperative use of HDTV-NBI allowed improved evaluation of surgical margins, with a greater proportion of negative margins (50 vs. $30 \%, p<0.001$ ), reduced number of CS (10 vs. $16 \%, p<0.001$ ), and multiple superficial margins (15 vs. $17 \%, p=0.03$ ). Improvement of the rate of negative margins was also observable in Group A (45 vs. $34 \%, p<0.001$ ) and in pT1a patients ( 44 vs. $31 \%, p<0.001$ ). Moreover, use of HDTV-NBI reduced the postoperative findings of CS ( 8 vs. $15 \%, p<0.001$ ) and multiple superficial positive margins ( 13 vs. $18 \%, p=0.01$ ) in both Group A and pT1a patients (9 vs. $18 \%, p<0.001 ; 10$ vs. $17 \%, p<0.001$, respectively). 

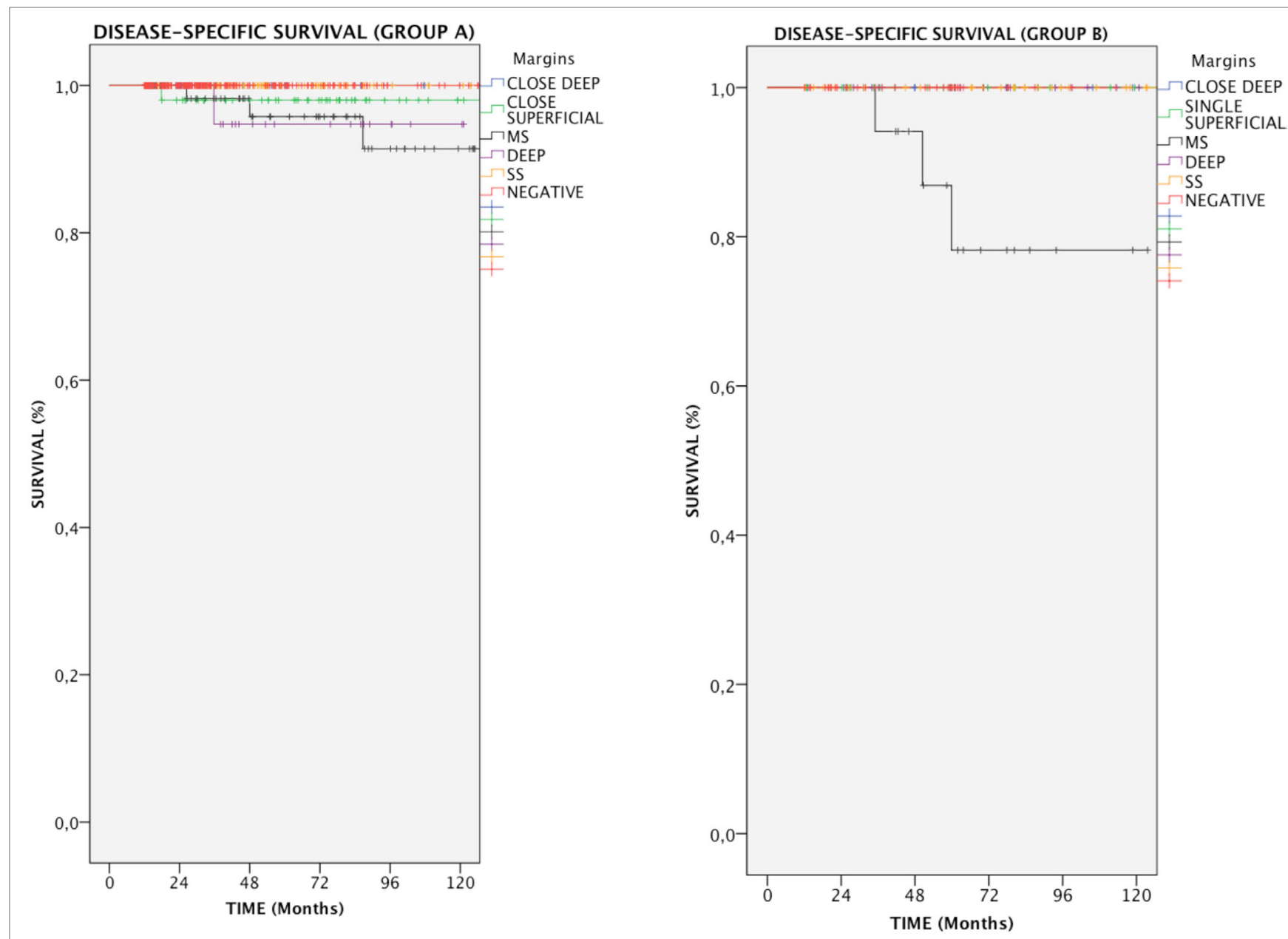

FIGURE 2 | Kaplan-Meier curves showing disease-specific survival in relation to margin status for each group of patients. MS, positive multiple superficial margins; SS, positive single superficial margin; "+" symbol, censored observations.

In Group B, use of NBI significantly improved the relative number of negative margins ( 46 vs. $15 \%, p<0.001$ ), while CS (24 vs. $29 \%, p<0.001$ ) and single superficial positive margins (13 vs. $21 \%, p<0.001)$ were significantly reduced. As expected, NBI did not affect the rate of positive or CD margins.

In all patients, use of NBI significantly improved RFS (83.9 vs. $78.9 \% p<0.05)$. Such improvement was particularly appreciable in negative ( 94.1 vs. $89.6 \%, p<0.01)$ and multiple superficial margins ( 75 vs. $61.1 \%, p<0.001$ ). Relative risk, as calculated by multivariate Cox regression, was reduced in patients treated with the help of HDTV-NBI $(0.61, p<0.05)$ (Table 2).

Similarly, NBI was beneficial in negative and MS margins when Group A $(p<0.001)$ or pT1a lesions $(p=0.01)$ were considered. No difference in RFS was detected in Group B. Use of HDTV-NBI also improved DSS in patients with MS margins (Table 5).

\section{Impact of Additional Factors on Survival}

At multivariate Cox regression analysis, the main factor having an impact on RFS was the surgical margin status $(p<0.001)$
(Table 2). All margin positivity affected RFS; however, in accordance to what demonstrated by the Kaplan-Meier model, multiple superficial and deep margin infiltrations were associated with the highest risk increase (Table 2). Other factors showing a significant incidence on RFS were T category $(p<0.01)$, with T1b and T2 lesions showing increased risk $(p<0.05)$, and use of HDTV-NBI, exerting a protective effect against future recurrences $(p<0.05)$. Age, adjuvant treatment and, for T2 tumors, presence of specific patterns of spread such as transcommissural, supraglottic, subglottic, or massive muscle infiltration, did not affect the risk of developing relapse.

\section{DISCUSSION}

Transoral laser microsurgery is a surgical approach that has emerged as a viable alternative to open-neck approaches and RT as it allows sound oncological results, while preserving organ function and ensuing high salvage rates in case of persistent/ recurrent or secondary laryngeal tumors (1-8). Moreover, its favorable cost-effectiveness ratio profile could be helpful in 


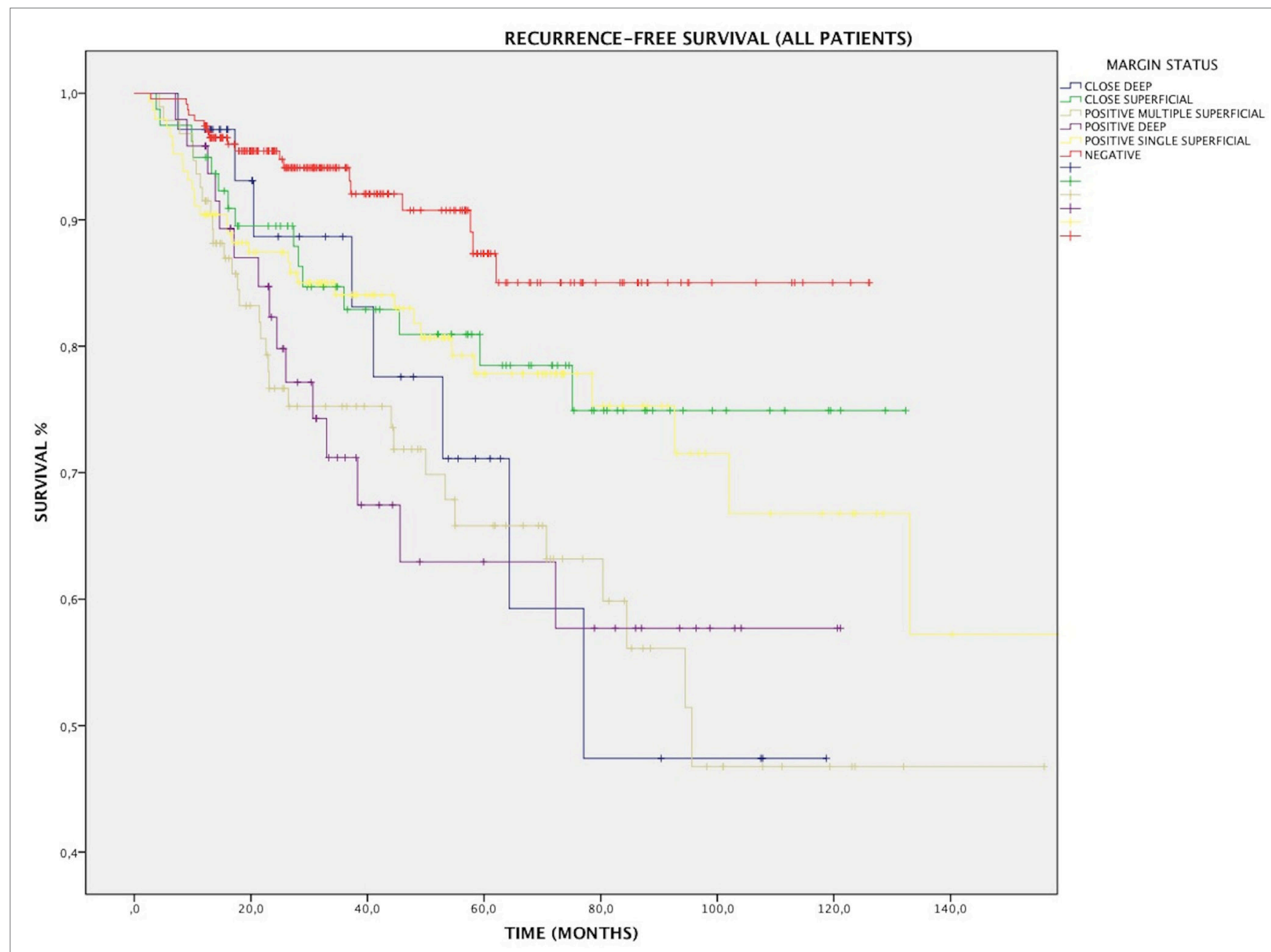

FIGURE 3 | Kaplan-Meier curves for the entire patients cohort showing recurrence-free survival in relation to margin status. MS, positive multiple superficial margins SS, positive single superficial margin; "+" symbol, censored observations.

TABLE 2 | Cox regression analysis of factors affecting recurrence-free survival.

\begin{tabular}{|c|c|c|c|c|c|}
\hline & \multirow[t]{2}{*}{$B$} & \multirow[t]{2}{*}{ Sig. } & \multirow[t]{2}{*}{$\operatorname{Exp}(B)$} & \multicolumn{2}{|c|}{$\begin{array}{l}\text { Cl } 95.0 \% \text { for } \\
\quad \exp (B)\end{array}$} \\
\hline & & & & Lower & Upper \\
\hline \multicolumn{6}{|l|}{ All patients } \\
\hline Margins & & $<0.001$ & 3.703 & 2.055 & 6.672 \\
\hline Age & & NS & 1.002 & 0.984 & 1.021 \\
\hline Additional treatment & & NS & 1.113 & 0.586 & 2.113 \\
\hline TNM stage & 0.371 & 0.002 & 1.853 & 0.975 & 3.522 \\
\hline Narrow band imaging & -0.491 & 0.016 & 0.612 & 0.411 & 0.911 \\
\hline \multicolumn{6}{|l|}{ T2 patients } \\
\hline $\begin{array}{l}\text { Transcommissural } \\
\text { infiltration }\end{array}$ & 0.06 & NS & 1.062 & 0.452 & 2.496 \\
\hline Muscle infiltration & 0.667 & NS & 1.949 & 0.939 & 4.043 \\
\hline Hypoglottic infiltration & -0.228 & NS & 0.796 & 0.363 & 1.746 \\
\hline Supraglottic infiltration & -0.071 & NS & 0.932 & 0.419 & 2.071 \\
\hline
\end{tabular}

$B$, beta coefficient; Wald, Wald statistic; df, degrees of freedom; Exp (B), hazard ratio or relative risk; $\mathrm{Cl}$ 95.0\% for $\exp (B)$, confidence interval 95.0\%; NS, non-significant. containing ever-growing healthcare costs (7). This type of surgery is mostly applied in early-stage disease and is characterized by a very narrow-margin approach, which makes the process of performing a safe and clean resection challenging. Moreover, the use of laser invariably leads to tissue dehydration and consequent margin shrinkage, which further reduces the ability to obtain widely negative surgical margins at histopathological examination (23). These two aspects force the surgeon to put significant effort in balancing between the best oncological and functional outcomes in each patient.

Actually, one of the most important prognostic factors in TLM remains the status of surgical margins $(11,14,16)$. In the present series, we confirmed the feasibility and oncological soundness of this technique, as demonstrated by the high RFS and DSS rates, which favorably compare to those reported in the literature $(1,5,6)$. Moreover, it should always be kept in mind that, in TLM for early glottic SCC, RFS does not necessarily have a significant impact on DSS, LCR, or OP, due to the high percentage of recurrences that are salvageable by further 

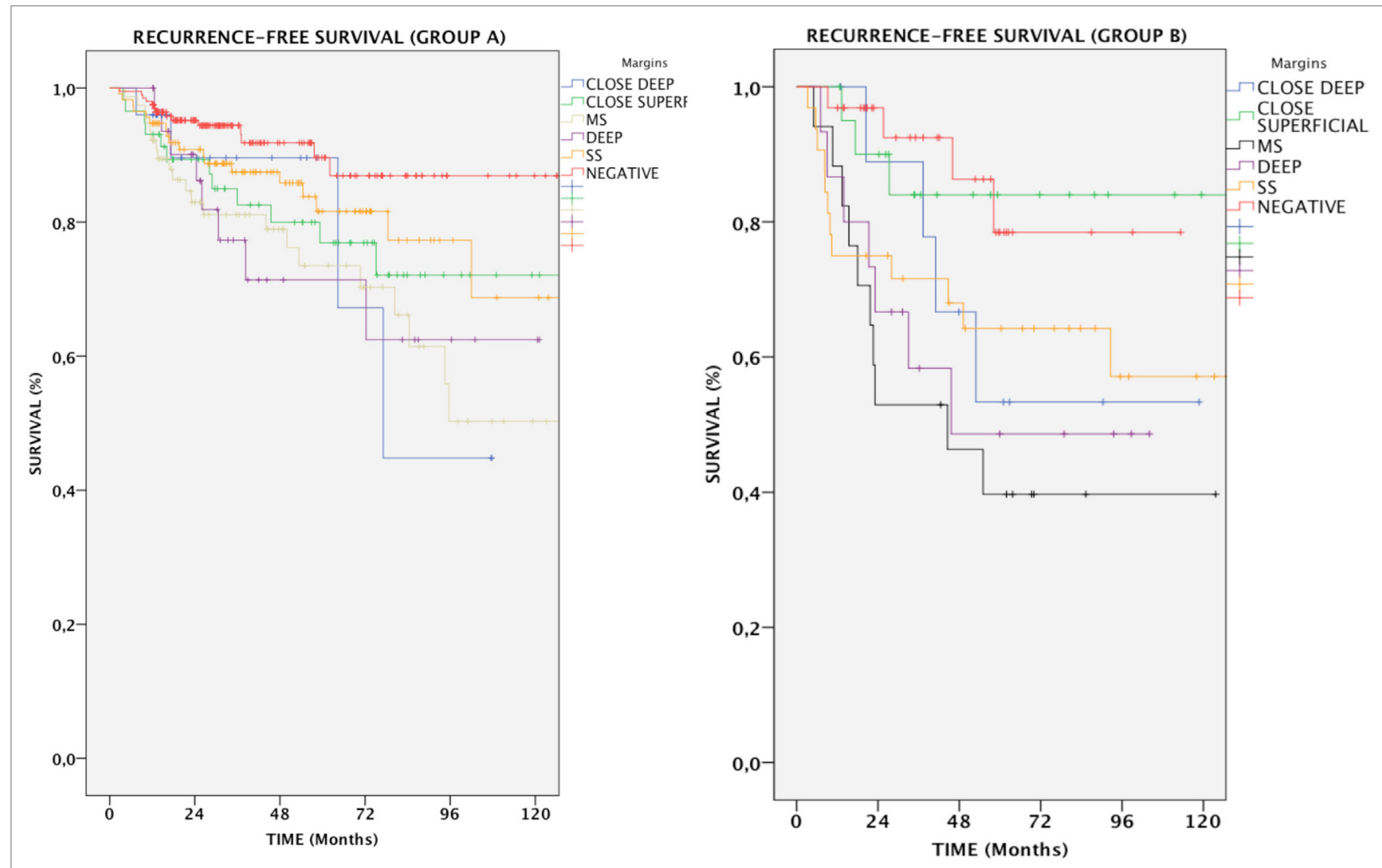

FIGURE 4 | Kaplan-Meier curves showing recurrence-free survival in relation to margin status for each group of patients. MS, positive multiple superficial margins; SS, positive single superficial margin; "+" symbol, censored observations.

TABLE 3 | Five-year disease-specific survival (DSS), recurrence-free survival (RFS), and organ preservation (OP) for the entire cohort, and for Groups A and B.

\begin{tabular}{|c|c|c|c|}
\hline & DSS (\%) & RFS (\%) & OP (\%) \\
\hline \multicolumn{4}{|c|}{ All patients } \\
\hline NEG & 100 & 92.2 & 98.7 \\
\hline CS & 98.7 & 81 & 98.7 \\
\hline CD & 100 & $77.1^{*}$ & $91.4^{*}$ \\
\hline SS & 100 & $78^{\star \star}$ & 97.3 \\
\hline MS & $93.6^{\star *}$ & $67^{\star \star \star}$ & 95.7 \\
\hline DEEP & 97.9 & $68^{\star \star \star}$ & 95.8 \\
\hline \multicolumn{4}{|c|}{ Group A } \\
\hline NEG & 100 & 93 & 100 \\
\hline CS & 98.3 & $79.3^{*}$ & 100 \\
\hline CD & 100 & 84 & $96^{\star \star}$ \\
\hline SS & 100 & 83.3 & 99.1 \\
\hline MS & $96.1^{*}$ & $72^{\star \star \star}$ & 98.7 \\
\hline DEEP & $97^{\star}$ & $75.8^{\star \star}$ & $93.9^{\star \star}$ \\
\hline \multicolumn{4}{|c|}{ Group B } \\
\hline NEG & 100 & 87.5 & 90.6 \\
\hline CS & 100 & 85.7 & 95.2 \\
\hline $\mathrm{CD}$ & 100 & 60 & 80 \\
\hline SS & 100 & $62.5^{\star}$ & 90.6 \\
\hline MS & $82.4^{*}$ & $41.2^{\star \star \star}$ & 82.4 \\
\hline DEEP & 100 & $53.3^{\star}$ & 100 \\
\hline
\end{tabular}

p-Values for comparisons between negative margins and all kinds of positive/close margins are indicated as $\left(^{*}\right)$ when $<0.05$, $\left(^{* *}\right)$ when $<0.01$, and $\left(^{* * *}\right)$ when $<0.001$. NEG, negative margin; CS, close superficial; CD, close deep; SS, positive single superficial; MS, positive multiple superficial; DEEP, positive deep margins.
TABLE 4 | Impact of additional treatments in all patients on RFS.

\begin{tabular}{lcccc}
\hline & $\begin{array}{c}\text { Positive multiple } \\
\text { superficial } \\
\text { margins }\end{array}$ & $\begin{array}{c}\text { Positive deep } \\
\text { margins }\end{array}$ \\
\hline Patients & 94 & RFS & 48 & RFS \\
Without additional treatment & 82 & $69.5 \%$ & 33 & $66.7 \%$ \\
With additional treatment & 12 & $50 \%$ & 15 & $73.3 \%$ \\
$p$ & & 0.03 & & $\mathrm{NS}$
\end{tabular}

RFS, recurrence-free survival; NS, non-significant.

conservative treatment options (1). In fact, our data show that even when surgery is executed in the best-case scenario and the surgical margins are completely clean, recurrence is still possible and may affect up to one-tenth of all patients during follow-up. This figure shows a slight variation according to tumor category, which is, however, of minor significance. Use of HDTV-NBI further improved this outcome, as only one in 20 patients with negative margins suffered from disease recurrence after introduction of this bio-endoscopic tool in our routine practice. No patient with negative margins died of disease, thus confirming their good prognostic role.

The same line of thinking can be applied to close surgical margins, as no such patient died of disease. However, the surgeon may expect a higher recurrence rate with respect to fully negative 
TABLE 5 | Impact of narrow band imaging (NBI) on 5-year recurrence-free survival (RFS) and disease-specific survival (DSS) for the entire cohort of patients, for Groups A and B, and for pT1a patients.

\begin{tabular}{|c|c|c|c|c|c|c|}
\hline & \multicolumn{2}{|c|}{ Patients (\%) } & \multicolumn{2}{|c|}{ RFS (\%) } & \multicolumn{2}{|c|}{ DSS (\%) } \\
\hline & Pre-NBI & NBI & Pre-NBI & NBI & Pre-NBI & NBI \\
\hline All patients & 323 & 311 & 78.9 & $83.9^{*}$ & 98.8 & 98.7 \\
\hline NEG & $96(30)$ & $136(50)^{\star \star \star}$ & 89.6 & $94.1^{\star \star}$ & 100 & 100 \\
\hline CS & $53(16)$ & $26(10)^{\star \star \star}$ & 90.6 & 73.1 & 100 & 96.2 \\
\hline SS & $78(24)$ & $68(25)$ & 80.8 & 76.5 & 100 & 100 \\
\hline MS & $54(17)$ & $40(15)^{\star}$ & 61.1 & $75^{\star \star \star}$ & 92.6 & $95^{\star}$ \\
\hline Group A (Tis-T1) & 251 & 256 & 83.3 & 87.1 & 99.2 & 98.8 \\
\hline NEG & 85 (34) & $115(45)^{\star \star \star}$ & 89.4 & $95.6^{\star \star \star}$ & 100 & 100 \\
\hline CS & $38(15)$ & $20(8)^{\star \star \star}$ & 89.5 & 75 & 100 & 95 \\
\hline SS & $57(23)$ & $57(22)$ & 94.3 & 79 & 100 & 100 \\
\hline MS & $45(18)$ & $32(13)^{\star \star}$ & 66.7 & $81.2^{\star \star \star}$ & 95.6 & 96.9 \\
\hline T1a & 160 & 157 & 86.3 & 86.5 & 99.4 & 99.4 \\
\hline NEG & $50(31)$ & $69(44)^{\star \star \star}$ & 90 & $97.1^{\star \star \star}$ & 100 & 100 \\
\hline CS & $29(18)$ & $14(9)^{\star \star \star}$ & 93.1 & 64.3 & 100 & 97.7 \\
\hline SS & $36(23)$ & 36 (23) & 86.1 & 77.8 & 100 & 100 \\
\hline MS & $27(17)$ & $15(10)^{\star \star \star}$ & 74.1 & $80^{\star \star}$ & 100 & 97.6 \\
\hline Group B (T2) & 72 & 55 & 68.1 & 69.1 & 97.2 & 98.2 \\
\hline NEG & $11(15)$ & $21(38)^{\star \star \star}$ & 90.9 & 85.7 & 100 & 100 \\
\hline CS & $15(21)$ & $6(11)^{\star \star \star}$ & 93.3 & 66.7 & 100 & 100 \\
\hline SS & $21(29)$ & $11(20)^{\star \star \star}$ & 61.9 & 63.6 & 100 & 100 \\
\hline MS & $9(13)$ & $8(15)$ & 33.3 & 50 & 77.8 & 87.5 \\
\hline
\end{tabular}

p-Values for comparisons between the pre-NBI group of patients and the NBI group in evaluation of margins are indicated as $\left(^{*}\right)$ when $<0.05$, $\left(^{* *}\right)$ when $<0.01$, and $\left(^{* * *}\right)$ when $<0.001$.

NEG, negative margin; CS, close superficial; $C D$, close deep; SS, positive single superficial; MS, positive multiple superficial; DEEP, positive deep margins.

borders, especially in the presence of CD margins, which significantly affected the recurrence rate in our series.

Single superficial margin infiltration had an overall impact on RFS, which was not significantly divergent from CS margins; it has to be noted, however, that, in the setting of T2, risk of recurrence tends to be significant, as 4 in 10 patients were found with recurrence during the course of their follow-up. Again, surveillance in these patients allowed thwarting any disease-related death and DSS was not statistically different from negative and close margins. Moreover, use of HDTV-NBI was especially beneficial in $\mathrm{T} 2$ patients, reducing the number of single superficial margin positivity.

It may be surmised that, even though CS and single superficial margins appear to be hallmarks of imperfect disease control, strict follow-up may be sufficient in avoiding the most severe outcomes. Further prospective studies could be aimed to test the validity of such approach against the routine application of adjuvant RT and/or repeated TLM. Our data, however, confirm that CD margins do have an impact on RFS and should not be overlooked.

Actually, even though fatal outcome is a rare occurrence in early glottic SCC (1), the vast majority of events clustered in patients with multiple superficial positive margins. This subpopulation, along with patients with deep margin infiltration, was also burdened by the highest recurrence rate. Moreover, in case of deep margin infiltration, we observed an increased need for total laryngectomy. Our results do not indicate a significant improvement in RFS with the application of adjuvant treatment. However, this analysis was limited by the reduced sample size and by patient selection bias; therefore, to draw any definite conclusions, large controlled prospective trials are needed.

This study has several limitations. First, it is retrospective in nature, analyzing pathologic data gathered at two different institutions over a relatively long time period. Moreover, follow-up was variable, as some patients, treated in the early 2000s, stopped attending visits after a long remission period, while others were recruited at relatively recent dates.

Overall, this study offers a possible key of interpretation of margin results in the framework of clinical decision during longterm follow-up. In particular, it suggests caution in presence of positivity of deep or multiple superficial margins, as these findings are hallmarks of possible disease persistence, especially in higher T categories. Patients with close margins, however, as well as those with single superficial margins, could undergo rigorous follow-up, as their recurrence rate is slightly higher.

\section{CONCLUSION}

Our data confirm that TLM for early-stage glottic SCC offers sound results in terms of DSS and RFS, as well as a high rate of OP. Survival-related parameters can be effectively stratified by margin status, even though larger studies are needed to definitively assess the opportunity, type, and outcomes of additional treatments in case of positive margins.

\section{CONSENT FOR PUBLICATION}

The manuscript does not contain any individual person's data.

\section{AVAILABILITY OF DATA AND MATERIALS}

The datasets analyzed during the current study are available from the corresponding author on reasonable request.

\section{ETHICS STATEMENT}

All patients signed a written informed consent, which was reviewed and approved by the local Ethics Committees and including the use of anonymized patient data for research purposes.

\section{AUTHOR CONTRIBUTIONS}

IF designed the study; FrancescoM, IF, FilippoM, MF, and AP collected and organized the clinical data. FF performed the statistical analysis. IF, FF, GiampieroP, CP, and GiorgioP drafted and reviewed the manuscript. All the authors read and approved the final manuscript.

\section{FUNDING}

This research received no specific grant from any funding agency in the public, commercial, or not-for-profit sectors. 


\section{REFERENCES}

1. Peretti G, Piazza C, Cocco D, De Benedetto L, Del Bon F, Redaelli De Zinis LO, et al. Transoral $\mathrm{CO}_{2}$ laser treatment for $\mathrm{T}(\mathrm{is})-\mathrm{T}(3)$ glottic cancer: the University of Brescia experience on 595 patients. Head Neck (2010) 32:977-83. doi:10.1002/hed.21278

2. Stoeckli SJ, Schnieper I, Huguenin P, Schmid S. Early glottic carcinoma: treatment according patient's preference? Head Neck (2003) 25:1051-6. doi:10.1002/hed.10323

3. Chera BS, Amdur RJ, Morris CG, Kirwan JM, Mendenhall WM. T1N0 to T2N0 squamous cell carcinoma of the glottic larynx treated with definitive radiotherapy. Int J Radiat Oncol Biol Phys (2010) 78:461-6. doi:10.1016/j. ijrobp.2009.08.066

4. Khan MK, Koyfman SA, Hunter GK, Reddy CA, Saxton JP. Definitive radiotherapy for early (T1-T2) glottic squamous cell carcinoma: a 20 year Cleveland clinic experience. Radiat Oncol (2012) 7:193. doi:10.1186/1748717X-7-193

5. Peretti G, Piazza C, Del Bon F, Mora R, Grazioli P, Barbieri D, et al. Function preservation using transoral laser surgery for T2-T3 glottic cancer: oncologic, vocal, and swallowing outcomes. Eur Arch Otorhinolaryngol (2013) 270:2275-81. doi:10.1007/s00405-013-2461-9

6. Canis M, Martin A, Ihler F, Wolff HA, Kron M, Matthias C, et al. Transoral laser microsurgery in treatment of pT2 and pT3 glottic laryngeal squamous cell carcinoma - results of 391 patients. Head Neck (2014) 36:859-66. doi:10.1002/hed.23389

7. Feng Y, Wang B, Wen S. Laser surgery versus radiotherapy for T1-T2N0 glottic cancer: a meta-analysis. ORL J Otorhinolaryngol Relat Spec (2011) 73:336-42. doi:10.1159/000327097

8. Misono S, Marmor S, Yueh B, Virnig BA. Treatment and survival in 10,429 patients with localized laryngeal cancer: a population-based analysis. Cancer (2014) 120:1810-7. doi:10.1002/cncr.28608

9. Warner L, Chudasama J, Kelly CG, Loughran S, McKenzie K, Wight R, et al. Radiotherapy versus open surgery versus endolaryngeal surgery (with or without laser) for early laryngeal squamous cell cancer. Cochrane Database Syst Rev (2014) 12:CD002027. doi:10.1002/14651858.CD002027.pub2

10. Schrijvers ML, van Riel EL, Langendijk JA, Dikkers FG, Schuuring E, van der Wal JE, et al. Higher laryngeal preservation rate after $\mathrm{CO}_{2}$ laser surgery compared with radiotherapy in Tla glottic laryngeal carcinoma. Head Neck (2009) 31:759-64. doi:10.1002/hed.21027

11. Zhang SY, Lu ZM, Luo XN, Chen LS, Ge PJ, Song XH, et al. Retrospective analysis of prognostic factors in 205 patients with laryngeal squamous cell carcinoma who underwent surgical treatment. PLoS One (2013) 8:e60157. doi:10.1371/journal.pone.0060157

12. Kitani Y, Kubota A, Furukawa M, Sato K. Prognostic factors for local control in patients receiving radiation therapy for early glottic cancer: anterior commissure involvement and effect of chemoradiotherapy. Eur Arch Otorhinolaryngol (2016) 273:1011-7. doi:10.1007/s00405-015-3579-8

13. Lim YJ, Wu HG, Kwon TK, Hah JH, Sung MW, Kim KH, et al. Long-term outcome of definitive radiotherapy for early glottic cancer: prognostic factors and patterns of local failure. Cancer Res Treat (2015) 47:862-70. doi:10.4143/ crt.2014.203

14. Ansarin M, Santoro L, Cattaneo A, Massaro MA, Calabrese L, Giugliano G, et al. Laser surgery for early glottic cancer: impact of margin status on local control and organ preservation. Arch Otolaryngol Head Neck Surg (2009) 135:385-90. doi:10.1001/archoto.2009.10

15. Lucioni M, Bertolin A, D’Ascanio L, Rizzotto G. Margin photocoagulation in laser surgery for early glottic cancer: impact on disease local control. Otolaryngol HeadNeckSurg (2012) 146:600-5. doi:10.1177/0194599811433270

16. Hoffmann C, Hans S, Sadoughi B, Brasnu D. Identifying outcome predictors of transoral laser cordectomy for early glottic cancer. Head Neck (2016) 38 (Suppl 1):E406-11. doi:10.1002/hed.24007

17. Edge S, Byrd DR, Compton CC, Fritz AG, Greene FL, Trotti A. AJCC Cancer Staging Manual. 7th ed. New York: Springer (2010).

18. Peretti G, Piazza C, Mensi MC, Magnoni L, Bolzoni A. Endoscopic treatment of cT2 glottic carcinoma: prognostic impact of different pT subcategories. Ann Otol Rhinol Laryngol (2005) 114:579-86. doi:10.1177/000348940511400801

19. Piazza C, Mangili S, Bon FD, Paderno A, Grazioli P, Barbieri D, et al. Preoperative clinical predictors of difficult laryngeal exposure for microlaryngoscopy: the Laryngoscore. Laryngoscope (2014) 124:2561-7. doi:10.1002/ lary. 24803

20. Garofolo S, Piazza C, Del Bon F, Mangili S, Guastini L, Mora F, et al. Intraoperative narrow band imaging better delineates superficial resection margins during transoral laser microsurgery for early glottic cancer. Ann Otol Rhinol Laryngol (2015) 124:294-8. doi:10.1177/0003489414556082

21. Remacle M, Eckel HE, Antonelli A, Brasnu D, Chevalier D, Friedrich G, et al. Endoscopic cordectomy. A proposal for a classification by the Working Committee, European Laryngological Society. Eur Arch Otorhinolaryngol (2000) 257:227-31. doi:10.1007/s004050050228

22. Remacle M, Van Haverbeke C, Eckel H, Bradley P, Chevalier D, Djukic V, et al. Proposal for revision of the European Laryngological Society classification of endoscopic cordectomies. Eur Arch Otorhinolaryngol (2007) 264:499-504. doi:10.1007/s00405-007-0304-2

23. Peretti G, Piazza C, Bolzoni A, Mensi MC, Rossini M, Parrinello G, et al. Analysis of recurrences in 322 Tis, T1, or T2 glottic carcinomas treated by carbon dioxide laser. Ann Otol Rhinol Laryngol (2004) 113:853-8. doi:10.1177/000348940411301101

Conflict of Interest Statement: The authors declare that the research was conducted in the absence of any commercial or financial relationships that could be construed as a potential conflict of interest.

Copyright (C) 2017 Fiz, Mazzola, Fiz, Marchi, Filauro, Paderno, Parrinello, Piazza and Peretti. This is an open-access article distributed under the terms of the Creative Commons Attribution License (CC BY). The use, distribution or reproduction in other forums is permitted, provided the original author(s) or licensor are credited and that the original publication in this journal is cited, in accordance with accepted academic practice. No use, distribution or reproduction is permitted which does not comply with these terms. 


\section{OPEN ACCESS}

Edited by:

Dietmar Thurnher,

Medizinische Universität Graz, Austria

Reviewed by:

Sandro J. Stoeckli,

Kantonsspital St. Gallen, Switzerland

Torahiko Nakashima,

Kyushu University, Japan

${ }^{*}$ Correspondence:

Vincent Vander Poorten

vincent.vanderpoorten@uzleuven.be

Specialty section:

This article was submitted to Head and Neck Cancer,

a section of the journal

Frontiers in Oncology

Received: 25 March 2018 Accepted: 11 May 2018

Published: 28 May 2018

Citation:

Meulemans J, Bijnens J, Delaere P and Vander Poorten V (2018)

Up-Front and Salvage Transoral Laser Microsurgery for Early Glottic Squamous Cell Carcinoma: A Single Centre Retrospective Case Series.

Front. Oncol. 8:186.

doi: 10.3389/fonc.2018.00186

\section{Up-Front and Salvage Transoral Laser Microsurgery for Early Glottic Squamous Cell Carcinoma: A Single Centre Retrospective Case Series}

\author{
Jeroen Meulemans ${ }^{1,2}$, Jacqueline Bijnens ${ }^{1,2}$, Pierre Delaere ${ }^{1}$ and Vincent Vander Poorten ${ }^{1,2 *}$ \\ 'Otorhinolaryngology-Head and Neck Surgery, University Hospital Leuven, Leuven, Belgium, ${ }^{2}$ Department of Oncology, \\ Section Head and Neck Oncology, KU Leuven, Leuven, Belgium
}

Introduction/aim: Transoral laser microsurgery (TLM) is a minimally invasive surgical alternative for radiotherapy (RT) in the primary management of early glottic cancer. More recently, TLM emerged also as a possible salvage treatment for selected radiorecurrent cancers. We reviewed outcomes of primary and salvage TLM performed in a Belgian tertiary referral center.

Patients and methods: A retrospective review of records from 142 consecutive patients who underwent TLM was performed. Oncologic outcomes were evaluated by means of descriptive statistics and Kaplan-Meier estimates. Variation of estimated outcomes between different subgroups was evaluated using Log-Rank analysis.

Results: Of 142 patients, 109 (76.8\%) underwent TLM as a primary treatment and 33 $(23.2 \%)$ were treated in a salvage setting for recurrent or second primary glottic cancer. CT classification in the up-front TLM group was cT1a in 72 (66.1\%), cT1b in 11 (10.1\%), and cT2 in 26 (23.9\%) patients. In the salvage group, patients were cT/rT classified as cT1arT1a in 17 (51.5\%), cT1b-rT1b in 1 (3.0\%), cT2-rT2 in 14 (42.4\%), and cT3-rT3 in 1 (3.0\%) patients. All patients were cNO. Second-look TLM was performed in 28 patients (19.7\%), and RT was associated as adjuvant therapy in 5 patients (3.5\%). Mean follow-up was 51.6 months ( $\mathrm{SD}=38.4$ months). Three-year overall survival $(\mathrm{OS})$ was $94.1 \%$ (SE $=2.2 \%$ ), 3-year disease-specific survival (DSS) 100\%, 3-year disease-free survival (DFS) 80.1\% (SE $=3.8 \%$ ), 3-year local recurrence-free survival (RFS) 81.0\% (SE = 3.7\%), and 3-year ultimate local control rate with laser alone 89.2\% (SE = 3.0\%). Upon subgroup analysis, no differences in OS, DSS, and DFS were observed between the up-front and salvage group (log rank; $p=0.306, p=0.298$, and $p=0.061$ respectively). However, local RFS and ultimate local control rate with laser alone were significantly higher in the primary treated TLM group (log rank, $p=0.014$ and $p=0.012$ ). Five-year laryngeal preservation rate was $89.7 \%$ $(\mathrm{SE}=3.5 \%)$ in the total population, $100 \%$ in the upfront group, and $64.9 \%$ (SE $=9.8 \%$ ) in the salvage group, a difference which proved statistically significant (Log-Rank, $p<0.001)$.

Conclusion: This retrospective study confirms excellent oncologic outcomes of up-front TLM for early glottic cancer. In the salvage setting, TLM allows avoidance of total laryngectomy in the majority of cases.

Keywords: conservation surgery, laryngeal cancer, salvage surgery, transoral laser microsurgery, squamous cell carcinoma 


\section{INTRODUCTION}

In the US and North-western European countries, early (T1-T2) glottic squamous cell carcinoma (SCC) has traditionally been treated most commonly with primary external beam radiotherapy (RT), yielding excellent functional and oncologic results. The reported rates of local control with RT alone for T1 glottic SCC range from 84 to $95 \%$ (1). For T2 glottic tumors, local control rates between 50 and $85 \%$ have been reported (1). However, transoral laser microsurgery (TLM) emerged as a surgical alternative to RT for the primary management of early glottic SCC. TLM was introduced as a minimally invasive therapeutic technique mounting a $\mathrm{CO}_{2}$ laser on an operating microscope for treatment of laryngeal lesions by Strong and Jako (2). In Europe, TLM for the treatment of glottic malignancies was initially mainly propagated by Steiner in the 1980s (3), who expanded the indications of TLM to all tumor categories of the upper aerodigestive tract (4). The main feature of TLM is the concept of tumor adapted resection: the tumor is transected and removed piecemeal through the laryngoscope. Transection of the tumor reveals the depth of tumor invasion and allows for clear visualization of tumor margins, resecting the tumor with an adequate margin while leaving as much healthy tissue as possible, the anatomy of the organ being less disturbed as compared with open transcervical/translaryngeal approaches. This minimizes the adverse functional impact, while leaving all salvage options open, including (chemo)radiotherapy or radical surgery. Nowadays, TLM has a proven track record in the primary management of glottic cancer, combining local control rates comparable to primary irradiation with excellent laryngeal preservation rates (1,5-9). More recently, TLM emerged as a possible salvage treatment for selected radiorecurrent laryngeal cancers (10-12). In this study, we review the outcomes of primary and salvage TLM performed in a Belgian tertiary referral center.

\section{PATIENTS AND METHODS}

\section{Patients}

A retrospective study was conducted at an academic tertiary referral hospital (Department of Otorhinolaryngology, Head and Neck Surgery, University Hospitals Leuven, Leuven, Belgium). This study was approved by and carried out in accordance with the recommendations of the Institutional Review Board (University Hospital Leuven Committee for Medical Ethics). Informed consent for retrospective studies with anonymized data is not required according to Belgian law. The records of all patients who were scheduled for primary or salvage TLM for suspected glottic malignancy between 1999 and 2016 were retrospectively reviewed and analyzed. Exclusion criteria were as follows: definitive pathology other than SCC (benign lesions, plasmacytoma, verrucous carcinoma, etc.) and TLM performed by a surgeon other than the senior surgeon (Vincent Vander Poorten). The selection of patients with primary early glottic SCC for TLM was based on a combination of (1) an estimate of tumor extent based on laryngoscopic and CT-graphic evaluation so that tumor resection would be possible with relatively low impact on the patient's voice and (2) patient's preference following this advice. The ultimate decision to submit a patient with glottic SCC to TLM rather than RT always resulted from a multidisciplinary tumor board discussion during which the patient's preferences were respected. As most patients were referred to our tertiary center following CT scanning and direct laryngoscopy and biopsy in a regional hospital, tumor board discussion was based on this referral information combined with thorough in-office laryngoscopy. If, based on this information, TLM seemed an adequate treatment option, a general tumor board agreement allows the surgeon to schedule a direct laryngoscopy and immediately proceed to TLM if an adequate resection is feasible. As such, procedures were partly planned as diagnostic laryngoscopy "with reserve." This means that already preoperatively some doubt about the feasibility of TLM existed, and the patient was clearly informed that the peroperative decision to proceed to therapeutic TLM would depend on adequate exposure, adequate resection deemed possible and an expected acceptable voice function. When these preconditions were peroperatively not fulfilled, the procedure remained a pure diagnostic laryngoscopy with biopsy retrieval and/or debulking and RT was offered as a therapeutic alternative. Before surgery, patients were staged and screened for synchronous upper aerodigestive tract tumors and distant disease using esophagogastroduodenoscopy, chest X-ray, and ultrasound of the liver or CT chest abdomen, according to our general work-up protocol for patients with head and neck cancer. T-status was determined according to the International Union for Cancer Control (UICC) 7th edition staging system for malignant head and neck tumors (13).

\section{Treatment}

All TLM procedures were performed by the same senior surgeon (Vincent Vander Poorten). Patients were under general anesthesia and ventilated using a small diameter endotracheal tube $(5.5$ or $6 \mathrm{~mm})$ or jet ventilation. Different closed laryngoscopes (Karl Storz, Tuttlingen, Germany) were used to achieve optimal exposure of the glottic area. Before the actual TLM procedure, the extent and location of the tumor was assessed using $30^{\circ}$ and $70^{\circ}$ endoscopes. TLM was performed using a $\mathrm{CO}_{2}$ laser (Model $40 \mathrm{C}$, Sharplan, Israël, later on AcuPulse Duo, Lumenis, Israël) equipped with a micromanipulator attached to the operating microscope (OPMI Vario, Zeiss, Göttingen, Germany). Resections were performed according to techniques described by Steiner $(3,4)$. Except from the smallest glottic lesions which were removed en bloc, a piecemeal resection was achieved with cutting through the tumor, allowing peroperative exploration of depth of invasion, taking a precise extra margin, while preserving as much healthy tissue as possible. TLM procedures were classified as recommended by the European Laryngological Society $(14,15)$. Patients treated by TLM in a salvage setting and resulting exposed laryngeal cartilage were administered antibiotics (moxifloxacin $400 \mathrm{mg}$ daily) during at least 10 days to prevent development of chondroradionecrosis (16). Following surgical treatment and pathologic examination of the resection specimen, the patients were re-discussed during the multidisciplinary tumor board meeting before any adjuvant therapy (second-look procedure or RT) was proposed. Secondlook procedures were reserved for patients with positive deep section margins and/or multiple positive superficial margins. 
Negative margins were defined as those with $>1 \mathrm{~mm}$ between margin and tumor, close margins were $\leq 1 \mathrm{~mm}$ from the tumor, and positive margins were margins with overt tumoral infiltration. Adjuvant RT was indicated in cases of massive tumoral infiltration of section margins combined with the surgeon's persuasion that no clear margins would be achieved with a second-look TLM procedure or in case of a second-look operation with definitive pathology showing again positive deep or superficial margins (no such case was encountered howeversee below). After termination of treatment, regular follow-up visits with rigid or flexible laryngoscopy were organized every 2 months during the first 2 years, every 3 months during the third year, every 4 months during the fourth year, and every 6 months thereafter. Baseline imaging of the neck (CT or MRI) was performed 3-4 months after treatment and was repeated 1 and 2 years after treatment to exclude locoregional recurrence in more advanced cases (pT2-pT3) (17). Yearly imaging of the chest (plain chest radiograph or CT chest) was performed to detect second primary pulmonary cancer.

\section{Data and Statistical Analysis}

The data related to patient, tumor and treatment characteristics, and oncologic and functional outcomes were retrieved anonymously from the patient's files. Information was collected on gender, ethyl and smoking history, previous treatment for head/ neck malignancies, previous treatment for glottic malignancies, cTNM classification, tumor extension [subglottic extension, supraglottic extension, involvement of anterior commissure (AC), and involvement of posterior commissure], type of cordectomy, laryngeal resection margins, tumor histology, pT classification, tubefeeding following TLM, tracheotomy following TLM, hospitalization duration, complications, adjuvant treatment, length of follow-up, tumor recurrence, glottic second primary occurrence, laryngeal preservation rate, and cause of death. Salvage setting TLM is defined as TLM procedures carried out for glottic SCC after previous RT in the head and neck region (glottic and nonglottic with inclusion of the glottis area in the irradiation field). According to the criteria of Warren and Gates and its modification by the National Cancer Institute, second primary SCC of the glottis was defined as a metachronous glottic SCC developing later than 60 months after the index diagnosis (18-20). As such, local recurrence after TLM was defined as malignancy in the same laryngeal subsite as the primary tumor diagnosed within 60 months after the diagnosis of the first glottic malignancy. Tumors developed later were considered glottic second primaries resulting from field cancerization. Data were statistically analyzed using SPSS version 22.0 statistical software (IBM Corp., Armonk, NY, USA). Laryngeal preservation rate at end of follow-up and local control rate at end of follow-up were compared between primary and salvage TLM patients using the chi-square test. Kaplan-Meier methods were used to estimate ultimate local control with laser alone, laryngeal preservation rate, overall survival (OS), disease-free survival (DFS), disease-specific survival (DSS), and local recurrence-free survival (RFS). The endpoint for DFS was the date of the first recurrence (local, regional, or distant). The endpoint for local RFS was the date of diagnosis of a first local recurrence. Univariate analysis using Log-Rank testing was employed to compare these data between different subgroups. Statistical significance was defined at the $p<0.05$ level.

\section{RESULTS}

\section{Patient and Treatment Characteristics}

142 patients were included, 133 (93.7\%) male and $9(6.3 \%)$ female patients. Mean patient age at time of TLM was 68 years (range 35-89 years, SD 11.3 years). TLM was performed as a primary or up-front treatment in 109 (76.2\%) cases whereas 33 (23.2\%) patients were treated in a salvage setting for recurrent glottic cancer after prior irradiation ( $n=28,84.8 \%$ of salvage cases) or for second primary cancer of the glottis after prior (chemo)radiation for a non-glottic head and neck cancer $(n=5,15.2 \%$ of salvage cases). No neck dissections were performed in accordance with current practice in treatment of early glottic SCC. Types of cordectomies [according to ELS classification $(14,15)]$ performed were as follows: type I ( $n=46,32.4 \%)$, type II $(n=21,14.8 \%)$, type III ( $n=41,28.9 \%)$, type IV $(n=7,4.9 \%)$, type Va $(n=14$, $9.9 \%)$, type $\mathrm{Vb}(n=1,0.7 \%)$, type $\mathrm{Vc}(n=3,2.1 \%)$, type $\mathrm{Vd}(n=1$, $0.7 \%)$, and type VI $(n=5,3.5 \%)$. Data about type of cordectomy performed were missing in three cases (2.1\%). Mean hospital stay (including day of the operation) was 1.5 days (range $1-8$ days, SD 0.8 days). No patients received a tracheotomy or a feeding tube. Only four patients (2.8\%) experienced mild complaints of aspiration, which was managed by temporarily thickening of fluids. One primarily treated patient developed chondronecrosis which was managed conservatively. After TLM, 109 patients (76.2\%) were submitted to a wait and see policy, with radiological follow-up in cases who proved peroperatively to be T2 or T3 or in case of any clinical doubt. Twenty-eight patients (19.7\%) were scheduled for a second-look TLM procedure with re-resection because of a compromised deep margin or multiple superficial margins positive for SCC with an invasive component. Of interest, not a single second-look TLM procedure yielded residual malignancy after pathological examination. In five cases (3.5\%), second-look TLM was renounced by the treating surgeon due to the expected low probability of radicality in a voice preserving setting, and RT was preferred after multidisciplinary discussion.

\section{Tumor Characteristics}

Based on preoperative clinical and radiological findings, cT classification in the up-front TLM group was cT1a in $72(66.1 \%)$, cT1b in $11(10.1 \%)$, and cT2 in $26(22.9 \%)$ patients. In the salvage group, patients were cT/rT classified as cT1a-rT1a in $17(51.5 \%)$, cT1b-rT1b in 1 (3.0\%), cT2-rT2 in $14(42.4 \%)$, and cT3-rT3 in $1(3.0 \%)$ patients. All patients were cN0. Retrospective review revealed that, due to the absence of a standardized registration protocol, accurate description of tumor extent as observed during the TLM procedure was often lacking. Data on absence or presence of subglottic extension, supraglottic extension and involvement of the anterior and posterior commissure were lacking in 96 (67.6\%), 102 (71.8\%), $67(47.2 \%)$, and $65(45.8 \%)$ cases, respectively. Of the patients with detailed data on tumor extension available, involvement of the aforementioned laryngeal subregions was present in 
$41.3,32.5,57.3$, and $6.5 \%$, respectively. After resection, the pathologist judged the margins as clear in 30 patients $(21.1 \%)$, close in 9 patients $(6.3 \%)$, positive in 50 cases $(35.2 \%)$, and nonevaluable (due to laser coagulation artifacts and/or problems in orienting) in 53 patients (37.3\%). Patients were definitively classified as carcinoma in situ (CIS) ( $N=35,24.6 \%)$, pT1a/ rpT1a $(N=69 ; 48.6 \%)$, pT1b/rpT1b $(N=6 ; 4.2 \%)$, and pT2/ $\operatorname{rpT} 2(N=32 ; 22.5 \%)$.

\section{Oncologic Outcome and Survival}

Mean follow-up for the overall population was 51.6 months (range 0-187 months, SD 38.4 months). Death occurred during follow-up in 32 patients (22.5\% of the total population). In the up-front TLM group, 21 deaths occurred with 2 deaths (9.1\% of deaths in this subgroup) being related to the glottic cancer, 1 death due to distant disease and the other related to progression of a third recurrence with the patient opting for a palliative setting. In the salvage TLM group, 2 out of 11 reported deaths was disease related (18.2\% of deaths in this subgroup). In the upfront TLM group, 15 patients (13.8\%) developed a local recurrence, $2(1.8 \%)$ a regional recurrence, $1(0.9 \%)$ a locoregional recurrence, and 1 patient $(0.9 \%)$ developed distant disease. Four primarily treated patients $(3.7 \%)$ developed a glottic second primary malignancy (time between diagnosis of the primary glottic cancer and the second primary glottic cancer $>60$ months). Patients with local recurrence were salvaged with a redo TLM procedure $(n=8)$, external beam irradiation of the larynx $(n=4)$, or combined TLM and RT $(n=3)$. Patients with second primary glottic cancer were salvaged with TLM $(n=2)$, irradiation $(n=1)$, or combined treatment $(n=1)$. Eventually, one total laryngectomy was performed during the course of disease in the up-front group in a patient who suffered from serious aspiration after having been irradiated for second primary glottic cancer. In the salvage group, 12 patients (36.4\%) developed local recurrence of whom 3 were again salvaged with redo TLM and 8 with a total laryngectomy. Mean time interval between treatment and diagnosis of recurrence was 25 months (SD 16.5 months) in the primary group and 23.9 months (SD 17.9 months) in the salvage group (one-way ANOVA, $p=0.864$ ). The difference in probability of local recurrence during followup between both patient groups proved statistically significant (chi-square; $p=0.004$ ).

In a first analysis including second primary glottic cancers (see above: occurring $>60$ months following primary treatment) as failure, local control at the end of follow-up after one TLM treatment was $75.0 \%$ in the total population; this outcome was better (trend, not significant) in the up-front group (control rate of $78.6 \%$ ) than in the salvage group (control rate of 63.6\%) (chisquare, $p=0.083$ ). This local control rate at the end of follow-up rose to $83.8 \%$ after eventual additional laser procedures ("ultimate local control rate with laser alone"). This local control rate with laser alone proved significantly better in the up-front TLM group $(87.4 \%)$ when compared with the salvage group $(72.7 \%)$ (chi-square, $p=0.047$ ).

In a second analysis, looking at local control rates calculated by only including real local recurrences and excluding glottic second primaries, local control rate after one session of TLM was $80.3 \%$ in the total population ( $85.6 \%$ in primary group and $63.6 \%$ in salvage group, $p=0.006$ ), and ultimate local control with laser alone (including eventual additional laser procedures) was $87.6 \%$ in the total population $(92.3 \%$ in primary group and $72.7 \%$ in salvage group, $p=0.003)$.

Five-year local control rate with laser alone (Kaplan-Meier), excluding glottic second primaries, was $80.9 \%(\mathrm{SE}=4.5 \%)$ in the overall group and proved significantly higher in the primary TLM group $(87.8 \%, \mathrm{SE}=4.3 \%)$ compared with the salvage TLM group $(65.3 \%, \mathrm{SE}=9.8 \%)(\log \operatorname{rank}, p=0.012)$ (Figure 1). Kaplan-Meier estimated 5-year laryngeal preservation rate was $100 \%$ in the up-front group and $64.9 \%$ (SE = 9.8\%) in the salvage group, a difference that proved statistically significant (Log-Rank, $p<0.001$ ) (Figure 2). Looking in particular at patients who underwent a second-look procedure $(n=28,6$ salvage cases and 22 primary cases), 5 developed a local recurrence ( 3 primary cases and 2 salvage cases) of whom the 2 salvage cases were salvaged with a total laryngectomy. In the total patient group, 3-year OS was $94.1 \%(\mathrm{SE}=2.2 \%)$, 3-year DSS 100\%, 3-year DFS 80.1\% (SE = 3.8\%), and 3-year local RFS was $81.0 \%$ (SE $=3.9 \%)$. Three-year OS in the up-front treated patient group was $94.3 \%(\mathrm{SE}=2.5 \%)$ while the 3 -year OS in the salvage group was $93.4 \%(\mathrm{SE}=4.5 \%)(\log$-Rank test, $p=0.306)$ (Figure 3). Three-year DSS was $100 \%$ both in the up-front TLM and salvage patient group (Log-Rank test, $p=0.298$ ). Three-year DFS was $83.4 \%(\mathrm{SE}=4.1 \%)$ in the primary treated group and $70.4 \%(\mathrm{SE}=8.3 \%)$ in the salvage group (Log-Rank test, $p=0.061)$. Three-year local RFS was $84.6 \%(\mathrm{SE}=4.0 \%)$ after primary TLM and $70.4 \%(\mathrm{SE}=8.3)$ after salvage TLM (Log-Rank test, $p=0.014$ ). As such, local RFS was significantly higher in the primary group when compared with the salvage group (Figure 4). Three- and five-year survival rates, laryngeal preservation rates, and local control rates with laser alone are summarized in Table 1.

When the salvage group was restricted to patients with a local recurrence after previous RT for a glottic cancer $(n=28)$, thus excluding those patients with TLM for glottic carcinoma after having been irradiated for a non-glottic head and neck cancer $(n=5)$, the oncologic results in the salvage group did not change significantly when compared with the total salvage group $(n=33)$ upon Log-Rank analysis (DFS: $p=0.987$, OS: $p=0.983$, DSS: $p=0.929$, laryngeal preservation rate: $p=0.796$, local control rate with laser alone: $p=0.870$ ).

After further univariate analysis, section margin status, cT classification, involvement of the AC, and subglottic extension did not seem to influence local control with laser alone, DFS, or local RFS. Surprisingly, no significant higher local control with laser alone, DFS, or local RFS were observed in patients with CIS upon definitive pathological examination when compared with invasive SCC. Results of these subgroup analyses are illustrated in Table $\mathbf{2}$ and Figures 5 and $\mathbf{6 .}$

\section{DISCUSSION}

Since the 1980s, TLM emerged as a surgical alternative to RT for the primary management of early glottic SCC and has nowadays a proven track record in the primary management 


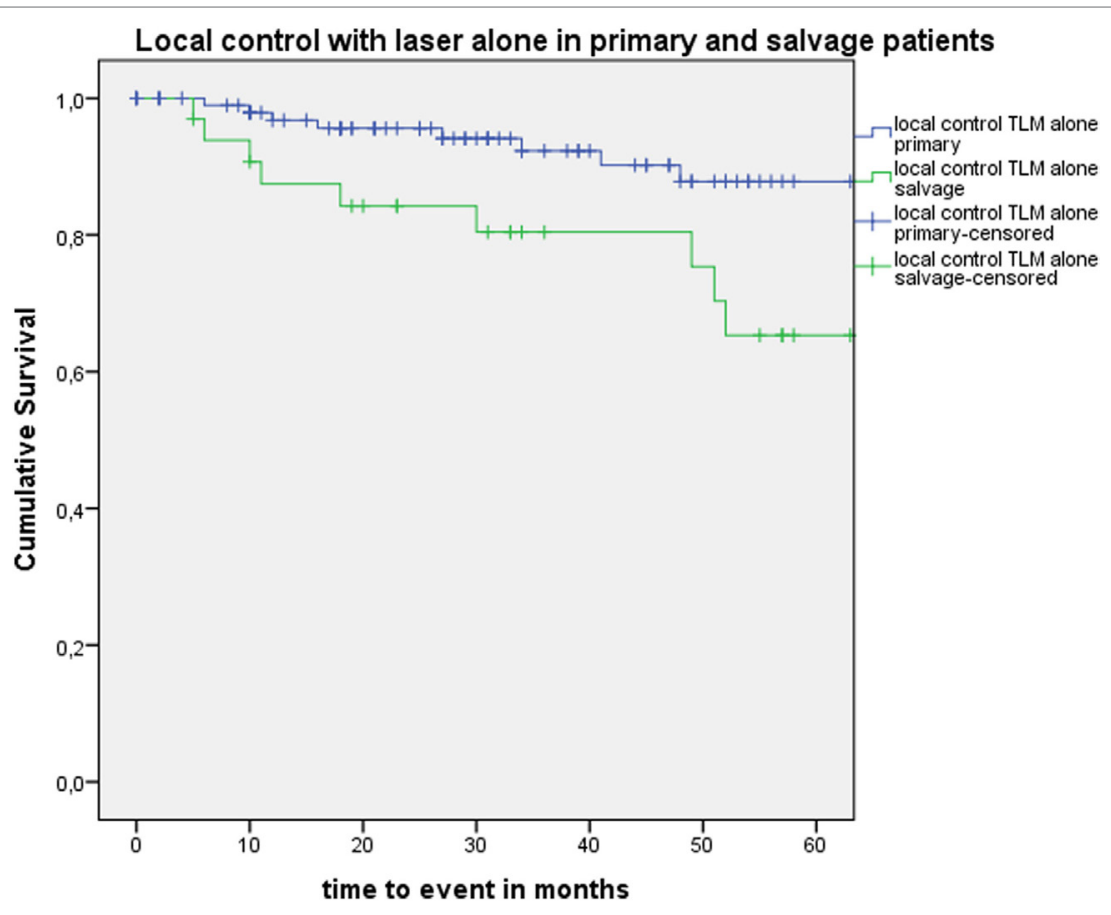

FIGURE 1 | Kaplan-Meier curve illustrating local control with laser alone in patients treated with up-front or primary transoral laser microsurgery (TLM) (blue) and salvage TLM (green). Local control with laser alone was significantly higher in the primary group when compared with the salvage group (Log-Rank test, $p=0.012$ ).

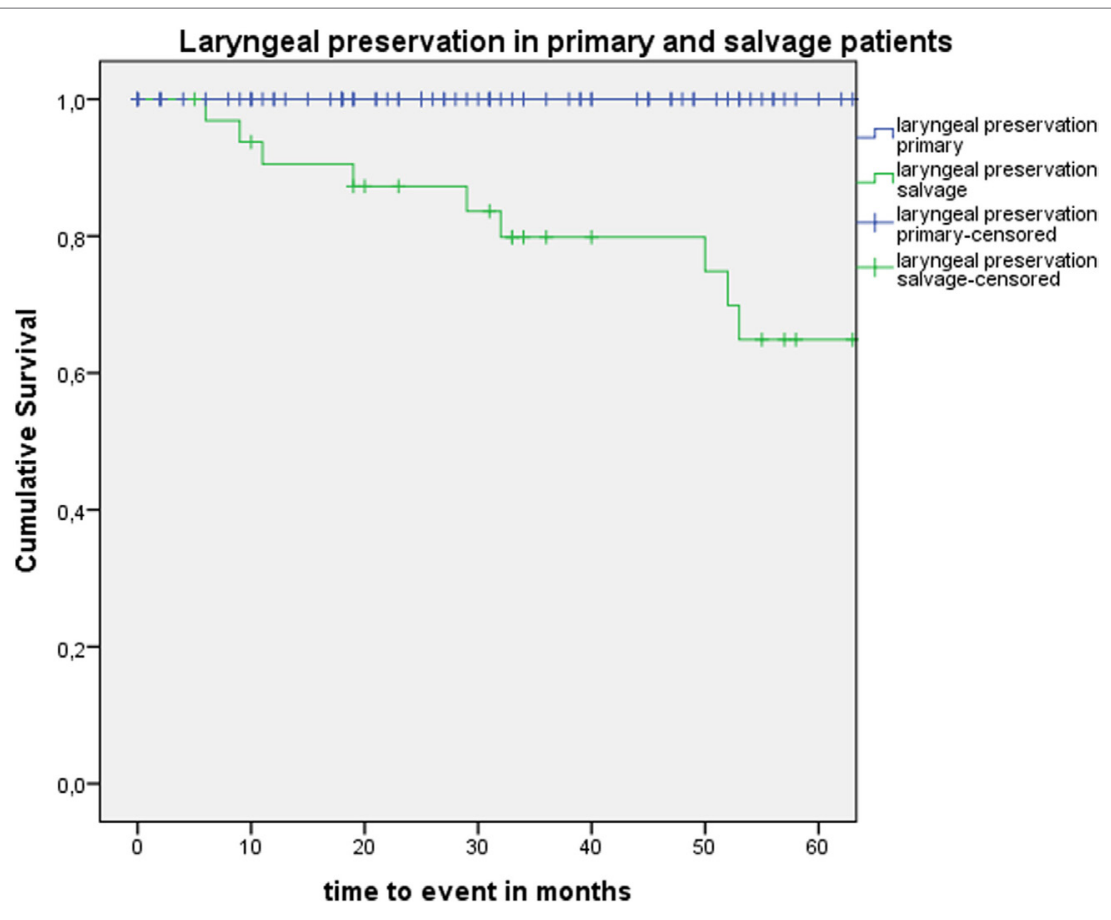

FIGURE 2 | Kaplan-Meier curve illustrating laryngeal preservation in patients treated with up-front or primary transoral laser microsurgery (TLM) (blue) and salvage TLM (green). Laryngeal preservation was significantly higher in the primary group when compared with the salvage group (Log-Rank test, $p<0.001)$.

of glottic cancer, combining high local control with excellent laryngeal preservation rates $(1,5-9,21)$. In this single-center retrospective case series, we report on the oncologic outcomes of our 142 first TLM procedures, hence this includes patients at the beginning of the learning curve and also including primary cases as well as salvage cases. As expected in a population of 


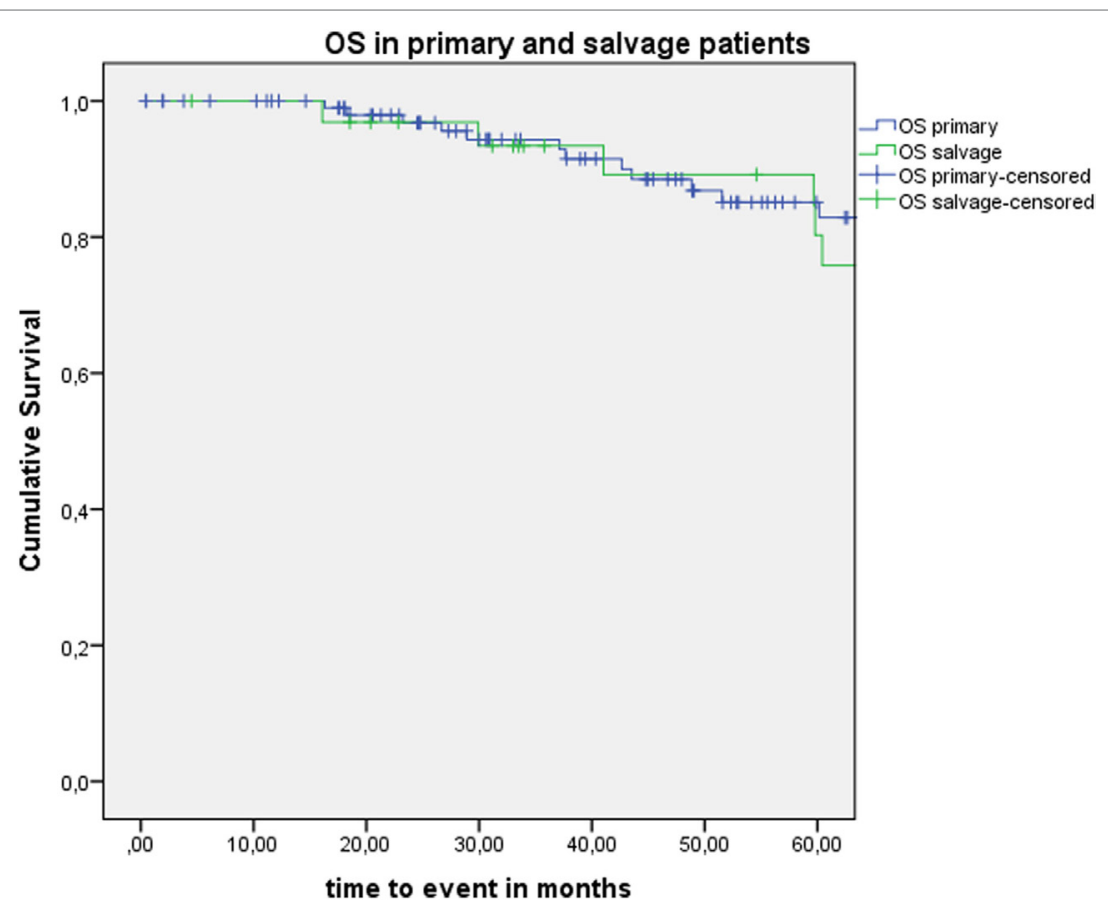

FIGURE 3 | Kaplan-Meier curve illustrating overall survival (OS) in patients treated with up-front or primary transoral laser microsurgery (TLM) (blue) and salvage TLM (green). No difference in OS is observed between the primary and salvage group (Log-Rank test, $p=0.306$ ).

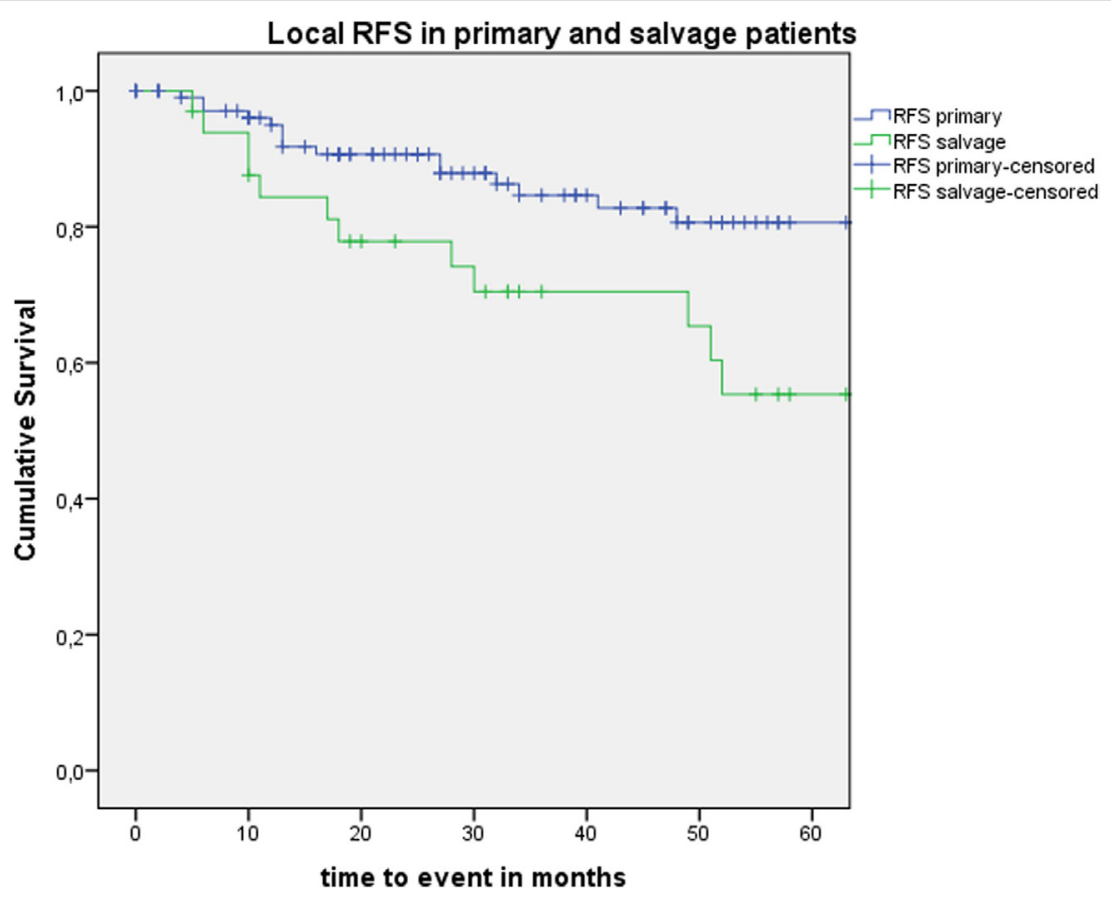

FIGURE 4 | Kaplan-Meier curve illustrating local recurrence-free survival (RFS) in patients treated with up-front or primary transoral laser microsurgery (TLM) (blue) and salvage TLM (green). Local RFS was significantly higher in the primary group when compared with the salvage group (Log-Rank test, $p=0.014$ ).

patients with early glottic carcinoma, 3- and 5-year DSS and OS proved to be excellent in our series, both in the up-front TLM group and in the salvage TLM group. Local recurrence was observed with high predominance over regional recurrence or distant disease, which is another logical finding in this patient group. Three- and five-year ultimate local control 
TABLE 1 | 3- and 5-year local control with laser alone, laryngeal preservation, and survival estimates (Kaplan-Meier) in the total population, the primary TLM group, and the salvage TLM group.

\begin{tabular}{|c|c|c|}
\hline & 3 years & 5 years \\
\hline $\begin{array}{l}\text { Local control laser alone total } \\
\text { population }\end{array}$ & $89.2 \%(\mathrm{SE}=3.0 \%)$ & $80.9 \%(\mathrm{SE}=4.5 \%)$ \\
\hline Local control laser alone primary & $92.3 \%(\mathrm{SE}=3.1 \%)$ & $87.8 \%(\mathrm{SE}=4.3 \%)$ \\
\hline Local control laser alone salvage & $80.4 \%(S E=7.2 \%)$ & $65.3 \%(\mathrm{SE}=9.8 \%)$ \\
\hline $\begin{array}{l}\text { Laryngeal preservation total } \\
\text { population }\end{array}$ & $94.6 \%(\mathrm{SE}=2.2 \%)$ & $89.7 \%(\mathrm{SE}=3.5 \%)$ \\
\hline Laryngeal preservation primary & $100 \%$ & $100 \%$ \\
\hline Laryngeal preservation salvage & $79.8 \%(\mathrm{SE}=7.4 \%)$ & $64.9 \%(\mathrm{SE}=9.8 \%)$ \\
\hline OS total population & $94.1 \%(\mathrm{SE}=2.2 \%)$ & $83.3 \%(\mathrm{SE}=3.9 \%)$ \\
\hline OS primary & $94.3 \%(\mathrm{SE}=2.5 \%)$ & $85.1 \%(\mathrm{SE}=4.2 \%)$ \\
\hline OS salvage & $93.4 \%(\mathrm{SE}=4.5 \%)$ & $80.3 \%(\mathrm{SE}=8.0 \%)$ \\
\hline DSS total population & $100.0 \%$ & $97.6 \%(\mathrm{SE}=1.7 \%)$ \\
\hline DSS primary & $100.0 \%$ & $98.4 \%(\mathrm{SE}=1.6 \%)$ \\
\hline DSS salvage & $100.0 \%$ & $95.5 \%(\mathrm{SE}=4.4 \%)$ \\
\hline DFS total population & $80.1 \%(\mathrm{SE}=3.8 \%)$ & $68.1 \%(\mathrm{SE}=5.1 \%)$ \\
\hline DFS primary & $83.4 \%(\mathrm{SE}=4.1 \%)$ & $72.9 \%(\mathrm{SE}=5.8 \%)$ \\
\hline DFS salvage & $70.4 \%(\mathrm{SE}=8.3 \%)$ & $55.3 \%(\mathrm{SE}=10.1 \%)$ \\
\hline RFS total population & $81.0 \%(\mathrm{SE}=3.7 \%)$ & $73.4 \%(\mathrm{SE}=4.7 \%)$ \\
\hline RFS primary & $84.6 \%(\mathrm{SE}=4.0 \%)$ & $80.6 \%(\mathrm{SE}=4.7 \%)$ \\
\hline RFS salvage & $70.4 \%(\mathrm{SE}=8.3 \%)$ & $55.3 \%(\mathrm{SE}=10.1 \%)$ \\
\hline
\end{tabular}

DFS, disease-free survival; DSS, disease-specific survival; OS, overall survival; RFS, recurrence-free survival; TLM, transoral laser microsurgery.

TABLE 2 | $p$-Values after comparison of local control with laser alone, DFS, and local RFS between different subgroups using Log-Rank test.

\begin{tabular}{lccc}
\hline $\begin{array}{l}\text { Univariate analysis } \\
\text { (Log-Rank) }\end{array}$ & $\begin{array}{c}\text { Local control with } \\
\text { laser alone }\end{array}$ & DFS & Local RFS \\
\hline CT classification & $p=0.696$ & $p=0.690$ & $p=0.726$ \\
pT classification & $p=0.302$ & $p=0.628$ & $p=0.583$ \\
Subglottic extension & $p=0.244$ & $p=0.540$ & $p=0.927$ \\
Involvement of AC & $p=0.588$ & $p=0.288$ & $p=0.414$ \\
CIS versus invasive SCC & $p=0.103$ & $p=0.530$ & $p=0.715$ \\
Section margin status & $p=0.663$ & $p=0.961$ & $p=0.927$ \\
\hline
\end{tabular}

$A C$, anterior commissure; CIS, carcinoma in situ; SCC, squamous cell carcinoma; RFS, recurrence-free survival; DFS, disease-free survival.

rates (excluding glottic second primary cancers) obtained by "laser only treatment" ( 1 or successive TLM procedures) were satisfying and proved significantly better in the up-front TLM group $(92.3 \%$ at 3 years and $87.8 \%$ at 5 years) when compared with the salvage group ( $80.4 \%$ at 3 years and $65.3 \%$ at 5 years) (Log-Rank; $p=0.012$ ). However, mainly due to the adjuvant use of RT in patients who could not be cured by TLM procedures alone, 3- and 5-year laryngeal preservation rates in the up-front TLM group raised to an excellent 100 versus $79.8 \%$ (3 years) and $64.9 \%$ (5 years) in the salvage group (Log-Rank, $p<0.001)$. This excellent laryngeal preservation rate in primarily treated patients is comparable to the rates described in the literature $(6,7,9,21,22)$ (ranging between 95 and 97.1\%) and clearly illustrates the long-term benefit with regards to laryngeal preservation of primary TLM treatment, leaving all salvage options open, including RT, should the tumor recur. In this aspect, TLM and RT are not mere alternatives to each other but are complimentary treatments in selected patients, leading to optimal oncologic outcomes. This also stresses the importance of a multidisciplinary approach of the patient with early glottic cancer. Looking at oncologic results of primary TLM reported in the literature, our 5-year ultimate local control rate with laser alone $(87.8 \%)$ is comparable to the ultimate local control rate with laser alone as reported by Peretti et al. (6) $(92.7 \%)$. Our 5 -year DFS is $72.9 \%$, which is rather low when compared with series by Peretti et al. (6) (81.3\%) and Ansarin et al. (7) (85.3\%). However, the overall incidence of local recurrences during follow-up in primary treated patients (13.8\%) is comparable to the incidence reported by Ansarin et al. (7) (12.2\%) but lower than reported by Schrijvers et al. (21) (27\%). Our estimated 5-year laryngeal preservation rate (100\%) and 5-year DSS (98.4\%) are among the best described in the literature. The dichotomy between a lower ultimate local control rate with laser alone on one hand and excellent DSS and laryngeal preservation rate on the other hand is due to a higher tendency in our centre to administer RT, either as an adjuvant treatment in patients with obvious section margin involvement or as a salvage option in cases of recurrence after primary TLM, when compared with other centers with high local control rates with laser alone, where redo laser procedures are preferred in these instances. The contrast between lower DFS but very high laryngeal preservation rate is a good illustration of the effectiveness of additional laser procedures and/or RT in saving the larynx in case of local recurrence.

Of particular interest in this series is the high amount of patients treated by TLM in a salvage setting $(N=33$ or $23.2 \%$ of the total population). In the literature, the evidence on TLM as a salvage treatment for radiorecurrent laryngeal cancer is scarce, the series being retrospective and including small numbers of highly selected patients $(16,23-32)$. When compared with these reports, our series report on a substantial number of patients with radiorecurrent glottic cancer. A recent literature review on salvage TLM including all relevant literature concluded to local control rates with TLM alone varying between 50 and $87 \%$, with a weighted average of $67 \%$. The rates of definite laryngeal preservation, as obtained after TLM alone or a combination of TLM and salvage open partial laryngectomy, ranged from 50 to $94 \%$ with a weighted average of $73 \%$ (10). In our salvage group, 5-year local control with laser alone was $65.3 \%$, and 5-year laryngeal preservation rate was $64.9 \%$. Three-year estimates of OS in the literature range from 67.5 to $93.7 \%$ and 5 -year estimates from 53 to $91 \%$; for DSS, 3- and 5-year estimates both ranging between 68.6 and $100 \%$ are reported (10). In a systematic review, Ramakrishnan et al. calculated pooled mean estimates at 24 months for OS of $74.8 \%$ and for DFS of $70.9 \%$ (12). In our salvage patient series, 3- and 5-year OS were 93.4 and $80.3 \%$, respectively; 3 - and 5-year DSS were 100 and 95.5\%, respectively; and 3 - and 5-year DFS were 70.4 and 55.3\%, respectively. These oncologic results are among the most favorable described in the literature and clearly illustrate the potential of salvage TLM to control the disease and avoid a total laryngectomy in well selected patients. Concerning resection margins after TLM for early glottic carcinoma, the relationship between section margin status and recurrence rate is unclear since a significant proportion of patients with reportedly involved margins never 


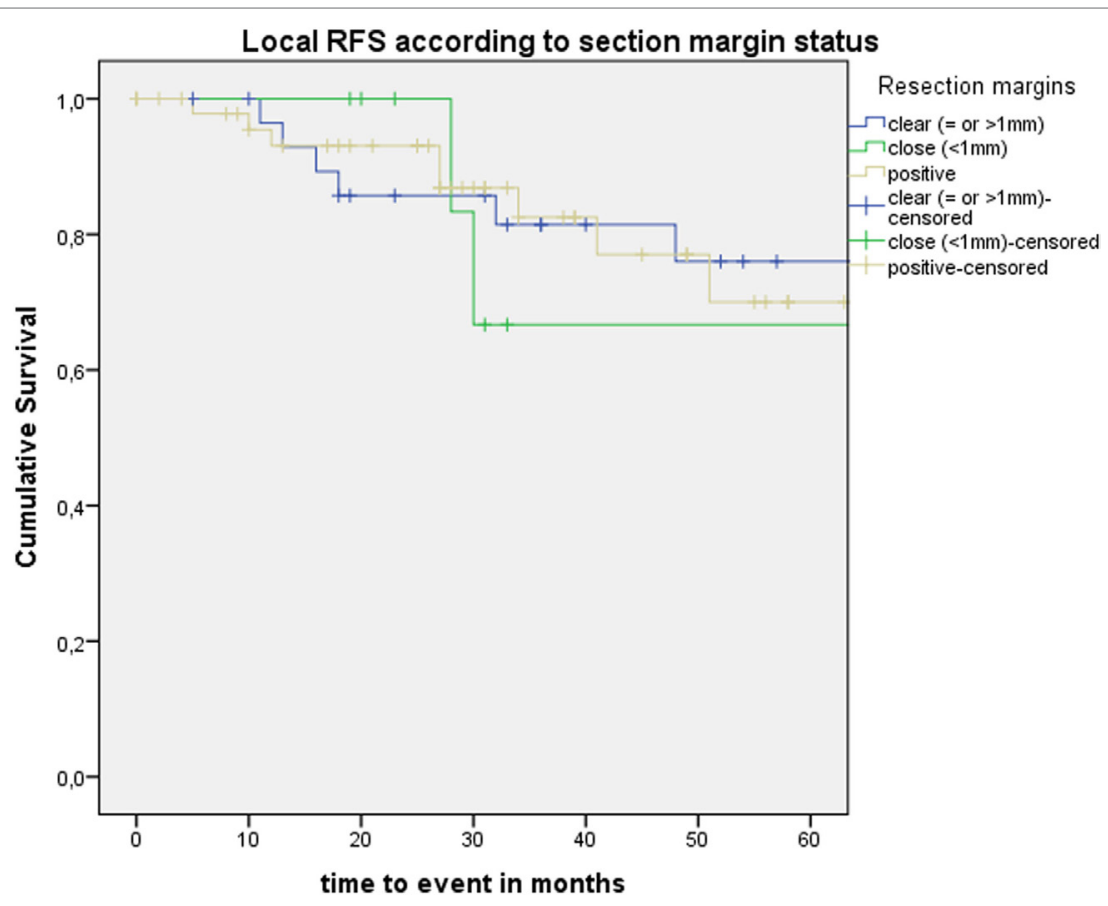

FIGURE 5 | Kaplan-Meier curve illustrating local recurrence-free survival (RFS) according to section margin status.

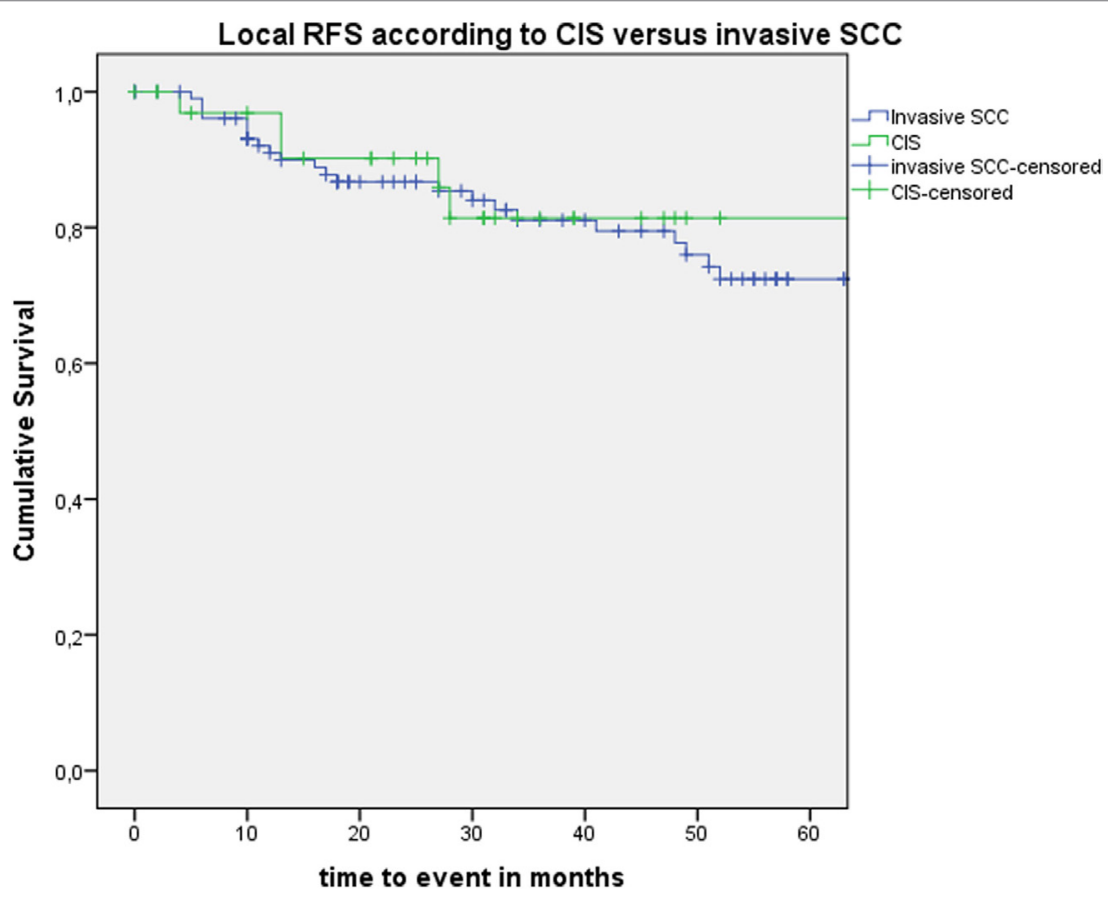

FIGURE 6 | Kaplan-Meier curve illustrating local recurrence-free survival (RFS) according to presence of carcinoma in situ (CIS) or invasive squamous cell carcinoma (SCC).

develop disease recurrence. In some patient series, close and positive margins were found to be independent risk factors for recurrence and poorer survival rates, while other studies were not able to confirm this negative impact of involved margin status (33). In an interesting recent study, Fiz et al. observed a reduction of DSS and RFS in pTis-T1b patients treated by TLM and positive multiple superficial and positive deep margins. In pT2 patients, DSS was reduced in positive multiple superficial 
margins, and RFS was reduced in positive single superficial, positive multiple superficial, and positive deep margins (34). In a review on TLM of early glottic cancer by Sjögren, rates of positive or inadequate margins between 24 and 51\% were identified (22). In our series, margins were judged clear in 30 patients $(21.1 \%)$, close in 9 patients $(6.3 \%)$, positive in 50 cases $(35.2 \%)$, and non-evaluable in 53 patients (37.3\%). Secondlook procedures were considered when multiple superficial margins and/or the deep margin were compromised, but apart from this pathologic margin status, the intraoperative opinion of the experienced surgeon on resection radicality seems the most important factor in decision-making. Eventually, a negative selection of $28(19.7 \%)$ patients (where the surgeon had the most doubt on the radicality of the resection) was scheduled for a second-look TLM procedure with re-resection but not a single second-look TLM procedure yielded residual malignancy, suggesting a high rate of initially "false positive" involved section margins. This finding is supported by data from Ansarin et al. who observed persistence of disease in the resection specimens obtained by second-look TLM because of positive or close margins or dysplasia at margins in only 6 out of 90 patients $(6.6 \%)$. Interestingly, they observed upon multivariate analysis that initial close to positive margins negatively influenced local recurrences, but also suggested that in this group with compromised margins, no difference in local recurrence rate was found between patients who underwent adjuvant RT or second-look procedure and those who underwent follow-up (7). Although these data need to be interpreted with caution due to small subpopulations and plausible selection bias with more aggressive tumors more likely to end up in the second-look or RT-arm, they put in doubt the added benefit of performing second-look procedures. However, until more robust data are available, is seems a sensible practice to perform second-look procedures for at least compromised deep margins. This pragmatic approach is supported by data from Peretti et al. who observed a low impact of superficial positive margins on local control with laser and organ preservation rates compared with deep infiltration or residual disease (6). The high proportion of margins considered by the pathologist as too difficult to assess and consequently too doubtful to make a reliable statement about is due to the small specimen sizes, laser coagulation and carbonization artifacts, tissue retraction, and orientation issues, which are well known particular problems after TLM. After having been confronted with this high rate of non-evaluable margins, our group searched for ways to counter these problems related to specimen orientation and evaluation. Inspired by earlier research in which a way to fix and orient surgical specimens on dehydrated cucumber was described $(35,36)$, we started to fix and meticulously orient the surgical samples on pig liver slices immediately after resection. The carrier-mounted specimens are sent to the pathologist, accompanied by a photograph of the tumor in situ. This new technique is assessed in an ongoing prospective trial and awaits objective validation. In our series, AC involvement could not be identified as a negative prognosticator. Although AC involvement has been found to be a risk factor for local recurrence (37), some large series could not confirm this negative impact on local control $(6,7)$. It seems appropriate to differentiate between tumors with AC involvement in the horizontal plane and those tumors in which the AC involvement extends in the vertical plane to the subglottic and/or supraglottic region. The latter tumor group carries a risk of poorer local control due to the close relationship with the underlying visceral spaces (pre-epiglottic space and subglottic area) (38). In radiorecurrent carcinoma, this anterior transcommissural extension was identified as a negative predictor on OS (39).

Evidently, our study has limitations. As a retrospective study, inherent selection bias cannot be excluded. Moreover, because of the small study population and small subgroups, multivariate analysis was not possible. As the primary TLM group and salvage TLM group are two completely different cohorts, statistical comparison needs to be interpreted with caution. However, a rudimentary statistical comparison of outcomes is of interest for the reader because it confirms some obvious differences between primary and salvage groups (e.g., lower probability of local recurrence during follow-up, better local control with laser alone, better laryngeal preservation rate, and higher local RFS in the primary group) but it also illustrates that other oncologic outcomes of the difficult-to-treat salvage group are not worse when compared with the primary group (OS, DSS, and RFS). This is an important finding, as indications of TLM are progressively stretched and salvage TLM is becoming more widespread. As such, the comparison of both groups only tries to give the reader an idea about how results of TLM in a salvage group relate to those in a primary group, which is useful information both for the surgeon as for the patient. During data retrieval, we observed high rates of lacking data concerning the detailed extension of the tumor as observed during the TLM procedure (supraglottic and subglottic extension, involvement of the anterior and posterior commissure), making subgroup analysis even more difficult. As a reaction to this finding, we are evolving to a systematic and standardized way of reporting visualized tumor extension during TLM, making more detailed future evaluations possible. Another drawback of our study is the lack of objective pre- and postoperative voice assessments. As complications after TLM procedures, both in the primary and salvage setting, are very rare, postoperative voice quality warrants most attention when addressing functional outcomes of TLM. In our study, no valuable statements could be made on this important functional aspect due to the lack of systematically performed pre- and postoperative vocal assessment. Finally, as mentioned earlier, a high rate of non-evaluable margins were encountered due to orientation and processing difficulties experienced by the pathologist.

\section{CONCLUSION}

This single-center retrospective case series confirms excellent definitive oncologic outcomes of up-front TLM for early glottic cancer with a 5-year laryngeal preservation rate of $100 \%$ and 5 -year DSS of $98.4 \%$. In the salvage setting, TLM allows avoidance of total laryngectomy in $64.9 \%$ of cases after 5 years. Upon univariate analysis, local control rate with laser alone, laryngeal preservation rate, and local RFS were significantly higher in the 
up-front TLM group when compared with the salvage TLM group, while OS, DSS, and DFS did not show significant differences between the subgroups.

\section{ETHICS STATEMENT}

This study was carried out in accordance with the recommendations of the Institutional Review Board (University Hospital Leuven). The protocol was approved by the Committee for Medical Ethics of the University Hospitals Leuven. Informed

\section{REFERENCES}

1. Hartl DM, Ferlito A, Brasnu DF, Langendijk JA, Rinaldo A, Silver CE, et al. Evidence-based review of treatment options for patients with glottic cancer. Head Neck (2011) 33(11):1638-48. doi:10.1002/hed.21528

2. Strong MS, Jako GJ. Laser surgery in the larynx. Early clinical experience with continuous $\mathrm{CO}_{2}$ laser. Ann Otol Rhinol Laryngol (1972) 81(6):791-8. doi:10.1177/000348947208100606

3. Steiner W. Results of curative laser microsurgery of laryngeal carcinomas. Am J Otolaryngol (1993) 14(2):116-21. doi:10.1016/0196-0709(93)90050-H

4. Steiner W. Experience in endoscopic laser surgery of malignant tumours of the upper aero-digestive tract. Adv Otorhinolaryngol (1988) 39:135-44.

5. Ambrosch $P$. The role of laser microsurgery in the treatment of laryngeal cancer. Curr Opin Otolaryngol Head Neck Surg (2007) 15(2):82-8. doi:10.1097/ MOO.0b013e3280147336

6. Peretti G, Piazza C, Cocco D, De Benedetto L, Del Bon F, Redaelli De Zinis LO, et al. Transoral $\mathrm{CO}(2)$ laser treatment for T(is)-T(3) glottic cancer: the University of Brescia experience on 595 patients. Head Neck (2010) 32(8):977-83. doi:10.1002/hed.21278

7. Ansarin M, Cattaneo A, De Benedetto L, Zorzi S, Lombardi F, Alterio D, et al. Retrospective analysis of factors influencing oncologic outcome in 590 patients with early-intermediate glottic cancer treated by transoral laser microsurgery. Head Neck (2017) 39(1):71-81. doi:10.1002/hed.24534

8. Sjogren EV, Langeveld TP, Baatenburg de Jong RJ. Clinical outcome of T1 glottic carcinoma since the introduction of endoscopic $\mathrm{CO}_{2}$ laser surgery as treatment option. Head Neck (2008) 30(9):1167-74. doi:10.1002/hed.20852

9. Motta G, Esposito E, Motta S, Tartaro G, Testa D. $\mathrm{CO}_{2}$ laser surgery in the treatment of glottic cancer. Head Neck (2005) 27(8):733. doi:10.1002/hed.20135

10. Meulemans J, Delaere P, Nuyts S, Clement PM, Hermans R, Vander Poorten V. Salvage transoral laser microsurgery for radiorecurrent laryngeal cancer: indications, limits, and outcomes. Curr Otorhinolaryngol Rep (2017) 5(1):83-91. doi:10.1007/s40136-017-0143-7

11. Motamed M, Laccourreye O, Bradley PJ. Salvage conservation laryngeal surgery after irradiation failure for early laryngeal cancer. Laryngoscope (2006) 116(3):451-5. doi:10.1097/01.MLG.0000199591.92336.06

12. Ramakrishnan Y, Drinnan M, Kwong FN, Grant DG, Mehanna H, Jones T, et al. Oncologic outcomes of transoral laser microsurgery for radiorecurrent laryngeal carcinoma: a systematic review and meta-analysis of Englishlanguage literature. Head Neck (2014) 36(2):280-5. doi:10.1002/hed.23291

13. Sobin LH, Compton CC. TNM seventh edition: what's new, what's changed: communication from the International Union Against Cancer and the American Joint Committee on Cancer. Cancer (2010) 116(22):5336-9. doi:10.1002/ cncr.25537

14. Remacle M, Van Haverbeke C, Eckel H, Bradley P, Chevalier D, Djukic V, et al. Proposal for revision of the European Laryngological Society classification of endoscopic cordectomies. Eur Arch Otorhinolaryngol (2007) 264(5):499-504. doi:10.1007/s00405-007-0304-2

15. Remacle M, Eckel HE, Antonelli A, Brasnu D, Chevalier D, Friedrich G, et al. Endoscopic cordectomy. A proposal for a classification by the Working Committee, European Laryngological Society. Eur Arch Otorhinolaryngol (2000) 257(4):227-31. doi:10.1007/s004050050228

16. Steiner W, Vogt P, Ambrosch P, Kron M. Transoral carbon dioxide laser microsurgery for recurrent glottic carcinoma after radiotherapy. Head Neck (2004) 26(6):477-84. doi:10.1002/hed.20009 consent for retrospective studies with anonymized data is not required according to Belgian law.

\section{AUTHOR CONTRIBUTIONS}

JM: data quality control, data analysis (statistics), drafting manuscript, and review of manuscript. JB: data collection, data analysis (statistics), and drafting manuscript. PD: drafting manuscript and review of manuscript. VP: data quality control, drafting manuscript, and review of manuscript.

17. Digonnet A, Hamoir M, Andry G, Haigentz M Jr, Takes RP, Silver CE, et al Post-therapeutic surveillance strategies in head and neck squamous cell carcinoma. Eur Arch Otorhinolaryngol (2013) 270(5):1569-80. doi:10.1007/ s00405-012-2172-7

18. Warren S, Gates O. Multiple primary malignant tumors: a survey of the literature and a statistical study. Am J Cancer (1932) 16:1358-414.

19. Morris LG, Sikora AG, Patel SG, Hayes RB, Ganly I. Second primary cancers after an index head and neck cancer: subsite-specific trends in the era of human papillomavirus-associated oropharyngeal cancer. J Clin Oncol (2011) 29(6):739-46. doi:10.1200/JCO.2010.31.8311

20. Curtis RE, Ries LA. Methods. In: Curtis RE, Freedman DM, Ron E, editors. New Malignancies Among Cancer Survivors: SEER Cancer Registries, 1973-2000. Bethesda, MD: National Cancer Institute (2006). p. 9-14.

21. Schrijvers ML, van Riel EL, Langendijk JA, Dikkers FG, Schuuring E, van der Wal JE, et al. Higher laryngeal preservation rate after $\mathrm{CO}_{2}$ laser surgery compared with radiotherapy in Tla glottic laryngeal carcinoma. Head Neck (2009) 31(6):759-64. doi:10.1002/hed.21027

22. Sjögren EV. Transoral laser microsurgery in early glottic lesions. Curr Otorhinolaryngol Rep (2017) 5(1):56-68. doi:10.1007/s40136-017-0148-2

23. Outzen KE, Illum P. $\mathrm{CO}_{2}$-laser therapy for carcinoma of the larynx. J Laryngol Otol (1995) 109(2):111-3. doi:10.1017/S0022215100129421

24. Quer M, Leon X, Orus C, Venegas P, Lopez M, Burgues J. Endoscopic laser surgery in the treatment of radiation failure of early laryngeal carcinoma. Head Neck (2000) 22(5):520-3. doi:10.1002/1097-0347(200008)22:5<520:: AID-HED13>3.0.CO;2-K

25. de Gier HH, Knegt PP, de Boer MF, Meeuwis CA, van der Velden LA, Kerrebijn JD. $\mathrm{CO}_{2}$-laser treatment of recurrent glottic carcinoma. Head Neck (2001) 23(3): 177-80.doi:10.1002/1097-0347(200103)23:3<177::AID-HED1015>3.0.CO;2-8

26. Pukander J, Kerala J, Makitie A, Hyrynkangas K, Virtaniemi J, Grenman R. Endoscopic laser surgery for laryngeal cancer. Eur Arch Otorhinolaryngol (2001) 258(5):236-9. doi:10.1007/s004050100349

27. Puxeddu R, Piazza C, Mensi MC, Ledda GP, Argiolas F, Peretti G. Carbon dioxide laser salvage surgery after radiotherapy failure in T1 and T2 glottic carcinoma. Otolaryngol Head Neck Surg (2004) 130(1):84-8. doi:10.1016/j. otohns.2003.07.002

28. Ansarin M, Planicka M, Rotundo S, Santoro L, Zurlo V, Maffini F, et al. Endoscopic carbon dioxide laser surgery for glottic cancer recurrence after radiotherapy: oncological results. Arch Otolaryngol Head Neck Surg (2007) 133(12):1193-7. doi:10.1001/archotol.133.12.1193

29. Piazza C, Peretti G, Cattaneo A, Garrubba F, De Zinis LO, Nicolai P. Salvage surgery after radiotherapy for laryngeal cancer: from endoscopic resections to open-neck partial and total laryngectomies. Arch Otolaryngol Head Neck Surg (2007) 133(10):1037-43. doi:10.1001/archotol.133.10.1037

30. Grant DG, Salassa JR, Hinni ML, Pearson BW, Hayden RE, Perry WC. Transoral laser microsurgery for recurrent laryngeal and pharyngeal cancer. Otolaryngol Head Neck Surg (2008) 138(5):606-13. doi:10.1016/j.otohns.2007.12.046

31. Roedel RM, Matthias C, Wolff HA, Schindler P, Aydin T, Christiansen H. Transoral laser microsurgery for recurrence after primary radiotherapy of early glottic cancer. Auris Nasus Larynx (2010) 37(4):474-81. doi:10.1016/j.anl. 2009.11.004

32. Han YJ, Lee HS, Kim SW, Hong JC, Kim ST, Park HS, et al. Transoral laser microsurgery of recurrent early glottic cancer after radiation therapy: clinical feasibility and limitations. Ann Otol Rhinol Laryngol (2012) 121(6):375-82. doi:10.1177/000348941212100603 
33. Fiz I, Koelmel JC, Sittel C. Nature and role of surgical margins in transoral laser microsurgery for early and intermediate glottic cancer. Curr Opin Otolaryngol Head Neck Surg (2018) 26(2):78-83. doi:10.1097/MOO.0000000000000446

34. Fiz I, Mazzola F, Fiz F, Marchi F, Filauro M, Paderno A, et al. Impact of close and positive margins in transoral laser microsurgery for Tis-T2 glottic cancer. Front Oncol (2017) 7:245. doi:10.3389/fonc.2017.00245

35. Murray CE, Cooper L, Handa KK, MacLeod T, MacKenzie K. A technique for the orientation of endoscopically resected laryngeal lesions. Clin Otolaryngol (2007) 32(3):201-3. doi:10.1111/j.1365-2273.2007.01337.x

36. Robertson S, Cooper L, McPhaden A, MacKenzie K. Refining the 'cucumber' technique for laryngeal biopsy. J Laryngol Otol (2011) 125(6):626-9. doi:10.1017/S0022215111000119

37. Chone CT, Yonehara E, Martins JE, Altemani A, Crespo AN. Importance of anterior commissure in recurrence of early glottic cancer after laser endoscopic resection. Arch Otolaryngol Head Neck Surg (2007) 133(9):882-7. doi:10.1001/ archotol.133.9.882
38. Peretti G, Piazza C, Mora F, Garofolo S, Guastini L. Reasonable limits for transoral laser microsurgery in laryngeal cancer. Curr Opin Otolaryngol Head Neck Surg (2016) 24(2):135-9. doi:10.1097/MOO.0000000000000240

39. Del Bon F, Piazza C, Mangili S, Redaelli De Zinis LO, Nicolai P, Peretti G. Transoral laser surgery for recurrent glottic cancer after radiotherapy: oncologic and functional outcomes. Acta Otorhinolaryngol Ital (2012) 32(4):229-37.

Conflict of Interest Statement: The authors do not have any potential conflict of interest to declare in relation with the content of this article.

Copyright (C) 2018 Meulemans, Bijnens, Delaere and Vander Poorten. This is an openaccess article distributed under the terms of the Creative Commons Attribution License (CC BY). The use, distribution or reproduction in other forums is permitted, provided the original author(s) and the copyright owner are credited and that the original publication in this journal is cited, in accordance with accepted academic practice. No use, distribution or reproduction is permitted which does not comply with these terms. 


\title{
Three-Dimensional Map of Isoprognostic Zones in Glottic Cancer Treated by Transoral Laser Microsurgery as a Unimodal Treatment Strategy
}

\author{
Cesare Piazza ${ }^{1 *}$, Marta Filauro ${ }^{2}$, Alberto Paderno ${ }^{1,3}$, Filippo Marchi ${ }^{2}$, Pietro Perotti ${ }^{1}$, \\ Riccardo Morello ${ }^{3}$, Stefano Taboni ${ }^{3}$, Giampiero Parrinello ${ }^{2}$, Fabiola Incandela ${ }^{1,2}$, \\ Andrea landelli ${ }^{2}$, Francesco Missale $^{2}$ and Giorgio Peretti ${ }^{2}$
}

\begin{abstract}
'Department of Otorhinolaryngology - Head and Neck Surgery, Fondazione IRCCS, National Cancer Institute of Milan, University of Milan, Milan, Italy, ${ }^{2}$ Department of Otorhinolaryngology - Head and Neck Surgery, University of Genoa, Genoa, Italy, ${ }^{3}$ Department of Otorhinolaryngology - Head and Neck Surgery, University of Brescia, Brescia, Italy
\end{abstract}

OPEN ACCESS

Edited by:

David I. Rosenthal, University of Texas MD Anderson Cancer Center, United States

Reviewed by:

Sebastien Vergez, Institut universitaire du Cancer de Toulouse Oncopole, France Jeroen Meulemans, University Hospitals Leuven, Belgium

*Correspondence:

Cesare Piazza cesare.piazza@istitutotumori.mi.it

Specialty section: This article was submitted to Head and Neck Cancer. a section of the journal

Frontiers in Oncology

Received: 05 March 2018 Accepted: 04 May 2018 Published: 22 May 2018

Citation:

Piazza C, Filauro M, Paderno A, Marchi F, Perotti P, Morello R, Taboni S, Parrinello G, Incandela F, landelli A, Missale F and Peretti G

(2018) Three-Dimensional Map of Isoprognostic Zones in Glottic Cancer

Treated by Transoral Laser

Microsurgery as a Unimodal Treatment Strategy.

Front. Oncol. 8:175.

doi: 10.3389/fonc.2018.00175
Introduction: The Union for International Cancer Control-American Joint Committee on Cancer TNM staging system for glottic squamous cell carcinoma (SCC) includes different types of lesions defined by the involvement of specific subsites in each T category. Our study aims to identify different subcategories according to tumor local extension and determine oncologic outcomes after treatment by transoral laser microsurgery (TLM) alone.

Methods: We retrospectively evaluated 410 patients affected by previously untreated pT1-pT3 glottic SCC treated by TLM alone from January 2005 to December 2015 at the Departments of Otorhinolaryngology_Head and Neck Surgery, Universities of Genoa and Brescia, Italy. All patients had at least 2 years of follow-up. Clinical, radiological, surgical, and histopathological data were reviewed and tumors divided into six subcategories: I, pT1a not involving the anterior commissure (AC); II, pT1b involving the AC; III, pT2 extending superficially to the supraglottis or the subglottis; IV, pT2 infiltrating the vocal muscle; V, pT3 involving the anterior paraglottic space; VI, pT2 or pT3 with vertical extension across the $\mathrm{AC}$ with/without involvement of the pre-epiglottic space. Recurrence-free survival (RFS), local control with laser alone (LCL), and organ preservation (OP) were defined as the primary oncologic outcomes.

Results: The 2, 5, and 10-year RFS for the entire series were 85.7, 80.3, and 73.8\%, LCL rates 93.8, 92.1, and 89.6\%, and OP rates 96.8, 95.9, and 93.5\%, respectively. However, when comparing the rates of RFS, LCL, and OP for each subcategory, important differences emerged. In particular, subcategories $V$ and $V I$ showed a significantly increased risk of local recurrence [hazard ratio $(H R)=9.2$ and 13.3, respectively]. These subcategories also had a significantly reduced probability to achieve LCL (HR: 73.6 and 93.5, respectively) and OP (HR: 6.4 and 8.1, respectively).

Conclusion: The present classification in subcategories allows introducing the concept of a three-dimensional map of isoprognostic zones in glottic SCC treated by TLM alone as a useful tool in its management by a multidisciplinary tumor board.

Keywords: transoral laser microsurgery, laryngeal cancer, glottic cancer, carbon dioxide laser, prognosis, oncologic outcomes 


\section{INTRODUCTION}

Modern literature is replete of clear-cut evidence in favor of the good oncologic and functional outcomes of transoral laser microsurgery (TLM) for treatment of T1-T2 and selected T3 glottic squamous cell carcinomas (SCC) (1-14). Accurate and tailored patient selection, application of a multi-step diagnostic work-up based on pre- and intraoperative endoscopy, surgically oriented imaging assessment by dedicated head and neck radiologists, use of a state-of-the-art laser technology, and knowledge of pathways of spreading and recurrence have allowed clinicians to obtain, in large-volume oncologic centers, outstanding and reproducible results. However, even the $8^{\text {th }}$ Edition of the TNM staging system (15) is definitively too simplistic to precisely define the different possible extensions of glottic tumors (especially when dealing with $\mathrm{T} 2$ and $\mathrm{T} 3$ lesions), thus limiting a proper patient and interdisciplinary counseling. In fact, these $\mathrm{T}$ categories may differ according to the involvement of various glottic subsites, superficial spreading to the supraglottis or subglottis, deep extension toward visceral compartments [paraglottic (PGS) and pre-epiglottic spaces (PES)], vocal cord/arytenoid mobility, and infiltration of the cartilaginous framework.

Too frequently, even in well-structured multidisciplinary boards, the "T-N categories criterion" is the only one used to define a lesion and, therefore, to choose among the most appropriate therapeutic strategies. Indeed, our experience $(4,5,9)$, as well as those of others $(6-8)$, clearly shows that profound differences exist among tumors usually grouped together under the same T category but, in reality, presenting very different surgical challenges, oncological prognosis, and possible functional sequelae. The process of subdividing each $\mathrm{T}$ category in a number of more homogeneous subgroup of lesions should help in communicating and sharing therapeutic outcomes to be achieved in every single case scenario, thus making a custom tailored approach for evaluation and treatment of a specific tumor more feasible.

The present study introduces a new conceptual tool that we have defined "three-dimensional (3D) map of isoprognostic zones," which allows for the definition, for each therapeutic strategy, of the oncologic outcomes that are reasonably obtainable for each specific $\mathrm{T}$ subcategory, more precisely than previously obtained by the simple use of TNM stratification alone. Herein, we embraced such a subcategorization to depict an isoprognostic zones map related to the use of TLM as a unimodal treatment strategy for management of early-intermediate glottic SCCs. However, the same process could be extended to other $\mathrm{T}$ and $\mathrm{N}$ categories, laryngeal subsites, or head and neck sites, as well as to other surgical and non-surgical treatment modalities, taken alone or in various combinations.

\footnotetext{
Abbreviations: 3D, three-dimensional; UICC, Union for International Cancer Control; AJCC, American Joint Committee on Cancer; SCC, squamous cell carcinoma; TLM, transoral laser microsurgery; AC, anterior commissure; RFS, recurrence-free survival; LCL, local control with laser alone; OP, organ preservation; HR, hazard ratio; PGS, paraglottic space; PES, pre-epiglottic space; RT, radiotherapy; CRT, chemoradiotherapy; OPHL, open partial horizontal laryngectomy; WL, white light; NBI, narrow band imaging; CT, computed tomography; MR, magnetic resonance.
}

The aim of the present study is to retrospectively stratify in 6 subcategories, defining an equal number of isoprognostic zones, a cohort of $410 \mathrm{~T} 1, \mathrm{~T} 2$, and selected T3 glottic SCCs according to location and extension, describe different patterns of growth and possible pathways of recurrence, and define the role and limits of TLM as a single treatment modality in terms of recurrence-free survival (RFS), local control with laser alone (LCL), and organ preservation $(\mathrm{OP})$ rates for each subcategory.

\section{MATERIALS AND METHODS}

We retrospectively evaluated 410 patients $(371$ males and 39 females) affected by previously untreated T1-T3 glottic SCC managed by TLM alone from January 2005 to December 2015 at the Departments of Otorhinolaryngology-Head and Neck Surgery, Universities of Genoa and Brescia, Italy. Patients age ranged from 31 to 94 years (median, 68), and all had at least 2 years of follow-up (range, 24-120 months; median, 72). Inclusion criteria were glottic pT1, pT2, and selected pT3 SCC with evidence of limited anterior PGS and/or PES involvement and adequate laryngeal exposure preoperatively assessed by the Laryngoscore (16). Patients affected by T3 glottic SCC with minimal laryngeal framework invasion or arytenoid fixation due to posterior PGS involvement and those previously treated elsewhere with TLM, radiotherapy (RT), chemoradiotherapy (CRT), or open partial horizontal laryngectomies (OPHL) were excluded from the present study.

Preoperative flexible videolaryngoscopy assessed superficial tumor margins under white light (WL) and narrow band imaging (NBI, Olympus Medical System Corporation, Tokyo, Japan). Deep neoplastic extension was evaluated using contrast enhanced imaging [computed tomography or magnetic resonance (MR)] performed by dedicated head and neck radiologists. Neck ultrasound with or without fine needle aspiration cytology was routinely performed in lesions with significant supraglottic extension or those defined as glottic cT3. Intraoperative rigid endoscopy with $0^{\circ}, 30^{\circ}$, and $70^{\circ}$ telescopes under WL and NBI was accomplished during microlaryngoscopy to obtain better definition of superficial surgical margins (17). All patients underwent TLM using previously described surgical techniques and instrumentation $(9,16)$. Patients with clinically negative neck nodes received purely local treatment by TLM. Four patients with clinically positive neck disease underwent unilateral selective (levels II-V) neck dissection. Transoral re-excision was accomplished in case of deep or more than one superficial positive margins. We also excluded patients who received adjuvant treatments (RT and/or CRT) due to the presence of persistent tumor after re-excision, perineural invasion, angioembolization, multiple positive lymph nodes, or extracapsular spread. We retrospectively reviewed clinical, radiological, surgical, and histopathological data, and divided tumors accordingly into six subcategories: I, pT1a not involving the anterior commissure (AC); II, pT1b involving the AC or both vocal folds; III, pT2 extending superficially to the supraglottis or the subglottis; IV, pT2 infiltrating the vocal muscle (VM); V, pT3 involving the anterior PGS; VI, pT2 or pT3 with trans-AC extension with or without involvement of the PES. 
All tumors were restaged according to the eighth edition of the Union for International Cancer Control-American Joint Committee on Cancer TNM staging system (15). At the time of diagnosis, the cT categories were as follows: 284 cT1a-b, 84 cT2, and 42 cT3. The pT stage and the ensuing division in subcategories were 211 pT1a (subcategory I); 61 pT1b (subcategory II); 45 pT2 (subcategory III); 41 pT2 (subcategory IV); 40 pT3 (subcategory V); and 12 pT2-pT3 (subcategory VI).

No approval from the Ethics Committee for this study was deemed necessary at our Institutions after a formal request to the appropriate parties. Each patient signed an informed consent for treatment of personal data for scientific purposes before enrollment.

\section{Statistical Analysis}

Statistical analysis was carried out using STATA 13 software (StataCorp. 2013. Stata Statistical Software: Release 13. College Station, TX, USA: StataCorp LP). 2-, 5-, and 10-year RFS, LCL, and OP rates were evaluated by the Kaplan-Meier product limit estimate. Subcategories were first individually compared among each other by the log-rang test (I vs. II, II vs. III, III vs. IV, IV vs. $\mathrm{V}$, and V vs. VI) to identify major differences in terms of RFS, LCL, and OP. Subcategory I has been considered the reference point [hazard ratio $(\mathrm{HR})=1$ ] when evaluating RFS and LCL. Conversely, the reference point for OP was subcategory II, due to the lack of events (total laryngectomies) in subcategory I. Furthermore, a Cox proportional hazard regression model was employed to quantify the stratification potential of subcategories (considered as a categorical variable) and to obtain the related HR for RFS, LCL, and OP. No further variable was included in the regression model, since a different distribution of risk factors was not considered as a confounding element, but as one of the bases for the subcategory stratification itself. Comparisons between survival curves were carried out using the log-rank test. The final time-point for RFS was the date of the first recurrence (patients who died without any recurrence were considered as censored). The final time-point for LCL was the date of any further open or non-surgical treatment for relapse. The final time-point for OP was the date of total laryngectomy (patients who died without a previous total laryngectomy were considered as censored).

\section{RESULTS}

Fifty-nine (14.4\%) patients had recurrences. Forty-nine had a local recurrence: 13 in subcategory I (6\% of patients in such subcategory), 11 in subcategory II (18\%), 7 in subcategory III (15\%), 6 in subcategory IV (15\%), 8 in subcategory V (20\%), and 4 in subcategory VI (33\%). Nine patients experienced loco-regional recurrence: 1 in subcategory III (2\%), 3 in subcategory IV (7\%), 3 in subcategory V (8\%), and 2 in subcategory VI (17\%). Only one patient in subcategory $\mathrm{V}(2 \%)$ had an exclusively regional recurrence. Salvage treatment was represented by TLM in 40 patients, OPHL with uni- or bilateral selective neck dissection in 6, total laryngectomy with uni- or bilateral neck dissection in 13 (4 of them followed by CRT), RT alone in 1, and chemotherapy alone in the only patient with neck failure. One patient received best supportive care due to refusal of any further treatment. At the last follow-up (December 2017), 57 patients had died: 8 for disease progression, and 49 for other causes. Four patients were alive with disease, while in the remaining 349 there was no evidence of disease.

2-, 5-, and 10-year RFS rates for the entire series were 85.7, 80.3 , and $73.8 \%$, LCL rates were $93.8,92.1$, and $89.6 \%$, and OP rates were 96.8, 95.9, and 93.5\%, respectively. RFS, LCL, and OP rates according to each pT subcategory are detailed in Table 1 and Figures 1-3. Progressively comparing the estimates of the hazard functions of each subcategory with the following one, we evidenced a significant difference at the log-rank test between subcategory I and II $(p=0.004)$, as well as IV and V $(p=0.050)$ when considering LCL. The significant difference between subcategory I and II was also confirmed when considering RFS and OP (both $p<0.001$ ).

In the Cox proportional hazards regression analysis, according to our patient stratification, subcategories V and VI showed an approximately 10 -fold increase in the risk of local recurrence, with an hazard ratio (HR) of 9.2 and 13.3, respectively $(p<0.001)$. In addition, the same subcategories had a greatly reduced probability to achieve LCL $(\mathrm{HR}=73.6$ and 93.5, respectively; $p<0.001)$ and $\mathrm{OP}(\mathrm{HR}=6.4$ and 8.1 , respectively; $p<0.01)$. HRs concerning RFS, LCL, and OP for each subcategory are detailed in Table 2.

Three-dimensional maps of the isoprognostic zones for glottic SCCs treated by TLM alone are depicted in Figures 4-6.

\section{DISCUSSION}

The introduction of a novel conceptual tool such as the present that we defined "3D map of isoprognostic zones" tries to overcome, in our opinion, one of the most limiting factors in the contemporary literature concerning TLM for laryngeal SCC. In fact, oncologic outcomes of such a technique (as well

TABLE 1 | Recurrence-free survival (RFS), local control with laser alone (LCL), and organ preservation (OP) at 2, 5, and 10 years for each subcategory.

\begin{tabular}{|c|c|c|c|c|c|c|c|c|c|}
\hline & \multicolumn{3}{|c|}{ RFS } & \multicolumn{3}{|c|}{ LCL } & \multicolumn{3}{|c|}{ OP } \\
\hline Overall series & 85.7 & 80.3 & 73.8 & 93.8 & 92.1 & 89.6 & 96.8 & 95.9 & 93.5 \\
\hline Subcategory II & 82.1 & 68.0 & - & 100 & 89.4 & 74.5 & 97.6 & 90.1 & 77.2 \\
\hline Subcategory III & 85.5 & 81.6 & 68.0 & 90.7 & 90.7 & 90.7 & 95.1 & 95.1 & 95.1 \\
\hline Subcategory IV & 77.1 & 68.1 & 68.1 & 86.1 & 86.1 & 86.1 & 94.4 & 94.4 & 94.4 \\
\hline
\end{tabular}




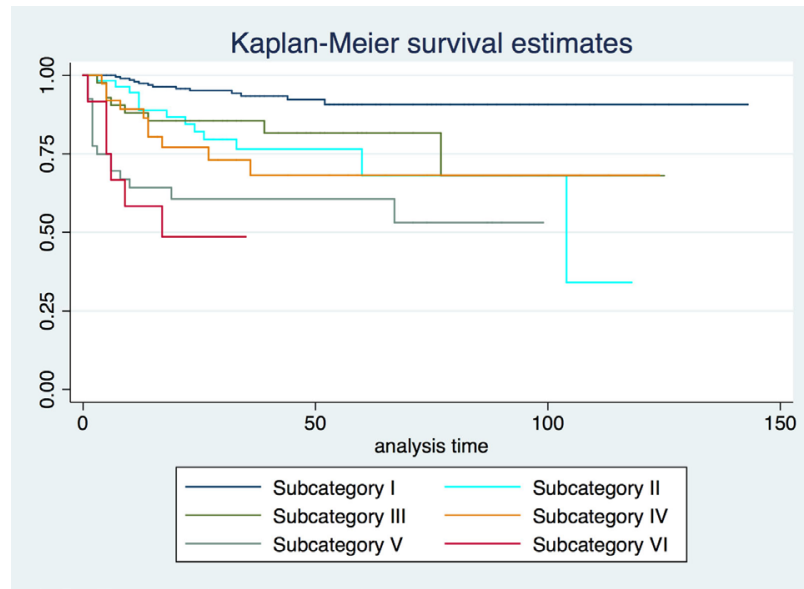

FIGURE 1 | Recurrence-free survival Kaplan-Meier curves for each subcategory.

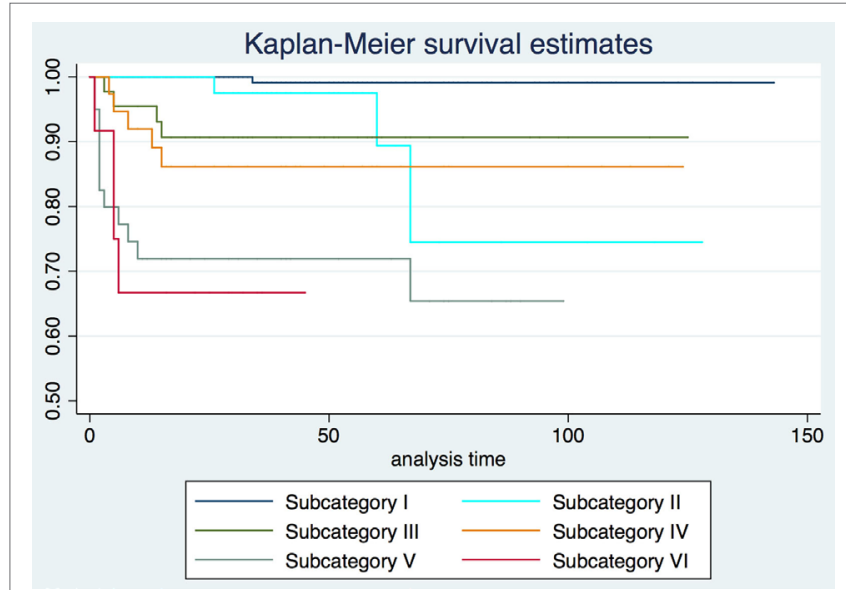

FIGURE 2 | Local control with laser alone Kaplan-Meier curves for each subcategory.

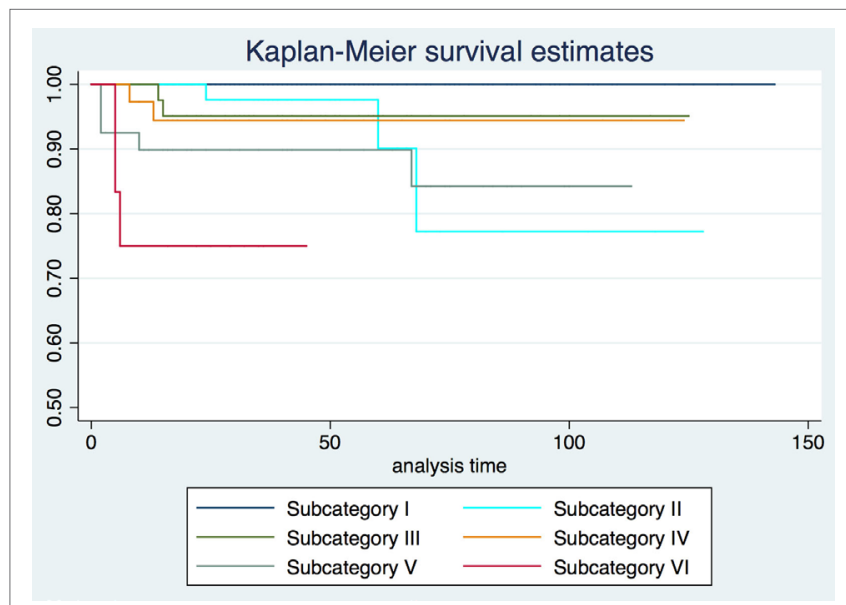

FIGURE 3 | Organ preservation Kaplan-Meier curves for each subcategory. as those of many other surgical and non-surgical therapeutic options) are frequently reported on the simple basis of $\mathrm{T}$ category, if not stage, placing a number of different lesions such as T1a, T1b, T2, and limited T3 under the same umbrella term of "early-intermediate" tumors. The ensuing overall outcomes may, therefore, seem quite encouraging even though, in reality, a much more differentiated scenario can be described when one specifically peruses the $\mathrm{T}$ category and, especially, applies more detailed subcategorization, as herein reported, that is able to consider specific issues. Such an approach directly derives from the daily use of an intraoperative microscopic view of such lesions, coupled with systematic application of modern biologic endoscopy techniques, and high-resolution radiologic imaging. All these tools are more articulated and time-consuming but, nonetheless, provide a comprehensive description of the different possible pathways of spread of glottic SCCs. In fact, superficial as well as deep progression of the lesion may harbor different challenges in terms of local control and OP. In particular, the present data confirm those already published by our group $(5,9,18,19)$ and together form the basis of the conceptualization attempted by the $3 \mathrm{D}$ map of isoprognostic zones herein described.

Our results confirm both the outstanding outcomes for the entire cohort in terms of RFS, LCL, and OP rates obtained by TLM alone and the need to analyze each subcategory to better highlight the real value of a treatment in each isoprognostic zone. Indeed, the present survival curves are effectively stratified according to our proposal of subcategorization. The HR for RFS was 4.5, 9.2, and 13.3 times significantly higher in subcategories IV, V, and VI, respectively, thus emphasizing that the vertical extension across the $\mathrm{AC}$ with/without involvement of the PES should be considered as a real weakness for the TLM approach. Such a crucial issue is also confirmed by the reduced LCL and the higher need for salvage total laryngectomy in subcategory VI compared with other isoprognostic zones of the $3 \mathrm{D}$ map.

In our opinion, subcategory VI has the worst outcome in the present series since transglottic SCCs tend to spread from the PES upwards into the supraglottis and hence outside the larynx due to the absence of a significant anatomical barrier in this area, or directly through the crico-thyroid membrane at the subglottic level. Moreover, the tangential transoral microscopic visualization of the supra- and subcommissural areas may further penalize adequate surgical treatment of tumors deeply involving these visceral compartments.

When sequentially comparing the single subcategories, it is possible to observe a significant shift in LCL from subcategory I to II-III, as well as from IV to V-VI. This may be seen as the passage from a superficially confined neoplasm (subcategory I) to a superficially spreading tumor (subcategories II-III), finally shifting to a deeply infiltrating lesion involving the laryngeal visceral spaces (subcategories V-VI).

Still, our results, in line with the literature, highlight how patients in the IV subcategory have a worse outcome compared to the III subcategory, even if both belong to the pT2 category, while those included in subcategory $\mathrm{V}$ for deep extension to the anterior PGS (limited pT3 lying in front of a plane tangential to 
TABLE 2 | Cox analysis between subcategories of recurrence-free survival (RFS), local control with laser alone (LCL), and organ preservation (OP).

\begin{tabular}{|c|c|c|c|c|c|c|c|c|c|}
\hline & \multicolumn{3}{|c|}{ RFS } & \multicolumn{3}{|c|}{ LCL } & \multicolumn{3}{|c|}{ OP } \\
\hline & Hazard ratio (HR) & $95 \% \mathrm{Cl}$ & $p$-Value & HR & $95 \% \mathrm{Cl}$ & $p$-Value & HR & $95 \% \mathrm{Cl}$ & $p$-Value \\
\hline Subcategory I & 1.0 & - & - & 1.0 & - & - & - & - & - \\
\hline Subcategory II & 4.0 & 1.86-8.68 & $<0.001$ & 11.4 & $1.18-109.40$ & 0.035 & 1.0 & - & - \\
\hline Subcategory III & 3.1 & $1.28-7.50$ & 0.012 & 18.8 & $2.11-168.50$ & 0.009 & 1.7 & $0.37-7.41$ & 0.508 \\
\hline Subcategory IV & 4.5 & 1.98-10.33 & $<0.001$ & 28.2 & $3.29-241.09$ & 0.002 & 2.5 & $0.59-10.31$ & 0.218 \\
\hline Subcategory V & 9.2 & $4.45-19.33$ & $<0.001$ & 73.6 & $9.55-566.86$ & $<0.001$ & 6.4 & $1.80-22.91$ & 0.004 \\
\hline Subcategory VI & 13.3 & $5.01-35.27$ & $<0.001$ & 93.5 & $10.41-840.35$ & $<0.001$ & 8.1 & $1.80-36.18$ & 0.006 \\
\hline
\end{tabular}

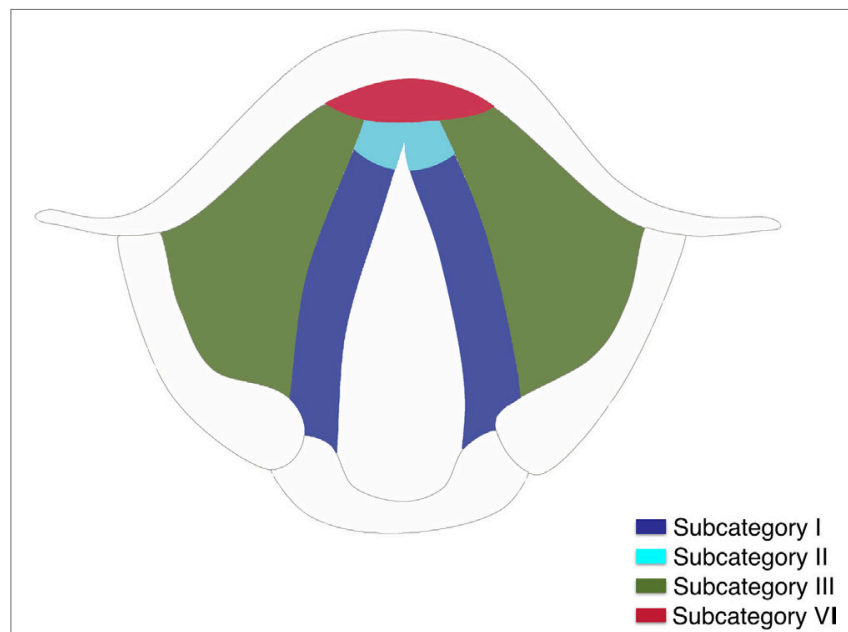

FIGURE 4 | Three-dimensional map of the isoprognostic zones I, II, III, and $\mathrm{VI}$, view from above.

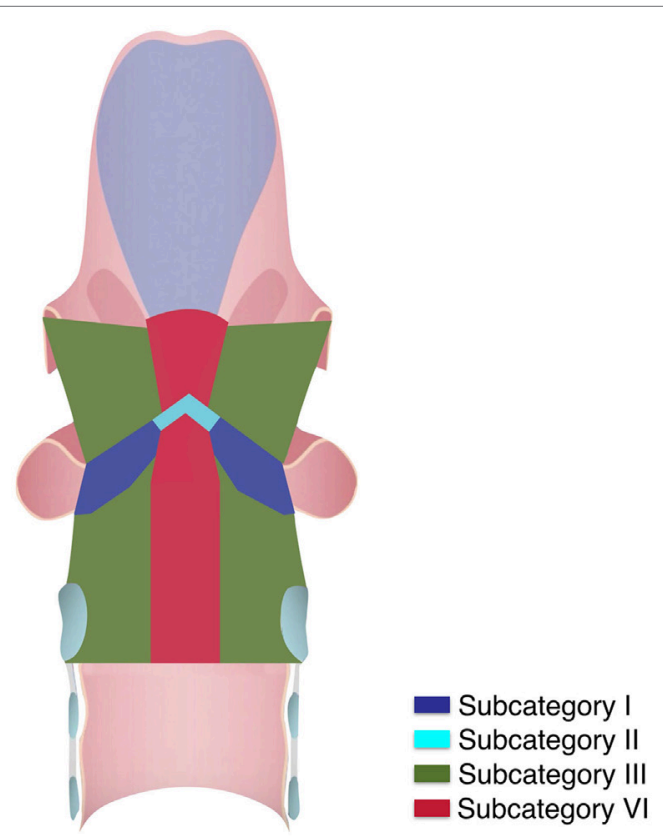

FIGURE 5 | Three-dimensional map of the isoprognostic zones I, II, III, and $\mathrm{VI}$, view from inside.

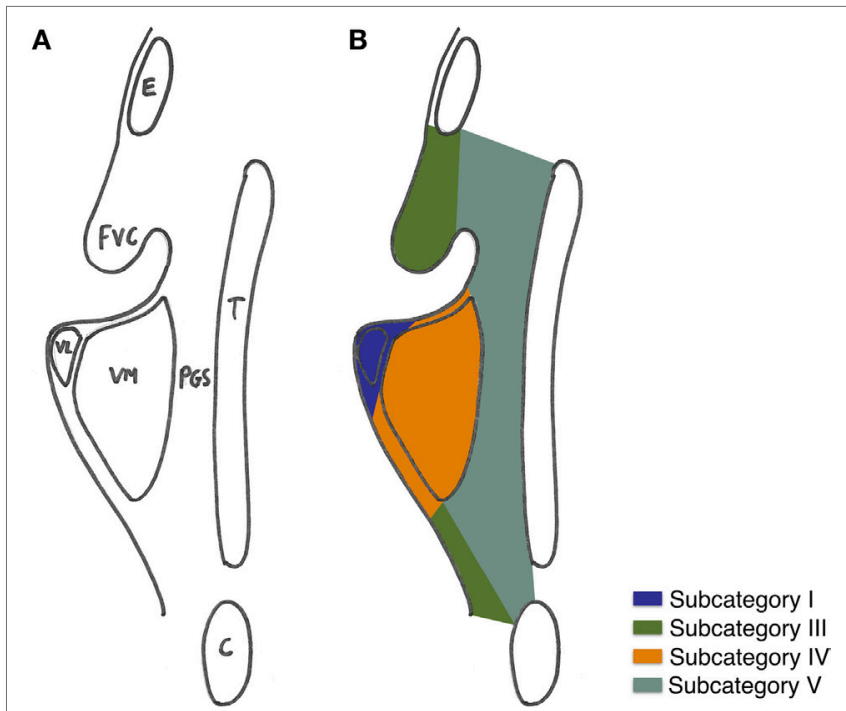

FIGURE 6 | (A) Coronal view of the right hemilarynx, anterior half (E, epiglottis; FVC, false vocal cord; VL, vocal ligament; VM, vocal muscle; PGS, anterior paraglottic space; T, thyroid cartilage; C, cricoid) and (B) three-dimensional map of the isoprognostic zones I, III, IV, and V, coronal view of the right hemilarynx, anterior half.

the arytenoid vocal process and perpendicular to the ipsilateral thyroid lamina) have comparable outcomes with other traditional therapeutic options (20-22).

In light of this, subcategories IV, V, and VI represent the highest-risk group of patients to be managed by TLM alone. Thus, as already demonstrated in previous reports (23-25), they merit planned radiological examination (better with MR) even in the absence of clinical and endoscopic doubts during follow-up, due to their well-known propensity to recur submucosally.

In conclusion, we can assume that the propensity to use the term "early-intermediate stage" to describe a wide spectrum of diseases ranging from lesions limited to one vocal cord to those with a trans-AC extension as well VM infiltration or invasion of the anterior PGS and/or PES, may risk erroneous inclusion in a given prognostic category, with subsequent application of suboptimal treatment strategies (26-28). Ideal oncological and functional outcomes require accurate and comprehensive preand intraoperative diagnostic work-up, specialized skills, and expertise. Poor judgment will increase the risk of recurrence or the need for multimodal treatments (with associated increased 
toxicity and sequelae) to achieve disease control and functional preservation.

We must not forget, however, that the isoprognostic zones presented herein are strictly related and influenced by the specific therapeutic approach chosen, i.e., in the present series, by TLM. However, this should not limit their application to other treatment modalities and, even more interestingly, could fuel a stimulating comparison between them, especially in the context of a multidisciplinary tumor board. Our ideal target in the future would be, therefore, to be able to precisely choose, for each lesion in a given patient, the most adequate therapeutic approach, in order to ensure the highest oncological success with the lowest impact in terms of morbidity and costs.

\section{REFERENCES}

1. McMullen CP, Smith RV. Treatment/comparative therapeutics: cancer of the larynx and hypopharynx. Surg Oncol Clin N Am (2015) 24:521-45. doi:10.1016/j.soc.2015.03.013

2. Breda E, Catarino R, Monteiro E. Transoral laser microsurgery for laryngeal carcinoma: survival analysis in a hospital-based population. Head Neck (2015) 37:1181-6. doi:10.1002/hed.23728

3. Hakeem AH, Tubachi J, Pradhan SA. Significance of anterior commissure involvement in early glottic squamous cell carcinoma treated with transoral $\mathrm{CO}_{2}$ laser microsurgery. Laryngoscope (2013) 123:1912-7. doi:10.1002/ lary.24012

4. Peretti G, Piazza C, Mora F, Garofolo S, Guastini L. Reasonable limits for transoral laser microsurgery in laryngeal cancer. Curr Opin Otolaryngol Head Neck Surg (2016) 24:135-9. doi:10.1097/MOO.0000000000000240

5. Peretti G, Piazza C, Mensi MC, Magnoni L, Bolzoni A. Endoscopic treatment of cT2 glottic carcinoma: prognostic impact of different pT subcategories. Ann Otol Rhinol Laryngol (2005) 114:579-86. doi:10.1177/000348940511400801

6. Vilaseca I, Bernal-Sprekelsen M, Blanch JL. Transoral laser microsurgery for T3 laryngeal tumors: prognostic factors. Head Neck (2010) 32:929-38. doi:10.1002/hed.21288

7. Canis M, Ihler F, Martin A, Wolff HA, Matthias C, Steiner W. Results of 226 patients with T3 laryngeal carcinoma after treatment with transoral laser microsurgery. Head Neck (2014) 36:652-9. doi:10.1002/hed.23338

8. Canis M, Martin A, Ihler F, Wolff HA, Kron M, Matthias C, et al. Transoral laser microsurgery in treatment of pT2 and pT3 glottic laryngeal squamous cell carcinoma - results of 391 patients. Head Neck (2014) 36:859-66. doi:10.1002/hed.23389

9. Peretti G, Piazza C, Cocco D, De Benedetto L, Del Bon F, Redaelli De Zinis LO, et al. Transoral $\mathrm{CO}(2)$ laser treatment for $\mathrm{T}(\mathrm{is})-\mathrm{T}(3)$ glottic cancer: the University of Brescia experience on 595 patients. Head Neck (2010) 32:977-83. doi:10.1002/hed.21278

10. FakhryN, Vergez S, Babin E, BaumstarckK, Santini L, Dessi P, et al. Management of surgical margins after endoscopic laser surgery for early glottic cancers: a multicentric evaluation in French-speaking European countries. Eur Arch Otorhinolaryngol (2015) 272:1465-9. doi:10.1007/s00405-015-3547-3

11. Riga M, Chelis L, Danielides V, Vogiatzaki T, Pantazis TL, Pantazis D. Systematic review on T3 laryngeal squamous cell carcinoma; still far from a consensus on the optimal organ preserving treatment. Eur J Surg Oncol (2017) 43:20-31. doi:10.1016/j.ejso.2016.05.030

12. Phillips TJ, Sader C, Brown T, Bullock M, Wilke D, Trites JR, et al. Transoral laser microsurgery versus radiation therapy for early glottic cancer in Canada: cost analysis. JOtolaryngol Head Neck Surg (2009) 38:619-23. doi:10.2310/7070.2009.080254

13. Mandelbaum RS, Abemayor E, Mendelsohn AH. Laryngeal preservation in glottic cancer: a comparison of hospital charges and morbidity among treatment options. Otolaryngol Head Neck Surg (2016) 155:265-73. doi:10.1177/ 0194599816639248

14. Pantazis D, Liapi G, Kostarelos D, Kyriazis G, Pantazis TL, Riga M. Glottic and supraglottic pT3 squamous cell carcinoma: outcomes with transoral laser

\section{ETHICS STATEMENT}

No approval from the Ethics Committee for this study was deemed necessary at our Institutions after a formal request to the appropriate parties. Each patient signed an informed consent for treatment of personal data for scientific purposes before enrollment.

\section{AUTHOR CONTRIBUTIONS}

CP and GP: study design, conceptualization, definitive manuscript draft, and revision; MF, AP, FM, RM, ST, GiPa, FI, AI and FrMi: charts review, data collection, follow-up, and initial manuscript draft; AP and FrMi: statistical analysis; CP: drawings.

microsurgery. Eur Arch Otorhinolaryngol (2015) 272:1983-90. doi:10.1007/ s00405-015-3611-z

15. Brierley JD, Gospodarowicz MK, Wittekind C. TNM Classification of Malignant Tumours. 8th ed. Chichester: John Wiley and Sons (2017). 272 p.

16. Piazza C, Mangili S, Bon FD, Paderno A, Grazioli P, Barbieri D, et al. Preoperative clinical predictors of difficult laryngeal exposure for microlaryngoscopy: the Laryngoscore. Laryngoscope (2014) 124:2561-7. doi:10.1002/lary.24803

17. Garofolo S, Piazza C, Del Bon F, Mangili S, Guastini L, Mora F, et al. Intraoperative Narrow Band Imaging better delineates superficial resection margins during transoral laser microsurgery for early glottic cancer. Ann Otol Rhinol Laryngol (2015) 124:294-8. doi:10.1177/0003489414556082

18. Peretti G, Piazza C, Penco S, Santori G, Del Bon F, Garofolo S, et al. Transoral laser microsurgery as primary treatment for selected $\mathrm{T} 3$ glottic and supraglottic cancers. Head Neck (2016) 38:1107-12. doi:10.1002/hed.24424

19. Peretti G, Piazza C, Bolzoni A, Mensi MC, Rossini M, Parrinello G, et al. Analysis of recurrences in $322 \mathrm{Tis}, \mathrm{T} 1$, or T2 glottic carcinomas treated by carbon dioxide laser. Ann Otol Rhinol Laryngol (2004) 113:853-8. doi:10.1177/000348940411301101

20. Succo G, Crosetti E, Bertolin A, Lucioni M, Caracciolo A, Panetta V, et al. Benefits and drawbacks of open partial horizontal laryngectomies, Part A: early- to intermediate-stage glottic carcinoma. Head Neck (2016) 38(Suppl 1): E333-40. doi:10.1002/hed.23997

21. Succo G, Crosetti E, Bertolin A, Lucioni M, Arrigoni G, Panetta V, et al. Benefits and drawbacks of open partial horizontal laryngectomies, Part B: intermediate and selected advanced stage laryngeal carcinoma. Head Neck (2016) 38(Suppl 1):E649-57. doi:10.1002/hed.24064

22. Yamazaki H, Suzuki G, Nakamura S, Hirano S, Yoshida K, Konishi K, et al. Radiotherapy for locally advanced resectable T3-T4 laryngeal cancer - does laryngeal preservation strategy compromise survival? J Radiat Res (2018) 59:77-90. doi:10.1093/jrr/rrx063

23. Ravanelli M, Farina D, Rizzardi P, Botturi E, Prandolini P, Mangili S, et al. MR with surface coils in the follow-up after endoscopic laser resection for glottic squamous cell carcinoma: feasibility and diagnostic accuracy. Neuroradiology (2013) 55:225-32. doi:10.1007/s00234-012-1128-3

24. Maroldi R, Ravanelli M, Farina D. Magnetic resonance for laryngeal cancer. Curr Opin Otolaryngol Head Neck Surg (2014) 22:131-9. doi:10.1097/ MOO.0000000000000036

25. Marchi F, Piazza C, Ravanelli M, Gaggero G, Parrinello G, Paderno A, et al. Role of imaging in the follow-up of T2-T3 glottic cancer treated by transoral laser microsurgery. Eur Arch Otorhinolaryngol (2017) 274:3679-86. doi:10.1007/s00405-017-4642-4

26. Gorphe P, Blanchard P, Breuskin I, Temam S, Tao Y, Janot F. Vocal fold mobility as the main prognostic factor of treatment outcomes and survival in stage II squamous cell carcinomas of the glottic larynx. J Laryngol Otol (2015) 129:903-9. doi:10.1017/S002221511500184X

27. Warner L, Lee K, Homer JJ. Transoral laser microsurgery versus radiotherapy for T2 glottic squamous cell carcinoma: a systematic review of local control outcomes. Clin Otolaryngol (2017) 42:629-36. doi:10.1111/coa. 12790 
28. McCoul ED, Har-El G. Meta-analysis of impaired vocal cord mobility as a prognostic factor in T2 glottic carcinoma. Arch Otolaryngol Head Neck Surg (2009) 135:479-86. doi:10.1001/archoto.2009.47

Conflict of Interest Statement: The authors declare that the research was conducted in the absence of any commercial or financial relationships that could be construed as a potential conflict of interest.
Copyright (c) 2018 Piazza, Filauro, Paderno, Marchi, Perotti, Morello, Taboni, Parrinello, Incandela, Iandelli, Missale and Peretti. This is an open-access article distributed under the terms of the Creative Commons Attribution License (CC $B Y)$. The use, distribution or reproduction in other forums is permitted, provided the original author(s) and the copyright owner are credited and that the original publication in this journal is cited, in accordance with accepted academic practice. No use, distribution or reproduction is permitted which does not comply with these terms. 


\section{OPEN ACCESS}

Edited by:

Cesare Piazza,

Istituto Nazionale dei Tumori (IRCCS),

Italy

Reviewed by:

Giuseppe Mercante, Istituto Nazionale del Cancro Regina

Elena, Italy

Kenji Okami

Tokai University, Japan

${ }^{\star}$ Correspondence:

Filippo Carta

filippocarta@unica.it

Specialty section:

This article was submitted to

Head and Neck Cancer

a section of the journal

Frontiers in Oncology

Received: 13 April 2018

Accepted: 27 July 2018

Published: 04 September 2018

Citation:

Carta F, Mariani C, Sambiagio GB,

Chuchueva N, Lecis E, Gerosa C and

Puxeddu R (2018) $\mathrm{CO}_{2}$ Transoral Microsurgery for Supraglottic

Squamous Cell Carcinoma

Front. Oncol. 8:321.

doi: $10.3389 /$ fonc. 2018.00321

\section{$\mathrm{CO}_{2}$ Transoral Microsurgery for Supraglottic Squamous Cell Carcinoma}

\author{
Filippo Carta ${ }^{1 *}$, Cinzia Mariani ${ }^{1}$, Giovanni B. Sambiagio ${ }^{2}$, Natalia Chuchueva $^{3}$, \\ Elisa Lecis ${ }^{1}$, Clara Gerosa ${ }^{4}$ and Roberto Puxeddu ${ }^{1}$
}

\begin{abstract}
${ }^{1}$ Unit of Otorhinolaryngology, Department of Surgery, Azienda Ospedaliero-Universitaria di Cagliari, University of Cagliari, Cagliari, Italy, ${ }^{2}$ NHS Lanarkshire, Glasgow, United Kingdom, ${ }^{3}$ I.M. Sechenov First Moscow State Medical University, Moscow, Russia, ${ }^{4}$ Unit of Pathology, Department of Surgery, Azienda Ospedaliero-Universitaria di Cagliari, University of Cagliari, Cagliari, Italy
\end{abstract}

The present study analyzed the results of the endoscopic approach to T1, T2 and selected T3 supraglottic carcinoma with the aim of reviewing functional and oncologic outcomes after different types of endoscopic supraglottic laryngectomies. This is a retrospective clinical study of 42 consecutive patients (mean age of 61.8 years, 33 males, 9 females) treated by the senior author for supraglottic squamous cell carcinoma with a transoral $\mathrm{CO}_{2}$ laser approach and reviewed from November 2010 to September 2017. Surgical procedures were classified according to the European Laryngological Society. In addition to the standardized transoral supraglottic laryngectomies, we introduced a modified type IVb by sparing the inferior third of the arytenoid if not directly involved in the tumor. Swallowing was evaluated with the Swallowing Performance Status Scale reported by the Multinational Association of Supportive Care in Cancer/International Society of Oral Oncology. Survival probabilities were estimated using Kaplan-Meier curves. Two type I, 2 type Ila, 2 type Ilb, 3 type IIla, 12 type IIlb, 13 type IVa, 3 type modified IVb, and 5 type IVb supraglottic laryngectomies were performed. Twenty-one patients (50\%) underwent primary neck dissection. The pathologic TNM classification according to the 8th edition of the American Joint Committee on Cancer system was as follows: 9 pT1cN0, 2 pT1N0, 1 pT1N1, 7 pT2cN0, 1 rypT2cN0, 9 pT2N0, 4 pT2N1, 2 ypT2N1, 2 pT3cN0, 2 rypT3cN0, 1 pT3N1, and 2 pT3N2b. Mean follow-up was 3.4 years (range of 9 months to 6 years). According to the Kaplan-Meier analysis, 5-year disease-specific survival, local-relapse-free survival, nodal-relapse-free survival, overall laryngeal preservation and overall survival of patients without previous head and neck radiotherapy/open surgery were 100\%, 95.2\%, 87.8\%, 100\%, and 64.6\%, respectively. Patients who underwent type I, Ila, and Illb resections $(n=6)$ started oral feeding the day after surgery, patients who underwent type III-IVb modified resections $(n=31)$ started oral feeding 3-4 days after surgery, and patients who underwent standard type $4 \mathrm{~b}$ resections $(n=5)$ started oral feeding 7 days after surgery. Three months after surgery, patients without a clinical history of previous head and neck radiotherapy/open surgery who underwent type III, IVa, and modified IVb resections showed significantly better swallowing compared to patients who underwent standard type IVb resection: grade 4-6 impairment of swallowing in 8 and $66.7 \%$ of cases, respectively $(p=0.006072$ ); patients with a clinical history of previous head and neck radiotherapy/open surgery 
who underwent type III, IVa, and modified IVb resections showed not statistically significant better swallowing compared to patients who underwent standard type IVb resection: grade 4-6 impairment of swallowing at 3 months in $16.7 \%$ and $50 \%$ of cases, respectively $(p=0.23568)$. Transoral $\mathrm{CO}_{2}$ laser supraglottic laryngectomy is an oncologic sound alternative to traditional open neck surgery and chemo-radiotherapy. Recovery of swallowing is significantly worsened after total resection of the arytenoid. Modified type $\mathrm{IVb}$ procedure leaving intact, when possible, the inferior third of the arytenoid and consequently the glottic competence, improves functional outcome.

Keywords: supraglottic carcinoma, endoscopic surgery, $\mathrm{CO}_{2}$ laser, swallowing, microlaryngoscopy, functional results

\section{INTRODUCTION}

Squamous cell carcinoma (SCC) arising from the vestibule, the false cords, and the epiglottis with or without limited extension to the pyriform sinus and to the arytenoid may be treated through an endoscopic and organ-preservation approach that allows for the complete removal of the lesion without the need for "open surgery".

Currently, endoscopic $\mathrm{CO}_{2}$ laser supraglottic laryngectomy (ESL) targets mainly Tis, T1, and T2 tumors involving the epiglottis, the false vocal cords, and the aryepiglottic folds but also selected cases of T3 and T4 supraglottic lesions (1-3).

The choice of the most adequate treatment shouldn't be based only on the oncologic results of a procedure, but also quality of life issues have to be considered. Vocal function is generally not significantly impaired by supraglottic resection except during the wound healing period soon after the surgical procedure, while partial or complete resection of supraglottic structures can be burdened by post-operative aspiration during swallowing (4). The need for a nasogastric feeding tube, tracheostomy and/or a percutaneous endoscopic gastrostomy (PEG) are the main criteria used to evaluate early and long-term post-operative functional impairment $(5,6)$, but recovery of swallowing could be impaired by different degrees of aspiration, and consequently, feeding intake limitations can be observed even in patients without tracheostomy and/or PEG.

Incomplete airway closure has been mainly associated with arytenoid resection $(1,4)$ (corresponding to type IVb supraglottic laryngectomy according to the European Laryngological Society Classification) (3) and has always been considered with caution while planning the treatment strategy as already observed for type $\mathrm{Vb}$ cordectomy.

The present study analyzed the results of a single surgeon's experience (RP) with transoral $\mathrm{CO}_{2}$ laser standardized supraglottic laryngectomy, with the aim of evaluating the functional results after different types of resections. Oncologic outcomes, the role of neck dissection and of the adjuvant radiotherapy were also evaluated.

\section{METHODS}

This is a retrospective clinical study of 42 consecutive patients (mean age of 61.8 years; range of 43-84 years) treated for supraglottic SCC by ESL with a $\mathrm{CO}_{2}$ laser and reviewed from November 2010 to September 2017. The present study was approved by the Ethics Committee "Commissione del Comitato Etico Indipendente della A.O.U. di Cagliari," under protocol number NP/2018/895.

Eleven patients $(26.2 \%)$ had a clinical history of previous surgical treatment for other head and neck malignancies: 5 oral cavity SCC (surgical treatment was associated in 2 cases with adjuvant radiotherapy), 3 open horizontal laryngectomies (surgical treatment was associated in 1 case with adjuvant radiotherapy), and 3 transoral glottic surgery (1 type Va, and 2 type III cordectomies). Two patients underwent ESL after failure of chemo-radiotherapy, and 2 patients underwent ESL after platinum-based neoadjuvant chemotherapy (3 cycles of cisplatinum associated with 5-fluorouracil).

Pre- and intra-operative work-up included objective local examination by flexible and rigid endoscopy coupled in 2013 with narrow band imaging (NBI), panendoscopy with 0 and $30^{\circ}$ rigid scopes associated with IMAGE $1 \mathrm{~S}$ (Storz, Germany) and enhanced contact endoscopy (ECE) (7). Preoperative imaging included computed tomography (CT) and/or magnetic resonance (MR) with contrast medium of the head neck and CT scan with contrast medium of the chest in order to better assess the lymph node involvement, invasion of the pre-epiglottic and paraglottic spaces, contiguous subsites, and distant metastases.

Procedures were performed under general anesthesia through orotracheal or tracheal intubation with a Mallinckrodt tube Athlone, Ireland (I.D. 5.0-7.0 mm). To obtain complete exposure of the larynx, pharyngo-laryngoscopy was carried out using an adjustable bivalve Storz ${ }^{\circledR}$ (Tuttlingen, Germany) pharyngo-laryngoscope (Weerda type). Surgical procedures were classified according to the European Laryngological Society (3). Patients with limited involvement of the anteromedial aspect of the arytenoid underwent modified type $\mathrm{IVb}$ procedure extended to the superior two-thirds of the arytenoid.

The following microscopes were used during the surgical procedure: Zeiss Universal S2 or Zeiss S21 (Jena, Germany) with $400 \mathrm{~mm}$ focal lens coupled with an Acupulse (Tel Aviv, Israel) $\mathrm{CO}_{2}$ laser with an Acublade (Tel Aviv, Israel) focusing system, with which it was possible to obtain a 150 -micron spot. The super-pulse mode was used at 10 watts. Blood vessels larger than $0.5-1 \mathrm{~mm}$ in diameter were 
coagulated with bipolar forceps or clamped with microclips. With respect to the cleavage plane, the laser was always used in cutting mode and never in vaporization mode in order to obtain precise histological information of the entire specimen.

Frozen sections for the intraoperative evaluation of surgical margins were not routinely used in type I-II ESL. Surgical specimens were sent to a dedicated pathologist after marking one designated edge with black ink in the "en bloc" excisions. In "multibloc" procedures, all surgical margins (superficial and deep) were assessed, and the distance between tumor and margins was measured. A surgical margin with more than $1 \mathrm{~mm}$ of healthy tissue around the tumor was considered as "negative," "close" with $1 \mathrm{~mm}$ or less, and "positive" when the tumor was present at the level of one or more superficial and/or deep resection margins (2).

Patients submitted to neck dissection were treated with synchronous or within 30 days delayed neck procedure unilaterally or bilaterally according to the tumor relationship with the midline and the availability of the definitive histology. A wait-and-see policy for the neck was chosen in selected frail patients and in patients with cT1N0 and small cT2N0 lesions (i.e., T2 with a volume comparable to that of a T1 but encroaching on two adjacent subsites, as in the case of a tumor centered between the supra- and infrahyoid epiglottis). All patients with clinically positive nodes were always treated by therapeutic unilateral or bilateral neck dissection performed simultaneously with the transoral procedure.

Temporary tracheostomy was considered according to the extent of the resection, the simultaneous neck dissection, and comorbidities.

A naso-gastric feeding tube was not considered necessary after type I-II resections.

Based on definitive pathologic findings, tumors were re-staged according to the 8th edition of the American Joint Committee on Cancer (AJCC) system (8).

Patients with positive margins were submitted after counseling to endoscopic enlargement usually at the time of neck dissection (when delayed).

Patients with neoplastic perineural and vascular invasion, multiple positive lymph nodes, and/or extracapsular spread underwent adjuvant radiotherapy/chemo-radiotherapy (9).

After healing the surgical wound, voice and swallowing rehabilitation and regular follow-up were planned according to National Comprehensive Cancer Network (NCCN) guidelines (10).

Swallowing was evaluated on the basis of the Swallowing Performance Status Scale (SPS) reported by the Multinational Association of Supportive Care in the Cancer/International Society of Oral Oncology (11):

- Grade 1: normal

- Grade 2: patients show functional limits but is able to eat a regular diet without modifications or swallowing precautions.

- Grade 3: patients show mild impairment or mild dysfunction that requires a modified diet without need for therapeutic swallowing precautions.
- Grade 4: patients show mild-to-moderate impairment and require a modified diet and therapeutic precautions to minimize aspiration risk.

- Grade 5: patients show moderate impairment or moderate dysfunction with aspiration seen on exam that requires a modified diet and swallowing precautions to minimize aspiration events.

- Grade 6: patients show moderate-to-severe dysfunction with aspiration seen on exam that requires a modified diet and swallowing precautions to minimize aspiration events, or supplemental enteral feeding support.

- Grade 7: patients show severe impairment or severe dysfunction with significant aspiration or inadequate transit to the esophagus that requires primary enteral feeding support.

Objective analysis of the dysphagia included a swallowing test performed under fibrolaryngoscopy, with stained food and fluid, and videofluoroscopy to objectivate subclinical laryngotracheobronchial aspiration.

Patients included in the present study were followed up from the date of surgery until, when possible, February 2018.

Survival probabilities over time were estimated using KaplanMeier curves and Cox proportional hazard models. Statistical analyses were performed using GraphPad Prism software (GraphPad, San Diego, CA, USA). Time-related study endpoints were overall survival (OS), disease-specific survival (DSS), local recurrence free survival, nodal recurrence free survival, and overall laryngeal preservation rate (OLP). Log-rank (MantelCox) tests were applied to compare survival rates between the different patient groups ( $\mathrm{pT}$ category, patients with or without previous head and neck radiotherapy/open surgery, and cN0/cN1 patients who underwent primary neck dissection vs. patients who underwent close follow-up). The Pearson $X^{2}$ test was applied to compare categorical variables. A $p$-value $<0.05$ was considered significant.

Functional results were evaluated differentiating two groups of patients: those with a clinical history of previous head and neck radiotherapy/open surgery that could have impaired swallowing before endoscopic management $(n=10)$ vs. naive patients $(n=32)$; patients never treated before with head and neck radiotherapy/open surgery (naive). Patients previously treated endoscopically for glottic tumors $(n=3)$ and those who underwent neoadjuvant therapy $(n=2)$ were considered as naive patients because the endoscopic management of glottic tumors has minimal effects on swallowing (none of the patients included in the present study was treated by a cordectomy extended to the arytenoid or other supraglottic areas), and because neoadjuvant therapy was part of the conservative approach.

The literature review was based on an extensive MEDLINE search using "supraglottic cancer," "transoral," "endoscopy," "CO $\mathrm{CO}_{2}$ laser," "microsurgery," and "functional results" as keywords.

\section{RESULTS}

This study included 33 males (mean age of 63.9 years; range of 44-84 years) and 9 females (mean age of 55 years; range of 43-74 years). 
Daily alcohol consumption was reported by 30 patients (71.4\%), and daily tobacco consumption was reported by 32 patients $(76.2 \%)$.

The following resections were performed: 2 type I, 2 type IIa, 2 type IIb, 3 type IIIa, 12 type IIIb, 13 type IVa, 3 modified type IVb, and 5 type IVb supraglottic laryngectomies. Twenty-one patients (50\%) staged as cT1N0 in 2 cases, cT2N0 in 2 cases, cT3N0 in one case, cT1N1 in one case, cT2N1 in 13 cases, cT3N1 in one case, and cT3N2b in one case underwent primary neck dissection (16 simultaneously and 5 within 30 days after the transoral procedure), 7 patients (16.7\%) with a clinical history of neck dissection for previous head and neck malignancies (bilateral in 5 cases and unilateral in 2 cases) did not undergo neck dissection, and 14 patients $(33.3 \%)$ underwent a close wait-and-see policy of the neck status.

In 23 cases (54.8\%), temporary tracheostomy was performed.

One patient experienced post-operative bleeding that was controlled under general anesthesia.

Patients who underwent type I, IIa and IIb resections $(n=6)$ started oral feeding the day after surgery, while a naso-gastric feeding tube was considered necessary in the other 36 patients $(85.7 \%$ of the series, mean time of persistence of the naso-gastric feeding tube of 5.9 days, range of 318 days). Patients who underwent type IIIa, IIIb, IVa, and modified IVb resections $(n=31)$ started oral feeding 34 days after surgery, and patients who underwent standard type $\mathrm{IVb}$ resections $(n=5)$ started oral feeding 7 days after surgery. The need for a nasogastric feeding tube was longer in patients who underwent standard type IVb resections (19 days vs. 9 days in patients who underwent type IVa and modified $\mathrm{IVb}$ resections). No patient had percutaneous endoscopic gastrostomy (PEG). The time of hospitalization, permanence of tracheostomy and of naso-gastric feeding tube are detailed in Table 1.

Thirty-four procedures (81\%) were performed in free margins of resection. Four procedures (9.5\%) showed close margins: one patient underwent surgical enlargement (definitive histology of the enlargement did not find residual tumor), and 3 were submitted to a wait-and-see policy; among them, one patient experienced recurrence on primary and underwent total laryngectomy. One procedure (2.4\%) was performed with margins involved by mild dysplasia, and the patient underwent surgical enlargement (histology showed residual mild dysplasia removed with free margins). In 3 cases (7.1\%), the excision was performed with positive deep margins: two patients underwent surgical enlargement (no evidence of residual disease after definitive histology), and one patient underwent radiotherapy.

TABLE 1 | Mean hospitalization time, need for temporary tracheostomy, and need for naso-gastric feeding tube for each type of transoral supraglottic laryngectomy/group of patients.

\begin{tabular}{|c|c|c|c|}
\hline & $\begin{array}{c}\text { Mean hospitalization } \\
\text { time (days) }\end{array}$ & $\begin{array}{c}\text { Permanence of temporary } \\
\text { tracheostomy (days)/Number of } \\
\text { patients who underwent tracheostomy }\end{array}$ & $\begin{array}{c}\text { Permanence of naso-gastric } \\
\text { feeding tube (days) }\end{array}$ \\
\hline All patients $(n=42)$ & 9.8 & $9.7 / 23$ & 9.6 \\
\hline Type I ESL $(n=2)$ & 4 & $3 / 1$ & - \\
\hline Type II ESL $(n=4)$ & 6 & $3 / 1$ & - \\
\hline Type III ESL $(n=15)$ & 10 & $9 / 9$ & 7 \\
\hline Type IVa ESL $(n=13)$ & 10 & $10 / 8$ & 9 \\
\hline Type IVb mod ESL $(n=3)$ & 10 & $10 / 1$ & 9 \\
\hline Type IVb ESL $(n=5)$ & 14 & $15 / 3$ & 19 \\
\hline Naive patients $(n=32)$ & 9.6 & $9.6 / 19$ & 7.7 \\
\hline Type I ESL $(n=1)$ & 3 & - & - \\
\hline Type II ESL $(n=3)$ & 6.5 & $3 / 1$ & - \\
\hline Type III ESL $(n=14)$ & 10 & $9 / 9$ & 7 \\
\hline Type IVa ESL $(n=9)$ & 10 & $10 / 6$ & 8.6 \\
\hline Type IVb mod ESL $(n=2)$ & 10.5 & $10 / 1$ & 9 \\
\hline Type IVb ESL $(n=3)$ & 11.3 & $14 / 2$ & 17.5 \\
\hline Patients with previous treatments $(n=10)^{\star}$ & 10.4 & $10 / 4$ & 10 \\
\hline Type I ESL $(n=1)$ & 5 & $3 / 1$ & - \\
\hline Type II ESL $(n=1)$ & 4.5 & - & - \\
\hline Type III ESL $(n=1)$ & 9 & - & 7 \\
\hline Type IVa ESL $(n=4)$ & 10 & $10 / 2$ & 10 \\
\hline Type IVb mod ESL $(n=1)$ & 9 & - & 9 \\
\hline Type IVb ESL $(n=2)$ & 18 & $17 / 1$ & 22 \\
\hline
\end{tabular}

*Patients with a clinical history of previous head and neck radiotherapy/open surgery. 
The pathologic TNM classification according to the AJCC system (8) was as follows: 9 pT1cN0, 2 pT1N0, one pT1N1, 7 pT2cN0, one rypT2cN0, 9 pT2N0, 4 pT2N1, 2 ypT2N1, 2 pT3cN0, 2 rypT3cN0, one pT3N1, and 2 pT3N2b.

Eight patients (19\%) underwent adjuvant radiotherapy (Table 2).

Three months after surgery, naive patients who underwent type III, IVa, and modified IVb resections showed significantly better swallowing compared to patients who underwent standard type IVb resection: grade 4-6 impairment of swallowing in 8 and $66.7 \%$ of cases, respectively $(p=0.006072)$, as shown in Table 3; one patient was submitted to a formal type IVb ESL and experienced two episodes of aspiration pneumonia but refused the proposed PEG or total laryngectomy. Patients of the second group who underwent type III, IVa, and modified IVb resections showed not statistically significant better swallowing compared to patients who underwent standard type IVb resection: grade 46 impairment of swallowing at 3 months in 16.7 and 50\% of cases, respectively ( $p=0.23568$ ), as shown in Table 4 .

The mean follow-up was 3.4 years (range of 9 months to 6 years).

During the 5-year follow-up, 11 patients died from other causes (among them, 2 patients died from other causes within one year after surgery): pulmonary carcinoma in 5 cases, liver cancer in one case, hearth failure in one case, stroke in 2 cases, pulmonary embolism in one case, and unknown in one case), and 2 patients died from the disease.

Nine patients $(21.4 \%)$ experienced recurrence of the disease as shown in Table 5 ( 4 on ipsilateral neck, one on contralateral neck, one on ipsilateral neck and after one year on primary and three on primary) and underwent total laryngectomy $(n=3)$, neck dissection $(n=6)$, and open horizontal laryngectomy $(n=1)$. Three patients underwent adjuvant radiotherapy after rescue therapy. Kaplan-Meier analysis revealed 5-year disease-specific survival, local-relapse-free survival, nodal-relapse-free survival, overall laryngeal preservation and overall survival of 93.1, 90.5, 83, 90.7, and 64.9\%, respectively (Table 6).

The Cox univariate analysis showed that patients with a clinical history of previous head and neck radiotherapy/open surgery $(n=10)$ experienced statistically significant worse local recurrence free survival, and OLP compared to naive patients $(n=32): 65.6$ vs. $95.2 \%$ ( $p=0.0181$; hazard ratio of 9$)$, and 65.6 vs. $100 \%$ ( $p=0.0025$; hazard ratio of 9 ) respectively, while they experienced worse but not statistically significant nodal recurrence free survival: 70 vs. $87.8 \%(p=0.1603$; hazard ratio of 7.7).

The Cox univariate analysis showed that $\mathrm{cN} 0$ patients who did not undergo primary neck dissection $(n=21)$ experienced lower but not statistically significant nodal recurrence free survival compared to $\mathrm{cN} 0$ and $\mathrm{cN} 1$ patients who underwent primary neck

\begin{tabular}{|c|c|c|c|c|c|c|c|c|c|c|}
\hline $\begin{array}{l}\text { N/Sex/ } \\
\text { Age } \\
\text { (years) }\end{array}$ & cTNM & $\begin{array}{l}\text { Type } \\
\text { of ESL }\end{array}$ & pTNM & Margins & $\begin{array}{l}\text { Surgical } \\
\text { enlargement }\end{array}$ & $\begin{array}{l}\text { Vascular } \\
\text { invasion }\end{array}$ & Nodal involvement & $\begin{array}{l}\text { Dosage of } \\
\text { radiotherapy }\end{array}$ & $\begin{array}{l}\text { Associated } \\
\text { chemotherapy }\end{array}$ & Outcomes \\
\hline 1/M/67 & cT2 N1 & $\mathrm{IVa}$ & pT2 N1 & $\begin{array}{l}\text { Involved } \\
\text { (deep margin) }\end{array}$ & $\begin{array}{l}\text { Yes (No residual } \\
\text { tumor) }\end{array}$ & Yes & $\begin{array}{l}1 \text { ipsilateral lymph } \\
\text { node (extracapsular } \\
\text { spread) }\end{array}$ & 66 Gy & No & $\begin{array}{l}\text { NED after } 1 \\
\text { year of } \\
\text { follow-up }\end{array}$ \\
\hline 2/F/70 & cT2 NO & IVa & pT2 cNO & $\begin{array}{l}\text { Involved } \\
\text { (deep margin) }\end{array}$ & No & No & No & 66 Gy & $\begin{array}{l}8 \text { cycles of } \\
\text { CISPLATIN }\end{array}$ & $\begin{array}{l}\text { NED after } 5.3 \\
\text { years of } \\
\text { follow-up }\end{array}$ \\
\hline 3/M/63 & cT1 N1 & Illb & pT2 N1 & Free & No & No & $\begin{array}{l}1 \text { ipsilateral lymph } \\
\text { node }\end{array}$ & 54 Gy & $\begin{array}{l}12 \text { cycles of } \\
\text { CISPLATIN }\end{array}$ & $\begin{array}{l}\text { DOC after } 4.2 \\
\text { years of } \\
\text { follow-up }\end{array}$ \\
\hline 4/F/52 & cT2 N2b & $\mathrm{IVa}$ & pT3 N2b & $\begin{array}{l}\text { Involved } \\
\text { (deep margin) }\end{array}$ & $\begin{array}{l}\text { Yes (No residual } \\
\text { tumor) }\end{array}$ & No & $\begin{array}{l}\text { Multiple (2) ipsilateral } \\
\text { lymph nodes }\end{array}$ & 66 Gy & No & $\begin{array}{l}\text { NED after } 5.8 \\
\text { years of } \\
\text { follow-up }\end{array}$ \\
\hline 5/M/63 & $\begin{array}{l}\text { cyT2 } \\
\mathrm{N} 2 \mathrm{c}^{\star \star}\end{array}$ & Illb & урт2 N1 & Free & No & No & $\begin{array}{l}1 \text { ipsilateral lymph } \\
\text { node }\end{array}$ & 54 Gy & No & $\begin{array}{l}\text { NED after } 5 \\
\text { years of } \\
\text { follow-up }\end{array}$ \\
\hline 6/F/57 & cT2 $\mathrm{NO}^{\star}$ & IVa & pT3 cNO & Free & No & No & No & 54 Gy & No & $\begin{array}{l}\text { NED after } 3.5 \\
\text { years of } \\
\text { follow-up }\end{array}$ \\
\hline 7/M/61 & cT2 N2a & $\begin{array}{l}\text { Modified } \\
\text { IVb }\end{array}$ & pT3 N2b & $\begin{array}{l}\text { Close (deep } \\
\text { margin) }\end{array}$ & Yes (No residual & No & $\begin{array}{l}1 \text { ipsilateral lymph } \\
\text { node (extracapsular } \\
\text { spread) }\end{array}$ & 66 Gy & No & $\begin{array}{l}\text { DOC after } 1.6 \\
\text { years of }\end{array}$ \\
\hline 8/M/80 & cT1 No & $\mathrm{IVa}$ & PT2 N1 & Free & No & No & $\begin{array}{l}1 \text { ipsilateral lymph } \\
\text { node }\end{array}$ & 54 Gy & No & $\begin{array}{l}\text { DOC after } 0.7 \\
\text { years of } \\
\text { follow-up }\end{array}$ \\
\hline
\end{tabular}

*Patient with a clinical history of previous head and neck open surgery.

${ }^{\star *}$ Patient who previously underwent neoadjuvant chemotherapy. 
TABLE 3 | Swallowing results according to the Swallowing Performance Status Scale of all patients without history of previous head and neck radiotherapy/open surgery.

\begin{tabular}{|c|c|c|c|c|c|c|c|}
\hline $\begin{array}{l}\text { Degrees of } \\
\text { dysphagia }\end{array}$ & All series & $\begin{array}{l}\text { Type I } \\
\text { resection }\end{array}$ & $\begin{array}{l}\text { Type II } \\
\text { resection }\end{array}$ & $\begin{array}{l}\text { Type III } \\
\text { resection }\end{array}$ & $\begin{array}{l}\text { Type IVa } \\
\text { resection }\end{array}$ & $\begin{array}{c}\text { Type IVb } \\
\text { modified resection }\end{array}$ & $\begin{array}{l}\text { Type IVb } \\
\text { resection }\end{array}$ \\
\hline Grade 1 & 15 & 1 & 3 & 8 & 3 & & \\
\hline Grade 3 & 5 & & & $1(1)^{\star}$ & $2^{*}$ & $1^{*}$ & 1 \\
\hline Grade 4 & 2 & & & $1^{*}$ & $1^{*}$ & & \\
\hline \multicolumn{8}{|l|}{ Grade 7} \\
\hline Total & $32(7)^{\star}$ & 1 & 3 & $14(2)^{\star}$ & $9(4)^{\star}$ & $2(1)^{\star}$ & 3 \\
\hline
\end{tabular}

${ }^{\star}$ Number of patients who underwent adjuvant radiotherapy.

TABLE 4 | Swallowing results according to the Swallowing Performance Status Scale of all patients who previously underwent head and neck radiotherapy/open surgery.

\begin{tabular}{|c|c|c|c|c|c|c|c|}
\hline $\begin{array}{l}\text { Degrees of } \\
\text { dysphagia }\end{array}$ & All series & $\begin{array}{l}\text { Type I } \\
\text { resection }\end{array}$ & $\begin{array}{l}\text { Type II } \\
\text { resection }\end{array}$ & $\begin{array}{l}\text { Type III } \\
\text { resection }\end{array}$ & $\begin{array}{l}\text { Type IVa } \\
\text { resection }\end{array}$ & $\begin{array}{c}\text { Type IVb } \\
\text { modified resection }\end{array}$ & Type IVb resection \\
\hline Grade 1 & 2 & 1 & & & 1 & & \\
\hline Grade 3 & 3 & & & 1 & & 1 & 1 \\
\hline Grade 4 & 2 & & & & 1 & & 1 \\
\hline \multicolumn{8}{|l|}{ Grade 7} \\
\hline Total & $10(1)^{\star}$ & 1 & 1 & 1 & $4(1)^{\star}$ & 1 & 2 \\
\hline
\end{tabular}

${ }^{*}$ Number of patients who underwent adjuvant radiotherapy.

dissection $(n=21)$ : $74.7 \%$ vs. $92.9 \%$ ( $p=0.1013$; hazard ratio of 4.6$)$.

\section{DISCUSSION}

Alonso first described the supraglottic laryngectomy as a conservative treatment for supraglottic tumors through an external approach (12), but the first author who described transoral $\mathrm{CO}_{2}$ laser surgery for the removal of a supraglottic carcinoma was Vaughan in 1978 (13). The endoscopic approach has been progressively reported as an alternative to open neck supraglottic surgery (14), allowing for a radical resection, tailored on the basis of the real extension of the tumor.

Pre- and intra-operative staging is based on the precise endoscopic evaluation of the superficial extension of the neoplasms that can be performed with image-enhanced endoscopy in the pre-operative setting, and ECE used to possibly detect synchronous satellite lesions and to spot very early pre-cancerous lesions that can progress if left untreated. As reported in literature, inadequate laryngeal exposure, arytenoid fixation, massive invasion of the pre-epiglottic space, and laryngeal framework involvement still represent the "weak points" of the endoscopic approach, reducing its application in terms of technical feasibility and local control compared to more traditional surgical and non-surgical therapies (2). Vocal cord mobility must be carefully evaluated: in supraglottic cancer the tumor can easily extent to the arytenoid with encroachment of the cricoarytenoid joint. Patients with supraglottic lesions usually undergo CT or MRI with contrast medium also to spot the preepiglottic space and to check for neck nodes and/or distant metastases. In our series, pre-operative evaluation failed to detect early T3 lesions as three patients previously staged as cT2 were up-staged to pT3 after definitive histology for paraglottic involvement not evident at imaging. Cancer of the "3-folds" could spread in different directions, superficially toward base of tongue, pyriform sinus, false vocal cords and epiglottis but deep invasion can involve the content of the superior paraglottic space; in this condition imaging plays an important role for the detection of the deep invasion (15), but in some instances only histology confirms its involvement, upstaging the class of tumor; this is also the most frequently area in which vascular invasion or perineural spread is detected at histology giving the indication for adjuvant therapy (9) as in our series.

Platinum-based induction chemotherapy regimens in patients with intermediate/advanced laryngeal carcinoma have been reported in literature to down-stage T3 trans glottic carcinoma by Laccourreye et al. allowing for remobilization of the fixed 


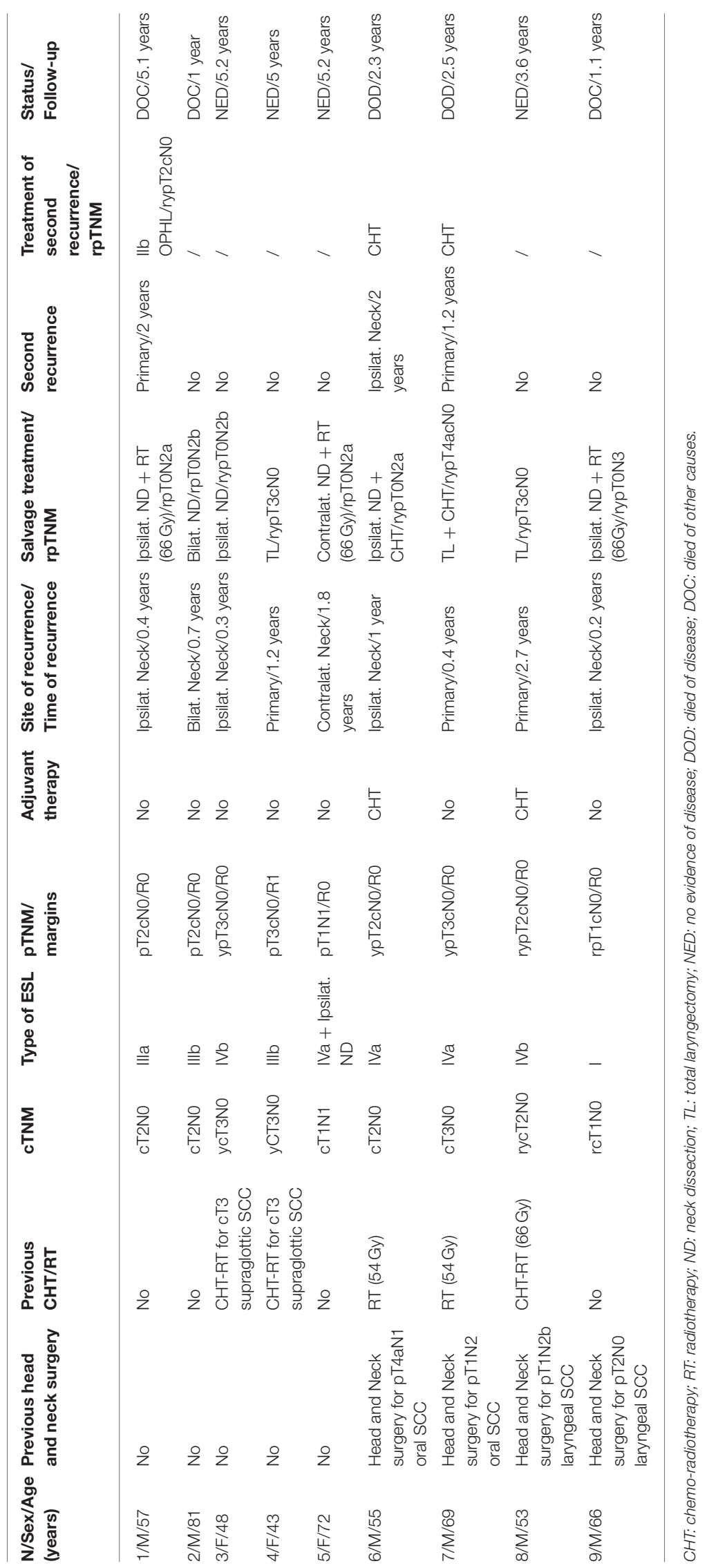


TABLE 6 | 5-year survival outcomes.

\begin{tabular}{|c|c|c|c|c|c|}
\hline & $\begin{array}{l}\text { Disease- } \\
\text { specific } \\
\text { survival }\end{array}$ & $\begin{array}{l}\text { Local } \\
\text { recurrence-free } \\
\text { survival }\end{array}$ & $\begin{array}{c}\text { Nodal } \\
\text { recurrence-free } \\
\text { survival }\end{array}$ & $\begin{array}{l}\text { Overall laryngeal } \\
\text { preservation }\end{array}$ & Overall survival \\
\hline All series $(n=42)$ & $\begin{array}{l}93.1 \% \\
\text { SE: } 4.7\end{array}$ & $\begin{array}{l}90.5 \% \\
\text { SE: } 6 \%\end{array}$ & $\begin{array}{c}83 \% \\
\text { SE: } 6.3 \%\end{array}$ & $\begin{array}{c}90.7 \% \\
\text { SE: } 5.2 \%\end{array}$ & $\begin{array}{c}64.9 \% \\
\text { SE: } 8.8 \%\end{array}$ \\
\hline $\mathrm{T} 1(n=12)$ & $100 \%$ & $100 \%$ & $\begin{array}{l}80.1 \% \\
\text { SE: } 6 \%\end{array}$ & $100 \%$ & $\begin{array}{l}70 \% \\
\text { SE: } 6 \%\end{array}$ \\
\hline $\mathrm{T} 2(n=23)$ & $\begin{array}{l}95.7 \% \\
\text { SE: } 4 \%\end{array}$ & $\begin{array}{l}91.3 \% \\
\text { SE: } 5 \%\end{array}$ & $\begin{array}{l}83.6 \% \\
\text { SE: } 7 \%\end{array}$ & $\begin{array}{l}95.7 \% \\
\text { SE: } 4 \%\end{array}$ & $\begin{array}{l}66 \% \\
\text { SE: } 6 \%\end{array}$ \\
\hline T3 $(n=7)$ & $\begin{array}{l}71.4 \% \\
\text { SE: } 14 \%\end{array}$ & $\begin{array}{l}71.4 \% \\
\text { SE: } 14 \%\end{array}$ & $\begin{array}{l}85.7 \% \\
\text { SE: } 13 \%\end{array}$ & $\begin{array}{l}71.4 \% \\
\text { SE: } 14 \%\end{array}$ & $\begin{array}{c}43 \% \\
\text { SE: } 14 \%\end{array}$ \\
\hline Naive patients $(n=32)$ & $100 \%$ & $\begin{array}{l}95.2 \% \\
\text { SE: } 4.6 \%\end{array}$ & $\begin{array}{l}87.8 \% \\
\text { SE: } 6.3 \%\end{array}$ & $100 \%$ & $\begin{array}{l}64.6 \% \\
\text { SE:16.3\% }\end{array}$ \\
\hline $\mathrm{T} 1(n=9)$ & $100 \%$ & $100 \%$ & $\begin{array}{c}85.7 \% \\
\text { SE: } 13.2 \%\end{array}$ & $100 \%$ & $\begin{array}{c}75 \% \\
\text { SE: } 18.9 \%\end{array}$ \\
\hline $\mathrm{T} 2(n=20)$ & $100 \%$ & $\begin{array}{l}91.7 \% \\
\text { SE: } 8 \%\end{array}$ & $\begin{array}{l}87.8 \% \\
\text { SE: } 8 \%\end{array}$ & $100 \%$ & $\begin{array}{c}65 \% \\
\text { SE: } 12.8 \%\end{array}$ \\
\hline T3 $(n=3)$ & $100 \%$ & $100 \%$ & $100 \%$ & $100 \%$ & $\begin{array}{c}66.7 \% \\
\text { SE: } 27.2 \%\end{array}$ \\
\hline Previously treated $(n=10)$ & $\begin{array}{c}77.8 \% \\
\text { SE: } 13.9 \%\end{array}$ & $\begin{array}{c}65.6 \% \\
\text { SE: } 16.4 \%\end{array}$ & $\begin{array}{c}70 \% \\
\text { SE: } 14.5 \%\end{array}$ & $\begin{array}{c}65.6 \% \\
\text { SE: } 16.4 \%\end{array}$ & $\begin{array}{c}70 \% \\
\text { SE: } 14.5 \%\end{array}$ \\
\hline T1 (3) & $100 \%$ & $100 \%$ & $\begin{array}{c}66.7 \% \\
\text { SE: } 27.2 \%\end{array}$ & $100 \%$ & $\begin{array}{c}66.7 \% \\
\text { SE: } 27.2 \%\end{array}$ \\
\hline T2 (3) & $\begin{array}{c}66.7 \% \\
\text { SE: } 27.2 \%\end{array}$ & $\begin{array}{c}50 \% \\
\text { SE: } 35.6 \%\end{array}$ & $\begin{array}{c}66.7 \% \\
\text { SE: } 27.2 \%\end{array}$ & $\begin{array}{c}50 \% \\
\text { SE: } 35.4 \%\end{array}$ & $\begin{array}{c}66.7 \% \\
\text { SE: } 27.2 \%\end{array}$ \\
\hline T3 (4) & $\begin{array}{c}75 \% \\
\text { SE: } 21.7 \%\end{array}$ & $\begin{array}{c}50 \% \\
\text { SE: } 25 \%\end{array}$ & $\begin{array}{c}75 \% \\
\text { SE: } 21.7 \%\end{array}$ & $\begin{array}{c}50 \% \\
\text { SE: } 25 \%\end{array}$ & $\begin{array}{c}75 \% \\
\text { SE: } 21 \%\end{array}$ \\
\hline
\end{tabular}

SE: standard error.

arytenoid cartilage who became amenable to conservative surgical approach (16). In our series platinum-based induction chemotherapy was performed in two patients with bulky T2 and although TNM staging did not change, we think that the transoral approach was facilitated by the reduction of the volume of the tumor in at least one patient. On the basis of this limited experience we are not able to state that neoadjuvant chemotherapy followed by ESL has a real role on organpreservation strategies and more studies are needed.

Every effort should be made to obtain clear margins during the first endoscopic procedure or with a second-look surgery to reduce any over-treatment or multimodal therapies (14). Piecemeal resection allows for the better management of bulky lesions, apparently without consequences for dissemination of neoplastic cells to the neck or distant subsites (17).

In case of close margins or involved margins, the following policy was followed: a radicalization was performed within 4 weeks after surgery in 1 over 4 patients with close margins (the remaining underwent a wait-and-see policy), while 3 over 4 cases with positive margins underwent a second look with enlargement of the surgical margins (in 1 case it was coupled with the delayed neck dissection), and one patient underwent adjuvant radiotherapy (positive deep margin). Seven patients who showed close or positive margins did not experience recurrent carcinoma, while one patient with close margin (clinical history of previous chemo-radiotherapy before the ESL) addressed for a wait-and-see policy, experienced a relapse on primary after 1.2 years of followup and underwent total laryngectomy as a salvage procedure.

Tumors of the 3-fold area extending to neighboring subsites can be treated by extended ESL, such as type IVa resection, including the free edge of the epiglottis, the 3-fold area and the ventricular fold. In such cases, the resection may include the inner or medial wall and anterior corner of the pyriform sinus without major functional consequences. In case of extension of the neoplasm to the anterior/superior aspect of a mobile arytenoid, the arytenoid can be included in the resection (type $\mathrm{IVb}$ ), but swallowing can be greatly impaired (1), while an endoscopic approach for patients with lesions extending to the cricoarytenoid joint should be avoided. We performed a modified type $\mathrm{IVb}$ resection in 3 patients, leaving intact the inferior third of the arytenoid including the vocal process, thereby maintaining glottic competence. Oncologic outcomes in these cases were not affected by such a tailored resection.

During types IIIb and IV ESL, the superior laryngeal artery was constantly ligated with metallic clips in order to reduce the risk of post-operative massive hemorrhage that has been reported 
TABLE 7 | Comparative results of functional results from different studies.

\begin{tabular}{|c|c|c|c|c|}
\hline $\begin{array}{l}\text { Author and year of } \\
\text { publication }\end{array}$ & $\begin{array}{l}\text { No. of procedures/T } \\
\text { stages }\end{array}$ & $\begin{array}{c}\text { No. of early } \\
\text { post-operative } \\
\text { complications (\%) }\end{array}$ & $\begin{array}{l}\text { No. of permanent } \\
\text { tracheostomies (\%) }\end{array}$ & $\begin{array}{l}\text { No. of patients who } \\
\text { required additional } \\
\text { procedures to avoid } \\
\text { aspiration (\%) }\end{array}$ \\
\hline Puxeddu et al. (14) & $\begin{array}{l}12 \\
\mathrm{~T} 1: 3 \\
\mathrm{~T} 2: 9\end{array}$ & $\begin{array}{c}1 \\
(8.3 \%)\end{array}$ & $\begin{array}{c}0 \\
(0 \%)\end{array}$ & $\begin{array}{c}0 \\
(0 \%)\end{array}$ \\
\hline $\begin{array}{l}\text { Bernal-Sprekelsen } \\
\text { et al. (5) }\end{array}$ & $\begin{array}{l}121 \\
\mathrm{~T} 1: 16 \\
\mathrm{~T} 2: 41 \\
\mathrm{~T} 3: 53 \\
\mathrm{~T} 4: 11\end{array}$ & I & $\begin{array}{c}2 \\
(1.7 \%)\end{array}$ & $\begin{array}{c}2 \\
(1.7 \%)\end{array}$ \\
\hline Piazza et al. (1) & $\begin{array}{l}96 \\
\mathrm{~T} 1: 28 \\
\mathrm{~T} 2: 46 \\
\mathrm{~T} 3: 22\end{array}$ & $\begin{array}{c}10 \\
(11 \%)\end{array}$ & $\begin{array}{c}0 \\
(0 \%)\end{array}$ & $\begin{array}{c}0 \\
(0 \%)\end{array}$ \\
\hline Bertolin et al. (26) & $\begin{array}{l}15^{\star} \\
\mathrm{T} 1: 2 \\
\mathrm{~T} 2: 12 \\
\mathrm{~T} 3: 1\end{array}$ & / & $\begin{array}{c}0 \\
(0 \%)\end{array}$ & $\begin{array}{c}0 \\
(0 \%)\end{array}$ \\
\hline
\end{tabular}

*Patients included in the study with the analysis of the functional results.

as responsible for major complications (17). Minor bleeding in the present series occurred in one patient and required general anesthesia and bipolar cautery.

Kaplan-Meier analysis of the entire cohort of patients showed a 5-year local relapse-free survival of $90.5 \%$ and overall laryngeal preservation rate of $90.7 \%$, showing that the endoscopic approach tailored to supraglottic carcinoma is an oncologic sound approach for the primary tumor. Local control after ESL and rescue therapy for each class of tumor is described in Table 6.

Since the incidence of occult metastases in patients with supraglottic carcinoma ranges from 20 to $40 \%$ of clinically negative necks (18-20), rising also for moderate to advanced carcinomas, neck dissection should be strongly considered for cN0 patients with supraglottic carcinoma (18). In our series, 8 out of the $26 \mathrm{cN} 0$ patients (30.8\%) showed occult metastases at histology or during follow-up, and patients who did not undergo primary neck dissection experienced lower but not statistically significant ( $p=0.1252$ ) nodal-relapse-free survival (74.7\%) compared to $\mathrm{cN} 0$ and $\mathrm{cN} 1$ patients who underwent primary neck dissection (92.9\%) despite the $\mathrm{T}$ stage. In our series, naive $\mathrm{T} 1$ showed neck metastasis in 1 case $(11.1 \%)$, naive $\mathrm{T} 2$ in 8 cases (40\%), and naive T3 in 2 cases (66.7\%). As a consequence, as from the present series, neck dissection should be planned in almost all patients with supraglottic SCC, in agreement with UK guidelines (9), and a wait-and-see policy should be limited to very early primaries (Tis, small T1 of the superficial border of the epiglottis and of the ary-epiglottic fold) or in case of severe comorbidities. Sentinel node biopsy (SLNB) has been proposed for laryngeal cancer and has a very high negative predictive value (NPV), but the significance of the high NPV of SLNB outside the oral cavity is limited, and further studies are needed, especially for supraglottic carcinoma $(21,22)$.

After surgery, the choice among complementary radiotherapy or chemo-radiotherapy should be balanced in face of margin status, metastatic lymph nodes, pathologic risk factors and patient characteristics (9). In the present series, adjuvant radiotherapy was considered necessary in six patients with metastatic nodes, in one patient treated for a second advanced head and neck malignancy, and in one patient after endoscopic removal of bulky lesions with vascular invasion and deep margin involvement seen at histology.

As shown in Table 6, patients who previously underwent head and neck radiotherapy/open surgery (other head and neck malignancies in 8 cases and failure of chemo-radiotherapy in 2 cases) experienced a statistically significant worse local recurrence free survival and OLP rates compared to naive patients. Consequently, previous treatment for other head and 
neck cancer, including radiotherapy extending to the supraglottic area and recurrent supraglottic cancer, should be carefully considered before ESL; in such cases, small lesions in the supraglottis as usually spotted during follow-up for different head and neck primary tumors can be considered still good candidates for ESL.

Salvage surgery after failed $\mathrm{CO}_{2}$ laser transoral microsurgery in supraglottic tumors may be performed by ESL or, if necessary by all the different open partial horizontal laryngectomy (OPHL) and total laryngectomy. In our series, 4 patients (one naive patient and 3 previously treated with radiotherapy/open surgery) developed a recurrence on primary after ESL. The naive patient underwent a type IIb OPHL, while the 3 patients previously treated with radiotherapy/open surgery underwent total laryngectomy.

Five-year OLP was $100 \%$ in the group of naive patients and $65.6 \%$ in the second group of patients.

Second malignancies have been often observed in patients treated for supraglottic carcinoma (2), and in our series, 5 patients died for a second pulmonary malignancy, supporting the importance of regular chest CT during follow-up.

Temporary tracheostomy in patients undergoing endoscopic laser surgery for supraglottic lesions should be taken carefully into consideration when planning the extent of the resection in order to prevent aspiration and possible passage of massive amounts of blood in the case of post-operative hemorrhage (14). Furthermore, disorders with swallowing and breathing problems can be easily overcome by means of temporary tracheostomy. In our series temporary tracheostomy showed paramount benefit in one patient who experienced post-operative bleeding, and all patients submitted to temporary tracheostomy did not experience long-term complications.

Functional results after $\mathrm{CO}_{2}$ laser supraglottic laryngectomy have been considered in the literature, but few studies differentiated the long-term outcomes on the basis of the different endoscopic resections (1). ESL generally allows for shorter time for swallowing recovery, reduced tracheostomy maintenance, and lower incidence of aspiration pneumonia (18) because of the complete preservation of the sensation of the vestibule and hypopharynx by sparing the superior laryngeal nerve in the majority of the cases, of the accessory muscles that normally contribute to the craniocaudal movements of the larynx, and of an effective cough reflex that may prevent severe problems $(4,23,24)$. However, major dysfunction after ESL, such as permanent tracheostomy and/or PEG, have been reported in the literature (Table 7$)(1,5,6,14,25,26)$. In the present series, we did not perform any PEG, and all tracheostomies were removed within 1 month from surgery. Removal of supraglottic structures can result in various degrees of swallowing problems, even in patients without tracheostomy and/or PEG. The MD Anderson Dysphagia Inventory (MDADI), a subjective dysphagia-specific quality of life scale, has been reported in the literature as a possible instrument $(1,4)$. In our series, the SPS reported by the Multinational Association of Supportive Care in Cancer/International Society of Oral Oncology (11) was preferred since it offers an accurate and objective assessment of the presence and severity of dysphagia and aspiration risk by combining subjective and objective data.

The resection of the epiglottis alone, even when it is completely removed, does not significantly hamper the laryngeal sphincter, which is partially preserved by the adduction of the vestibular bands and the vocal folds (1). In our experience, the resection of the epiglottis was not associated with negative functional outcomes (see Tables 3, 4) such as the resection of the pre-epiglottic content as indeed reported by Peretti et al. $(2,17)$.

Lateral resection of the aryepiglottic fold, mostly when extended to the arytenoid, can hinder the laryngeal competence and, more notably, facilitate the aspiration of the bolus from the pyriform sinus to the laryngeal pathway due to the inadequacy of the barrier between the two. The reduction of the sensitiveness of the upper portion of the pyriform sinus due to resection of the pharyngo-epiglottic fold and its sensitive receptors, and the section of the superior laryngeal nerve may further worse swallowing (1). In our patients, sensation of the vestibule evaluated under fibrolaryngoscopy was generally preserved as the result of the limited traumatism by the $\mathrm{CO}_{2}$ laser since the resection was always performed by tractioning the tissues with minimal power density, reducing the potential injury to the superior laryngeal nerve. The modification of type IVb resection is feasible if there is no major encroachment of the arytenoid or the vocal cord. Although limited to few cases, in our experience, patients who underwent resection extended only to the superior $2 / 3$ of the arytenoid showed significantly better long-term swallowing function than patients who underwent standard type IVb resection $(p=0.029501)$ and had reduced time with naso-gastric feeding tube ( 9 days in patients who underwent IVb modified resections vs. 19 days in patients who underwent standard IVb procedures), highlighting that the residual arytenoid/vocal cord adduction preserves adequate glottic competence. One patient submitted to a formal type IVb ESL experienced two episodes of aspiration pneumonia but refused a proposed PEG or total laryngectomy, and the SPS staging was considered grade 6.

Although adjuvant radiotherapy could be associated with worse functional outcomes (4), our patients who underwent adjuvant radiotherapy experienced minor and not statistically significant worsening of swallowing function $(p=0.1$ 44061).

In conclusion, ESL is an oncologic sound alternative to traditional open neck surgery and radiotherapy and chemoradiotherapy, and, although it requires a higher level of experience in endoscopic surgical technique, it allows to perceive a competitive organ-preservation approach with predictable functional outcomes and acceptable complication rates.

The present study, in agreement with previous studies $(1,2)$, confirmed that greater morbidity is seen following more extended procedures. In our opinion, the endoscopic removal of the arytenoid can be avoided in some cases, and lesions with moderate involvement of the arytenoid could be treated by a less aggressive procedure by sparing part of the inferior aspect of the arytenoid with significant improvement in 
swallowing recovery, while massive involvement of the arytenoid could be considered a contraindication for the endoscopic approach.

Primary elective neck dissection is not in contrast with the concept of lower morbidity of the endoscopic approach and, in the present study, increased regional control of the disease.

\section{REFERENCES}

1. Piazza C, Barbieri D, Del Bon F, Grazioli P, Perotti P, Paderno A, et al. Functional outcomes after different types of transoral supraglottic laryngectomy. Laryngoscope (2016) 126:1131-5. doi: 10.1002/lary.25562

2. Peretti G, Piazza C, Penco S, Santori G, Del Bon F, Garofolo S, et al. Transoral laser microsurgery as primary treatment for selected T3 glottic and supraglottic cancers. Head Neck (2016) 38:1107-12. doi: 10.1002/hed.24424

3. Remacle M, Hantzakos A, Eckel H, Evrard AS, Bradley PJ, Chevalier D, et al. Endoscopic supraglottic laryngectomy: a proposal for a classification by the working committee on nomenclature, European Laryngological Society. Eur Arch Otorhinolaryngol. (2009) 266:993-8. doi: 10.1007/s00405-008-0901-8

4. Roh JL, Kim DH, Park CI. Voice, swallowing and quality of life in patients after transoral laser surgery for supraglottic carcinoma. J Surg Oncol. (2008) 98:184-9. doi: 10.1002/jso.21101

5. Bernal-Sprekelsen M, Vilaseca-González I, Blanch-Alejandro JL. Predictive values for aspiration after endoscopic laser resections of malignant tumors of the hypopharynx and larynx. Head Neck (2004) 26:103-10. doi: 10.1002/hed.10363

6. Cabanillas R, Rodrigo JP, Llorente JL, Suárez V, Ortega P, Suárez C. Functional outcomes of transoral laser surgery of supraglottic carcinoma compared with a transcervical approach. Head Neck (2004) 26:653-9. doi: 10.1002/hed.20063

7. Puxeddu R, Sionis S, Gerosa C, Carta F. Enhanced contact endoscopy for the detection of neoangiogenesis in tumors of the larynx and hypopharynx. Laryngoscope (2015) 125:1600-6. doi: 10.1002/lary.25124

8. Amin MB, Greene FL, Edge SB, Compton CC, Gershenwald JE, Brookland RK, et al. The eighth edition AJCC CancerStaging Manual: continuing to build a bridge from a population-basedto a more "personalized" approach to cancer staging. CA Cancer J Clin. (2017) 67:93-9. doi: 10.3322/caac.21388

9. Jones TM, De M, Foran B, Harrington K, Mortimore S. Laryngeal cancer: United Kingdom National Multidisciplinary guidelines. J Laryngol Otol. (2016) 130:S75-82. doi: 10.1017/S0022215116000487

10. Roman BR, Goldenberg D, Givi B. AHNS Series-do you know your guidelines? Guideline recommended follow-up and surveillance of head and neck cancer survivors. Head Neck (2016) 38:168-74. doi: 10.1002/hed.24100

11. Dysphagia Section, Oral Care Study Group, Multinational Association of Supportive Care in Cancer (MASCC)/International Society of Oral Oncology (ISOO), Raber-Durlacher JE, Brennan MT, Verdonck-de Leeuw IM, et al. Swallowing dysfunction in cancer patients. Support Care Cancer (2012) 20:433-43. doi: 10.1007/s00520-011-1342-2

12. Alonso JM. Conservative surgery of cancer of the larynx. Trans Am Acad Ophthalmol Otolaryngol. (1947) 51:633-42.

13. Vaughan CW. Transoral laryngeal surgery using the $\mathrm{CO}_{2}$ laser: laboratory experiments and practice. Laryngoscope (1978) 88:1399-420. doi: 10.1002/lary.1978.88.9.1399

14. Puxeddu R, Pirri S, Bacchi PC, Salis G, Ledda GP. Endoscopic $\mathrm{CO}_{2}$ laser treatment of supraglottic carcinoma. Acta Otorhinolaryngol Ital. (2003) 23:459-66. doi: 10.1.1.550.5741\&rep=rep1\&type=pdf

15. Banko B, Djukic V, Milovanovic J, Kovac J, Novakovic Z, Maksimovic R. MRI in evaluation of neoplastic invasion into pre-epiglottic and paraglottic space. Auris Nasus Larynx (2014) 41:471-4. doi: 10.1016/j.anl.2014.02.008

16. Laccourreye O, Brasnu D, Biacabe B, Hans S, Seckin S, Weinstein G. Neo-adjuvant chemotherapy and supracricoid partial laryngectomy with

\section{AUTHOR CONTRIBUTIONS}

All authors contributed to the preparation of the manuscript. FC, CM, and RP wrote the manuscript. NC, EL, CM, and GS collaborated in the collection and analysis of the clinical data. CG was in charge of the pathologic analysis. All of the authors reviewed and contributed to the present form of the manuscript.

cricohyoidopexy for advanced endolaryngeal carcinoma classified as T3-T4: 5year oncologic results. Head Neck (1998) 20:595-9. doi: 10.1002/(SICI)10970347(199810)20:7<595::AID-HED3>3.0.CO;2-K

17. Peretti G, Piazza C, Ansarin M, De Benedetto L, Cocco D, Cattaneo $A$, et al. Transoral $\mathrm{CO}_{2}$ laser microsurgery for Tis-T3 supraglottic squamous cell carcinomas. Eur Arch Otorhinolaryngol. (2010) 267:1735-42. doi: 10.1007/s00405-010-1284-1

18. Rodrigo JP, Suárez C, Silver CE, Rinaldo A, Ambrosch P, Fagan JJ, et al. Transoral laser surgery for supraglottic cancer. Head Neck (2008) 30:658-66. doi: 10.1002/hed.20811

19. Esposito ED, Motta S, Cassiano B, Motta G. Occult lymph node metastases in supraglottic cancers of the larynx. Otolaryngol Head Neck Surg. (2001) 124:253-7. doi: $10.1067 / \mathrm{mhn} .2001 .113146$

20. Redaelli de Zinis LO, Nicolai P, Tomenzoli D, Ghizzardi D, Trimarchi M, Cappiello J, et al. The distribution of lymph node metastases in supraglottic squamous cell carcinoma: therapeutic implications. Head Neck (2002) 24:91320. doi: 10.1002/hed.10152

21. Lawson G, Matar N, Nollevaux MC, Jamart J, Krug B, Delos M, et al. Reliability of sentinel node technique in the treatment of N0 supraglottic laryngeal cancer. Laryngoscope (2010) 120:2213-7. doi: 10.1002/lary. 21131

22. Thompson CF, St John MA, Lawson G, Grogan T, Elashoff D, Mendelsohn AH. Diagnostic value of sentinel lymph node biopsy in head and neck cancer: a meta-analysis. Eur Arch Otorhinolaryngol. (2013) 270:2115-22. doi: 10.1007/s00405-012-2320-0

23. Rassekh $\mathrm{CH}$, Driscoll BP, Seikaly H, Laccourreye O, Calhoun KH, Weistein GS. Preservation of the superior laryngeal nerve in supraglottic and supracricoid partial laryngectomy. Laryngoscope (1998) 108:445-7. doi: 10.1097/00005537-199803000-00025

24. Rudert HH, Werner JA, Hoft S. Transoral carbon dioxide laser resection of supraglottic carcinoma. Ann Otol Rhinol Laryngol. (1999) 108:819-27. doi: $10.1177 / 000348949910800901$

25. Chiesa Estomba CM, Betances Reinoso FA, Lorenzo Lorenzo AI, Fariña Conde JL, Araujo Nores J, Santidrian Hidalgo C. Functional outcomes of supraglottic squamous cell carcinoma treated by transoral laser microsurgery compared with horizontal supraglottic laryngectomy in patients younger and older than 65 years. Acta Otorhinolaryngol Ital. (2016) 36:450-8. doi: 10.14639/0392-100X-864

26. Bertolin A, Lionello M, Russo S, Rizzotto G, Lucioni M. Could laser-assisted dissection of the pre-epiglottic space affect functional outcome after ESL? Laryngoscope (2017) 128:1371-8. doi: 10.1002/lary.26961

Conflict of Interest Statement: The authors declare that the research was conducted in the absence of any commercial or financial relationships that could be construed as a potential conflict of interest.

Copyright (c) 2018 Carta, Mariani, Sambiagio, Chuchueva, Lecis, Gerosa and Puxeddu. This is an open-access article distributed under the terms of the Creative Commons Attribution License (CC BY). The use, distribution or reproduction in other forums is permitted, provided the original author(s) and the copyright owner(s) are credited and that the original publication in this journal is cited, in accordance with accepted academic practice. No use, distribution or reproduction is permitted which does not comply with these terms. 


\section{Transoral Laser Microsurgery for Supraglottic Cancer}

\section{Petra Ambrosch ${ }^{1 *}$, Mireia Gonzalez-Donate ${ }^{1}$, Asita Fazel ${ }^{1}$, Claudia Schmalz ${ }^{2}$ and Jürgen Hedderich ${ }^{3}$}

${ }^{1}$ Department of Otorhinolaryngology, Head and Neck Surgery, Christian-Albrechts-University Kiel, Kiel, Germany, ${ }^{2}$ Department of Radiation Oncology, Christian-Albrechts-University Kiel, Kiel, Germany, ${ }^{3}$ Institute of Medical Informatics and Statistics, Christian-Albrechts-University Kiel, Kiel, Germany

Introduction: Transoral laser microsurgery (TLM) is an accepted and effective treatment strategy for supraglottic carcinomas. Early supraglottic carcinoma has excellent outcomes independently of the treatment approach. The role of TLM for the treatment of locally advanced tumors is debated. Particularly, the functional outcomes after TLM have to be proven by functional assessment of large cohorts of patients. This study analyzes the oncologic and functional outcomes after TLM for supraglottic carcinomas.

OPEN ACCESS

Edited by: Giorgio Peretti,

Università di Genova, Italy

Reviewed by: Jeroen Meulemans, University Hospitals

Leuven, Belgium

Giuseppe Mercante, National Cancer Institute

Regina Elena, Italy

*Correspondence:

Petra Ambrosch petra.ambrosch@uksh.de

Specialty section:

This article was submitted to Head and Neck Cancer, a section of the journal Frontiers in Oncology

Received: 26 February 2018 Accepted: 25 April 2018 Published: 09 May 2018

Citation:

Ambrosch P, Gonzalez-Donate $M$, Fazel A, Schmalz $C$ and Hedderich $J$ (2018) Transoral Laser Microsurgery for Supraglottic Cancer. Front. Oncol. 8:158. doi: 10.3389/fonc. 2018.00158
Patients and methods: Ninety-one patients with pT1-pT4a supraglottic carcinomas treated between January 2002 and December 2012 were analyzed. Distribution of tumors (UICC 2010) was 11 patients with pT1, 31 patients with pT2, 36 patients with pT3, and 13 patients with pT4a tumors. Node status was positive in 40 (43.6\%) patients; 61 (67.1\%) patients had stage III or IVa disease. Local control and survival were estimated using the Kaplan-Meier method. For the assessment of functional outcomes, the MD Anderson Dysphagia Inventory (MDADI), the Voice Handicap Index-10 (VHI-10), and the performance status scale for head and neck cancer [Performance Status Scale for Head and Neck (PSS-HN)] were used.

Results: The median age was 62 years (range, 33-88 years). Fourteen (15.4\%) patients developed a local or locoregional recurrence. The 5-year local control rate and 5-year ultimate local control rate were 72 and $92 \%$, respectively. The 5-year overall survival rate was 63\%. Twelve (13.2\%) patients needed temporary tracheostomy. Sixty-eight (74.0\%) patients had a nasogastric feeding tube post-operatively. At 1-year post-operative follow-up, only three patients were PEG dependent. The median VHI-10 score was 35 , the median MDADI composite score was 80 , and the median score of the domain "normalcy of diet" in the PSS-HN was 91.

Conclusion: The oncologic outcomes are comparable to the results of open surgery for early and advanced supraglottic carcinomas. Functional swallowing outcome is superior to open surgery and to concomitant chemoradiation. Patients treated with TLM perceive low levels of voice- and swallowing-related quality of life impairment.

Keywords: supraglottic cancer, transoral laser microsurgery, larynx preservation, oncologic outcomes, functional outcomes 


\section{INTRODUCTION}

Currently, different treatment modalities are available for supraglottic carcinomas. Oncologic outcome is still the most important treatment goal. However, the preservation of a functioning larynx and issues of quality of life (QoL) have become more important in recent years and influence decision-making. There is consensus that early supraglottic carcinomas can be effectively treated by open-neck supraglottic laryngectomy (SGL), transoral laser microsurgery (TLM), and radiotherapy (RT). The first long-term results of 240 patients with early laryngeal carcinomas - among them 30 patients with supraglottic lesions - treated with TLM between 1979 and 1985 were published by Steiner in 1993 (1). In a subsequent report, the validity of TLM for the treatment of early supraglottic carcinomas could be shown (2). In recent years, TLM has gained approval for organ preservation surgery in early supraglottic tumors as a less invasive surgical method with superior functional results compared to external partial laryngectomies. In a systematic review, Swanson et al. (3) found that TLM performed better than IMRT for the treatment of early supraglottic cancer. The use of TLM for surgical larynx preservation in locally advanced supraglottic carcinomas, however, is still discussed controversially. A limited number of cohort studies confirm the oncologic and functional benefits of $\operatorname{TLM}(2,4-14)$. With accurate preoperative diagnostics, appropriate patient selection, surgical expertise, and use of advanced technology adjuvant RT, TLM was shown to offer similar local control, larynx preservation, and survival as open-neck partial resections and similar survival as total laryngectomies. A recent meta-analysis of key oncological outcomes following TLM or open-neck conservation surgery for advanced T3-T4 laryngeal cancer confirmed both techniques as valid surgical options for larynx preservation (15). However, chemoradiotherapy protocols for larynx preservation have been examined in several randomized clinical trials (16-18) and a meta-analysis (19), thus making chemoradiotherapy an evidence-based and therefore preferred treatment approach in many cancer centers worldwide. However, recent studies have suggested that late toxicities are likely to contribute to high numbers of tumor-unrelated deaths years after treatment $(17,19)$. Another worrisome observation is that US cancer registry studies have shown that the increasing use of non-surgical larynx preservation strategies is accompanied by decreasing survival for patients with laryngeal cancer $(20,21)$. That fact was confirmed recently by data from Europe (22). Both observations raise the interest in larynx preservation surgery. The aim of this study is to present the oncologic and functional treatment results of TLM combined with selective neck dissection and adjuvant (chemo-)radiotherapy for early and locally advanced supraglottic carcinomas. The results are compared with results obtained with open-neck partial laryngeal resection and non-surgical larynx preservation with concurrent chemoradiotherapy.

\section{PATIENTS AND METHODS}

\section{Patients}

This study was approved by the ethics committee of the Medical Faculty of Kiel University (D 417/14). Between January 2002 and
December 2012, a total of 170 Patients with previously untreated squamous cell cancer of the supraglottic larynx were treated at the Department of Otorhinolaryngology, Head and Neck Surgery of Kiel University, Germany. Seventy-nine patients were excluded from the analysis with the following exclusion criteria: 17 patients presented with a previous malignant tumor, 9 patients were diagnosed with a simultaneous second primary tumor, 3 patients were diagnosed with simultaneous distant metastases, and 2 patients were diagnosed with non-resectable N3 metastases in the neck. Fifteen patients underwent concurrent chemoradiotherapy and another 22 patients were treated with total laryngectomy due to advanced local disease (T3 and T4a) with no option for non-surgical or surgical larynx preservation. Ten patients with very advanced locoregional disease were treated in palliative intent, and one patient was lost for follow-up. Ninetyone consecutively recruited patients were treated with frontline TLM. None underwent open-neck SGL, and none had to be excluded for technical reasons, due to insufficient endoscopic accessibility of the tumor. Of those 91 patients, $69(75.8 \%)$ patients were male and $22(24.2 \%)$ patients were female. The median age was 62 years (range, 33-88 years), and 17 (18.7\%) patients were older than 70 years. Seventy-nine $(86.8 \%)$ patients were active or former smokers with a median tobacco consumption of 35 pack years, $61(67.0 \%)$ patients consumed alcohol regularly, and 12 (13.2\%) patients were ex-alcoholics. Patient characteristics are shown in Table 1.

\section{Preoperative Examination}

Routine preoperative examination consisted in flexible laryngoscopy with adequate documentation. Accessibility of the tumor and tumor extent were determined with a staging microlaryngoscopy. All patients had panendoscopy for the exclusion of second primary tumors in the upper aerodigestive tract and a biopsy for tissue diagnosis. CT or MRI scans were routinely performed in all patients, except in T1 primary lesions. For staging of the neck, ultrasound and fine needle aspiration cytology were used, additionally to CT or MRI scans. Distant metastases were excluded by chest X-ray and ultrasonography of the abdominal organs. Pulmonary function tests were not routinely used.

\section{Operative Technique}

The operation was performed in the technique previously described $(2,23)$. Briefly, small lesions of the suprahyoid epiglottis or ventricular fold were excised en bloc. Carcinomas involving the infrahyoid epiglottis and the ventricular folds were exposed with a bivalved laryngoscope and removed piecemeal. In those cases, the preepiglottic fat was resected completely. Tumors with extension to the paraglottic space were removed with the resection of parts of the vocalis muscle. Tissues of the base of the tongue, piriform sinus, or one arytenoid cartilage were included in the resection if indicated. To prevent post-operative hemorrhage from the superior laryngeal arteries, the arteries were doubleclipped. In most cases, tracheostomy was not needed because of little post-operative edema. Tracheostomy was considered in elderly patients with low pulmonary reserve, in patients with a bleeding diathesis (e.g., anti-coagulant medication and 
TABLE 1 | Patient characteristics.

\begin{tabular}{|c|c|c|}
\hline Characteristic & $N$ & $\%$ \\
\hline \multicolumn{3}{|l|}{ Sex } \\
\hline Male & 69 & 75.8 \\
\hline Female & 22 & 24.2 \\
\hline \multicolumn{3}{|l|}{ pT category } \\
\hline pT1 & 11 & 12.0 \\
\hline pT2 & 31 & 34.0 \\
\hline рT3 & 36 & 39.5 \\
\hline pT4a & 13 & 14.5 \\
\hline \multicolumn{3}{|l|}{ pN category } \\
\hline NO/pNO & 51 & 56.0 \\
\hline pN1 & 9 & 10.0 \\
\hline $\mathrm{pN} 2 \mathrm{a}$ & 1 & 1.0 \\
\hline $\mathrm{pN} 2 \mathrm{~b}$ & 15 & 16.5 \\
\hline pN2c & 15 & 16.5 \\
\hline pN3 & 0 & 0 \\
\hline \multicolumn{3}{|l|}{ UICC stage } \\
\hline । & 8 & 8.8 \\
\hline ॥ & 22 & 24.2 \\
\hline III & 26 & 28.6 \\
\hline IVa & 35 & 38.5 \\
\hline \multicolumn{3}{|l|}{ Smoking status } \\
\hline Never & 12 & 13.2 \\
\hline Ever & 79 & 86.8 \\
\hline \multicolumn{3}{|l|}{ Alcohol consumption } \\
\hline Never & 19 & 20.8 \\
\hline Regular & 61 & 67.0 \\
\hline Ex-alcoholic & 12 & 13.2 \\
\hline \multicolumn{3}{|l|}{ Surgical margins } \\
\hline Negative (R0) & 82 & 90.1 \\
\hline Microscopic positive (R1) & 1 & 1.1 \\
\hline Uncertain & 8 & 8.8 \\
\hline Macroscopic positive (R2) & 0 & 0 \\
\hline
\end{tabular}

hemodialysis), or if bleeding from larger arteries was observed during surgery.

\section{Treatment of the Primary Tumor}

All patients received TLM with the intent to completely remove the supraglottic carcinoma with microscopically negative margins (R0 resection). The resection was guided by the growth of the tumor. Resections were not classified according to the ELS classification system for supraglottic resections (24). The reasons are that despite the complexity of the classification system, not all resections are classifiable and that the system is not yet widely accepted. In 25 (27.5\%) patients, a re-resection was performed because of positive ( $\mathrm{R} 1)$ or uncertain $(\mathrm{Rx})$ resection margins. Only four of the 25 re-resection specimens contained carcinoma cells. Including re-resection, $78(85.7 \%)$ of the 91 supraglottic carcinomas were resected with clear margins. Thirteen $(14.3 \%)$ patients had microscopically positive $(n=5)$ or uncertain margins $(n=7)$. Ten of those had adjuvant (chemo-)radiotherapy, and three patients were clinically controlled. Five (5.5\%) patients were prophylactically tracheotomized after extended supraglottic resection to secure the airway and to reduce the risk of developing pneumonia. All patients were decannulated.
TABLE 2 | Treatment approach.

\begin{tabular}{|c|c|c|c|c|c|}
\hline \multirow[t]{2}{*}{ UICC stage } & \multicolumn{2}{|c|}{$\begin{array}{c}\text { Surgical } \\
\text { treatment }\end{array}$} & \multicolumn{2}{|c|}{$\begin{array}{l}\text { Combined } \\
\text { treatment }\end{array}$} & \multirow[t]{2}{*}{ Total } \\
\hline & $N$ & $\%$ & $N$ & $\%$ & \\
\hline 1 & 8 & 8.8 & 0 & & 8 \\
\hline$\|$ & 19 & 20.9 & 3 & 3.3 & 22 \\
\hline III & 19 & 20.9 & 7 & 7.7 & 26 \\
\hline \multirow[t]{2}{*}{$\mathrm{IVa}$} & 15 & 16.4 & 20 & 22.0 & 35 \\
\hline & 61 & 67.0 & 30 & 33.0 & 91 \\
\hline
\end{tabular}

Pathologic staging according to the UICC 2010 classification was used for the primary tumor and the neck. The distribution of the histopathologic grading of the primary tumors was as follows: G1, 7.0\%; G2, 68\%; G3, 25\%.

\section{Treatment of the Neck}

Seventy-six $(83.5 \%)$ patients had either unilateral $(34 ; 37.4 \%)$ or synchronous bilateral $(42 ; 46.2 \%)$ neck dissection. In clinically node-negative necks, selective neck dissection with removal of the lymph nodes of levels II and III and in clinically node-positive necks of levels II-IV was performed. Neck node metastases were histopathologically confirmed in 40 (44.0\%) patients. Metastases with extranodal spread were detected in 15 of the 40 (37.5\%) patients with node-positive necks.

\section{Adjuvant Treatment}

Adjuvant RT was not indicated in patients with R0 resected primary tumors and with pN0 necks. Adjuvant RT was indicated in patients with microscopically positive resection margins (R1 resection), in patients with more than one lymph node metastases in the neck and/or metastases with extranodal spread. Radiation technique and chemotherapy changed over time. In the vast majority of patients, a conventional radiation therapy technique was used ("Fletcher-technique" with three-dimensional planning, $6-\mathrm{MeV}$ photons of a linear accelerator). In all cases, post-operative RT was directed to the primary site and both sides of the neck.

Post-operative RT was performed in 30 (32.9\%) patients, $18(60.0 \%)$ of whom also received concurrent platinum-based chemotherapy. Adjuvant (chemo-)radiotherapy was performed in $3 / 30(10 \%)$ patients in stages I and II and in 27/61 (44.2\%) patients in stages III and IVa because of positive resection margins, more than one lymph node metastases in the neck or lymph node metastases with extranodal spread (Table 2). Extranodal spread of lymph node metastases and positive resection margins were regarded indications for adjuvant chemoradiotherapy. Chemoradiotherapy received 18 out of 19 patients with positive margins and/or ENS. Multiple neck node metastases and uncertain margins were not regarded indications for chemoradiotherapy. Treatment consisted in 60 Gy to the primary site after R0 resection and in $64 \mathrm{~Gy}$ after $\mathrm{R} 1$ resection and in patients with uncertain resection margins. The radiation dose to the node-negative neck (N0/pN0) was $50 \mathrm{~Gy}$, to the node-positive neck $\left(\mathrm{pN}^{+}\right) 54 \mathrm{~Gy}$. Radiation dose was conventionally fractionated (2 Gy once daily, total treatment time, 6 weeks). Chemotherapy was carboplatin 
$\left(70 \mathrm{mg} / \mathrm{m}^{2} /\right.$ week $)$ in 14 patients or cisplatin $\left(25 \mathrm{mg} / \mathrm{m}^{2}, \mathrm{~d} 1-\mathrm{d} 4\right.$, weeks 1,3 , and 6) in 4 patients.

\section{Assessment of Swallowing- and Voice-Related QoL}

The MD Anderson Dysphagia Inventory (MDADI) was used for the assessment of swallowing-related QoL. The MDADI is validated for German language and is a self-administered questionnaire with 20 questions for the evaluation of the patient's perception of swallowing-related QoL. Besides a global assessment (a single question), it comprises the following three subscales: physical subscale (six items), functional subscale (five items), and emotional subscale (eight items). The instrument was scored according the index paper (25). The minimum composite score is 20 (extremely low functioning), and the maximum composite score is 100 (high functioning). Thus, a higher MDADI composite score represents better function and better QoL. The Voice Handicap Index (VHI) was used for the assessment of voicerelated QoL. The VHI questionnaire is also validated for German language and comprises 30 items in three subscales (physical, functional, and emotional). The instrument was scored according the index paper (26). Using a 5-point scale, the maximum score is 120 (extremely low functioning). Thus, a low VHI score represents better function and better QoL. The Performance Status Scale for Head and Neck (PSS-HN) was used for the assessment of subjective swallowing function (27). The PSS-HN is an examiner-rated instrument. The subscales "normalcy of diet," "understandability of speech," and "eating in public" are rated from 0 to 100, with higher scores indicating better performance. Functional morbidities with score $\leq 50$ were considered as significant (28).

In April 2014, a cross-sectional study on patient-reported outcomes was performed. Forty-six patients were still alive. Five patients were excluded, three patients because of previous laryngectomy due to recurrence $(n=2)$ or functional failure $(n=1)$ and two patients because of permanent PEG feeding. Those five patients were regarded as functional failures with respect to the SGL, initially performed. Forty-one patients received the MDADI and VHI questionnaires. The questionnaires were handed out to the patients in the outpatient clinic, or mailed with a personalized cover letter. Patients were asked to complete and return the questionnaires. The questionnaires were completed by $28(68.3 \%)$ patients (VHI) and $29(70.7 \%)$ patients (MDADI). For functional assessment with the PSS-HN, $36(87.8 \%)$ patients were interviewed by the treating clinician in the outpatient clinics.

\section{Statistical Methods}

Data were recorded prospectively and kept in a database. Follow-up data were available of all patients. The median followup interval was 47.4 months (range, 2.5-124.7 months). Local control and survival were calculated according the Kaplan-Meier method. All data of patient-reported outcomes (MDADI, VHI, and PSS-HN scores) were expressed descriptively, compared between subgroups and displayed with box-whisker plots. Statistical tests were not used because of limited numbers in subgroups.

\section{RESULTS}

\section{Local Control}

Fourteen (15.4\%) patients developed local (eight patients) or locoregional (six patients) recurrences. Nine patients were successfully salvaged: seven patients with further TLM (2 with TLM + ND, one patient with TLM + RT), and two patients with total laryngectomy; five patients are alive and free of tumor; and two patients died from tumor-unrelated causes. In three patients, salvage therapy (two patients with total laryngectomy and one patient with definitive RT) was not successful and two patients declined further surgery or RT and were treated with palliative intent. The analysis of local and locoregional recurrences is shown in Table 3. The 5-year Kaplan-Meier local control rate was $72 \%$ [62\%; 83\%] for the entire cohort, $76 \%$ for pT1 and pT2, 68\% for $\mathrm{pT} 3$, and $67 \%$ for $\mathrm{pT} 3$ and $\mathrm{pT} 4 \mathrm{a}$ diseases. The 5 -year definitive local control rate (including salvage therapy) was $92.3 \%$ [87\%; $100 \%$ ] for the entire cohort. The Kaplan-Meier estimates of local control and the corresponding 95\% confidence intervals are shown in Table 4.

\section{Larynx Preservation}

In seven $(7.7 \%)$ patients, larynx preservation failed because of oncologic, and in three (3.3\%) patients, larynx preservation failed because of functional reasons. In $81 / 91$ (89\%) patients,

TABLE 3 | Analysis of local and locoregional recurrences.

\begin{tabular}{lclll}
\hline TNM & $\begin{array}{c}\text { Time since } \\
\text { surgery } \\
\text { (months) }\end{array}$ & rTrN & $\begin{array}{l}\text { Salvage } \\
\text { treatment }\end{array}$ & Outcome \\
\hline pT1pN0 & 16 & $r T 1$ & TLM & Alive, NED \\
pT1pN0 & 87 & $r T 1$ & TLM & Alive, NED \\
pT1pN0 & 63 & $r T 1$ & TLM & Alive, NED \\
pT2 pN0 & 15 & $r T 2$ & TLM & Alive, NED \\
pT2 pN0 & 11 & $r T 4$ & TL & Alive, NED \\
pT3pN0 & 56 & $r T 2$ & TLM + RT & Dead, tumor unrelated \\
pT3pN0 & 23 & $r T 4$ & TL + RT & Alive, NED \\
pT3pN1 & 8 & $r T 4$ & TL & Dead, tumor related \\
pT2pN0 & 20 & $r T 1 r N 1$ & TLM + ND & Dead, tumor unrelated \\
pT3pN2a & 10 & $r T 4 r N 2 c$ & RT & Dead, tumor related \\
pT3pN0 & 23 & $r T 1 r N 1$ & TLM + ND & Alive, NED \\
pT3pN0 & 10 & $r T 4 r N 2 c$ & Declined & Dead, tumor related \\
pT4apN0 & 24 & $r T 4 r N 2 c$ & TL + RT & Dead, tumor related \\
pT4apN2c & 6 & $r T 2 r N 2 c$ & Declined & Dead, tumor related \\
\hline
\end{tabular}

TABLE 4 | Kaplan-Meier estimates of local control.

\begin{tabular}{lccc}
\hline & Years & Local control rate (\%) & 95\% confidence interval \\
\hline pT1 and pT2 & 2 & 83 & $72 ; 96$ \\
& 3 & 83 & $72 ; 96$ \\
pT3 & 5 & 76 & $63 ; 92$ \\
& 2 & 72 & $58 ; 90$ \\
pT3 and pT4a & 3 & 72 & $58 ; 90$ \\
& 5 & 68 & $54 ; 87$ \\
& 3 & 75 & $63 ; 89$ \\
& 5 & 67 & $63 ; 89$ \\
& 5 & & $56 ; 85$
\end{tabular}


a functioning larynx could be preserved. The larynx could be preserved in 10/11 (91\%) patients with pT1, in 30/31 (97\%) patients with pT2, in 32/36 (89\%) patients with pT3, and in 9/13 (69\%) patients with pT4a primary tumors.

\section{Regional Control}

The overall recurrence rate in the neck was 5.5\% (5/91 patients). Three (3.3\%) patients developed late metastases in the pN0 neck: two patients were successfully salvaged and one patient died tumor related. Two (2.2\%) patients developed recurrent neck metastases: one patient was successfully salvaged and one patient declined further treatment and died from neck recurrence.

\section{Distant Metastases}

Six (6.6\%) patients developed distant metastases (five patients in the lung and one patient in lung and liver) without local or neck recurrence after median 10 months (range, 1-17 months) after surgery. All patients, who developed distant metastases, had advanced neck disease at presentation (five patients $\mathrm{pN} 2 \mathrm{c}$ and one patient $\mathrm{pN} 2 \mathrm{~b}$ ). In none of the patients with local or locoregional recurrences, distant metastases have been diagnosed.

\section{Second Primary Tumors}

Second primary tumors were diagnosed in 17 (18.7\%) patients. The second primary tumor occurred in the head and neck region in five (vocal cord contralateral to supraglottic carcinoma, one; tonsil, three; outer ear canal, one) and in other organs in 12 (lung, seven; esophagus, one; thyroid gland, one; colon, two; urinary bladder, one) cases.

\section{Survival}

At the end of follow-up, 46 patients were alive and tumor-free. Fourteen (15.4\%) patients had died from cancer and $31(34.0 \%)$ patients had died from tumor-unrelated causes, eight $(8.8 \%)$ of whom due to second primary tumors. The 5-year Kaplan-Meier overall survival rate was $69 \%$ for stages I and II and $58 \%$ for stages III and IVa diseases. The 5-year Kaplan-Meier disease-free survival rate was $79 \%$ for stages I and II and $64 \%$ for stages III and IV diseases. The Kaplan-Meier estimates for overall and disease-free survival and the corresponding 95\% confidence intervals are shown in Table 5 for stages I and II and in Table 6 for stages III and IVa diseases.

\section{Post-operative Complications}

Two (2.2\%) patients experienced post-operative endolaryngeal hemorrhage. In both cases, the bleeding vessel was coagulated

TABLE 5 | Kaplan-Meier estimates of survival for stages I and II.

\begin{tabular}{lccc}
\hline & Years & Survival rate (\%) & 95\% confidence interval \\
\hline Overall survival & 2 & 90 & $80 ; 100$ \\
& 3 & 78 & $64 ; 96$ \\
Disease-free survival & 5 & 69 & $53 ; 90$ \\
& 2 & 85 & $72 ; 100$ \\
& 3 & 85 & $72 ; 100$ \\
& 5 & 79 & $65 ; 98$
\end{tabular}

endoscopically and one of whom was tracheotomized. Four (4.4\%) patients developed aspiration pneumonia, two of whom were tracheotomized. Another four (4.4\%) patients suffered from airway-compromising laryngeal edema and were tracheotomized to secure the airway. Altogether, a total of 12 (13.2\%) patients had temporary tracheostomies. In five patients tracheostomy was performed electively and in seven patients due to various complications, listed earlier. There were no therapy-related deaths and no permanent tracheostomies.

\section{Functional Results \\ Swallowing Rehabilitation}

Twenty-three (26\%) patients needed no feeding tube, among them 18 of the $42(43 \%)$ patients with pT1 and pT2 and five of the $49(10 \%)$ patients with pT3 and pT4a primary tumors. The remaining $68(74 \%)$ patients received a feeding tube at the end of surgery. The feeding tube was removed when no aspiration was endoscopically visible, median after 14 days. Two (2.2\%) patients remained dependent on PEG feeding and one (1.1\%) patient had a total laryngectomy for functional reasons.

\section{Dysphagia-Related QoL}

To evaluate patients' account of their long-term swallow function, 41 patients alive and free of recurrence received the MDADI questionnaire. The questionnaire was fully completed by 29 patients. The median interval between completion of therapy and assessment was 88.5 months (range, 25-138 months; median: 75.7 months). The median MDADI composite score in the whole patient group was 80 (range, 30-100). Subgroup analysis showed that the parameters size of the primary tumor (pT1/pT2 vs. pT3/ pT4a) had no relevant influence on the MDADI composite score, with 90 vs. 80. Relevant influence, however, had post-operative RT with a median MDADI composite score of 70 in irradiated and 90 in non-irradiated patients (Figure 1).

The PSS-HN instrument was completed for 36 patients. The median score for normalcy of diet was 91, that for eating in public was 85 , and that for the understandability of speech was 90 . The prevalence of functional deficits (score $\leq 50$ ) was 8,11 , and $5 \%$.

The VHI questionnaire was completed by 28 patients. The total VHI score in the whole patient group was 35 (range, 0-114). Few patients perceived high levels of voice handicap. Subgroup analysis showed that the parameters size of the primary tumor (pT1/pT2 vs. pT3/pT4a) had no relevant influence, with a median score of 23 vs. 37. Relevant influence, however, had post-operative RT with a median VHI score of 49 in irradiated and 16 in non-irradiated patients (Figure 2).

TABLE 6 | Kaplan-Meier estimates of survival for stages III and IVa.

\begin{tabular}{lccc}
\hline & Years & Survival rate (\%) & 95\% confidence interval \\
\hline Overall survival & 2 & 75 & $65 ; 87$ \\
& 3 & 68 & $57 ; 81$ \\
Disease-free survival & 5 & 58 & $47 ; 73$ \\
& 3 & 71 & $59 ; 84$ \\
& 5 & 69 & $57 ; 82$ \\
& 54 & $52 ; 78$
\end{tabular}




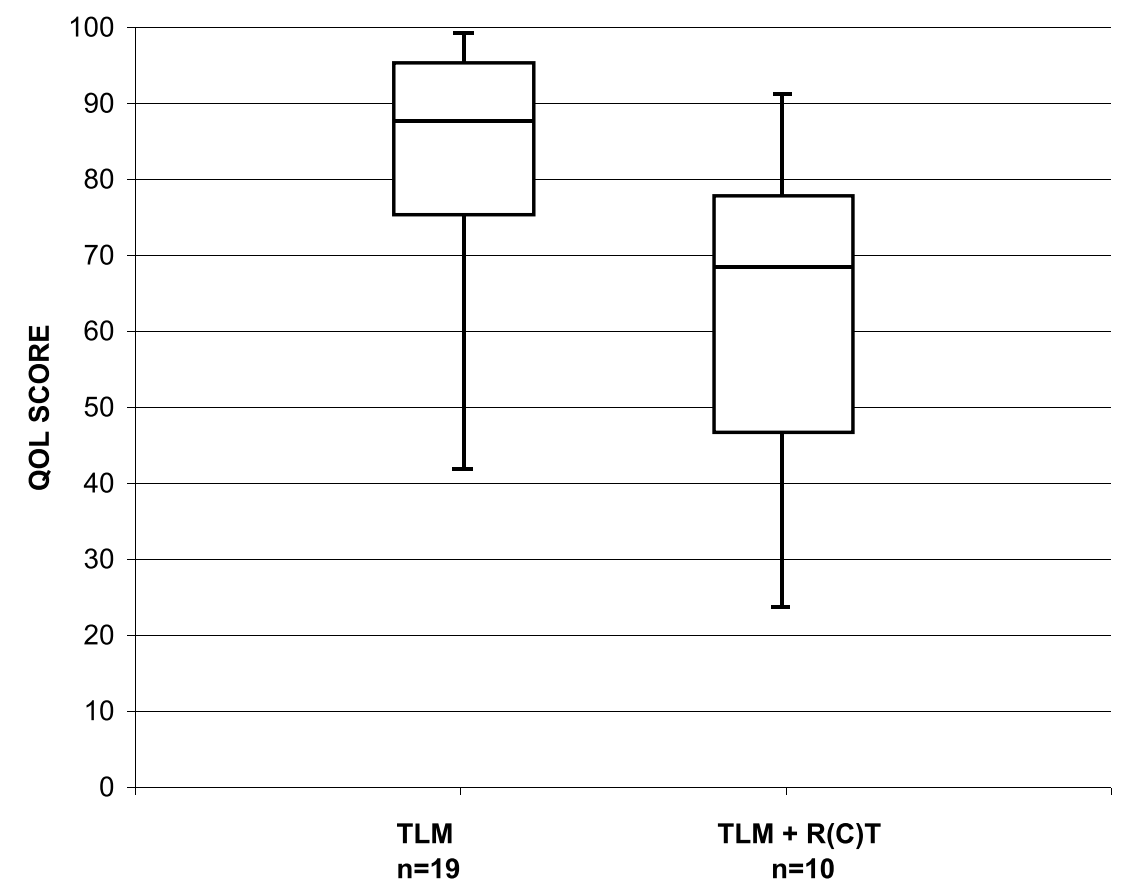

FIGURE 1 | Distribution of the MDADI composite score in long-term survivors after TLM $(n=19)$ and after TLM + adjuvant RT $(n=10)$.

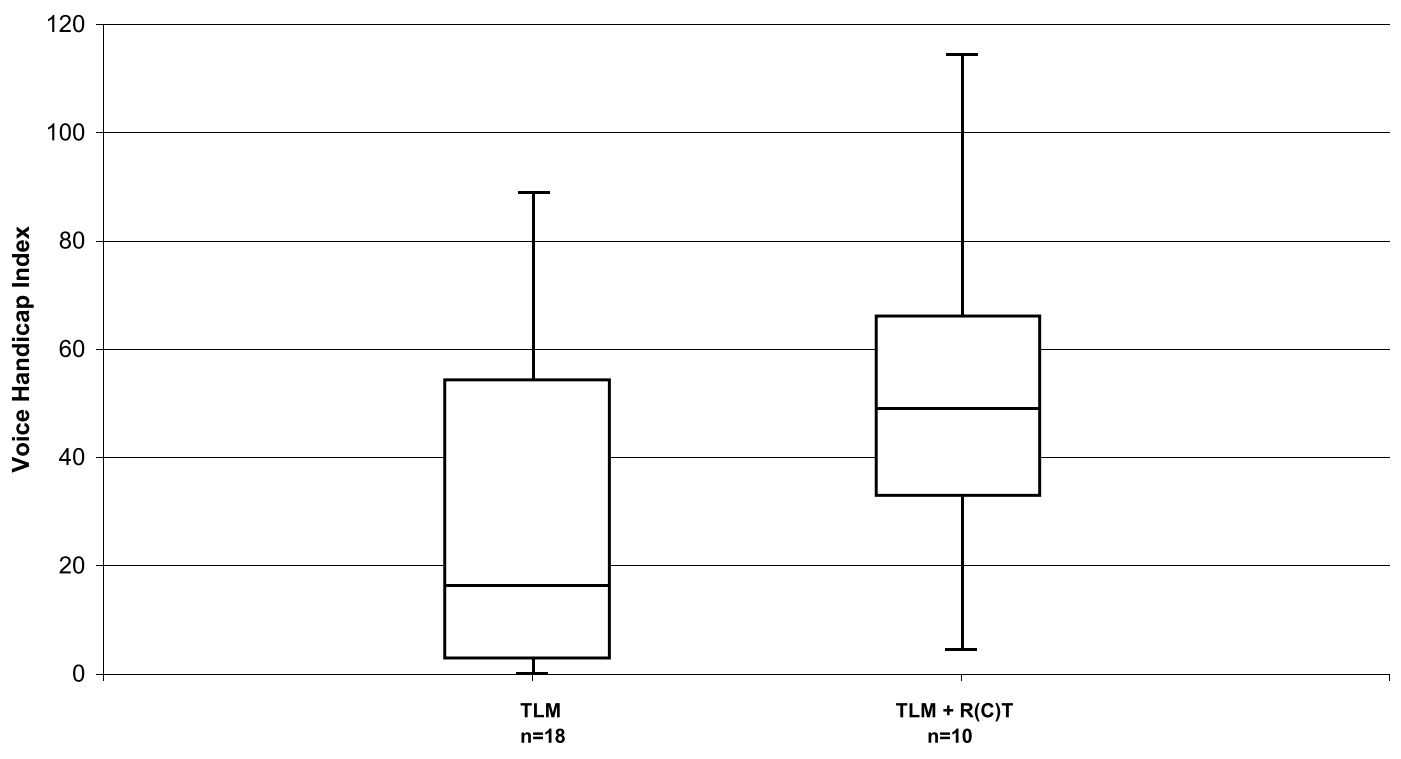

FIGURE 2 | Distribution of the VHI in long-term survivors after TLM $(n=18)$ and after TLM + adjuvant RT $(n=10)$.

\section{DISCUSSION}

Since a national clinical practice guideline on diagnostic and treatment of laryngeal carcinoma was not available at the time, patients were treated, the treatment decisions were made in concordance with institutional guideline recommendations accepted at that time. From today's knowledge, the potential value of adjuvant chemoradiotherapy in head and neck cancer patients with an elevated risk of recurrence is acknowledged and the modalities of adjuvant treatments have changed (29-31) also in our clinical practice.

It is generally agreed, however, that for the treatment of early supraglottic carcinomas (T1N0 and T2N0) single-modality therapy with the goal of curing the disease and preserving laryngeal functions is recommended. Early supraglottic carcinomas can be treated with RT or partial laryngeal resection with similar 
survival results (18). The surgical treatment options include SGL, either open-neck or TLM for the treatment of the primary tumor, together with uni- or bilateral, selective neck dissection. The non-surgical treatment is RT, preferably as IMRT, as single modality. Multimodality treatment with surgery and RT should be avoided because of added toxicity, poorer functional results, and increased necessity of total laryngectomy for salvage in the event of recurrence. In clinical practice, however, adjuvant RT in early stages is not always avoidable. In our cohort, three of the $30(10 \%)$ patients with early supraglottic carcinomas had combined treatment because of positive or uncertain resection margins with no option for re-resection.

In selected cases of locally advanced supraglottic carcinomas (T3/T4a N0/N+), surgical larynx preservation, either by TLM or by open-neck SGL is an option. Contraindications for open-neck and endoscopic SGL in T3/T4a supraglottic carcinomas are bilateral paraglottic space invasion, bilateral vocal cord fixation, invasion of the thyroid cartilage at the glottic level, and extensive infiltration of the base of tongue, piriform sinus or soft tissues of the neck. With limited disease in the neck, the surgical treatment of the regional lymphatics consists in bilateral selective neck dissection. Depending on the histopathologic findings, regarding resection margins and status of the neck nodes, adjuvant radio- or chemoradiotherapy of the larynx and the neck might be indicated. In our patient cohort, 27 of the $61(44.3 \%)$ patients with locally advanced supraglottic carcinomas needed combined treatment. The prevalence of adjuvant radio- and chemoradiotherapy varies among published TLM series between 22 and $41 \%$ and is mostly reported for the total patient cohort treated $(8-10,12,14,32)$.

\section{Oncologic Outcomes}

There is evidence from cohort studies that the oncologic outcomes following TLM for early supraglottic carcinoma are comparable to open-neck SGL and to RT. The 5-year local control rate was $76 \%$ for $\mathrm{pT} 1 / \mathrm{pT} 2$ tumors in our cohort. A high larynx preservation rate of $91 \%$ for $\mathrm{pT} 1$ and $97 \%$ for $\mathrm{pT} 2$ carcinomas could be achieved by early detection of local recurrences, which could be treated in five of the six cases by further larynx preserving TLM. The 5-year overall and disease-free survival rates for stages I and II were 69 and 79\%, respectively. These results are comparable to other TLM series.

In a previous report, Ambrosch et al. (2) achieved a 5-year local control rate of $100 \%$ in pT1 and $89 \%$ in pT2 supraglottic tumors. Iro et al. (4) found 5-year local control rates of $95 \%$ for stage I and $88 \%$ for stage II disease. Grant et al. (5) reported on 38 patients with mainly early supraglottic carcinomas. The 2 -year local control rate was 97 and $79 \%$ patients retained the larynx. Agrawal et al. (6) published a prospective phase 2 study in which 34 patients with T1/T2 supraglottic carcinomas were treated with TLM and post-operative RT. Only one patient was laryngectomized due to recurrence, but $9 \%$ failed because of functional reasons. Canis et al. (10) treated 277 patients with supraglottic carcinomas of all stages with TLM, among then 118 patients with $\mathrm{pT} 1 / \mathrm{pT} 2$ lesions. The 5-year local control rate was $85 \%$. Overall and recurrence-fee survival rates for stages I and II were 76 and $81 \%$. Peretti et al. (8) achieved in 58 patients with pTis-pT2 disease a 5-year larynx preservation rate of $100 \%$.

Two studies compare retrospectively open-neck SGL with TLM. In the series of Bussu et al. (7), larynx preservation and survival showed no significant difference in between treatment options, whereas functional results were better after TLM. Karatzanis et al. (33) compared oncologic and functional results of patients with early supraglottic carcinomas after TLM, openneck SGL, or total laryngectomy. Local control and diseasespecific survival were not significantly different. Functional outcomes were slightly better in the TLM group.

In our cohort, the 5-year local control rate was $68 \%$ for pT3 and $67 \%$ for pT3 and pT4a diseases. A functioning larynx could be preserved in $89 \%$ of patients with pT3 and in $69 \%$ patients with pT4a primary tumors. In the event of local recurrence, the auspices for salvage are compromised. Salvage treatment was successful in only two of the eight local/locoregional recurrences.

There are fewer reports on TLM for locally advanced supraglottic carcinomas in the literature. In the series of Iro et al. (4), local recurrence was diagnosed in 33\% patients with T3 and in $10 \%$ patients with T4 carcinomas. Ambrosch et al. (34) treated 50 patients with pT3 supraglottic carcinomas with TLM. The 5 -year recurrence-free survival and larynx preservation rates were 71 and $96 \%$, respectively. Peretti et al. (8) reported on 20 patients with pT3 supraglottic carcinomas. The 5-year local control rate was $83 \%$. In Vilaseca et al.'s (13) series of patients with T3 supraglottic carcinomas, the 5-year local control, overall, and disease-specific survival rates were 70,46 , and $62 \%$, respectively. Steiner's group reported in a retrospective review, long-term results of 104 pT3 and 55 pT4a patients with supraglottic carcinomas treated with TLM. Local or locoregional recurrences were observed in $20 \%$ of pT3 and in $22 \%$ of pT4a cases. The 5 -year larynx preservation rate was $82 \%$ for $\mathrm{pT} 3$ and $76 \%$ for pT 4 a carcinomas. The 5-year overall, recurrence-free, and disease-specific survival rates for stages III and IVa were 59, 65, and 81\% (11). Pantazis et al. (12) achieved with TLM in 24 patients with pT3 supraglottic carcinomas a 5 -year disease-specific survival and larynx preservation rate of $92 \%$. Vilaseca et al. (14) published the largest series of 128 patients with pT3 and 25 patients with pT4a supraglottic carcinomas treated with TLM. The 5-year laryngectomy-free survival was $75 \%$. The 5 -year overall survival rate was $56 \%$.

In the recent literature, only a few publications can be found on oncologic results of open-neck SGL and supracricoid partial laryngectomy for supraglottic carcinomas. For open-neck SGL in T1-T3 supraglottic carcinomas, 5-year local control rates from 90 to $93 \%$ and overall survival rates from 52 to $75 \%$ have been reported (35-37). Approximately 10\% patients need secondary laryngectomy because of aspiration (37). With extended openneck SGL for T3 carcinomas, local control rates from 71 to $94 \%$ have been published $(38,39)$. Schwaab et al. (40) reported on a larger series of patients, mainly with T2 and T3 tumors, who had supracricoid partial resection with cricohyoidopexy. Only $4 \%$ patients experienced local recurrence. Post-operative aspiration was a relevant problem in 19\% patients, and 9\% needed laryngectomy for functional reasons. The 5-year overall survival rate was $88 \%$. In summary, it could be shown that the oncologic 
results achieved by us are comparable to other TLM series, to open-neck SGL and to supracricoid partial resection.

In 2003, the RTOG 91-11 trial showed better locoregional control and larynx preservation for concurrent chemoradiotherapy than for induction chemotherapy followed by RT and by RT alone (16). Since that time, in many cancer centers, concurrent chemoradiotherapy has become the preferred nonsurgical treatment for larynx preservation in locally advanced supraglottic carcinomas, despite its toxicity. Even though more than $80 \%$ of the patients in the RTOG 91-11 trial had a Karnofsky index of $>90$, only $70 \%$ of patients could complete the concurrent chemoradiotherapy protocol due to toxicity. A high number of patients (82\%) suffered from severe toxicity, and $5 \%$ died of therapy-associated complications. Thus, therapy-related mortality is significantly higher when concurrent chemoradiotherapy is used for larynx preservation compared to the surgical approaches. Long-term results of the RTOG 91-11 trial were published in 2013 (17). It was remarkable that in the concurrent chemoradiotherapy arm of the trial, late deaths unrelated to larynx cancer occurred. The cause of death in these patients is not known and it was speculated that some might be due to late-toxicity such as swallowing dysfunction and (silent) aspiration (17).

\section{Complications}

For the evaluation of a surgical procedure, the complication rate is an important factor. In our series, $2.2 \%$ patients experienced post-operative endolaryngeal hemorrhage, requiring coagulation in general anesthesia. The incidence of post-operative hemorrhage is reported between 4 and $13 \%$ in different TLM series $(9,10,12-14)$. The lower incidence experienced by us is attributed to consequent liger clipping of both supralaryngeal arteries. Another complication occurring frequently after supraglottic resection is aspiration pneumonia. In our cohort, the incidence was $4 \%$. In other TLM series, the incidence varies between 2 and $12 \%(9,10,13,14,41)$. The risk of aspiration after open-neck SGL and supracricoid partial resection is even higher. The reported aspiration risk ranges from 3.8\% to $19 \%$ for open-neck surgery $(35,37-40)$. After supracricoid partial resection, the risk of severe aspiration followed by lung complications increases with increasing age and pre-existing lung disease. This explains why patients older than 60 years are often excluded from supracricoid partial resection with cricohyoidopexy (42).

In our patient cohort, a total of $12(13.2 \%)$ patients had temporary tracheostomy; five patients were tracheotomized electively and seven patients due to various complications. The incidence of temporary tracheostomy in different TLM series varies between 4 and $45.8 \%(8-10,12)$. This variation may reflect different patient populations and different indications for prophylactic tracheostomy. The prevalence of permanent tracheostomy is reported below $3 \%(9,10,12,14)$.

\section{Functional Outcomes}

A functioning larynx could be preserved in 89\% (81/91) patients in our cohort. According to T category, larynx preservation was possible in $91 \%$ of patients with pT1, 97\% of patients with pT2,
$89 \%$ of patients with pT3, and $69 \%$ of patients with pT4a tumors. At the end of follow-up, no patient had a tracheostoma, two patients needed permanent PEG feeding (those patients declined laryngectomy), and one patient underwent total laryngectomy because of severe aspiration. All authors agree that swallowing rehabilitation is quicker and better following TLM than openneck SGL (42). The reported rate of secondary laryngectomies in TLM series is $0-4 \%$, and the rate of permanent PEG feeding is $0-2 \%(9,10,12,14,43)$. The frequency of laryngectomy for persistent aspiration after open-neck SGL ranges between 3.5 and $12.5 \%(42)$.

\section{Patient-Reported Outcomes}

Quality of life questionnaires are routinely used in clinical trials. In contrast, it is uncommon to ascertain QoL data in clinical practice. Since our patient cohort was not treated in a prospective trial, baseline functional assessments preoperatively were not done. We performed a cross-sectional study and administered three QoL instruments to the disease-free long-term survivors of our cohort. The study was done median 88.5 months (range, 25-135 months) after completion of the cancer treatment. Normalcy of diet has the highest magnitude for patients, and our cohort achieved excellent outcomes with a median score of 91 . However, three of the 36 (8.3\%) patients perceived unsatisfactory outcomes with a PSS-HN score of 50. Restrictions in the domain "normalcy of diet" were due to discomfort caused by xerostomia. The dysphagia-related and voice-related QoL was examined with post-treatment MDADI and VHI scores. The median scores for our group of long-term survivors indicated that patients perceive low levels of swallowing and voice impairment. We could see, however, a clearly negative influence of post-operative RT on both swallowing and voice-related QoL. Today, the standard technique for adjuvant RT is IMRT and better preservation of the salivary glands and reduction of the radiation dose to uninvolved swallowing structures hopefully will translate in better swallowing- and voice-related QoL.

\section{Limitations}

The present study has some limitations. First, this study is a retrospective outcomes analysis, although the data have been recorded prospectively. In addition, the study included a limited number of patients, particularly the cross-sectional study of swallowing- and voice-related QoL. The fact that QoL could only be examined in the highly selected subgroup of long-term survivors could be a potential selection bias. In order to address these limitations, additional studies with larger sample-size are needed to more definitely assess oncologic and functional outcomes of TLM vs. alternative treatment strategies.

\section{Conclusion}

In conclusion, our results suggest that TLM can be considered an option for surgical larynx preservation in early and selected locally advanced supraglottic carcinomas. The oncologic results are comparable to open-neck SGL \pm adjuvant (chemo-)radiotherapy and to definitive chemoradiotherapy. A functioning larynx can be preserved in high numbers of patients. Tracheostomy and gastrostomy can be avoided in most cases. Patient-reported 
outcomes demonstrate that long-term survivors perceive low levels of QoL impairment and functional deterioration over time does not occur.

\section{ETHICS STATEMENT}

The study was approved by the Ethics Committee of the Medical Faculty of the Christian-Albrechts-University of Kiel, Number D $417 / 14$

\section{REFERENCES}

1. Steiner W. Results of curative laser microsurgery of laryngeal carcinomas. Am J Otolaryngol (1993) 14:116-21. doi:10.1016/0196-0709(93)90050-H

2. Ambrosch P, Kron M, Steiner W. Carbon dioxide laser microsurgery for early supraglottic carcinoma. Ann Otol Rhinol Laryngol (1998) 107:680-8. doi:10.1177/000348949810700810

3. Swanson MS, Low G, Sinha UK, Kokot N. Transoral surgery vs intensitymodulated radiotherapy for early supraglottic cancer: a systematic review. Curr Opin Otolaryngol Head Neck Surg (2017) 25:133-41. doi:10.1097/MOO. 0000000000000345

4. Iro H, Waldfahrer F, Altendorf-Hofmann A, Weidenbecher M, Sauer R, Steiner W. Transoral laser surgery of supraglottic cancer. Arch Otolaryngol Head Neck Surg (1998) 124:1245-50. doi:10.1001/archotol.124.11.1245

5. Grant DG, Salassa JR, Hinni ML, Pearson BW, Hayden RE, Perry WC. Transoral laser microsurgery for carcinoma of the supraglottic larynx. Otolaryngol Head Neck Surg (2007) 136:900-6. doi:10.1016/j.otohns.2006.12.015

6. Agrawal A, Moon J, Davis RK, Sakr WA, Giri SPG, Valentino J, et al. Transoral carbon dioxide laser supraglottic laryngectomy and irradiation in stage I, II, and III squamous cell carcinoma of the supraglottic larynx. Arch Otolaryngol Head Neck Surg (2007) 133:1044-50. doi:10.1001/archotol.133. 10.1044

7. Bussu F, Almadori G, De Corso E, Rizzo D, Rigante M, Parrilla C, et al. Endoscopic horizontal partial laryngectomy by CO2 laser in the management of supraglottic squamous cell carcinoma. Head Neck (2009) 31:1196-206. doi:10.1002/hed.21085

8. Peretti G, Piazza C, Ansarin M, De Benedetto L, Cocco D, Cattaneo A, et al. Transoral CO2 laser microsurgery for Tis-T3 supraglottic squamous cell carcinomas. Eur Arch Otorhinolaryngol (2010) 267:1735-42. doi:10.1007/ s00405-010-1284-1

9. González-Márquez R, Rodrigo JP, Llorente JL, Alvarez-Marcos C, Díaz JP, Suárez C. Transoral CO2 laser surgery for supraglottic cancer. Eur Arch Otorhinolaryngol (2012) 269:2081-6. doi:10.1007/s00405-012-2016-5

10. Canis M, Martin A, Ihler F, Wolff HA, Kron M, Matthias C, et al. Results of transoral laser microsurgery for supraglottic carcinoma in 277 patients. Eur Arch Otorhinolaryngol (2013) 270:2315-26. doi:10.1007/s00405-012-2327-6

11. Canis M, Ihler F, Martin A, Wolff HA, Matthias C, Steiner W. Results of 226 patients with T3 laryngeal carcinoma after treatment with transoral laser microsurgery. Head Neck (2014) 36:652-9. doi:10.1002/hed.23338

12. Pantazis D, Liapi G, Kostarelos D, Kyriazis G, Pantazis TL, Riga M. Glottic and supraglottic pT3 squamous cell carcinoma: outcomes with transoral laser microsurgery. Eur Arch Otorhinolaryngol (2015) 272:1983-90. doi:10.1007/ s00405-015-3611-z

13. Vilaseca I, Bernal-Sprekelsen M, Blanch L. Transoral laser microsurgery for T3 laryngeal tumors: prognostic factors. Head Neck (2010) 32:929-38. doi:10.1002/hed.21288

14. Vilaseca I, Blanch JL, Berenguer J, Grau JJ, Verger E, Muxí Á, et al. Transoral laser microsurgery for locally advanced (T3-T4a) supraglottic squamous cell carcinoma: sixteen years of experience. Head Neck (2016) 38:1050-7. doi:10.1002/hed.24408

15. Mannelli G, Lazio MS, Luparello P, Gallo O. Conservative treatment for advanced T3-T4 laryngeal cancer: meta-analysis of key oncological outcomes. Eur Arch Otorhinolaryngol (2018) 275:27-38. doi:10.1007/s00405017-4799-X

16. Forastiere AA, Goepfert H, Maor M, Pajak TF, Weber R, Morrison W, et al. Concurrent chemotherapy and radiotherapy for organ preservation in

\section{AUTHOR CONTRIBUTIONS}

PA contributed to the conception of the work, data analysis and interpretation, manuscript writing, and final approval of the version to be published. MG-D contributed to the data collection and analysis and drafting the manuscript. AF contributed to the data collection and analysis and drafting the manuscript. CS contributed to the data analysis. JH contributed to the statistical calculations.

advanced laryngeal cancer. N Engl J Med (2003) 349:2091-8. doi:10.1056/ NEJMoa031317

17. Forastiere AA, Zhang Q, Weber RS, Maor MH, Goepfert H, Pajak TF, et al. Long-term results of RTOG 91-11: a comparison of three nonsurgical treatment strategies to preserve the larynx in patients with locally advanced larynx cancer. J Clin Oncol (2013) 31:845-52. doi:10.1200/JCO.2012.43.6097

18. Forastiere AA, Ismaila N, Lewin JS, Nathan CA, Adelstein DJ, Eisbruch A, et al. Use of larynx-preservation strategies in the treatment of laryngeal cancer: American Society of Clinical Oncology clinical practice guideline update. J Clin Oncol Nov (2017) 27:JCO2017757385. doi:10.1200/JCO.2017.75.7385

19. Machtay M, Moughan J, Trotti A, Garden AS, Weber RS, Cooper JS, et al. Factors associated with severe late toxicity after concurrent chemoradiation for locally advanced head and neck cancer: an RTOG analysis. J Clin Oncol (2008) 26:3582-9. doi:10.1200/JCO.2007.14.8841

20. Hoffman HT, Porter K, Karnell JH, Cooper JS, Weber RS, Langer CJ, et al. Laryngeal cancer in the United States: changes in demographics, patterns of care, and survival. Laryngoscope (2006) 116(Suppl 111):1-13. doi:10.1097/01. mlg.0000236095.97947.26

21. Chen AY, Halpern M. Factors predictive of survival in advanced laryngeal cancer. Arch Otolaryngol Head Neck Surg (2007) 133:1270-6. doi:10.1001/ archotol.133.12.1270

22. Garcia Lorenzo J, Montoro Martinez V, Rigo Quera A, Codina Aroca A, Lopez Vilas M, Quer Agusti M, et al. Modifications in the treatment of advanced laryngeal cancer throughout the last 30 years. Eur Arch Otorhinolaryngol (2017) 274:3449-55. doi:10.1007/s00405-017-4639-z

23. Steiner W, Ambrosch P. Endoscopic Laser Surgery of the Upper Aerodigestive Tract. Stuttgart, New York: Thieme (2000).

24. Remacle M, Hantzakos A, Eckel H, Evrard AS, Bradley PJ, Chevalier D, et al. Endoscopic supraglottic laryngectomy: a proposal for a classification by the working committee on nomenclature, European Laryngological Society. Eur Arch Otorhinolaryngol (2009) 266:993-8. doi:10.1007/s00405-008-0901-8

25. Chen AY, Frankowski R, Bishop-Leone J, Hebert T, Leyk S, Lewin J, et al. The development and validation of a dysphagia-specific quality-of-life questionnaire for patients with head and neck cancer. Arch Otolaryngol Head Neck Surg (2001) 127:870-6.

26. Jacobson BH, Johnson A, Grywalski C, Silbergleit A, Jacobson G, Benninger MS, et al. The voice handicap index (VHI): development and validation. Am J Speech Lang Pathol (1997) 6:66-70. doi:10.1044/1058-0360.0603.66

27. List MA, Ritter-Sterr C, Lansky SB. A performance status scale for head and neck cancer patients. Cancer (1990) 66:564-9. doi:10.1002/10970142(19900801)66:3<564::AID-CNCR2820660326>3.0.CO;2-D

28. List MA, D’Antonio LL, Cella DF, Siston A, Mumby P, Haraf D, et al. The performance status scale for head and neck cancer patients and the functional assessment of cancer therapy-head and neck scale: a study of utility and validity. Cancer (1996) 77:2294-301. doi:10.1002/(SICI)1097-0142(19960601) 77:11<2294::AID-CNCR17>3.0.CO;2-S

29. Bernier J, Domenge C, Ozsahin M, Matuszewska K, Lefebvre JL, Greiner RH, et al. Postoperative irradiation with or without concomitant chemotherapy for locally advanced head and neck cancer. N Engl J Med (2004) 350: 1945-1052. doi:10.1056/NEJMoa032641

30. Cooper JS, Pajak TF, Forastiere AA, Jacobs J, Campbell BH, Saxman SB, et al. Postoperative concurrent radiotherapy and chemotherapy for highrisk squamous cell carcinoma of the head and neck. N Engl J Med (2004) 350:1937-44. doi:10.1056/NEJMoa032646

31. Cooper JS, Zhang Q, Pajak TF, Forastiere AA, Jacobs J, Saxman SB, et al. Longterm follow-up of the RTOG 9501/Intergroup phase III trial: postoperative 
concurrent radiation therapy and chemotherapy in high-risk squamous cell carcinoma of the head \& neck. Int J Radiat Oncol Biol Phys (2012) 84:1198-205. doi:10.1016/j.ijrobp.2012.05.008

32. Roh JL, Kim DH, Park CI. Voice, swallowing and quality of life in patients after transoral laser surgery for supraglottic carcinomas. J Surg Oncol (2008) 98:184-9. doi:10.1002/jso.21101

33. Karatzanis AD, Psychogios G, Zenk J, Waldfahrer F, Hornung J, Velegrakis GA, et al. Evaluation of available surgical management options for early supraglottic cancer. Head Neck (2010) 32:1048-55. doi:10.1002/hed.21289

34. Ambrosch P, Rödel R, Kron M, Steiner W. Die transorale Lasermikrochirurgie des Larynxkarzinoms. Eine retrospektive Analyse von 657 Patientenverläufen. Onkologe (2001) 7:505-12. doi:10.1007/s007610170103

35. Prades JM, Simon PG, Timoshenko AP, Dumollard JM, Schmitt T, Martin C. Extended and standard supraglottic laryngectomies: a review of 110 patients. Eur Arch Otorhinolaryngol (2005) 262:947-52. doi:10.1007/s00405-004-0882-1

36. Bron LP, Soldati D, Monod ML, Megevand C, Brossard E, Monnier P, et al. Horizontal partial laryngectomy for supraglottic squamous cell carcinoma. Eur Arch Otorhinolaryngol (2005) 262:302-6. doi:10.1007/s00405004-0824-y

37. Sevilla MA, Rodrigo JP, Llorente JL, Cabanillas R, Lopez F, Suarez C. Supraglottic laryngectomy: analysis of 267 cases. Eur Arch Otorhinolaryngol (2008) 265:11-6. doi:10.1007/s00405-007-0415-9

38. Scola B, Fernandez-Vega M, Martinez T, Fernandez-Vega S, Ramirez C. Management of cancer of the supraglottis. Otorhinolaryngol Head Neck Surg (2001) 124:195-8. doi:10.1067/mhn.2001.112202

39. Herranz J, Martinez-Vidal J, Martinez Moran A. Supraglottic laryngectomy. Still on-going. Acta Otorrinolaryngol Esp (2006) 57:235-41. doi:10.1016/ S0001-6519(06)78699-X
40. Schwaab G, Kolb F, Julieron M, Janot F, Le Ridant AM, Mamelle G, et al. Subtotal laryngectomy with cricohyoidopexy as first treatment procedure for supraglottic carcinoma: Institut Gustave-Roussy experience (146 cases, 1974-1997). Eur Arch Otorhinolaryngol (2001) 258:246-9. doi:10.1007/ s004050100348

41. Piazza C, Barbieri D, Del Bon F, Grazioli P, Perotti P, Paderno A, et al. Functional outcomes after different types of transoral supraglottic laryngectomy. Laryngoscope (2015) 126:1131-5. doi:10.1002/lary.25562

42. Ambrosch P, Fazel A. Functional organ preservation in laryngeal and hypopharyngeal cancer. GMS Curr Top Otorhinolaryngol Head Neck Surg (2011) 10:Doc02. doi:10.3205/cto000075

43. Butler A, Rigby MH, Scott J, Trites J, Hart R, Taylor SM. A retrospective review in the management of T3 laryngeal squamous cell carcinoma: an expanding indication for transoral laser microsurgery. J Otolaryngol Head Neck Surg (2016) 45:34-9. doi:10.1186/s40463-016-0147-1

Conflict of Interest Statement: The authors declare that the research was conducted in the absence of any commercial or financial relationships that could be construed as a potential conflict of interest.

Copyright (c) 2018 Ambrosch, Gonzalez-Donate, Fazel, Schmalz and Hedderich. This is an open-access article distributed under the terms of the Creative Commons Attribution License (CC BY). The use, distribution or reproduction in other forums is permitted, provided the original author(s) and the copyright owner are credited and that the original publication in this journal is cited, in accordance with accepted academic practice. No use, distribution or reproduction is permitted which does not comply with these terms. 


\section{OPEN ACCESS}

Edited by:

David I. Rosenthal,

University of Texas MD Anderson

Cancer Center, United States

Reviewed by: Torahiko Nakashima, Kyushu University, Japan Wojciech Golusiński,

Department of Head and Neck Surgery, Poland

*Correspondence: Cesare Piazza ceceplaza@libero.it, cesare.piazza@istitutotumori.mi.it

Specialty section:

This article was submitted to Head and Neck Cancer, a section of the journal Frontiers in Oncology

Received: 13 July 2017 Accepted: 07 November 2017 Published: 29 November 2017

Citation:

Mora F, Missale F, Incandela F, Filauro M, Parrinello G, Paderno A, Della Casa P, Piazza $C$ and Peretti $G$ (2017) High Frequency Jet Ventilation during Transoral Laser Microsurgery for Tis-T2 Laryngeal Cancer.

Front. Oncol. 7:282. doi: 10.3389/fonc.2017.00282

\section{High Frequency Jet Ventilation during Transoral Laser Microsurgery for Tis-T2 Laryngeal Cancer}

Francesco Mora ${ }^{1}$, Francesco Missale ${ }^{1}$, Fabiola Incandela ${ }^{1}$, Marta Filauro ${ }^{1}$, Giampiero Parrinello', Alberto Paderno², Palmiro Della Casa ${ }^{3}$, Cesare Piazza ${ }^{4 *}$ and Giorgio Peretti ${ }^{1}$

${ }^{1}$ Department of Otorhinolaryngology - Head and Neck Surgery, University of Genoa, Genoa, Italy, ${ }^{2}$ Department of Otorhinolaryngology - Head and Neck Surgery, University of Brescia, Brescia, Italy, ${ }^{3}$ Department of Anaesthesiology, Ospedale Policlinico San Martino, Genoa, Italy, ${ }^{4}$ Department of Otorhinolaryngology - Head and Neck Surgery, Fondazione IRCCS, National Cancer Institute of Milan, University of Milan, Milan, Italy

Background: Transoral laser microsurgery (TLM) for early to intermediate laryngeal squamous cell cancer (SCC) can be technically challenging when adequate exposure of the posterior laryngeal compartment is required due to the presence of the orotracheal tube. The goal of our study was to analyze the efficacy of high frequency jet ventilation (HFJV) in achieving appropriate laryngeal exposure and safe oncologic resection of lesions located in such a position.

Methods: We reviewed the clinical records of 62 patients affected by Tis-T2 SCC of the posterior laryngeal compartment treated by TLM between 02/2012 and 12/2016. The cohort was divided into two groups according to the anesthesiologic technique used: Group A included patients treated using intraoperative infraglottic HFJV, while Group B encompassed patients treated by standard orotracheal intubation. The main outcome was postoperative surgical margin status. Group comparison analysis was performed.

Results: Significant difference in deep margin status was observed between the two groups: in Group A, the rate of negative deep margins was $86 \%$ compared to $56 \%$ in Group B $(p=0.04)$. A trend of better overall and superficial margin control was observed for patients treated using HFJV (Group A), although no statistical significance was achieved.

Conclusion: Use of HFJV during TLM allows easier and safer management of patients affected by Tis-T2 SCC of the posterior laryngeal compartment, reducing the rates of positive superficial and deep surgical margins.

Keywords: high frequency jet ventilation, transoral laser microsurgery, laryngeal cancer, glottic cancer, supraglottic cancer, surgical margins, carbon dioxide laser, narrow band imaging

\section{INTRODUCTION}

Transoral laser microsurgery (TLM), mostly applied in early-intermediate categories laryngeal squamous cell carcinomas (SCC), is characterized by an "ultra-narrow margins" approach requiring paramount target exposure and precise visualization of the superficial tumor extent, which together make the process of surgical resection within $1 \mathrm{~mm}$ clear margins oncologically safe

Abbreviations: HFJV, high frequency jet ventilation; TLM, transoral laser microsurgery; NBI, narrow band imaging; WL, white light; SCC, squamous cell carcinoma. 
but, at the same time, technically challenging $(1,2)$. Obtaining adequate laryngeal exposure is one of the main issues crucially influencing the successful feasibility of TLM and its preoperative objective assessment should be always considered as an essential prerequisite $(3,4)$. Orotracheal intubation is considered the routine standard of care for carbon dioxide laser procedures under general anesthesia, even though the tube located at the level of the interarytenoid area may blind the posterior third of both vocal cords, the medial surface of the arytenoids, and the posterior commissure. Hence, treatment of lesions involving such areas is consequently penalized by suboptimal visualization, with risk of incomplete resection and a higher rate of positive superficial margins even when an experienced surgeon tries to work by displacing the tube toward the anterior commissure, intercalating dedicated laryngoscopes like the Karlan-Ossoff (Pilling, Philadelphia, PA, USA) between the tube itself and the posterior commissure. In fact, due to the very nature of the orotracheal intubation, this invariably causes difficulties in properly working at the level of the posterior part of the glottic and supraglottic subsites. Moreover, the altered line of sight that this maneuver carries working through the microscope may cause a higher risk of laser refraction within the laryngoscope. One possible alternative to such a tricky scenario is through creation of a temporary tracheotomy which, by completely removing the issue of orotracheal intubation, clearly allows gaining more space to manage the lesion in the posterior aspect of the larynx, albeit at the price of extra-morbidity for the patient (5).

In recent decades, new endoscopic tools, laryngoscopes, suspension systems, and anesthesiologic equipment have been developed to increase the diagnostic accuracy during TLM and optimize exposure of the related surgical field. In particular, infraglottic high frequency jet ventilation (HFJV) is a well-known anesthesiologic technique based on gas puffs delivered under high pressure through a small catheter placed into the airway, thus creating an open ventilation system. The insufflation of gas through the jet nozzle is an active process, whereas exhalation occurs in a passive way (6). HFJV is useful in all situations where access to the airway is potentially hindered by the orotracheal tube, thanks to the small size of the catheter (6-8).

The aim of this retrospective study was to evaluate, in selected "posterior" Tis-T2 laryngeal cancers of the arytenoid and/or posterior third of the vocal folds, extending to the interarytenoid area up to the posterior commissure, possible advantages of the use of HFJV in terms of reduction of positive surgical margins compared to a control group of patients treated by the same surgeons and surgical techniques using standard orotracheal intubation.
A

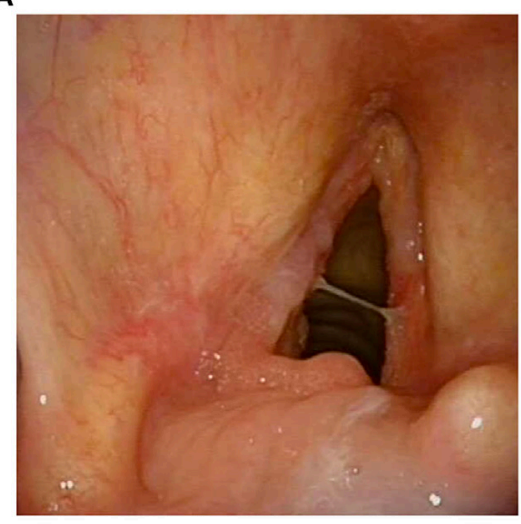

C

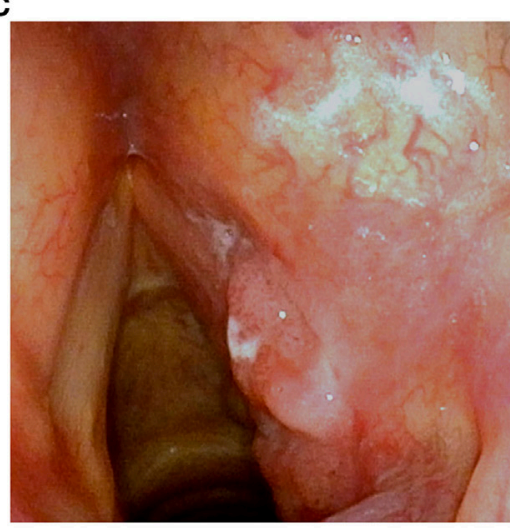

B

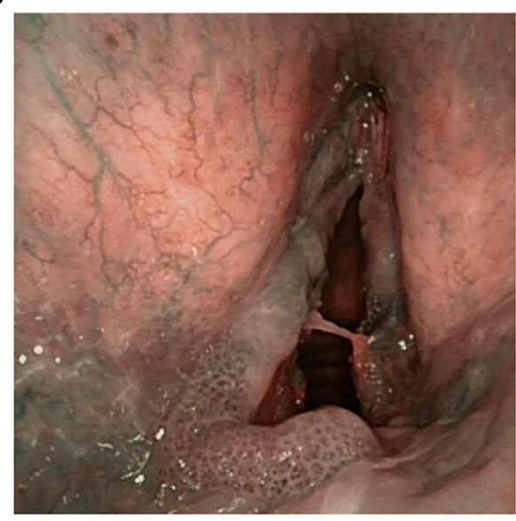

D

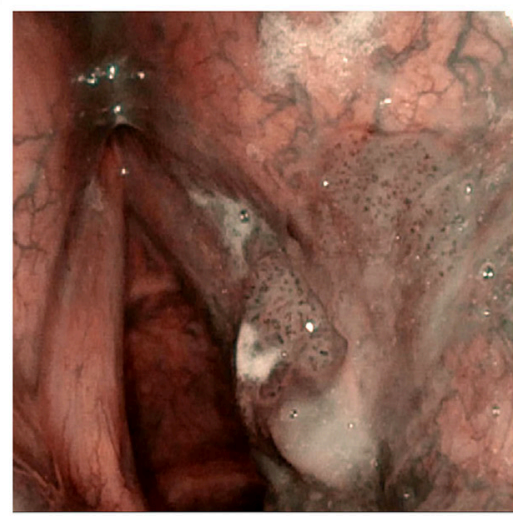

FIGURE 1 | Preoperative videoendoscopy of a SCC of the posterior third of the left vocal cord involving the medial surface of the arytenoid and the posterior commissure in white light (WL) (A) and narrow band imaging (NBI) (B); SCC of the posterior third of the right vocal cord involving the ventricular band and the medial aspect of the arytenoid in WL (C) and NBI (D). 


\section{MATERIALS AND METHODS}

Sixty-two patients, affected by selected Tis-T2 SCC involving the posterior third of the vocal fold and/or the medial aspect of the arytenoid with adjacent interarytenoid area (Figures 1A-D), were treated by TLM from February 2012 to December 2016 at the Department of Otorhinolaryngology-Head and Neck Surgery of the University of Genoa, Italy, and included in the present analysis. Ethical review and approval was not required for this study in accordance with the national and institutional requirements. However, every patient preoperatively signed a consent form for disclosure of privacy in managing personal data for scientific purposes.

All of them had been preoperatively assessed in the office by the Laryngoscore, in order to rule out impossible or difficult laryngeal exposure (frequently encountered for a Laryngoscore's value > 9) (3). Preoperative assessment was performed in all patients by flexible transnasal videoendoscope under white light (WL) and narrow band imaging Olympus Medical System Corporation, Tokyo, Japan, and integrated in the intraoperative setting by a multiprospective view obtained with rigid angled telescopes $\left(0^{\circ}, 30^{\circ}\right.$, and $\left.70^{\circ}\right)$ to better visualize the superficial extension and surgical margins of the lesion by recognizing the atypical vascular patterns inside the lesion and surrounding tissues. Surgical procedures were performed under microlaryngoscopy with carbon dioxide laser (Lumenis Encore Ultrapulse, Tel
Aviv, Israel) combined with an Acublade micromanipulator using different laryngoscopes to obtain adequate laryngeal exposure. Patients with anesthesiologic HFJV exclusion criteria such as morbid obesity, stiff thorax, and restrictive and/or obstructive pulmonary diseases were excluded from the study (6).

The entire cohort was divided in two groups based on the anesthesiologic technique used. Group A encompassed 14 patients (12 males, 2 females, ranging in age from 34 to 82 years, median 75.5 ) treated from June 2013 to December 2016 using intraoperative HFJV: 11 (79\%) of them were mostly glottic tumors with cranial extension to the arytenoid, while 3 (21\%) originated mainly from the arytenoid and presented marginal caudal involvement of the posterior third of the vocal fold and/or posterior commissure at the glottic level. Group B encompassed 48 patients (44 males, 4 females, ranging in age from 40 to 86 years, median 65.5 ) treated from February 2012 to May 2013 using standard orotracheal intubation: again, distribution between mainly glottic (43 patients, or $90 \%$ ) vs. supraglottic lesions (5 cases, or $10 \%$ ) is reported in Table 1.

High frequency jet ventilation (Monsoon III, Acutronic, Switzerland) was performed using a transglottic double lumen laser catheter made of incombustible tetrafluoroethylene, with an outer diameter of $4 \mathrm{~mm}$. The typical working setting of HFJV in the present series was between 140 and 155 cycles per minute, with a drive pressure from 1.5 to 2.5 bar. As usual in every laser procedure, the $\mathrm{PO}_{2}$ must not exceed 30\%: this is checked by

TABLE 1 | Patient characteristics, tumor category, grading, margin status of Group A and B.

\begin{tabular}{|c|c|c|c|c|c|}
\hline Variables & & Overall series, $n(\%)$ & $\begin{array}{c}\text { Group A (high frequency } \\
\text { jet ventilation), } \boldsymbol{n}(\%)\end{array}$ & $\begin{array}{l}\text { Group B (standard } \\
\text { intubation), } n(\%)\end{array}$ & $p$ Value \\
\hline Age & Median & 67.5 & 75.5 & 65.5 & $0.004^{\#}$ \\
\hline \multirow[t]{3}{*}{ Gender } & & & & & $0.51^{\star}$ \\
\hline & Male & $56(90)$ & $12(86)$ & $44(92)$ & \\
\hline & Female & $6(10)$ & $2(14)$ & $4(8)$ & \\
\hline \multirow[t]{3}{*}{ Previous treatment } & & & & & $0.43^{\star}$ \\
\hline & No & $45(73)$ & $9(64)$ & $36(75)$ & \\
\hline & Yes & $17(27)$ & $5(36)$ & $12(25)$ & \\
\hline \multirow[t]{3}{*}{ Site } & & & & & $0.28^{\star}$ \\
\hline & Glottis & $54(87)$ & $11(79)$ & $43(90)$ & \\
\hline & Supraglottis & $8(13)$ & $3(21)$ & $5(10)$ & \\
\hline \multirow[t]{3}{*}{ Tumor category } & & & & & $0.34^{*}$ \\
\hline & Tis-T1 & $46(74)$ & $9(64)$ & $37(77)$ & \\
\hline & $\mathrm{T} 2$ & $16(26)$ & $5(36)$ & $11(23)$ & \\
\hline \multirow[t]{3}{*}{ Grading } & & & & & $0.87^{*}$ \\
\hline & G1 & $21(34)$ & $5(36)$ & $16(33)$ & \\
\hline & G2-3 & $41(66)$ & $9(64)$ & $32(67)$ & \\
\hline \multirow[t]{3}{*}{ Margins } & & & & & $0.10^{\star}$ \\
\hline & Negative & $24(39)$ & $8(57)$ & $16(33)$ & \\
\hline & Positive/close & $38(61)$ & $6(43)$ & $32(67)$ & \\
\hline \multirow[t]{3}{*}{ Deep margins } & & & & & $0.04^{*}$ \\
\hline & Negative & $39(63)$ & $12(86)$ & $27(56)$ & \\
\hline & Positive/close & $23(37)$ & $2(14)$ & $21(44)$ & \\
\hline \multirow[t]{3}{*}{ Superficial margins } & & & & & $0.64^{*}$ \\
\hline & Negative & $32(52)$ & $8(57)$ & $24(50)$ & \\
\hline & Positive/close & $30(48)$ & $6(43)$ & $24(50)$ & \\
\hline \multirow[t]{3}{*}{ Posterior/inferior margins } & & & & & $0.33^{\star}$ \\
\hline & Negative & $42(68)$ & $11(79)$ & $31(65)$ & \\
\hline & Positive/close & $20(32)$ & $3(21)$ & $17(35)$ & \\
\hline
\end{tabular}

*p value estimated by chi-square test.

"p value estimated by the Mann-Whitney test. 


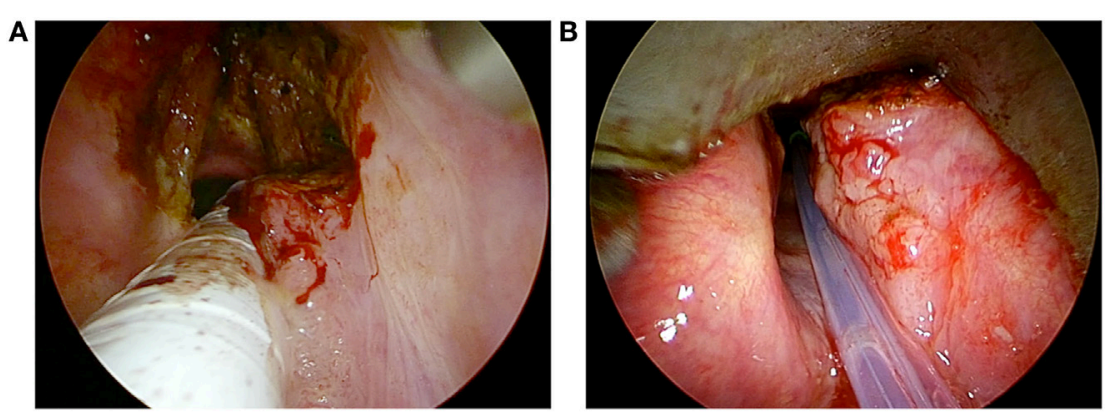

FIGURE 2 | Intraoperative endoscopy of a T2 glottic SCC involving both vocal cords and the right arytenoid treated by transoral laser microsurgery with orotracheal intubation (A), switching to high frequency jet ventilation to manage the residual part of the lesion at the level of the posterior laryngeal compartment (B).

the machine itself, alarming the surgeon when the $\mathrm{PO}_{2}$ reaches potentially dangerous levels.

In some cases, in order to avoid prolonged use of HFJV during the procedure, the surgeon switched from the standard orotracheal intubation (used at the beginning of surgery to manage the anterior and mid portions of the lesion) to HFJV only for treating the posterior part of the larynx (Figures 2A,B).

We defined surgical margins as negative if invasive cancer/ carcinoma in situ/severe dysplasia was more than $1 \mathrm{~mm}$ from the resection margin, and close if at less than $1 \mathrm{~mm}$.

Categorical data with absolute and relative frequencies were described, and comparisons between the two groups were performed using a chi-square test. Comparison between continuous variables was done by Mann-Whitney test. Two-sided $p$ values $<0.05$ were considered statistically significant. The parameters evaluated were gender, age, previous treatment, site (glottis vs. supraglottis), pT category (pTis-pT1 vs. pT2), histological grade (G1 vs. G2-G3), surgical margins (negative vs. close-positive), superficial margins (negative vs. close-positive), posterior and inferior margins (negative vs. close-positive), and deep margins (negative vs. close-positive). Statistical analysis was performed using GraphPad Prism software, Version 6.0 (San Diego, CA, USA).

\section{RESULTS}

No statistically significant difference was found between the two groups regarding gender, previous treatment, glottis vs. supraglottic sites, pT category, and grading. Age was significantly higher in Group A $(p=0.004)$. A significant difference in deep margin status was observed between the two groups: in Group A, the rate of negative deep margins was $86 \%$ compared to $56 \%$ in Group B $(p=0.04)$. No statistically significant difference was found in Group A vs. Group B between the overall margin status (57\% of safe margins vs. $33 \%$ ), that of superficial margins (57 vs. $50 \%$ ), and posterior/inferior margins (79 vs. $65 \%$ ), with only a trend for better margin control in patients treated by HFJV (Table 1).

\section{DISCUSSION}

Early glottic SCC uncommonly arises or involves the vocal process, the medial aspect of the arytenoid and/or posterior commissure, with the vast majority located at the level of the middle third of the vocal fold, and potentially spreading toward the anterior commissure or through the ventricle at the level of the false vocal folds or epiglottic petiole (9). Even early supraglottic SCC primarily arising from the arytenoid are quite rarely encountered. In the series by Laccourreye et al., 21 patients with T1 lesions arising from the arytenoid were reported: of 10 patients treated with free resection margins, only 6 received TLM, while 4 were managed by open neck approaches (9). In both posteriorly located glottic and supraglottic cancers, if surgery by TLM is planned, complete visualization of the entire surgical field is mandatory to obtain an accurate resection within oncologically safe margins and, consequently, achieve local control by unimodal treatment.

The ability to deliver HFJV permits ventilating the patient through a narrow catheter that allows achieving complete exposure of the posterior third of the larynx due to its small diameter and interarytenoid positioning, thus reducing the need for orotracheal tube anterior dislodgement or tracheotomy. With such a technique, the surgeon has a wider space for surgical maneuvers, even in a traditionally difficult clinical scenario such as that represented by posterior glottic/supraglottic tumors (10).

The two groups of patients in the present series were homogeneous considering T category $(p=0.34)$ and other clinicopathological variables. Our data confirmed that HFJV in selected cases favors a significantly greater deep margin control, with reduction of the positive margins rate compared to standard orotracheal intubation ( 14 vs. $44 \%$; $p=0.04$ ). Moreover, a trend toward better overall and superficial margins control in Group A was also observed, even though the difference did not reach statistical significance.

As with every surgical and anesthesiologic technique, HFJV also has specific indications, contraindications, and possible drawbacks. The most common complications are hypoxia, hypercarbia and, rarely, emphysema and blood aspiration $(8,11)$. In fact, HFJV creates an open system in which blood aspiration may potentially represent an issue, even though the expiration air flow across the glottis usually minimizes such a complication which, in the present series, was never encountered.

To reduce the risk of hypoxia or hypercarbia during TLM procedures lasting more than $1 \mathrm{~h}$, it is possible to start the resection from the anterior commissure with the orotracheal tube 
in situ, later switching to HFJV for resection of the posterior part of the lesion $(6,12)$. This trick also allows managing bulky tumors with posterior extension by HFJV; in fact, these should be always considered a contraindication to the use of HFJV due to the potential limitation of back expiratory airflow, with ensuing risk of lung barotraumas and pneumothorax. In fact, raising the drive pressure and lowering the number of cycles per minute are the only means that anesthesiologists have to reduce $\mathrm{PCO}_{2}(6,13)$. However, in addition to the risk of barotrauma and aspiration, this may cause fluttering of vocal cords that makes TLM potentially less precise and cumbersome (6). In our experience, the optimal compromise between such requirements was found for a frequency around 140 cycles per minute with a drive pressure of 1.5 bar. Moreover, with such parameters, the intraoperative vibration of the distal tip of the catheter and vocal folds is reduced to a minimum and usually does not interfere with the required surgical accuracy.

\section{REFERENCES}

1. Peretti G, Piazza C, Cocco D, De Benedetto L, Del Bon F, Redaelli De Zinis LO, et al. Transoral $\mathrm{CO}_{2}$ laser treatment for Tis-T3 glottic cancer: the University of Brescia experience on 595 patients. Head Neck (2010) 32:977-83. doi:10.1002/ hed.21278

2. Sjögren EV. Transoral laser microsurgery in early glottic lesions. Curr Otorhinolaryngol Rep (2017) 5:56-68. doi:10.1007/s40136-0170148-2

3. Piazza C, Mangili S, Bon FD, Paderno A, Grazioli P, Barbieri D, et al. Preoperative clinical predictors of difficult laryngeal exposure for microlaryngoscopy: the Laryngoscore. Laryngoscope (2014) 124:2561-7. doi:10.1002/ lary. 24803

4. Peretti G, Piazza C, Mora F, Garofolo S, Guastini L. Reasonable limits for transoral laser microsurgery in laryngeal cancer. Curr Opin Otolaryngol Head Neck Surg (2016) 24:135-9. doi:10.1097/MOO.0000000000000240

5. De Virgilio A, Park YM, Kim WS, Baek SJ, Kim SH. How to optimize laryngeal and hypopharyngeal exposure in transoral robotic surgery. Auris Nasus Larynx (2013) 40:312-9. doi:10.1016/j.anl.2012.07.017

6. Biro P. Jet ventilation for surgical interventions in the upper airway. Anesthesiol Clin (2010) 28:397-409. doi:10.1016/j.anclin.2010.07.001

7. Barakate M, Maver E, Wotherspoon G, Havas T. Anaesthesia for microlaryngeal and laser laryngeal surgery: impact of subglottic jet ventilation. J Laryngol Otol (2010) 124:641-5. doi:10.1017/S0022215109992532

8. Hu A, Weissbrod PA, Maronian NC, Hsia J, Davies JM, Sivarajan GK, et al. Hunsaker Mon-Jet tube ventilation: a 15-year experience. Laryngoscope (2012) 122:2234-9. doi:10.1002/lary.23491

\section{CONCLUSION}

Use of HFJV during TLM allows easier and safer management of patients affected by Tis-T2 SCC involving the medial aspect of the arytenoid and posterior part of the glottis. Compared with standard orotracheal intubation or tracheotomy, HFJV allows reduction of perioperative morbidity and rates of positive superficial and deep margins.

\section{AUTHOR CONTRIBUTIONS}

FMO: study design, preparation of manuscript, and revision of manuscript. FMI: study design, data collection, data analysis, and preparation of manuscript. FI, MF, and GPA: data collection and preparation of manuscript. AP and PC: study design and preparation of manuscript; CP and GPE: study design and revision of manuscript.

9. Laccourreye O, Weinstein G, Chabardes E, Housset M, Laccourreye H, Brasnu D. T1 squamous cell carcinoma of the arytenoid. Laryngoscope (1992) 102:896-900. doi:10.1288/00005537-199208000-00009

10. Orloff LA, Parhizkar N, Ortiz E. The Hunsaker Mon-Jet ventilation tube for microlaryngeal surgery: optimal laryngeal exposure. Ear Nose Throat J (2002) 81:390-4.

11. Cook TM, Alexander R. Major complications during anaesthesia for elective laryngeal surgery in the UK: a national survey of the use of high-pressure source ventilation. Br J Anaesth (2008) 101:266-72. doi:10.1093/bja/aen139

12. Cheng J, Woo P. Rescue microlaryngoscopy: a protocol for utilization of four techniques in overcoming challenging exposures in microlaryngeal surgery. J Voice (2012) 26:590-5. doi:10.1016/j.jvoice.2011.10.009

13. Helmstaedter V, Tellkamp R, Majdani O, Warnecke A, Lenarz T, Durisin M. High-frequency jet ventilation for endolaryngotracheal surgery - chart review and procedure analysis from the surgeon's and the anaesthesiologist's point of view. Clin Otolaryngol (2015) 40:341-8. doi:10.1111/coa.12376

Conflict of Interest Statement: The authors declare that the research was conducted in the absence of any commercial or financial relationships that could be construed as a potential conflict of interest.

Copyright (C) 2017 Mora, Missale, Incandela, Filauro, Parrinello, Paderno, Della Casa, Piazza and Peretti. This is an open-access article distributed under the terms of the Creative Commons Attribution License (CC BY). The use, distribution or reproduction in other forums is permitted, provided the original author(s) or licensor are credited and that the original publication in this journal is cited, in accordance with accepted academic practice. No use, distribution or reproduction is permitted which does not comply with these terms. 
OPEN ACCESS

Edited by:

Christian Simon,

Centre Hospitalier Universitaire

Vaudois (CHUV), Switzerland

Reviewed by:

Sandro J. Stoeckli, Kantonsspital St. Gallen,

Switzerland

Orlando Guntinas-Lichius,

Universitätsklinikum Jena,

Germany

*Correspondence:

Cesare Piazza

cesare.piazza@istitutotumori.mi.it,

ceceplaza@libero.it

Specialty section:

This article was submitted to

Head and Neck Cancer,

a section of the journal

Frontiers in Oncology

Received: 12 February 2018 Accepted: 16 April 2018

Published: 30 April 2018

Citation:

Perotti P, Ferrari M, Montalto N, Lancini D, Paderno A, Incandela F, Buffoli B, Rodella LF and Piazza C (2018) Anatomical Cadaver Study of Endolaryngeal Vascularization: Focus on the Glottis, Supraglottis, and Subglottis From the Transoral Microsurgical Point of View.

Front. Oncol. 8:138

doi: 10.3389/fonc.2018.00138

\section{Anatomical Cadaver Study of Endolaryngeal Vascularization: Focus on the Glottis, Supraglottis, and Subglottis From the Transoral Microsurgical Point of View}

\author{
Pietro Perotti ${ }^{1}$, Marco Ferrari ${ }^{2}$, Nausica Montalto², Davide Lancini ${ }^{2}$, Alberto Paderno ${ }^{1,2}$, \\ Fabiola Incandela ${ }^{1,3}$, Barbara Buffoli ${ }^{4}$, Luigi Fabrizio Rodella ${ }^{4}$ and Cesare Piazza ${ }^{1 *}$ \\ 1 Department of Otorhinolaryngology - Head and Neck Surgery, Fondazione IRCCS, National Cancer Institute of Milan, \\ University of Milan, Milan, Italy, ${ }^{2}$ Department of Otorhinolaryngology - Head and Neck Surgery, University of Brescia, \\ Brescia, Italy, ${ }^{3}$ Department of Otorhinolaryngology - Head and Neck Surgery, University of Genoa, Genoa, Italy, ${ }^{4}$ Department \\ of Clinical and Experimental Sciences, Section of Anatomy and Physiopathology, University of Brescia, Brescia, Italy
}

Introduction: Carbon dioxide laser coagulation during transoral laser microsurgery (TLM) for laryngeal cancer allows control of bleeding from vessels smaller than $0.5 \mathrm{~mm}$. Therefore, larger arteries and veins must be carefully managed by clipping and/or monopolar cautery. The aim of this paper is to detail endolaryngeal vascular anatomy and identify areas of possible bleeding during TLM.

Methods: We performed an anatomical study on a series of 11 fresh-frozen human cadavers. After injection of a bicomponent red silicone into the innominate, left common carotid, and left subclavian arteries, 22 hemilarynges were dissected, the course of the supraglottic, glottic, and subglottic vessels were traced after microdissection of the intervening structures, and their size measured at specific landmark points where such vessels are more frequently encountered during TLM.

Results: Three vessels arising from the superior laryngeal artery were identified after its entry point at the level of the thyro-hyoid membrane: (1) the epiglottic artery (EA), documented in $100 \%$ of cases, a common trunk dividing into two main vessels (2) the postero-inferior artery (PIA), present in 100\% of the specimens, running downward and dividing in a posterior (pPIA), and anterior (aPIA) branches (3) the antero-inferior artery (AIA), present in $95 \%$ of our specimens, running downward to the anterior commissure (AC). Two transverse anastomotic networks (TANs) connected the AIA and PIA, both parallel to the vocal muscle, one lateral (present in 100\% of cases), and another medial (91\% of specimens). Finally, a fourth vessel supplying the glottic plane was found to be the endolaryngeal paracommissural branch of the crico-thyroid artery (PCA), arising from the inferior laryngeal artery and emerging just below the $\mathrm{AC}$, through the crico-thyroid membrane (reported in $100 \%$ of the specimens). This vessel anastomosed in $91 \%$ of cases with the AIA, through one or both of the TANs. 
Conclusion: The course of the endolaryngeal arteries, their relationships with adjacent structures, and size at specific landmark points have been herein described in order to provide surgeons with a map to guide them during the steep learning curve of transoral surgery of the larynx, with special emphasis given to TLM.

Keywords: larynx, anatomy, vascularization, transoral laser microsurgery, bleeding

\section{INTRODUCTION}

Transoral laser microsurgery (TLM) has been established as an effective option in the management of Tis-T2 and selected T3 squamous cell carcinoma (SCC) of the larynx as it allows curative resections of early-intermediate supraglottic and glottic tumors within healthy narrow-free margins, sparing uninvolved portions of the larynx and their associated sphincteric functions. However, to be reproducible and effective, TLM requires specific and long-lasting training, with a perceived steep learning curve, which exposes the patients to a variable (but potentially high) rate of complications, paralleling the increasing stage and extent of disease $(1,2)$. In particular, intraoperative bleeding seems to be especially difficult to predict, while early posttreatment hemorrhage represents a potentially catastrophic complication since the typical postoperative scenario is, in the vast majority of patients, without tracheostomy. Such major postoperative hemorrhagic events are variably reported in the literature to range between 0.6 and $8 \%$ according to the different $\mathrm{T}$ categories and subsites approached (1, 3-5).

The aim of this study was, therefore, to detail the normal anatomy of endolaryngeal vascularization in order to underline the areas at higher risk for potential intra- and postoperative bleedings, with special emphasis given to the technical characteristics of the carbon dioxide laser, which is able to coagulate vessels with a maximum diameter of $0.5 \mathrm{~mm}$. However, the present anatomical data may be applicable not only to TLM but also to other emerging transoral robotic or endoscopic approaches.

\section{MATERIALS AND METHODS}

Between November and December 2016, 11 larynges were harvested at the Section of Anatomy and Physiopathology, University of Brescia, Italy from fresh-frozen cadavers (all Caucasians; 6 males, 5 females; mean age at death, 64 years; range, 51-78) provided by Medcure ${ }^{\circledR}$, USA, after arterial injection of a bicomponent red silicone into the innominate, left common carotid, and left subclavian arteries. Twenty-two hemilarynges were microdissected to analyze their endolaryngeal vascularization: the superior laryngeal artery (SLA) was identified and accurately followed from its entry point at the level of the thyro-hyoid membrane to its smallest branches, by considering all the intervening anastomotic networks. The inferior laryngeal artery (ILA) was analyzed in a similar fashion.

Moreover, we measured the diameter of the main laryngeal vessels using a T444.1XLR-1 Outsider Micrometer caliper (Starrett, Athol, MA, USA) to obtain a tentative measure of their suitability for coagulation by carbon dioxide laser or other surgical tools (clips and/or monopolar cautery). These measurements were obtained at specific landmark points: (1) the epiglottic artery
(EA) was measured at its emergence from the SLA (landmark ea1) and at the level of the lateral margin of the suprahyoid epiglottis where the pharyngo-epiglottic fold joins the cartilage (ea2); (2) the postero-inferior artery (PIA) was measured at its origin; (3) the posterior branch of PIA (pPIA) was measured lateral to the arytenoid body, within the thyro-crico-arytenoid space (ppia1), and within the vocal muscle lateral to the vocal process (ppia2); (4) the antero-inferior artery (AIA) was measured at the level of the false vocal fold (aia1) and glottic plane (aia2).

\section{RESULTS}

In the present study, three vessels arising from the SLA were identified after its entry point through the thyro-hyoid membrane. The EA was found in $100 \%$ of cases. Its caliber ranged from a median of $2.2 \mathrm{~mm}$ at eal to a median of $0.9 \mathrm{~mm}$ at the level of ea2 (Figures 1-3). After emerging from SLA, EA showed a

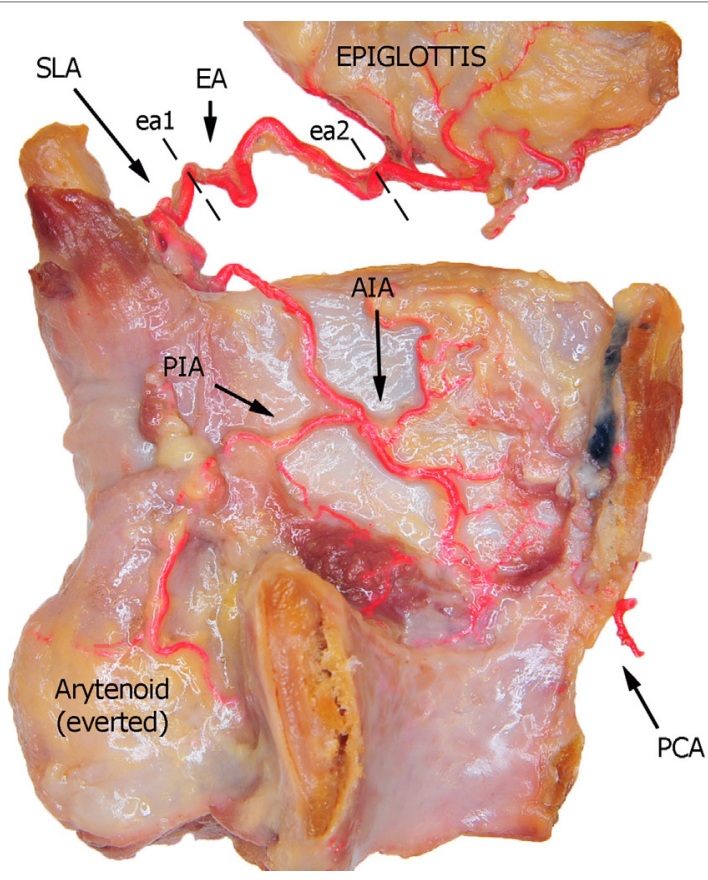

FIGURE 1 | Left fresh-frozen cadaver hemilarynx after midline section of the cricoid and thyroid cartilages, eversion of the ipsilateral arytenoid, and removal of the left aryepiglottic, false, true vocal folds, and paraglottic fat tissue, thus exposing the inner thyroid lamina. Bicomponent red silicone had been injected in the left common and subclavian arteries. Dotted lines ea1 and ea2 represent the landmark points used for measurements of the EA. Abbreviations: SLA, superior laryngeal artery; EA, epiglottic artery; AIA, antero-inferior artery; PIA, postero-inferior artery; PCA, paracommissural branch of the crico-thyroid artery. 
common trunk with a median length of $6 \mathrm{~mm}$ that still divided in two main vessels: (1) the PIA, detected in $100 \%$ of the specimens (with a median diameter of $1.6 \mathrm{~mm}$ at its origin), run downward and

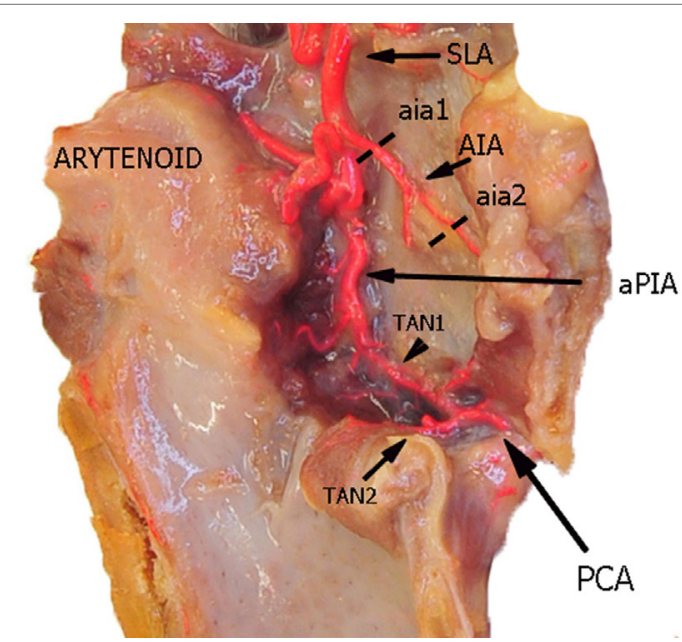

FIGURE 2 | Left fresh-frozen cadaver hemilarynx after midline section of the cricoid and thyroid cartilages. The ipsilateral arytenoid was left in place, while left aryepiglottic, false, true vocal folds, and paraglottic fat tissue had been removed, thus exposing the inner thyroid lamina. Bicomponent red silicone had been injected in the left common carotid and subclavian arteries. Dotted lines aia1 and aia2 are those used for measurements of the AIA.

Abbreviations: SLA, superior laryngeal artery; AIA, antero-inferior artery; aPIA, anterior branch of the postero-inferior artery; PCA, paracommissural branch of the crico-thyroid artery; TAN1, lateral transverse anastomotic network; TAN2, medial transverse anastomotic network. divided again into two further branches, one posterior and lateral to the arytenoid (pPIA) (with a median caliber of $1.4 \mathrm{~mm}$ at ppia) and one directed perpendicularly to the vocal process with an antero-lateral-inferior trajectory and located within the paraglottic space (PGS) and through the vocal muscle (aPIA) (median diameter at apia of $0.9 \mathrm{~mm}$ ) (Figures 1-5); (2) the AIA, detected in $95 \%$ of the specimens, run through the PGS toward the anterior commissure (AC) with an oblique course, and had a median caliber ranging from $1.4 \mathrm{~mm}$ at aia 1 to $0.7 \mathrm{~mm}$ at aia2 (Figures 1, 2, 4 and 5).

Furthermore, two transverse anastomotic networks (TANs) connecting the AIA and PIA, both parallel to the vocal muscle, were found: one lateral (TAN1), which was identified in $100 \%$ of specimens between the muscle and the thyroid cartilage, and one medial (TAN2), which was found in $91 \%$ of specimens, constantly running between the muscle and the conus elasticus, just below the vocal ligament (Figures 2, 4 and 5). TAN1 and TAN2 were extremely variable in size and course from specimen to specimen; briefly, their caliber invariably exceeded $0.5 \mathrm{~mm}$ at the glottic and subglottic levels.

Finally, a fourth vessel supplying the vocal folds was found to be the endolaryngeal branch of the crico-thyroid artery, arising from the ILA and emerging just below the AC through the crico-thyroid membrane, 2-3 mm laterally to the midline. By virtue of its anatomical features, this branch was called paracommissural branch of the crico-thyroid artery (PCA). PCA was measured only outside the crico-thyroid membrane (median diameter of $1.6 \mathrm{~mm}$ ), while it was not precisely sizeable at the level of the endolarynx (but still, a minimum diameter of $0.5 \mathrm{~mm}$ was observed). Identified in $100 \%$ of the specimens, this vessel

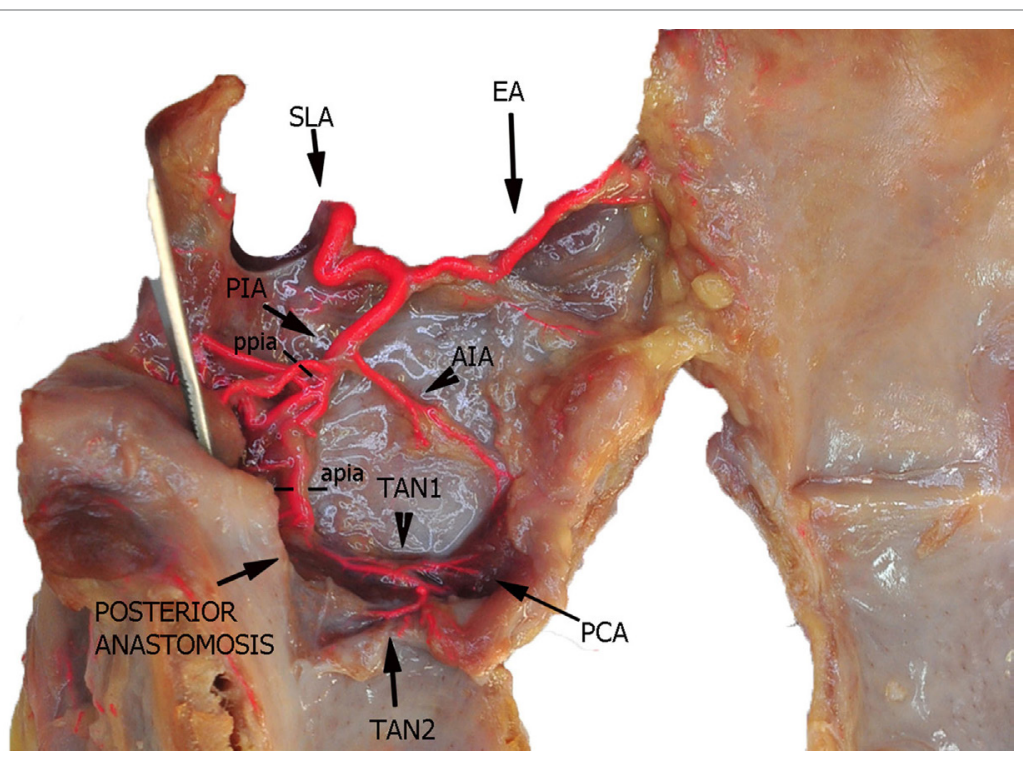

FIGURE 3 | Left fresh-frozen cadaver hemilarynx after midline section of the cricoid and thyroid cartilages and partial separation of the contralateral hemilarynx. The ipsilateral arytenoid, as well as the epiglottis, was left in place and a metallic stick inserted into the piriform sinus, while left aryepiglottic, false, true vocal folds, and paraglottic fat tissue had been removed, thus exposing the inner thyroid lamina. Bicomponent red silicone had been injected in the left common carotid and subclavian arteries. Dotted line ppia was used for measurement of the posterior branch of the PIA, while dotted line apia represents the landmark used for measurement of its anterior branch. Abbreviations: SLA, superior laryngeal artery; AIA, antero-inferior artery; PIA, postero-inferior artery; PCA, paracommissural branch of the crico-thyroid artery; TAN1, lateral transverse anastomotic network; TAN2, medial transverse anastomotic network. 
anastomosed in $91 \%$ of cases with the AIA, via one or both TANs (Figures 1, 2, 4 and 5).

The detection rates and median sizes of the herein described vessels are reported in Table $\mathbf{1 .}$

\section{DISCUSSION}

The first anatomical description of the endolaryngeal vascularization was published by Oki in 1958 (6). After this pioneering study, the topic was revisited by other authors (7-9), even though with an insufficient amount of details for the present purposes, and recently, with a higher resolution, on fixed larynges (10) and by angiography on fresh cadavers (11). To the best of our knowledge,

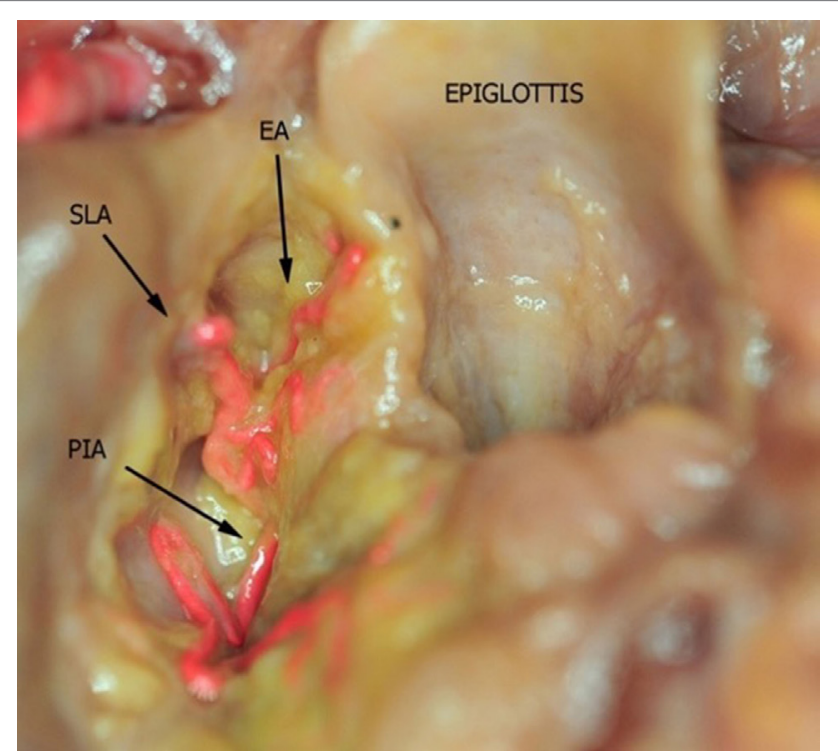

FIGURE 4 | Fresh-frozen cadaver larynx seen from above after partial removal of the fat tissue within the upper and lower paraglottic space. Bicomponent red silicone had been injected in the left common carotid and subclavian arteries. Abbreviations: SLA, superior laryngeal artery; EA, epiglottic artery; PIA, postero-inferior artery. microdissection on fresh-frozen specimens with the specific intention of evaluating its implications during TLM has never been accomplished before.

As it is well known, laryngeal blood supply is guaranteed by both SLA and ILA, which branch inside the organ into a number of smaller vessels whose anatomical description in terms of course, size, and possible variants is scarcely represented in the contemporary head and neck literature and not specifically finalized to TLM. Furthermore, these anatomical details are mostly overlooked even in the most prominent surgical textbooks. However, precise knowledge of the surgical anatomy of endolaryngeal vascularization is, in our opinion, of paramount importance especially when considering potentially dangerous intra- and postoperative bleedings during/after transoral procedures for laryngeal malignancies.

All the specimens of our study presented a classic course of SLA, while the variant described by Iimura et al. (10) and Rusu et al. (12) piercing the thyroid cartilage was not observed. In our experience, SLA always penetrated the thyro-hyoid membrane at a variable distance from the upper edge of the thyroid lamina, running downwards into the upper PGS (at the junction between this visceral compartment and the pre-epiglottic space) and then turning upward giving rise to the EA (also called "ascending branch of SLA" by Imanishi and coworkers) (11). The latter vessel provides blood supply to the ary-epiglottic, pharyngo-epiglottic folds, ipsilateral epiglottis, and glosso-epiglottic valleculae, where it anastomoses with the posterior branches of the lingual artery (13). Proper EA identification and its prophylactic clipping and/or monopolar cauterization are essential during TLM for supraglottic lesions. From a practical point of view, the easiest landmark for such a surgical maneuver is the pharyngo-epiglottic fold (the landmark eal in the present study), even though the presence of the tumor at this level may require more lateral dissection at the level of the entry point of the SLA itself.

After this first division, the common trunk of the SLA (that Imanishi et al. at this point called the "descending branch of SLA") (11) further divides into two main branches: the PIA and AIA. The PIA usually branches in two (P1 and P2 according to the Imanishi nomenclature, aPIA and pPIA in the present study)

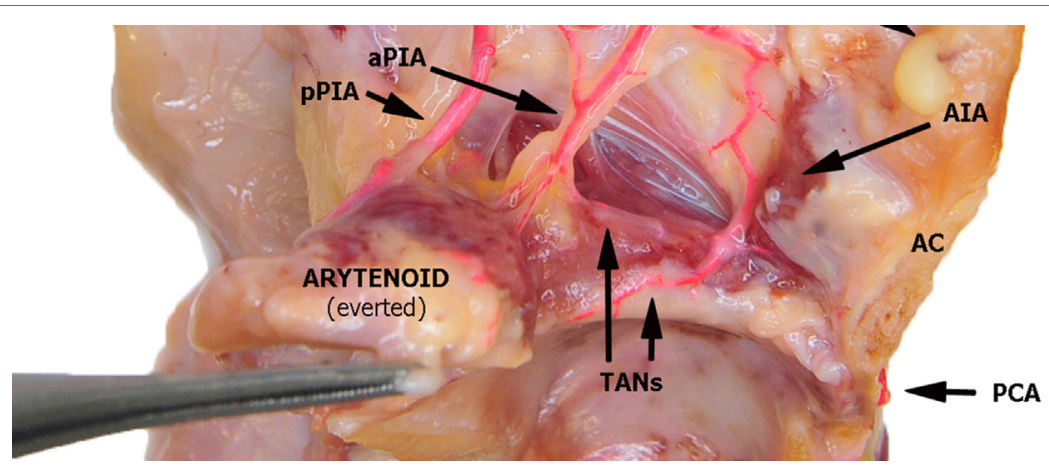

FIGURE 5 | Left fresh-frozen cadaver hemilarynx after midline section of the cricoid and thyroid cartilages, seen from above. The ipsilateral arytenoid had been everted while left aryepiglottic and false vocal folds had been removed. Paraglottic fat tissue and vocal muscle are still mostly in place. Bicomponent red silicone had been injected in the left common carotid and subclavian arteries. Abbreviations: AIA, antero-inferior artery; aPIA, anterior branch of the postero-inferior artery; pPIA, posterior branch of the postero-inferior artery; AC, anterior commissure; PCA, paracommissural branch of the crico-thyroid artery; TANs, lateral and medial transverse anastomotic networks. 
TABLE 1 | Summary of each vessel's detection rate, landmarks, and median diameter.

\begin{tabular}{lccc}
\hline Vessel & $\begin{array}{c}\text { Detection } \\
\text { rate (\%) }\end{array}$ & Landmarks & $\begin{array}{r}\text { Median } \\
\text { diameter (mm) }\end{array}$ \\
\hline Epiglottic artery & 100 & ea1 & 2.2 \\
& ea2 & 0.9 \\
\hline Antero-inferior artery & 95 & aia1 & 1.4 \\
& & aia2 & 0.7 \\
\hline Postero-inferior artery & 100 & - & 1.6 \\
PPIA & - & ppia & 1.4 \\
aPIA & - & apia & 0.9 \\
TAN1 & 100 & - & $>0.5$ \\
TAN2 & 91 & - & $>0.5$ \\
PCA & 100 & - & 1.6 \\
\hline
\end{tabular}

(11), and more rarely in three vessels (18\% of our series), but the areas of supply remain the same as previously described. During TLM, the most frequently encountered branch of the PIA is the aPIA, usually endangered at the level of the landmark apia during Type II or more advanced cordectomies (14), aimed at removing tissue in front of the vocal process and lateral to it in the vocal muscle. Its control is usually obtained by monopolar cauterization through insulated forceps. A more posterior resection encountering the PPIA is much rarer and mainly limited to arytenoidectomy and/or extensive aryepiglottic fold resection, where this branch may be transected at the level of our landmark ppia. Larger branches at the junction between ary- and pharyngoepiglottic folds usually require clipping, while more distal vessels are commonly coagulated by monopolar alone.

The AIA instead runs antero-inferiorly, with terminal vessels located in the AC area, receiving anastomotic branches from the PCA, as well as with TANs. The AIA subdivisions described by Imanishi and coworkers (and named A1, A2, and A3 in that paper) (11) were inconsistently represented and identified in our study, and probably correspond to branches with a diameter inferior to the critical size of $0.5 \mathrm{~mm}$. These vessels are usually encountered during Type Va and VI cordectomies at our landmark aia2 $(14,15)$, with the PCAs constantly found some millimeters apart from the midline, at the level of the AC. As noted, laser coagulation and/or monopolar cautery are usually sufficient to obtain bleeding control at this level. By contrast, moving downward to the anterior subcommissural level increases the risk of injuring larger branches of the crico-thyroid arteries and ILA at the level of the crico-thyroid membrane. These arteries may bleed profusely and sometimes require accurate clipping.

The TANs run parallel to the glottic plane on the two lateral (TAN1) and medial (TAN2) sides of the vocal muscle, connecting the PIA, AIA, and PCA. Even though they are usually larger than $0.5 \mathrm{~mm}$, monopolar cautery is usually sufficient to control bleeding (in particular at the level of the junction between the conus elasticus and vocal ligament).

The meandering course of laryngeal vessels in specific locations (EA, PIA, and TANs) observed in the present study is consistent with that already described by Iimura and coworkers on fixed specimens (10), and related to the peculiar movements of the underlying anatomical structures during swallowing and
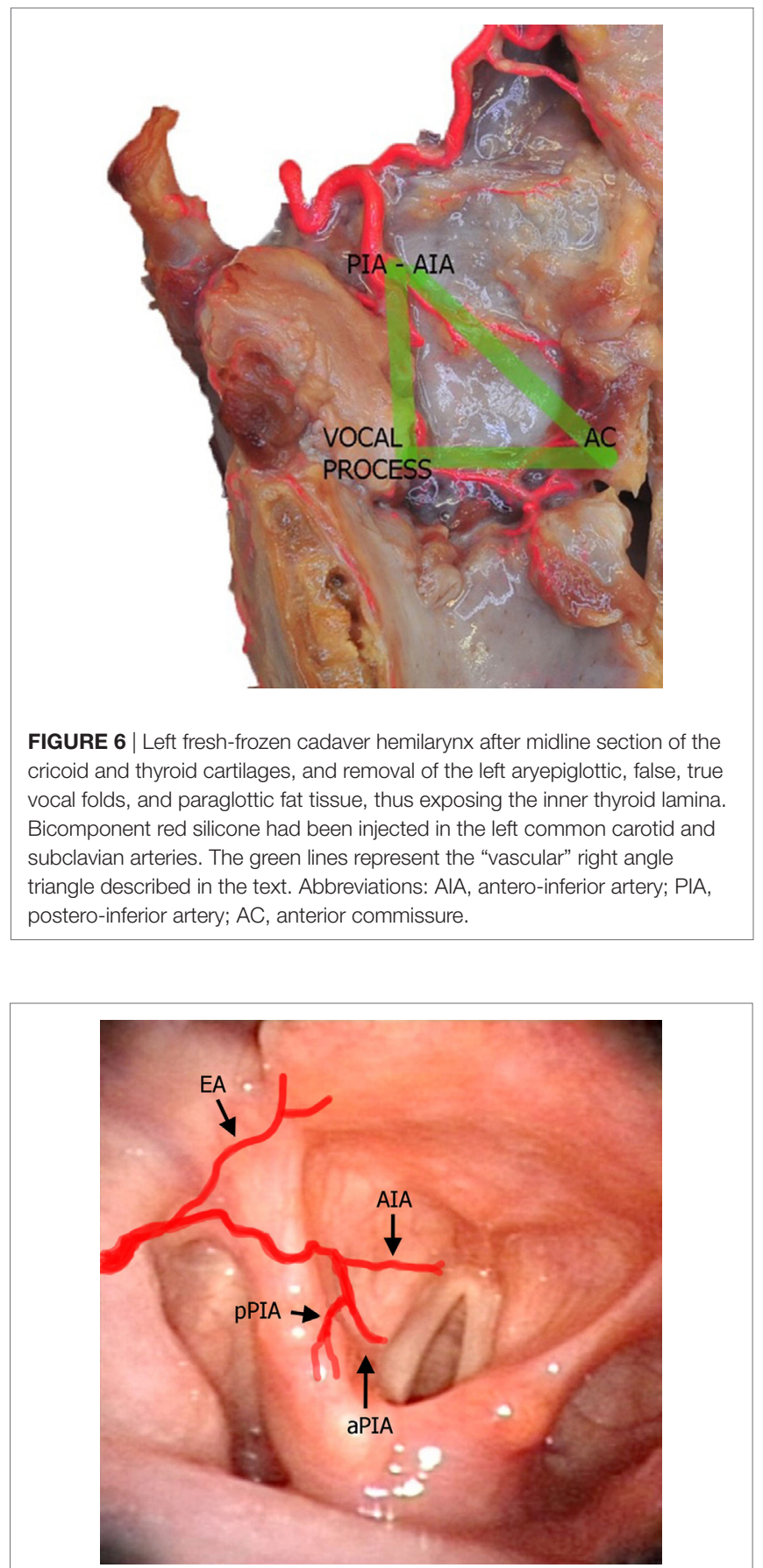

FIGURE 7 | Laryngeal videoendoscopy simulating the intraoperative transora surgical perspective, with superimposed red lines schematically representing the laryngeal arteries described in the text. Abbreviations: EA, epiglottic artery; AIA, antero-inferior artery; aPIA, anterior branch of the postero-inferior artery; pPIA, posterior branch of the postero-inferior artery.

phonation. However, we did not attempt any quantification of such a tortuous behavior of these vessels since without any practical consequence for the specific aim of our study.

In summary, two main arterial anastomotic high-flow areas can be, therefore, described in the endolarynx: (1) the AC, where 


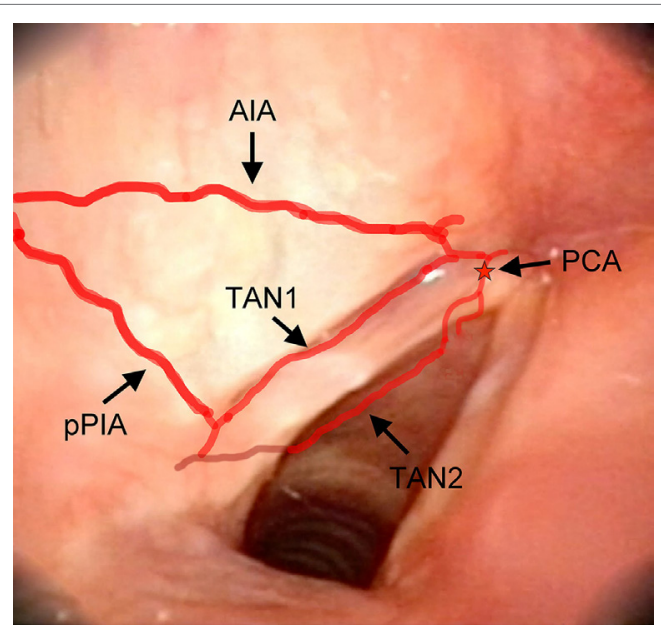

FIGURE 8 | Laryngeal videoendoscopy, more closely focusing on the glottic plane. Abbreviations: AIA, antero-inferior artery; pPIA, posterior branch of the postero-inferior artery; TAN1, lateral transverse anastomotic network; TAN2, medial transverse anastomotic network; red star, endolaryngeal emergence of the PCA, paracommissural branch of the crico-thyroid artery.

branches of the AIA, PCA, and TANs join together and (2) the vocal process, where the PPIA, aPIA, terminal branches arising from the ILA, and the TANs are intermingled. If, on the sagittal plane, we trace lines connecting each other these two areas and themselves with the entry point of the SLA, we obtain a right angle triangle: the vertical side corresponds to the PIA, the horizontal to the TANs, and the oblique to the AIA, while the vertices represent the most critical areas due to their higher hemorrhagic potential (Figure 6).

Some drawbacks must be acknowledged in the present study: first, even though fresh-frozen specimens are definitely closer to normal human anatomy than fixed ones, the use of a bicomponent red silicone filling the vessel lumens may partially change their size or course. Second, use of a micrometer caliper is less precise than making cross-sectional measurements under light microscopy within histologic sections. Third, the reference to specific landmarks chosen on the basis of macroscopic laryngeal anatomy

\section{REFERENCES}

1. Vilaseca-González I, Bernal-Sprekelsen M, Blanch-Alejandro JL, MoragasLluis M. Complications in transoral $\mathrm{CO}_{2}$ laser surgery for carcinoma of the larynx and hypopharynx. Head Neck (2003) 25:382-8. doi:10.1002/hed. 10207

2. Bernal-Sprekelsen M, Dazert S, Sudhoff H, Blanch J-L, Vilaseca I. Complications of transoral laser surgery for malignant tumors of the larynx and hypopharynx. Laryngorhinootologie (2009) 88:28-34. doi:10.1055/s-2008-1077786

3. Ellies M, Steiner W. Peri- and postoperative complications after laser surgery of tumors of the upper aerodigestive tract. Am J Otolaryngol (2007) 28:168-72. doi:10.1016/j.amjoto.2006.08.008

4. Chiesa Estomba CM, Reinoso FA, Velasquez AO, Fernandez JL, Conde JL, Hidalgo CS. Complications in $\mathrm{CO}_{2}$ laser transoral microsurgery for larynx carcinomas. Int Arch Otorhinolaryngol (2016) 20:151-5. doi:10.1055/s-0035-1569145

5. Hinni ML, Salassa JR, Grant DG, Pearson BW, Hayden RE, Steiner EW, et al. Transoral laser microsurgery for advanced laryngeal cancer. Arch Otolaryngol Head Neck Surg (2007) 133:1198-204. doi:10.1001/archotol.133.12.1198 may be only in part representative of the real surgical situation and are still prone to minimal shift due to specimen distortion (cadaver larynges were no more connected to their natural fixation points in the neck). However, we believe that this level of precision is sufficient for the specific and practical purposes of the present study (Figures 7 and $\mathbf{8}$ ). It is nonetheless possible that a larger study on more specimens could have been more sensitive in identifying potential vascular variants and abnormalities. However, the two more recent papers in the literature similar to the present were performed on comparable $(n=27)(10)$, or smaller $(n=6)$ (11) samples. Last but not least, the present study was carried on normal larynges, while surgery for laryngeal cancer must take into account the vascular alterations induced by the presence of the tumor itself. Larger, tortuous, more fragile, and aberrant vessels are frequently encountered when dealing with SCC, especially at the level of the supraglottis. Moreover, rarer histotypes (hemangiomas, paragangliomas, sarcomas, etc.) may present with atypical vascularization requiring special caution and adjunctive measures to prevent incontrollable bleeding.

\section{CONCLUSION}

The overall course, size, and distribution of the endolaryngeal vessels and their relationships with specific landmarks are described in the present anatomical cadaver study with the aim to provide surgeons with an instrument to guide them during the steep learning curve of transoral surgical approaches, in order to help in prevention and management of major intraoperative bleeding and troublesome postoperative hemorrhage.

\section{AUTHOR CONTRIBUTIONS}

PP, MF, NM, DL, AP, and FI: data collection and writing. BB, LR, and CP: data collection and revision.

\section{ACKNOWLEDGMENTS}

The authors acknowledge MedCure's for provision of anatomical specimens.

6. Oki T. The distribution of blood vessels in the larynx. Nichijibi (1958) 61:1827-41. doi:10.3950/jibiinkoka.61.1827

7. Pearson BW. Laryngeal microcirculation and pathways of cancer spread. Laryngoscope (1975) 85:700-13. doi:10.1288/00005537-197504000-00014

8. Trotoux J, Germain MA, Bruneau X. Vascularization of the larynx. Update of classical anatomic data from an anatomical study of 100 subjects. Ann Otolaryngol Chir Cervicofac (1986) 103:389-97.

9. Lang J, Nachbaur S, Fisher K, Vogel E. Uber den nervus larygeus superior und die arteria laryngea superior. Acta Anat (1987) 130:309-18. doi:10.1159/ 000146462

10. Iimura A, Itoh M, Terayama H, Nakamura T, He G, Kondo Y, et al. Anatomical study of meandering and functions of human intralaryngeal artery. Okajimas Folia Anat Jpn (2004) 81:85-92. doi:10.2535/ofaj.81.85

11. Imanishi N, Kondoh T, Kishi K, Aiso S. Angiographic study of the superior laryngeal artery. Okajimas Folia Anat Jpn (2009) 86:61-5. doi:10.2535/ofaj.86.61

12. Rusu MC, Nimigean V, Banu MA, Cergan R, Niculescu V. The morphology and topography of the superior laryngeal artery. Surg Radiol Anat (2007) 29:653-60. doi:10.1007/s00276-007-0267-4 
13. Goyal N, Yoo F, Setabutr D, Goldenberg D. Surgical anatomy of the supraglottic larynx using the da Vinci robot. Head Neck (2014) 36:1126-31. doi:10.1002/ hed. 23418

14. Remacle M, Eckel HE, Antonelli A, Brasnu D, Chevalier D, Friedrich G, et al. Endoscopic cordectomy. A proposal for a classification by the working committee, European Laryngological Society. Eur Arch Otorhinolaryngol (2000) 257:227-31. doi:10.1007/s004050050228

15. Remacle M, Van Haverbeke C, Eckel H, Bradley P, Chevalier D, Djukic V, et al. Proposal for revision of the European Laryngological Society classification of endoscopic cordectomies. Eur Arch Otorhinolaryngol (2007) 264:499-509. doi:10.1007/s00405-007-0304-2
Conflict of Interest Statement: The authors declare that the research was conducted in the absence of any commercial or financial relationships that could be construed as a potential conflict of interest.

Copyright (c) 2018 Perotti, Ferrari, Montalto, Lancini, Paderno, Incandela, Buffoli, Rodella and Piazza. This is an open-access article distributed under the terms of the Creative Commons Attribution License (CC BY). The use, distribution or reproduction in other forums is permitted, provided the original author(s) and the copyright owner are credited and that the original publication in this journal is cited, in accordance with accepted academic practice. No use, distribution or reproduction is permitted which does not comply with these terms. 


\title{
A Contemporary Review of Evidence for Transoral Robotic Surgery in Laryngeal Cancer
}

\author{
Philippe Gorphe* \\ Department of Head and Neck Oncology, Institute Gustave Roussy, Université Paris-Saclay, Villejuif, France
}

Numerous studies have shown that transoral robotic surgery (TORS) for oropharyngeal cancers is safe and that it yields satisfactory functional and oncological outcomes. For many teams worldwide, it is therefore a standard surgical approach with eligible patients. In the same time, TORS is increasingly being used and described in the context of laryngeal cancer surgery. It is proposed as an alternative to open approaches, which may yield inconsistent functional results and significant rates of postoperative complications. It may also be an alternative to definitive radiotherapy, which entails significant early and late toxicities. Moreover, it has been explored as an alternative to endoscopic laser surgery in patients with difficult exposure, even though there is still a lack of evidence about which procedure provides better vizualization of the vocal cords. This article provides

OPEN ACCESS

Edited by:

Giorgio Peretti,

Università di Genova, Italy

Reviewed by:

Mohssen Ansarin,

Istituto Europeo di Oncologia

s.r.l., Italy

Marc Joseph Remacle,

Centre Hospitalier de Luxembourg,

Luxembourg

*Correspondence:

Philippe Gorphe

philippe.gorphe@gustaveroussy.fr

Specialty section:

This article was submitted

to Head and Neck Cancer,

a section of the journal

Frontiers in Oncology

Received: 06 February 2018

Accepted: 04 April 2018

Published: 18 April 2018

Citation:

Gorphe P (2018) A Contemporary

Review of Evidence for Transoral

Robotic Surgery in Laryngeal Cancer.

Front. Oncol. 8:121.

doi: 10.3389/fonc.2018.00121 a review of the indications for TORS in laryngeal cancer, the peri-operative morbidity, functional outcomes, and oncological results.

Keywords: transoral robotic surgery, laryngeal neoplasms, TORS, supraglottic laryngectomy, total laryngectomy, glottic cordectomy

\section{INTRODUCTION}

The underlying principle of transoral robotic surgery (TORS) is to be able to reliably perform state-of-the-art oncological resection of the primary tumor through a minimally invasive transoral approach (1). Surgery is assisted by remote-controlled miniaturized surgical instruments and magnified visualization with a high-definition three-dimensional camera. It has proven to be an effective alternative to open surgery, with or without a mandibulotomy approach for oropharyngeal cancer in a large number of studies since its approval by the FDA in 2009 for T1 and T2 lesions. Series showed decreased rates of postoperative complications, improved functional outcomes, and favorable oncological results (2). For a large number of authors, it has become a standard-of-care among other treatment modalities in oropharyngeal squamous cell carcinoma staged T1-T2 (1, $3-5)$. With laryngeal cancers, TORS has been explored as minimally invasive surgery for supraglottic and glottic lesions, as well as for total laryngectomy. This article provides a review of the evidence available to date in regard to indications for TORS in laryngeal cancer, peri-operative morbidity, functional outcomes, and oncological results.

\section{TRANSORAL ROBOTIC SUPRAGLOTTIC LARYNGECTOMY}

\section{Indications}

Supraglottic laryngectomy accounts for the vast majority of transoral robotic surgical procedures published in regard to laryngeal cancer. Transoral robotic supraglottic laryngectomy (TORS-SGL) must allow for complete oncological resection of the supraglottic tumor, while it must also preserve 
anatomical and neurophysiological functions of the glottic larynx (i.e., protective, respiratory, and phonatory functions) and of the base of the tongue. For these reasons, the preferential indications are selected T-stage T1 and T2, and a few T3 cancers (6). The three following groups of tumors have been reported to be potentially suitable for open supraglottic laryngectomy; TORS-SGL must be assessed for each group according to their specific local extensions (7).

The first group encompasses the early T-stage tumors of the anterior epilarynx (suprahyoid epiglottis) and lateral epilarynx (laryngeal aspects of the aryepiglottic folds). The extent of resection in transoral procedures depends mainly on local invasion of the vallecula and of the lateral pharynx through the threefold region. Small tumors originating from the antero-lateral epilarynx are amenable to partial surgery with good functional outcomes and local control. When the hyoid bone is not directly involved with tumor and the vallecula mucosa and tongue base are uninvolved, the feasibility of hyoid bone preservation has been demonstrated in literature from open supraglottic laryngectomy, from transoral laser microsurgery (TLM), and from TORS-SGL $(6,8-10)$. Tumors of the posterior epilarynx (arytenoids) carry an increased risk of postoperative aspiration and swallowing difficulties. They are challenging cases and they should therefore only be performed by the most experienced surgeons. The second group includes tumors of the anterior laryngeal vestibule (infrahyoid epiglottis). In these tumors, the risk of local extension into the pre-epiglottic space (PES) argues for complete resection of this space (11). Moreover, tumors of the infrahyoid epiglottis may extend superficially to ventricular bands and they may require their resection along with resection of the paraglottic space above the plane of the ventricle. In the third group that comprises the early T-stage tumors of the vallecula (12), surgeons need be careful when considering TORS-SGL. Potential local extensions that are challenging for a transoral robotic approach are deep involvement of the muscles of the base of tongue, and lateral submucosal extensions to the pharyngoepiglottic and aryepiglottic folds. Frozen sections should be routinely performed to ensure complete resection.

In the literature to date, $35 \%$ of the published patients operated on by TORS-SGL with available clinical staging were reported to have a cT1 disease, $53.5 \%$ had a cT2 disease, and $11 \%$ had a cT3 cancer (13-25). Twenty-three patients were reported to have been operated on for salvage surgery after radiotherapy $(22,25$, 26). Few details were provided in series regarding the precise disease location and the extensions for each patient, although the epiglottis was involved in the vast majority of the patients with an available clinical description. Unfortunately, for most of the patients it was not possible to distinguish between suprahyoid tumors, infrahyoid tumors, and tumors that involved both sites of the epiglottis.

\section{Contraindications}

Absolute contraindications to TORS-SGL comprise: insufficient transoral exposure (e.g., interincisor distance $<3 \mathrm{~cm}$, trismus, and difficult exposure); invasion of the thyroid and/or cricoid cartilage; impaired vocal cord mobility or arytenoid mobility; invasion of the paraglottic space; posterior commissure invasion; extension to the glottic larynx; involvement of more than $2 \mathrm{~cm}$ of the base of the tongue mucosa or invasion of the tongue base muscles. Relative contraindications to TORS-GL include pulmonary disease and a respiratory insufficiency given the risk of postoperative aspirations. Moreover, elderly patients should be carefully evaluated for their performance status.

\section{Surgical Procedure}

The following three steps are the main components of the standard complete TORS-SGL for a supraglottic laryngeal cancer (13, 27). They can be performed in any order, provided that exposure remains adequate and allows for a complete visualization of the tumor for oncological monobloc resection (25). For tumors of the vallecula, a transvallecular approach is to be replaced by incision of the tongue base. Weinstein et al. recommend beginning with splitting of the suprahyoid epiglottis, sectioning the epiglottis vertically up to the petiole, then performing each side of the supraglottic laryngectomy one by one (13).

A transvallecular approach to the PES is initiated by transection of the vallecular mucosa, identification of the hyoid bone, dissection of the thyro-hyoid membrane, and identification of the thyroid cartilage. Laterally, superior laryngeal vascular bundles are encountered in the dissection of the lateral pharyngoepiglottic folds, and they are clipped. Dissection of the PES is then performed.

Aryepiglottic folds are transected superiorly and laterally to the arytenoid. The dissection is pursued between the arytenoid cartilages and ventricular bands to the level of the ventricles, allowing resection of the false vocal cords and the upper paraglottic spaces above the ventricles within the specimen.

Horizontal sectioning goes through the ventricles, exposing the ventricular floors, and the vocal cords. Anteriorly, the petiole is transected just above the anterior commissure, and the transection is connected to the dissection of the lower PES.

After completion of these three steps, the specimen can readily be removed and the margins are determined from frozen sections if necessary (Figure 1). In almost all of the cases reported in literature, the patient selection and the exposure were sufficient to ensure complete oncological resection via the transoral robotic approach. Only two patients were reported to have been converted to an open approach due to limited exposure and dissection $(19,25)$. Neck dissections can be performed during the same procedure. For some authors, neck dissections

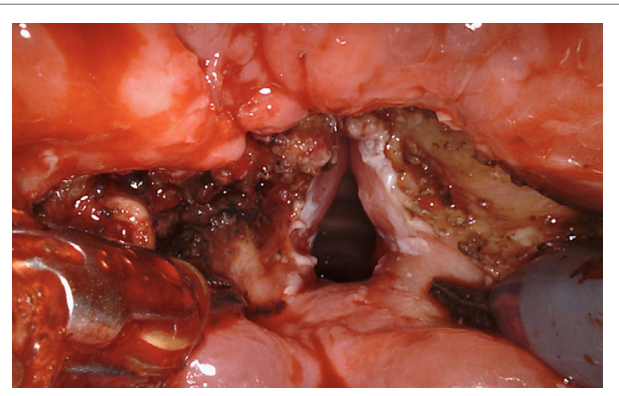

FIGURE 1 | Transoral robotic supraglottic partial laryngectomy in a patient with epiglottic cancer. 
are performed secondary within the first 3 weeks following the supraglottic laryngectomy, to limit the edema and the need for a tracheotomy $(13,18,21,28-30)$.

\section{Peri-Operative Outcomes}

The reported rates of postoperative complications are low. Mendelsohn et al. reported 5 instances of temporary vocal cord hypomobility out of 18 patients (17); Lallemant et al. reported bleeding at day 2 in 1 patient out of 23 patients (16); Alon et al. reported a skin thermal injury with late laryngeal stenosis (14); while Kayhan et al. reported two postoperative pulmonary infections and one late laryngeal stenosis (21).

In several series, extubation of patients occurred at least $25 \mathrm{~h}$ after the surgery $(18,21,22)$. TORS supraglottic laryngectomy could be performed without a tracheotomy in most of the patients. In the largest series published to date, only 12 patients out of 84 (14\%) required a per-operative tracheotomy (25). However, eight patients required a secondary tracheotomy for postoperative edema. Only 1 tracheotomy out of 84 patients was definitive. The management of postoperative nutrition varies between authors; some authors have published oral feeding as soon as day 1 (19); however, the postoperative period was generally assisted with nasogastric feeding, and for most of authors oral intake was resumed between 7 and 10 days on average $(13,16,17,25,31)$.

\section{Oncological Outcomes}

When reported, the rate of complete resection with free margins was between 60 and $100 \%$ in series comprising at least 10 patients $(16,17,20,25,31,32)$. The rates of postoperative radiotherapy ranged from 40 to $70 \%(16,17,20,25,31,33)$. The rates of local recurrence when reported ranged from 0 to $11 \%$ (34). The 2-year overall survival were reported in two series and ranged from 66.7 to $88.9 \%(15,17)$.

\section{TRANSORAL ROBOTIC TOTAL LARYNGECTOMY}

\section{Indications}

Transoral robotic total laryngectomy (TORS-TL) has been shown to be feasible, although only a very small number of patients have been operated on by this method to date (35-38). While yet to be proven, the reported objective is to decrease postoperative morbidity; first, by decreasing the risk of postoperative fistula as a result of better preservation of the pharyngeal mucosa; secondly, by limiting the lateral dissection between the pharyngeal and vascular spaces, thus decreasing the risk of a carotid blow-out. However, the vast majority of patients who require a primary total laryngectomy present with a locally advanced squamous cell carcinoma of the larynx, and they therefore require a bilateral neck dissection concurrent with the laryngectomy. The benefits of a minimally invasive approach to the laryngeal site in these patients are therefore somewhat questionable, and precise indications have yet to be well-defined.

The small number of surgeons experienced with this procedure recommends that TORS-TL be evaluated in the following three indications where neck dissections can be avoided (35-38).
The first indication is salvage surgery for locally limited failure of the primary lesion after radiotherapy or chemoradiotherapy that is not amenable to a salvage partial laryngectomy due to local or general contraindications. Naturally, the primary pharyngeal closure must be readily achievable and there should not be a need for a flap closure. Preservation of the infrahyoid muscles must be oncologically possible without incurring risk, meaning that there must not be any doubt about integrity of the thyroid cartilage or the cricothyroid ligament. In practice, this situation is rare in a salvage context. The second and even rarer indication is for benign or malignant laryngeal tumors with limited local extensions requiring a primary total laryngectomy for oncological or functional reasons, while not requiring extensive perilaryngeal dissection. Potential rare and various histologies encompass adenoid cystic carcinoma, low-grade chondrosarcoma, and chondroma, among others. The third indication is refractory laryngeal dysfunction with long-term tracheotomy and enteral feeding. Such indications are encountered in patients with a neurodegenerative disease, in patients with definitive highgrade sequelae from a previous laryngeal trauma who generally have undergone multiple operations prior to the laryngectomy decision, and in patients with severe chronic post-radiotherapy toxicity, with or without chondronecrosis. However, careful patient selection is required in light of the potential for associated pharyngeal dysfunction, as a pharyngeal stenosis would not be improved without mucosa repair with a flap.

Transoral robotic total laryngectomy has been reported in the literature for series that comprised multiple cancer localizations and various robotic surgical procedures (39). However, only three publications to date have comprised series of patients operated on with TORS-TL, with the total number of patients limited to 15 (36-38). Notably, two patients eventually had unsuccessful exposure for complete robotic resection. Six patients (40\%) had a local squamous cell carcinoma recurrence after radiotherapy; three patients (20\%) had a primary surgery for a rare endolaryngeal tumor, comprising two low-grade chondrosarcoma and one cystic adenoid carcinoma; and six patients (40\%) had a functional total laryngectomy for a chronic laryngeal dysfunction, due to sequelae from radiotherapy in three patients, a neurodegenerative disease in one patient, an idiopathic bilateral vocal cord palsy in one patient, and due a multioperated laryngeal stenosis in another patient.

\section{Procedure}

The first part of the procedure is performed via a minimal open approach of the neck. A skin excision is made in front of the future stoma, and the thyroid isthmus is divided. The trachea is sectioned, the tracheal part of the specimen is dissected from the esophagus to facilitate its superior mobilization during the transoral dissection, and the inferior side of the stoma is sutured.

The second part of the procedure begins as a transoral robotic supraglottic laryngectomy. The pharyngoepiglottic folds are divided, superior laryngeal vascular bundles are clipped, and the mucosa of the vallecula is incised. This allows for exposure of the superior side of the hyoid bone, which is resected within the specimen in TORS-TL. The anterior side of the hyoid bone is then followed, and dissection of the pre-epiglottic fat may or 
may not be carried out according to the surgical indications. The thyro-hyoid membrane is dissected, until the upper side of the thyroid cartilage can be identified. The thyroid alas are freed by dissection and moved posteriorly, while preserving the outer perichondrium along with the infrahyoid muscles. On each side the anterior pyriform sinus mucosa is incised as close to the larynx as possible and the lateral pyriform sinus is mobilized, allowing dissection of the constrictors after transection of the lateral thyro-hyoid ligaments (laryngeal suspensory ligaments). Finally, the postcricoid mucosa is sectioned under direct transoral visualization, and the specimen is freed via the oral cavity (Figure 2). The pharynx is closed with a single horizontal suture line if possible, although it can also be sutured to the preserved strap muscles in a U-shaped configuration if necessary. Of note, Krishnan et al. have reported that they prefer removing the larynx through the cervical opening rather than through the oral cavity (38). In their opinion, transoral delivery of the larynx was associated with dilatation of the pharyngeal defect and it resulted in a larger neopharynx to be closed.

A technical limitation of TORS-TL that has been reported by several authors is exposure of the larynx during the transoral procedure. Exposure has been described in some cases to be insufficient. In the series of Dowthwaith et al., one patient out of three had an inadequate transoral exposure (36); in the series of Smith et al., two patients out of seven had an inadequate transoral exposure (37); while Krishnan et al. did not report any difficulty among five patients (38).

\section{Peri-Operative Outcomes}

Postoperative pharyngocutaneous fistulas were reported in three patients: two patients underwent salvage TORS-TL after failure of radiotherapy, and one patient underwent a functional TORS-TL for a multioperated laryngeal stenosis $(37,38)$. The rate of postoperative pharyngocutaneous fistulas was 0-28.6\% (36-38). One patient in the series of Dowthwaith et al. had bleeding of the pharyngeal suture on day 9 after resumption of oral intake, and he required transoral cauterization (36). Other patients in their series resumed oral intake on day 7 and day 8 . In the series of Krishnan et al., oral intake was resumed on day 10 and day 12 for the two patients who were not percutaneous enteric gastrostomy dependent for nutrition prior to the TORS-TL (38).

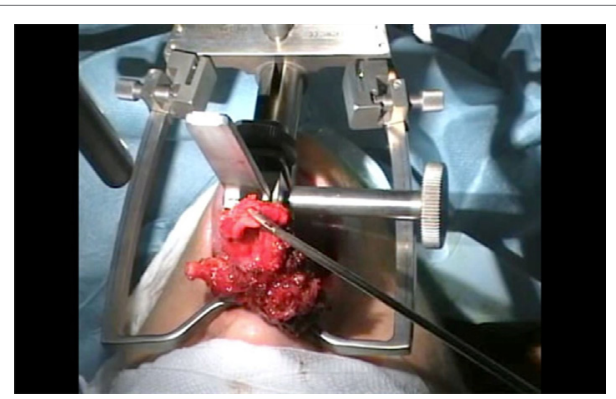

FIGURE 2 | Transoral removal of the larynx with the robot moved out of position and the Faye-Kastenbauer mouth retractor in place.

\section{Oncologic Outcomes}

Krishnan et al. reported free margins for the three patients operated on due to a cancer in their series. The first patient had an rT2N0M0 squamous cell carcinoma, the second patient had an adenoid cystic carcinoma, and the third patient had a low-grade chondrosarcoma. They were still alive and disease-free 54, 54, and 18 months, respectively, after the surgery.

\section{TRANSORAL ROBOTIC GLOTTIC CORDECTOMY}

\section{Indications}

Transoral laser microsurgery is established as a standard-ofcare for minimal-invasive surgery in early glottic cancer. In supraglottic cancer, it is an alternative that yields similar oncological outcomes to open partial laryngectomy and definitive radiotherapy in selected indications $(40,41)$. However, adequate exposure of the glottic larynx is sometimes a serious limitation in TLM in a small number of patients with early-stage neoplasms, despite numerous refinements to the procedure by experienced teams (42). The feasibility of TORS in glottic surgery was first demonstrated by O'Malley et al. in 2006 in a canine model (43). In 2009, Park et al. further published the feasibility results for three patients operated on with TORS for a glottic lesion (44); one had a stage rT2N2b glottic carcinoma involving the anterior commissure, one had a stage $\mathrm{rT} 1 \mathrm{bN} 0$ recurrent glottic carcinoma after radiotherapy involving the anterior commissure, and one had a glottic melanoma. An improvement of the surgical procedure using $\mathrm{CO}_{2}$ fiber was subsequently described by Blanco et al. (45), allowing adequate oncological resection of a Tla glottic carcinoma with good functional outcomes. In 2012, Vural et al. reported a successful TORS glottic resection that removed the complete paraglottic spaces up to the upper border of cricoid cartilage (46). The patient had a recurrent carcinoma of the glottis involving the anterior commissure with impaired vocal cord mobility and a small subglottic anterior extension, 5 years after TLM and adjuvant radiotherapy. The authors referred to the procedure as a supracricoid laryngectomy with preservation of the cartilage framework, because of resection of the paraglottic spaces. However, the epiglottis above the anterior commissure resection was preserved, as was hence the PES. This was the largest glottic procedure case published to date. Since then, four series of patients with early-stage glottic cancer operated on with TORS have been published $(16,32,47,48)$.

In 2012, Kayhan et al. published a series of 10 patients operated on with TORS for T1 glottic cancer (47). The lesions were not described, although they thought to have been stage T1a, as the reported procedure was robotic cordectomy, in patients that were provided therapeutic alternatives including radiotherapy and TLM. Lallemant et al. further described a series of 13 patients (16) operated on with TORS for glottic carcinoma, stage T1a in 6 patients, T1b in 6 patients, and a T2 glotto-supraglottic lesion due to extension to the petiole in 1 patient. The same year, De Virgilio et al. published a review of strategies used at their center to improve TORS exposure (32), including 18 patients operated for glottic carcinoma. Ten patients were stage T1, of 
whom one had a recurrent disease, and eight patients were stage T2, without providing more details. Recently, Wang et al. described a series of eight patients operated on with TORS for glottic carcinoma with anterior commissure involvement (48), that was either stage T1 $(n=3)$ or stage T2 $(n=5$, of whom two had a recurrent disease after previous TLM). Therefore, an extension to the anterior commissure appears to be surgically resectable via a TORS approach, although the management of thyroid cartilage remains an issue in these patients irrespective of the procedure.

\section{Peri-Operative Outcomes}

All of the patients in the published series had a successful procedure, including adequate exposure and satisfactory resection $(16,32,47,48)$. The frozen sections were free of cancer $(47,48)$. Kayhan et al. reported that 1 patient out of 10 was tracheotomized for 3 days becdue to chronic obstructive pulmonary disease (47). Lallemant et al. reported one secondary tracheotomy until day 15 after the surgery (16), due to a cervical emphysema and a pneumothorax, along with limited laryngeal bleeding due to a small unnoticed breach of the cricothyroid ligament during anterior commissure resection.

Except for the tracheotomized patient who required a nasogastric tube for 5 days, oral intake was successfully resumed 6-24 $\mathrm{h}$ following the surgery in all of the patients for Kayhan et al. (47). In the series of Lallemant et al. (16), aside from the tracheotomized patient who required a nasogastric tube for 18 days, two patients required a nasogastric tube for either 3 or 10 days, and 10 patients resumed oral intake on the first day following the surgery. In the series of Wang et al. (48), including patients with a larger resection that encompassed the anterior commissure, one patient resumed oral intake on the first day following the surgery, while all of the other patients required a nasogastric tube, for a mean duration of 14 days.

\section{Oncologic Outcomes}

Kayhan et al. reported neither the definitive status of the margins nor the postoperative adjuvant treatment; no recurrences occurred during follow-up with a mean duration of 9 months (47). In the series of Lallemant et al., 6 patients out of 13 had a sufficient resection (16), 3 patients had a microscopically positive margin or a close margin of less than $1 \mathrm{~mm}$, and 4 patients had unclassifiable margins due to thermal injury. None of the patients received adjuvant treatment. Two patients out of the $13(15.4 \%)$ had a local recurrence. One local recurrence occurred 10 months after the TORS for a T1b lesion with microscopic margins of less than $1 \mathrm{~mm}$. It was staged $\mathrm{rT} 1$ and it was treated with radiotherapy. The other local recurrence occurred 12 months after the TORS for a T1a lesion with unclassifiable margins. It was staged rT4a, and it was treated by a salvage total laryngectomy. De Virgilio et al. reported that all of the margins were free of disease (32). Wang et al. (48) reported that three patients out of eight had microscopically positive margins on the specimen. However, all of these patients were spared postoperative radiotherapy as the definitive pathological examinations of all of the frozen specimens were negative and follow-up examinations did not reveal any suspicious lesions. The mean follow-up period was 40 months, without local recurrence. The 3-year local control, laryngeal preservation, and overall survival were $100 \%$.

\section{LARYNGEAL EXPOSURE WITH THE FLEX ROBOTIC SYSTEM}

All series reported in the previous sections have been performed using the da Vinci Surgical System (Intuitive Surgical, Sunnyvale, CA, USA). The endowrist instruments and the $30^{\circ}$ endoscopic three-dimensional camera are technological improvements that demonstrated very useful for most of transoral procedures. However, the da Vinci System was designed for work in a large cavity as in thoracosopic or laparoscopic surgery, and limitations have been reported when transposed in transoral laryngeal surgery. For Remacle et al., the main difficulty is getting a good vizualization and exposure of the larynx due to the bend around the tongue base, because of rigid robotic arms and endoscopes (49). Furthermore, the limited number of cutting devices is an issue for glottic surgery, notably the lack of available $\mathrm{CO}_{2}$ laser fiber $(16,47,50)$. The Flex Robotic System (Medrobotics, Raynham, MA, USA) is a new flexible robotic scope that achieved CE mark in 2014 and FDA clearance in 2015. The system consists of a Flex Scope, with flexible instruments inserted through lateral guide tubes along the flexible endoscope and handed directly by the surgeon, and a Flex Console, which controls the position and mobility of the flexible endoscope HD camera and holds the console display (51). The first patients were treated in Europe in June 2014 and the Flex System has demonstrated its feasibility for transoral surgery (51-53). More recently, an European prospective non-randomized multicentric study has established the safety and efficacy of the Flex System for transoral robotic head and neck surgery in 79 patients with a combination of various procedures and lesions $(54,55)$. Of these patients, 21 underwent TORS with the Flex System for a laryngeal lesion: 11 lesions of the epiglottis, 2 lesions of the ventricular folds, 3 lesions of the arytenoid cartilages, and 5 lesions of the vocal cords. No detail was given regarding the benign or malignant nature of the lesions, nor regarding the procedures performed. Lang et al. reported that the endolarynx exposure was feasible, but that some limitations remained with respect to adequate triangulation for precise resection of smaller lesions inside the larynx (55). Matheis et al. published a focused description of their single-center experience of 40 patients who underwent TORS with the Flex System (54). Sixteen patients had a laryngeal supraglottic lesion, 12 benign and 4 malignant. An oncologic resection was planned and successfully performed in three patients with an epiglottic carcinoma staged T1 $(n=1)$ or T2 $(n=2)$. The fourth malignant lesion was reported to be a T2 laryngeal carcinoma, and the patient underwent TORS with the Flex System for endoscopy and biopsy. The authors reported that lesions of the ventricular fold could not be visualized properly in two patients because of unfavorable narrow anatomy, while the transoral microscopic approach in these two patients was successful. Thus, the Flex System has demonstrated to be a safe and effective device in TORS, but experience in oncologic resection of laryngeal neoplasms is still in the early stages. Furthermore, some technical limitations remain as to adequate endolaryngeal 
exposure for oncologic surgery, which warrants further technological developments.

\section{CONCLUSION}

Transoral robotic surgery for laryngeal cancer has been shown to be feasible for minimally invasive partial laryngectomy for either supraglottic or glottic cancer, as well as for total laryngectomy, in selected patients. However, the level of evidence for oncological safety as compared with other conventional treatment modalities remains low due to the small number of published series to date and the lack of randomized trials. Furthermore, it is still to be

\section{REFERENCES}

1. Holsinger FC, Ferris RL. Transoral endoscopic head and neck surgery and its role within the multidisciplinary treatment paradigm of oropharynx cancer: robotics, lasers, and clinical trials. J Clin Oncol (2015) 33(29):3285-92. doi:10.1200/JCO.2015.62.3157

2. Weinstein GS, O’Malley BW Jr, Magnuson JS, Carroll WR, Olsen KD, Daio L, et al. Transoral robotic surgery: a multicenter study to assess feasibility, safety, and surgical margins. Laryngoscope (2012) 122(8):1701-7. doi:10.1002/ lary. 23294

3. Sload R, Silver N, Jawad BA, Gross ND. The role of transoral robotic surgery in the management of HPV negative oropharyngeal squamous cell carcinoma. Curr Oncol Rep (2016) 18(9):53. doi:10.1007/s11912-016-0541-x

4. Weinstein GS. Transoral robotic surgery and the standard of care. Int J Radiat Oncol Biol Phys (2017) 97(1):4. doi:10.1016/j.ijrobp.2016.09.028

5. Genden EM, O’Malley BW Jr, Weinstein GS, Stucken CL, Selber JC, Rinaldo A, et al. Transoral robotic surgery: role in the management of upper aerodigestive tract tumors. Head Neck (2012) 34(6):886-93. doi:10.1002/hed.21752

6. Smith RV. Transoral robotic surgery for larynx cancer. Otolaryngol Clin North Am (2014) 47(3):379-95. doi:10.1016/j.otc.2014.03.003

7. Succo G, Peretti G, Piazza C, Remacle M, Eckel HE, Chevalier D, et al. Open partial horizontal laryngectomies: a proposal for classification by the working committee on nomenclature of the European Laryngological Society. Eur Arch Otorhinolaryngol (2014) 271(9):2489-96. doi:10.1007/s00405-014-3024-4

8. Rodrigo JP, Suarez C, Silver CE, Rinaldo A, Ambrosch P, Fagan JJ, et al. Transoral laser surgery for supraglottic cancer. Head Neck (2008) 30(5):658-66. doi:10.1002/hed.20811

9. Silver CE, Beitler JJ, Shaha AR, Rinaldo A, Ferlito A. Current trends in initial management of laryngeal cancer: the declining use of open surgery. Eur Arch Otorhinolaryngol (2009) 266(9):1333-52. doi:10.1007/s00405-009-1028-2

10. Timon CI, Gullane PJ, Brown D, Van Nostrand AW. Hyoid bone involvement by squamous cell carcinoma: clinical and pathological features. Laryngoscope (1992) 102(5):515-20. doi:10.1288/00005537-199205000-00008

11. Lee NK, Goepfert H, Wendt CD. Supraglottic laryngectomy for intermediate-stage cancer: U.T. M.D. Anderson Cancer Center experience with combined therapy. Laryngoscope (1990) 100(8):831-6. doi:10.1288/00005537199008000-00007

12. Laccourreye L, Garcia D, Menard M, Brasnu D, Laccourreye O, Holsinger FC. Horizontal supraglottic partial laryngectomy for selected squamous carcinoma of the vallecula. Head Neck (2008) 30(6):756-64. doi:10.1002/ hed. 20780

13. Weinstein GS, O’Malley BW Jr, Snyder W, Hockstein NG. Transoral robotic surgery: supraglottic partial laryngectomy. Ann Otol Rhinol Laryngol (2007) 116(1):19-23. doi:10.1177/000348940711600104

14. Alon EE, Kasperbauer JL, Olsen KD, Moore EJ. Feasibility of transoral robotic-assisted supraglottic laryngectomy. Head Neck (2012) 34(2):225-9. doi:10.1002/hed.21719

15. Olsen SM, Moore EJ, Koch CA, Price DL, Kasperbauer JL, Olsen KD. Transoral robotic surgery for supraglottic squamous cell carcinoma. Am J Otolaryngol (2012) 33(4):379-84. doi:10.1016/j.amjoto.2011.10.007

16. Lallemant B, Chambon G, Garrel R, Kacha S, Rupp D, Galy-Bernadoy C, et al. Transoral robotic surgery for the treatment of T1-T2 carcinoma of the proved that TORS provide oncological and functional outcomes comparable with TLM. Another major issue with TORS in laryngeal cancer is the underlying necessity for access and adequate exposure, as instrument size and individual patient anatomy can present serious limitations. Future technological developments and miniaturization should improve the feasibility in a larger number of patients.

\section{AUTHOR CONTRIBUTIONS}

The author confirms being the sole contributor of this work and approved it for publication.

larynx: preliminary study. Laryngoscope (2013) 123(10):2485-90. doi:10.1002/ lary. 23994

17. Mendelsohn AH, Remacle M, Van Der Vorst S, Bachy V, Lawson G. Outcomes following transoral robotic surgery: supraglottic laryngectomy. Laryngoscope (2013) 123(1):208-14. doi:10.1002/lary.23621

18. Oysu C, Sahin-Yilmaz A. En bloc resection of epiglottic tumors with transoral robotic approach-preliminary results. Int J Med Robot (2013) 9(4):477-9. doi:10.1002/rcs.1516

19. Ozer E, Alvarez B, Kakarala K, Durmus K, Teknos TN, Carrau RL. Clinical outcomes of transoral robotic supraglottic laryngectomy. Head Neck (2013) 35(8):1158-61. doi:10.1002/hed.23101

20. Park YM, Kim WS, Byeon HK, Lee SY, Kim SH. Surgical techniques and treatment outcomes of transoral robotic supraglottic partial laryngectomy. Laryngoscope (2013) 123(3):670-7. doi:10.1002/lary.23767

21. Kayhan FT, Kaya KH, Altintas A, Sayin I. Transoral robotic supraglottic partial laryngectomy. J Craniofac Surg (2014) 25(4):1422-6. doi:10.1097/ SCS.0000000000000572

22. Perez-Mitchell C, Acosta JA, Ferrer-Torres LE. Robotic-assisted salvage supraglottic laryngectomy. P R Health Sci J (2014) 33(2):88-90.

23. Vicini C, Leone CA, Montevecchi F, Dinelli E, Seccia V, Dallan I. Successful application of transoral robotic surgery in failures of traditional transoral laser microsurgery: critical considerations. ORL J Otorhinolaryngol Relat Spec (2014) 76(2):98-104. doi:10.1159/000359953

24. Mendelsohn AH, Remacle M. Transoral robotic surgery for laryngeal cancer. Curr Opin Otolaryngol Head Neck Surg (2015) 23(2):148-52. doi:10.1097/ MOO.0000000000000144

25. Razafindranaly V, Lallemant B, Aubry K, Moriniere S, Vergez S, Mones ED, et al. Clinical outcomes with transoral robotic surgery for supraglottic squamous cell carcinoma: experience of a French evaluation cooperative subgroup of GETTEC. Head Neck (2016) 38(Suppl 1):E1097-101. doi:10.1002/ hed. 24163

26. Meulemans J, Vanclooster C, Vauterin T, D’Heygere E, Nuyts S, Clement PM, et al. Up-front and salvage transoral robotic surgery for head and neck cancer: a Belgian multicenter retrospective case series. Front Oncol (2017) 7:15. doi:10.3389/fonc.2017.00015

27. Durmus K, Gokozan HN, Ozer E. Transoral robotic supraglottic laryngectomy: surgical considerations. Head Neck (2015) 37(1):125-6. doi:10.1002/ hed. 23645

28. Muderris T, Bercin S, Sevil E, Acar B, Kiris M. Transoral robotic surgery for atypical carcinoid tumor of the larynx. J Craniofac Surg (2013) 24(6):1996-9. doi:10.1097/SCS.0b013e3182a28c2c

29. Solares CA, Strome M. Transoral robot-assisted CO2 laser supraglottic laryngectomy: experimental and clinical data. Laryngoscope (2007) 117(5):817-20. doi:10.1097/MLG.0b013e31803330b7

30. Cernea CR, Matos LL, de Carlucci Junior D, Leonhardt FD, Haddad L, Walder F. Transoral robotic supraglottic partial laryngectomy: report of the first Brazilian case. Braz JOtorhinolaryngol (2016). doi:10.1016/j.bjorl. 2016.01.016

31. Ansarin M, Zorzi S, Massaro MA, Tagliabue M, Proh M, Giugliano G, et al. Transoral robotic surgery vs transoral laser microsurgery for resection of supraglottic cancer: a pilot surgery. Int J Med Robot (2014) 10(1):107-12. doi: $10.1002 / \mathrm{rcs} .1546$ 
32. De Virgilio A, Park YM, Kim WS, Baek SJ, Kim SH. How to optimize laryngeal and hypopharyngeal exposure in transoral robotic surgery. Auris Nasus Larynx (2013) 40(3):312-9. doi:10.1016/j.anl.2012.07.017

33. Park YM, Byeon HK, Chung HP, Choi EC, Kim SH. Comparison of treatment outcomes after transoral robotic surgery and supraglottic partial laryngectomy: our experience with seventeen and seventeen patients respectively. Clin Otolaryngol (2013) 38(3):270-4. doi:10.1111/coa.12101

34. Dziegielewski PT, Kang SY, Ozer E. Transoral robotic surgery (TORS) for laryngeal and hypopharyngeal cancers. JSurg Oncol (2015) 112(7):702-6. doi:10.1002/jso.24002

35. Lawson G, Mendelsohn AH, Van Der Vorst S, Bachy V, Remacle M. Transoral robotic surgery total laryngectomy. Laryngoscope (2013) 123(1):193-6. doi:10.1002/lary.23287

36. Dowthwaite S, Nichols AC, Yoo J, Smith RV, Dhaliwal S, Basmaji J, et al. Transoral robotic total laryngectomy: report of 3 cases. Head Neck (2013) 35(11):E338-42. doi:10.1002/hed.23226

37. Smith RV, Schiff BA, Sarta C, Hans S, Brasnu D. Transoral robotic total laryngectomy. Laryngoscope (2013) 123(3):678-82. doi:10.1002/lary.23842

38. Krishnan G, Krishnan S. Transoral robotic surgery total laryngectomy: evaluation of functional and survival outcomes in a retrospective case series at a single institution. ORL J Otorhinolaryngol Relat Spec (2017) 79(4):191-201. doi:10.1159/000464138

39. Gorphe P, Von Tan J, El Bedoui S, Hartl DM, Auperin A, Qassemyar Q, et al. Early assessment of feasibility and technical specificities of transoral robotic surgery using the da Vinci Xi. J Robot Surg (2017) 11(4):455-61. doi:10.1007/ s11701-017-0679-z

40. Sjogren EV. Transoral laser microsurgery in early glottic lesions. Curr Otorhinolaryngol Rep (2017) 5(1):56-68. doi:10.1007/s40136-017-0148-2

41. Hartl DM, Ferlito A, Brasnu DF, Langendijk JA, Rinaldo A, Silver CE, et al. Evidence-based review of treatment options for patients with glottic cancer. Head Neck (2011) 33(11):1638-48. doi:10.1002/hed.21528

42. Peretti G, Piazza C, Mora F, Garofolo S, Guastini L. Reasonable limits for transoral laser microsurgery in laryngeal cancer. Curr Opin Otolaryngol Head Neck Surg (2016) 24(2):135-9. doi:10.1097/MOO.0000000000000240

43. O'Malley BW Jr, Weinstein GS, Hockstein NG. Transoral robotic surgery (TORS): glottic microsurgery in a canine model. J Voice (2006) 20(2):263-8. doi:10.1016/j.jvoice.2005.10.004

44. Park YM, Lee WJ, Lee JG, Lee WS, Choi EC, Chung SM, et al. Transoral robotic surgery (TORS) in laryngeal and hypopharyngeal cancer. J Laparoendosc $A d v$ Surg Tech A (2009) 19(3):361-8. doi:10.1089/lap.2008.0320

45. Blanco RG, Ha PK, Califano JA, Saunders JM. Transoral robotic surgery of the vocal cord. J Laparoendosc Adv Surg Tech A (2011) 21(2):157-9. doi:10.1089/ lap. 2010.0350
46. Vural E, Tulunay-Ugur OE, Suen JY. Transoral robotic supracricoid partial laryngectomy with cartilaginous framework preservation. J Robot Surg (2012) 6(4):363-6. doi:10.1007/s11701-012-0349-0

47. Kayhan FT, Kaya KH, Sayin I. Transoral robotic cordectomy for early glottic carcinoma. Ann Otol Rhinol Laryngol (2012) 121(8):497-502. doi:10.1177/000348941212100801

48. Wang CC, Liu SA, Wu SH, Lin WJ, Jiang RS, Wang L. Transoral robotic surgery for early glottic carcinoma involving anterior commissure: preliminary reports. Head Neck (2016) 38(6):913-8. doi:10.1002/hed.24354

49. Remacle M, Prasad VMN. Preliminary experience in transoral laryngeal surgery with a flexible robotic system for benign lesions of the vocal folds. Eur Arch Otorhinolaryngol (2018) 275(3):761-5. doi:10.1007/s00405-018-4900-0

50. Hans S, Badoual C, Gorphe P, Brasnu D. Transoral robotic surgery for head and neck carcinomas. Eur Arch Otorhinolaryngol (2012) 269(8):1979-84. doi:10.1007/s00405-011-1865-7

51. Mandapathil M, Greene B, Wilhelm T. Transoral surgery using a novel single-port flexible endoscope system. Eur Arch Otorhinolaryngol (2015) 272(9):2451-6. doi:10.1007/s00405-014-3177-1

52. Mandapathil M, Duvvuri U, Guldner C, Teymoortash A, Lawson G, Werner JA. Transoral surgery for oropharyngeal tumors using the Medrobotics((R)) Flex((R)) System-a case report. Int J Surg Case Rep (2015) 10:173-5. doi:10.1016/j.ijscr.2015.03.030

53. Remacle M, Prasad V, Lawson G, Plisson L, Bachy V, Van der Vorst S. Transoral robotic surgery (TORS) with the medrobotics flex system: first surgical application on humans. Eur Arch Otorhinolaryngol (2015) 272(6):1451-5. doi:10.1007/s00405-015-3532-x

54. Mattheis S, Hasskamp P, Holtmann L, Schafer C, Geisthoff U, Dominas N, et al. Flex robotic system in transoral robotic surgery: the first 40 patients. Head Neck (2017) 39(3):471-5. doi:10.1002/hed.24611

55. Lang S, Mattheis S, Hasskamp P, Lawson G, Guldner C, Mandapathil M, et al. A European multicenter study evaluating the flex robotic system in transoral robotic surgery. Laryngoscope (2017) 127(2):391-5. doi:10.1002/lary.26358

Conflict of Interest Statement: The author declares no conflict of interest. The article submitted did not involve any personal, professional, or financial relationships that could potentially be construed as a conflict of interest.

Copyright () 2018 Gorphe. This is an open-access article distributed under the terms of the Creative Commons Attribution License (CC BY). The use, distribution or reproduction in other forums is permitted, provided the original author(s) and the copyright owner are credited and that the original publication in this journal is cited, in accordance with accepted academic practice. No use, distribution or reproduction is permitted which does not comply with these terms. 


\section{OPEN ACCESS}

Edited by:

Vincent Vander Poorten KU-Leuven, Belgium

Reviewed by:

Davide Lombardi,

University of Brescia, Italy

Philippe Gorphe

Institut Gustave Roussy, France

*Correspondence:

Vanessa C. Stubbs

vanessa.stubbs@uphs.upenn.edu

Specialty section:

This article was submitted to

Head and Neck Cancer,

a section of the journal

Frontiers in Oncology

Received: 11 May 2018

Accepted: 23 July 2018

Published: 14 August 2018

Citation:

Stubbs VC, Rajasekaran K, Gigliotti AR, Mahmoud AF, Brody RM, Newman JG, Rassekh $\mathrm{CH}$ and Weinstein GS (2018) Management of the Airway for Transoral Robotic

Supraglottic Partial Laryngectomy. Front. Oncol. 8:312. doi: 10.3389/fonc.2018.00312

\section{Management of the Airway for Transoral Robotic Supraglottic Partial Laryngectomy}

\author{
Vanessa C. Stubbs ${ }^{1 *}$, Karthik Rajasekaran ${ }^{1}$, Adam R. Gigliotti ${ }^{1}$, Ahmad F. Mahmoud ${ }^{1}$, \\ Robert M. Brody ${ }^{2}$, Jason G. Newman ${ }^{1}$, Christopher H. Rassekh ${ }^{1}$ and \\ Gregory S. Weinstein ${ }^{1}$ \\ ${ }^{1}$ Department of Otorhinolaryngology: Head and Neck Surgery, University of Pennsylvania, Philadelphia, PA, United States, \\ ${ }^{2}$ Department of Otorhinolaryngology: Head and Neck Surgery, Medical University of South Carolina, Charleston, SC, \\ United States
}

Introduction: Over the last several decades, transoral resection techniques for treatment of supraglottic lesions have become increasingly favored to reduce the need for either open transcervical resection or primary chemoradiation. Transoral robotic surgery (TORS) offers advantages in visualization, dissection control, and access to remove bulky tumors en bloc. However, the management of the airway for these cases tends to vary, without clear guidelines as to when a tracheostomy is necessary.

Materials and Methods: A retrospective review of all patients who underwent transoral robotic supraglottic partial laryngectomy at a large academic center from May 2005 through December 2016 was performed. Airway management was examined, specifically as it pertains to whether a tracheostomy was performed at the time of surgery or otherwise. Demographic and tumor characteristics were also evaluated.

Results: Sixty-three patients were included. Forty (63\%) were male, the average age at surgery was 63.6, and the majority (90.5\%) underwent resection for squamous cell carcinoma of the supraglottis. Thirty-nine patients (62\%) underwent the procedure with standard endotracheal intubation using a wire-reinforced tube. Of these, four patients required subsequent tracheostomy- 2 for laryngeal edema postoperatively, one for airway management during a postoperative bleeding event, and one for laryngeal edema following initiation of adjuvant chemoradiation. Twenty patients (32\%) underwent tracheostomy at the time of transoral resection for airway management, 17 of whom were decannulated an average of 12.2 weeks following surgery. Those who underwent tracheostomy at the time of surgery had a higher percentage of tumors involving multiple supraglottic subsites $(p=0.031), 85$ vs. $54 \%$ in the group who did not undergo tracheostomy. No difference in age, BMI, clinical T-stage, or clinical N stage was found between the two groups.

Conclusion: Performing a tracheostomy at the time of surgery should be considered for those patients with more extensive malignant disease ( $\geq T 2$ tumors). While avoiding 
tracheostomy is often preferred by the patient, the maintenance of the patent airway perioperatively should be first priority when considering airway management. Furthermore, as the majority of those patients receiving tracheostomy are decannulated within 4 months of surgery, the tracheostomy could be considered a short-term adjunct to the procedure.

Keywords: transoral robotic surgery, supraglottic partial laryngectomy, organ preservation surgery, airway, tracheostomy

\section{INTRODUCTION}

Over the last few decades there have been several shifts in optimal treatment regimens for laryngeal cancer. While primary chemoradiation therapy has been utilized widely as an "organ preservation" option since the publication of the Veteran Affairs (VA) trial in 1991 to avoid disfigurement and morbidity associated with previous open surgical resections, this regimen has not been without its own toxicities $(1,2)$. With some series reporting a $24 \%$ incidence of persistent dysphagia, 10\% incidence of long term enteral access for feeding, and overall poor quality of life, there has been a movement toward minimally invasive transoral surgical techniques $(3,4)$. Both transoral laser microsurgery (TLM) and transoral robotic (TORS) partial supraglottic laryngectomy have been introduced as surgical organ preservation options. TLM has been shown to have both equivalent oncologic outcomes to open resection and chemoradiation as well as decreased morbidity, shorter hospital stays, and superior functional outcomes (5-7). Due to limitations of TLM including exposure, ability to obtain en bloc resection and difficulty in resection of large bulky tumors, TORS supraglottic partial laryngectomy was introduced as an alternative methodology by Weinstein et al. in 2007 following good feasibility outcomes utilizing TORS in resection of oropharyngeal malignancy (8-10). In several feasibility studies and institutional series published, TORS partial laryngectomy has been considered a safe and oncologically sound treatment option (11-16). However, there is a lack of data in these studies regarding airway management and need for tracheostomy. Traditionally, tracheostomy has been indicated for several reasons in this setting: to provide exposure and additional operative space during surgery in patients with bulky tumors, to maintain a patent airway in those with postoperative laryngeal edema, and to protect the lower airway in the case of bleeding event postoperatively. In this study, we aim to evaluate the airway management technique in this institution's series of patients undergoing TORS supraglottic partial laryngectomy.

\section{MATERIALS AND METHODS}

A retrospective review of all patients who underwent transoral robotic supraglottic partial laryngectomy at a large academic medical center from May 2005 through December 2016 was performed. Institutional Review Board approval was obtained for this retrospective study from the University of Pennsylvania

Abbreviations: TORS, transoral robotic surgery; TLM, transoral laser microsurgery; BMI, body mass index; SD, Standard Deviation.
Office of Clinical Research, and all subjects gave written informed consent. The electronic medical record system as well as archived paper medical records were utilized to collect patient demographic information including age, sex, weight, body mass index (BMI), past medical history and active medications, specifically anticoagulation/antiplatelet therapies, at the time of surgery; tumor information including tissue diagnosis, primary disease subsite within the supraglottic, involvement of multiple supraglottic subsites, TNM stage, pre-epiglottic, and paraglottic space involvement; and treatment information including margin status, management of the nodal disease, need for neck dissection, and need for adjuvant therapy.

Airway management information was also obtained including tracheostomy placement timing, date of decannulation if applicable, long term tracheostomy tube dependence, and reason for delayed tracheostomy placement if applicable.

The transoral robotic supraglottic partial laryngectomy procedure is a standardized procedure at our institution with bilateral supraglottic resection performed routinely for all tumors.

Neck dissection was the primary treatment for management of the neck in this patient cohort as determined by the surgeon in conjunction with the multidisciplinary tumor board. Adjuvant radiotherapy with or without chemotherapy was recommended for patients with pathology results indicating close or positive surgical margins for a tumor at the primary site, involvement of 2 or more lymph nodes, perineural or lymphovascular invasion, or extracapsular spread of nodal metastasis.

Two sample $t$-testing, two-sample test of proportions, twosample Wilcoxon rank sum (Mann-Whitney), and Fisher exact testing was used to compare differences in characteristics between patients who underwent tracheostomy at the time of TORS and those who did not undergo tracheostomy. Multivariate regression analysis was used in order to assess the relationship between time to decannulation with adjuvant radiation and chemotherapy. Statistical analysis was performed using Stata (Stata Statistical Software: Release 15. College Station, TX: StataCorp LLC).

\section{RESULTS}

Sixty-three patients underwent transoral robotic partial laryngectomy at our institution between May 2005 and December 2016. There was a male predominance (63\%), an average age at surgery of 63.6 years (range [20,84], $S D=10.4$ ) and an average follow-up time of 49.7 months (range $[2.4,126.9]$, $S D=34.8)$. The majority of patients $(91 \%)$ underwent resection 
for squamous cell carcinoma. The other pathologies are listed in Table 1. The majority of lesions were primarily involving of the epiglottis $(62.0 \%)$, followed by the aryepiglottic fold (14.3\%), the arytenoid (6.3\%), and the false cord (1.2\%). Forty patients presented with multiple subsites involved (63.5\%). Patients included with epithelial carcinomas had T1-T3 tumors, majority with T2 tumors (44.8\%). Tumor data was unable to be obtained for five patients.

There were no intraoperative complications (0\%). Two patients experienced temporary postoperative complications: one patient experienced postoperative bleeding requiring surgical intervention, and one patient experienced a myocardial infarction for which he underwent subsequent coronary bypass surgery. Length of hospitalization ranged from 2 to 37 days (median $=5, S D=6.3$ ).

Neck dissection was indicated and performed in $74.6 \%$ of patients, the majority of which were bilateral (78.7\%). Timing of the neck dissection with regards to the TORS supraglottic partial laryngectomy resection varied: $74.5 \%$ of patients underwent neck dissection following TORS procedure (at a mean of 39 days following the procedure), $23.4 \%$ underwent neck dissection prior to TORS resection, and $2.1 \%$ had neck dissection performed at the time of the TORS resection. Thirty-two percent of patients underwent adjuvant radiotherapy and 25\% underwent adjuvant chemotherapy.

With regards to airway management, 39 patients $(62 \%)$ underwent the procedure with standard endotracheal intubation using a wire-reinforced tube and did not undergo tracheostomy at the time of the supraglottic partial laryngectomy. Of these, 4 patients required subsequent tracheostomy. One of these patients underwent tracheostomy for airway management during a second bleeding event post-operatively. This patient had previously been extubated without difficulty twice prior to placement of the tracheostomy tube 13 days following their initial resection and was subsequently successfully decannulated 5 weeks following without complication. Two patients underwent tracheostomy at 3 and 6 weeks following the TORS procedure due to laryngeal edema. The last patient underwent delayed tracheostomy (4 months after surgery) due to laryngeal edema following initiation of adjuvant chemoradiation.

Twenty patients (32\%) underwent tracheostomy at the time of transoral resection for airway management, 17 of whom were subsequently decannulated an average of 12.2 weeks following surgery. From this group, 11 patients were decannulated an average of 38 days following surgery, six others had tracheostomy for more than 3 months (range 145-243 days), and 3 patients

TABLE 1 | Disease pathology of all included patients.

\begin{tabular}{lc}
\hline Pathology & $\mathbf{N}(\%)$ \\
\hline Squamous cell carcinoma & $57(90.5 \%)$ \\
Paraganglioma & $2(3.2 \%)$ \\
Adenosquamous carcinoma & $1(1.6 \%)$ \\
Chondrosarcoma & $1(1.6 \%)$ \\
Mucoepidermoid carcinoma (low grade) & $1(1.6 \%)$ \\
Amyloidosis & $1(1.6 \%)$
\end{tabular}

remained with a permanent tracheostomy. There were no airway complications postoperatively in this group of patients. An additional four patients who underwent the TORS supraglottic partial laryngectomy procedure had previously undergone tracheostomy prior to their procedure. Two of these patients had required tracheostomy on an emergent basis due to airway obstruction from their laryngeal tumor prior to consideration for surgical resection, while one patient had concomitant tonsillar squamous cell carcinoma and underwent tracheostomy previously. The final patient had recurrent chondrosarcoma of the arytenoid cartilage and had a long-standing tracheostomy prior to their procedure. All patients with prior tracheostomy were excluded from analysis regarding need for tracheostomy at the time of surgery.

Those who underwent tracheostomy at the time of surgery had no difference in age, sex, BMI, clinical T-stage, clinical $\mathrm{N}$ stage, or length of stay compared to those who did not undergo tracheostomy. However, there was a significant difference between the two groups when looking at tumor involvement of multiple subsites. Fifty-four percent of patients who did not undergo tracheostomy had tumors that involved multiple subsites, compared to $85 \%$ of patients who underwent tracheostomy $(p=0.031,95 \%$ CI $[-0.51,-0.06])$. Patient characteristics of each group can be found in Table 2 . There was also no difference in active use of anticoagulation or antiplatelet therapies (including warfarin, factor XA inhibitors such as rivaroxaban, clopidogrel, and full dose aspirin) between the two groups. Furthermore, no difference was found in history of pulmonary comorbidity.

In those who underwent tracheostomy at the time of TORS resection, $58.8 \%$ of patients had neck dissection following their surgery while $41.2 \%$ of patient had neck dissection performed previously. In the patients that did not receive tracheostomy, $82.8 \%$ of patients underwent delayed neck dissection while $13.8 \%$ of patients had neck dissections preformed previously and $3.4 \%$ had neck dissection at the time of TORS. While, a greater proportion of patients who did not receive tracheostomy had delayed neck dissections, this only trended toward significance $(p=0.070)$. Neck dissection data can be seen in Table 2.

For the patients who underwent tracheostomy during treatment, there was a significant difference in time to decannulation with regards to adjuvant therapy. For those who received adjuvant radiotherapy, there was a positive correlation with time to decannulation, with the average time to decannulation equal to 16 weeks compared to 14 weeks for those who did not receive radiation therapy $(p=0.039)$. Adjuvant chemotherapy had a negative correlation with time to decannulation with the average time to decannulation at 10 weeks compared to 17 weeks for those who did receive chemotherapy $(p=0.023)$.

A trend toward tracheostomy at the time of surgery was found over the course of the study period (Figure 1). From the years 2005 through 2008, 22 patients underwent TORS supraglottic partial laryngectomy and only 1 patient (4.5\%) underwent tracheostomy at the time of surgery. Of the 20 patients who underwent the procedure from 2009 through 2012, 10 patients (50\%) underwent tracheostomy at the time of surgery. From 2013 
TABLE 2 | Characteristics of patients who underwent tracheostomy at the time of surgery compared to those in which tracheostomy was not performed at the time of resection.

\begin{tabular}{|c|c|c|c|}
\hline Characteristic & Tracheostomy at time of TORS & No Tracheostomy & $P$ value \\
\hline$N$ & 20 & 39 & \\
\hline Age (mean, $S D$ ) & $67.0(8.4)$ & $62.3(11.3)$ & 0.087 \\
\hline Male & $14(70.0 \%)$ & $23(59.0 \%)$ & \\
\hline Female & $6(30.0 \%)$ & $16(41.0 \%)$ & \\
\hline Weight (kg) & 76.4 & 74.2 & 0.590 \\
\hline Active anticoagulation at time of surgery & $4(20.0 \%)$ & $5(12.8 \%)$ & 0.416 \\
\hline Warfarin & 1 & 1 & \\
\hline Clopidogrel & 2 & 3 & \\
\hline Aspirin (full strength) & 1 & 1 & \\
\hline Squamous cell carcinoma pathology & $20(100 \%)$ & 35 (89.7\%) & \\
\hline False vocal cord & $0(0 \%)$ & $1(2.6 \%)$ & \\
\hline Multiple subsites involved & 17 (85.0\%) & $21(53.8 \%)$ & 0.031 \\
\hline T Stage & & & 0.212 \\
\hline $\mathrm{T} 1$ & 1 & 8 & \\
\hline T2 & 12 & 14 & \\
\hline Т3 & 4 & 6 & \\
\hline Recurrence & 3 & 3 & \\
\hline Unknown & 0 & 5 & \\
\hline N/A (non-epithelial) & 0 & 3 & \\
\hline N Stage & & & 0.389 \\
\hline NO & 17 & 24 & \\
\hline $\mathrm{N} 1$ & 0 & 3 & \\
\hline Not performed & 3 & 10 & \\
\hline Timing of Neck Dissection & & & 0.070 \\
\hline Prior to TORS (\% of neck dissections performed) & $7(41.2 \%)$ & $4(13.8 \%)$ & \\
\hline Concurrent with TORS (\% of neck dissections performed) & $0(0.0 \%)$ & $1(3.4 \%)$ & \\
\hline After TORS (\% of neck dissections performed) & $10(58.8 \%)$ & $24(82.8 \%)$ & \\
\hline Not Performed & 3 & 10 & \\
\hline
\end{tabular}

Statistically significant values with $P<0.05$ in bold.

through 2016, 9 patients (52.9\%) underwent tracheostomy at the time of TORS procedure out of a total of 17 patients. A similar trend in tumor size was not found during this time period. From 2005 through 2008, 21\% of patients presented with T1 disease, with $79 \%$ having $>=\mathrm{T} 2$ disease. While only $6 \%$ of patients from 2009 through 2012 had T1 disease (94\% with >=T2 disease), $31 \%$ presented with $\mathrm{T} 1$ disease ( $69 \%$ with $>=\mathrm{T} 2$ disease) during the years 2013 through 2016.

\section{DISCUSSION}

In this retrospective study, we examined airway management, specifically need for tracheostomy, during TORS supraglottic partial laryngectomy over a 16-year period. While several previously published institutional studies have commented on airway management, they have primarily focused on the feasibility of performing the procedure rather than on how the airway was managed. Mendelsohn et al. published their experience with TORS supraglottic partial laryngectomy in 2013. The study examined the cases of 18 patients, all of whom completed the procedure with orotracheal intubation and were kept intubated until post-operative day one (11). Similarly, in their series of 13 patients in 2013, Ozer et al. noted that no patient underwent tracheostomy at the time of surgery and all patients were extubated immediately post-operatively; however, one patient required delayed tracheostomy (15). Park et al. also 


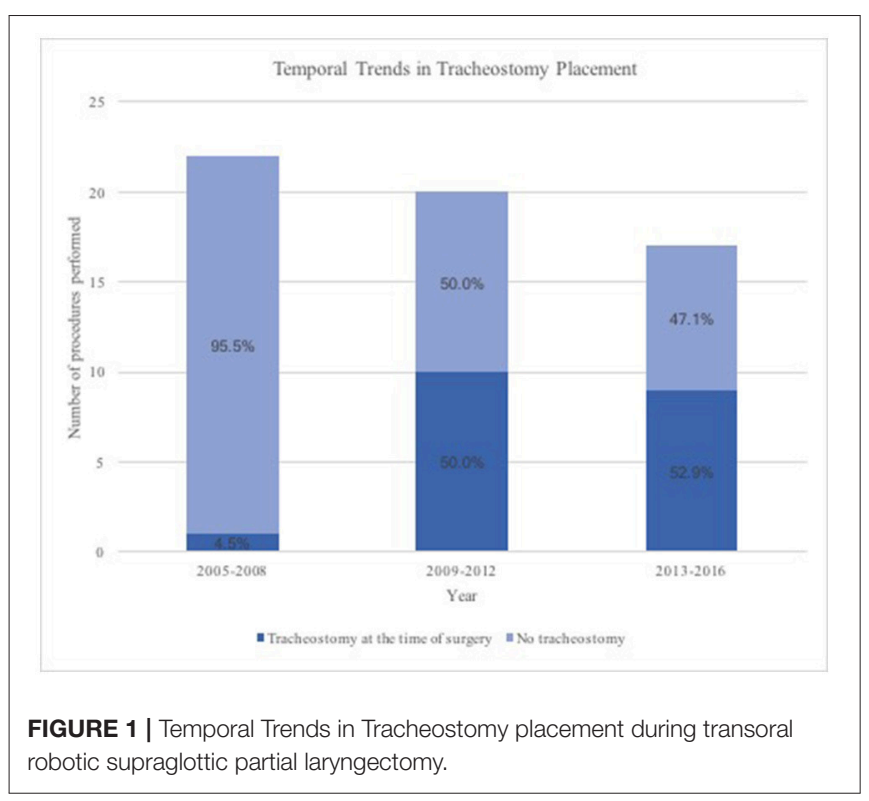

published a feasibility study in which 16 patients were included, all of whom underwent temporary tracheostomy at the time of surgery with a mean time of decannulation of 11 days (12). Given that the dataset in this study examines both cases in which the airway was managed by both intubation only and also tracheostomy at the time of surgery, the outcomes of each could be evaluated.

Furthermore in 2015, Razafindranaly et al. published a multicenter retrospective study examining outcomes following transoral robotic supraglottic partial laryngectomy including 84 patients (16). Of these, 20 patients (24\%) underwent tracheostomy, 12 of whom underwent simultaneous tracheostomy during the time of surgery due to high risk for laryngeal edema postoperatively and 8 who underwent tracheostomy following resection due to dyspnea postoperatively. The overall tracheostomy rate of $24 \%$ of this study was slightly less than what we found in the present study during which $38 \%$ underwent tracheostomy; however, a larger proportion of those receiving tracheostomy in our study underwent tracheostomy during the resection as compared to post-operatively in the setting of dyspnea (83 vs. 60\%). As the patient $\mathrm{T}$ stages were similar between the two studies with majority being T2 lesions, this may reflect a lower threshold of our institution to prophylactically perform tracheostomy. Additionally, while the previous study discusses perioperative airway management, it did not mention which characteristics were taken into account in determining risk for postoperative laryngeal edema. The aim of the current study is to expand on those characteristics which may put patients at higher risk for need for tracheostomy.

Tumor involvement of multiple subsites was noted to be significantly different between the two groups, with an increased percentage of multiple subsite involvement in the tracheostomy group. Although no significant difference was found in clinical T stage as a whole between the groups, there was a difference when looking at proportions of $\mathrm{T} 1$ compared to $\geq \mathrm{T} 2$ tumors. This finding suggests that the presence of a tumor involving multiple subsites ( $\geq \mathrm{T} 2$ ) could be a factor in consideration of tracheostomy at the time of surgery. Given that the extent of resection during transoral supraglottic partial laryngectomy at our institution is standardized, ability to gain exposure and additional operative space was a factor in decision for tracheostomy for bulkier $\geq \mathrm{T} 2$ tumors and likely contributes to this finding.

It was also noted that neck dissection was more likely to be delayed in those patients who did not undergo tracheostomy at the time of TORS resection, though this only trended toward significance. Bilateral neck dissection is indicated in the majority of patients with supraglottic malignancy and carries the possibility of increased laryngeal edema if performed simultaneously with laryngeal resection. Therefore, this should be taken into account when considering airway management. In those patients without other risk factors undergoing the TORS procedure without tracheostomy, consideration should be given to staging the neck dissection.

While we found no difference in age, BMI, pulmonary comorbidities, active anticoagulation/antiplatelet medications, clinical T stage, clinical N stage, or length of stay between those who underwent tracheostomy at the time of surgery and those who did not, it should be noted that all patients who underwent tracheostomy had diagnoses of squamous cell carcinoma as opposed to other benign pathology. For each patient in this study, a priority was made to evaluate the optimal airway management for each patient on a case by case basis with emphasis placed on difficulty of exposure during intubation, extent of disease resection, and body habitus of the patient that may prevent easy reintubation.

For those who underwent tracheostomy, we found that time to decannulation was increased for those who underwent adjuvant radiotherapy. This correlation is likely due to acute side effects of radiation therapy including laryngeal edema. As $45 \%$ of the patients who underwent tracheostomy also underwent adjuvant radiotherapy, it is possible that this also increased the average time to decannulation that was longer in our study as compared to previous studies (12.2 weeks vs. 11 days in the study by Park et al.) (12). At our institution, a conservative approach was taken with regards to decannulation in order to avoid possible need for repeat tracheostomy at the time of adjuvant therapy when the procedure would have the potential to be more complicated given a previously operated neck or the possibility of requirement of urgent or emergent tracheostomy in a less controlled setting.

We also found a temporal trend at our institution toward tracheostomy over time. When looking at the same time periods, there was not a corresponding trend in tumor size over time that could explain this shift. This may be due to changing attitudes toward risk of airway complications postoperatively. While avoiding tracheostomy is often preferred by the patient, the maintenance of the patent airway peri-operatively should be first priority when considering airway management. Furthermore, it should be noted that the majority of those patients receiving tracheostomy were decannulated within 4 months of surgery. Therefore, the tracheostomy could be considered a short-term adjunct to the procedure. 
This current study is limited by its retrospective nature. Although patient demographics and tumor characteristics were the main areas of examination, other factors that could not be corrected for may have played a part in airway decision making. Furthermore, given the temporal changes in practice over time, it is likely that surgeon preferences may also have contributed to changes in management. Although this study includes one of the largest published patient series, it nevertheless is still limited by sample size. Future large prospective studies would be needed to limit these biases; however, randomized trials would be difficult given patient strong preference against tracheostomy.

\section{CONCLUSION}

While previous studies have examined the feasibility of performing TORS supraglottic partial laryngectomy, less emphasis has been placed on perioperative airway management. In the present study, we examined the management technique of the airway, whether tracheostomy was performed either at the time of surgery or otherwise, in 63 patients who underwent the procedure. A higher proportion of patients who underwent tracheostomy at the time of surgery had multiple subsite involvement of their tumors than those who underwent routine orotracheal intubation without tracheostomy placement. Those who did not undergo tracheostomy tended to have a staged neck dissection that could potentially limit laryngeal edema at the

\section{REFERENCES}

1. Hoffman HT, Porter K, Karnell LH, Cooper JS, Weber RS, Langer CJ, et al. Laryngeal cancer in the United States: changes in demographics, patterns of care, and survival. Larygoscope (2006) 116(9 Pt. 2 Suppl. 111):1-13. doi: 10.1097/01.mlg.0000236095.97947.26

2. Chen AY, Fedewa S, Zhu J. Temporal trends in the treatment of earlyand advanced-stage laryngeal cancer in the united states, 1985-2007. Arch Otolaryngol Neck Surg. (2011) 137:1017-24. doi: 10.1001/archoto.2011.171

3. Sessions DG, Lenox J, Spector GJ. Supraglottic laryngeal cancer: analysis of treatment results. Laryngoscope (2005) 115:1402-10. doi: 10.1097/01.MLG.0000166896.67924.B7

4. Hanna E, Sherman A, Cash D, Adams D, Vural E, Fan CY, et al. Quality of life for patients following total laryngectomy vs chemoradiation for laryngeal preservation. Arch Otolaryngol Neck Surg. (2004) 130:875-9. doi: 10.1001/archotol.130.7.875

5. Grant DG, Salassa JR, Hinni ML, Pearson BW,Hayden RE, Perry WC. Transoral laser microsurgery for carcinoma of the supraglottic larynx. Otolaryngol Neck Surg. (2007) 136:900-6. doi: 10.1016/j.otohns.2006.1 2.015

6. Cabanillas R, Rodrigo JP, Llorente JL, Suárez C. Oncologic outcomes of transoral laser surgery of supraglottic carcinoma compared with a transcervical approach. Head Neck (2008) 30:750-5. doi: 10.1002/hed.20778

7. Peretti G, Piazza C, Cattaneo A, De Benedetto L, Martin E, Nicolai P. Comparison of functional outcomes after endoscopic versus open-neck supraglottic laryngectomies. Ann Otol Rhinol Laryngol. (2006) 115:827-32. doi: $10.1177 / 000348940611501106$

8. Weinstein GS, O'Malley BWJ, Snyder W, Hockstein NG. Transoral robotic surgery: supraglottic partial laryngectomy. Ann Otol Rhinol Laryngol. (2007) 116:19-23. doi: 10.1177/000348940711600104

9. Weinstein GS, O'Malley BW, Jr, Snyder W, Sherman E, Quon H. Transoral robotic surgery: radical tonsillectomy. Arch Otolaryngol Neck Surg. (2007) 133:1220-6. doi: 10.1001/archotol.133.1 2.1220 time of resection. Although we found no difference in age, BMI, pulmonary comorbidities, active anticoagulation/antiplatelet medications, or clinical $\mathrm{N}$ stage between the two groups, we did find a temporal trend toward tracheostomy at the time of surgery. This data supports the idea that each patient case should be evaluated individually, but also that tumor involvement of multiple supraglottic subsites ( $\geq \mathrm{T} 2$ tumors) may help to guide need for tracheostomy. While avoiding tracheostomy is often preferred by the patient, the maintenance of the patent airway perioperatively should be first priority when considering airway management. Ability to gain adequate access to the tumor for a complete oncologic resection may also play a part in decision making for tracheostomy. Given the results of this study, our institution is inclined to have a low threshold for temporary tracheostomy. If tracheostomy is performed, it is considered temporary and may prevent life threatening airway emergencies in the immediate postoperative period.

\section{AUTHOR CONTRIBUTIONS}

VS: study design, preparation of manuscript, data collection, interpretation of data, and revision of manuscript. KR: study design, interpretation of data, and preparation of manuscript. AG, RB: study design and data collection. AM: study design, statistical analysis, and interpretation of data. JN, CR, GW: study design, interpretation of data, and revision of manuscript.

10. O'Malley BW, Weinstein GS, Snyder W, Hockstein NG. Transoral Robotic Surgery (TORS) for base of tongue neoplasms. Laryngoscope (2006) 116:146572. doi: $10.1097 / 01 . \mathrm{mlg} .0000227184 .90514 .1 \mathrm{a}$

11. Mendelsohn AH, Remacle M, Van Der Vorst S, Bachy V, Lawson G. Outcomes following transoral robotic surgery: Supraglottic laryngectomy. Laryngoscope. (2013) 123:208-14. doi: 10.1002/lary.23621

12. Park YM, Lee WJ, Lee JG, Lee WS, Choi EC, Chung SM, et al. Transoral Robotic Surgery (TORS) in Laryngeal and Hypopharyngeal Cancer. $J$ Laparoendosc Adv Surg Tech. (2009) 19:361-8. doi: 10.1089/lap.2008.0320

13. Olsen SM, Moore EJ, Koch CA, Price DL, Kasperbauer JL, Olsen KD. Transoral robotic surgery for supraglottic squamous cell carcinoma. Am J Otolaryngol. (2012) 33:379-84. doi: 10.1016/j.amjoto.2011.10.007

14. Kayhan FT, Kaya KH, Altintas A, Sayin I. Transoral robotic supraglottic partial laryngectomy. J Craniofac Surg. (2014) 25:1422-6. doi: $10.1097 /$ SCS.0000000000000572

15. Ozer E, Alvarez B, Kakarala K, Durmus K, Teknos TN, Carrau RL. Clinical outcomes of transoral robotic supraglottic laryngectomy. Head Amp Neck (2013) 35:1158-61. doi: 10.1002/hed.23101

16. Razafindranaly V, Lallemant B, Aubry K, Moriniere S, Vergez S, Mones ED, et al. Clinical outcomes with transoral robotic surgery for supraglottic squamous cell carcinoma: Experience of a French evaluation cooperative subgroup of GETTEC. Head Neck (2015) 38:E1097-101. doi: 10.1002/hed.24163

Conflict of Interest Statement: The authors declare that the research was conducted in the absence of any commercial or financial relationships that could be construed as a potential conflict of interest.

Copyright (๑) 2018 Stubbs, Rajasekaran, Gigliotti, Mahmoud, Brody, Newman, Rassekh and Weinstein. This is an open-access article distributed under the terms of the Creative Commons Attribution License (CC BY). The use, distribution or reproduction in other forums is permitted, provided the original author(s) and the copyright owner(s) are credited and that the original publication in this journal is cited, in accordance with accepted academic practice. No use, distribution or reproduction is permitted which does not comply with these terms. 


\section{Transoral Robotic Surgery in the Nordic Countries: Current Status and Perspectives}

\begin{abstract}
Antti A. Mäkitie ${ }^{1,2 \star}$, Harri Keski-Säntti ${ }^{1}$, Mari Markkanen-Leppänen ${ }^{1}$, Leif Bäck ${ }^{1}$, Petri Koivunen ${ }^{3}$, Tomas Ekberg ${ }^{4}$, Karl Sandström ${ }^{4}$, Göran Laurell ${ }^{4}$, Mathias von Beckerath ${ }^{5}$, Johan S. Nilsson ${ }^{6}$, Peter Wahlberg ${ }^{6}$, Lennart Greiff, Lena Norberg Spaak ${ }^{7}$, Thomas Kjærgaard ${ }^{8}$, Christian Godballe ${ }^{9}$, Oddveig Rikardsen ${ }^{10}$, Hani Ibrahim Channir ${ }^{11}$, Niclas Rubek ${ }^{11}$ and Christian von Buchwald ${ }^{11}$

${ }^{1}$ Department of Otorhinolaryngology - Head and Neck Surgery, University of Helsinki and Helsinki University Hospital, Helsinki, Finland, ${ }^{2}$ Division of Ear, Nose and Throat Diseases, Department of Clinical Sciences, Intervention and Technology, Karolinska Institutet and Karolinska University Hospital, Stockholm, Sweden, ${ }^{3}$ Department of Otorhinolaryngology - Head and Neck Surgery, Oulu University Hospital, Oulu, Finland, ${ }^{4}$ Department of Surgical Sciences, Uppsala University, Uppsala, Sweden, ${ }^{5}$ Department of Otorhinolaryngology - Head and Neck Surgery, Örebro University Hospital, Örebro, Sweden, ${ }^{6}$ Department of Otorhinolaryngology - Head and Neck Surgery, Skåne University Hospital, Lund, Sweden, ${ }^{7}$ Department of Otorhinolaryngology - Head and Neck Surgery, Umeå University Hospital, Umeå, Sweden, ${ }^{8}$ Department of Otorhinolaryngology - Head and Neck Surgery, Aarhus University Hospital, Aarhus, Denmark, ${ }^{9}$ Department of Otorhinolaryngology - Head and Neck Surgery, Odense University Hospital, Odense, Denmark, ${ }^{10}$ Department of Otorhinolaryngology - Head and Neck Surgery, University Hospital North Norway, Tromsoe, Norway, ${ }^{11}$ Department of Otorhinolaryngology, Head and Neck Surgery and Audiology, Rigshospitalet, University of Copenhagen, Copenhagen, Denmark
\end{abstract}

Reviewed by:

Giuseppe Spriano, Department of Otolaryngology Head and Neck Surgery National Cancer

Institute, Italy Christopher H. Rassekh, University of Pennsylvania, United States

${ }^{*}$ Correspondence: Antti A. Mäkitie antti.makitie@helsinki.fi

Specialty section

This article was submitted to Head and Neck Cancer, a section of the journal Frontiers in Oncology

Received: 01 March 2018 Accepted: 09 July 2018 Published: 27 July 2018

Citation: Mäkitie AA, Keski-Säntti $H$ Markkanen-Leppänen M, Bäck L, Koivunen P, Ekberg T, Sandström K, Laurell $G$, von Beckerath $M$, Nilsson JS, Wahlberg P, Greiff L, Norberg Spaak L, Kjærgaard T, Godballe C

Rikardsen O, Channir HI, Rubek N and von Buchwald C (2018) Transoral Robotic Surgery in the Nordic Countries: Current Status and

Perspectives. Front. Oncol. 8:289. doi: 10.3389/fonc. 2018.00289
Background: The five Nordic countries with a population of $27 \mathrm{M}$ people form a rather homogenous region in terms of health care. The management of head and neck cancer is centralized to the 21 university hospitals in these countries. Our aim was to gain an overview of the volume and role of transoral robotic surgery (TORS) and to evaluate the need to centralize it in this area as the field is rapidly developing.

Materials and Methods: A structured questionnaire was sent to all 10 Departments of Otorhinolaryngology - Head and Neck Surgery in the Nordic countries having an active programme for TORS in December 2017.

Results: The total cumulative number of performed robotic surgeries at these 10 Nordic centers was 528 and varied between 5 and 240 per center. The median annual number of robotic surgeries was 38 (range, 5-60). The observed number of annually operated cases remained fairly low $(<25)$ at most of the centers.

Conclusions: The present results showing a limited volume of performed surgeries call for considerations to further centralize TORS in the Nordic countries.

Keywords: head and neck, surgery, management, centralization, cancer

\section{INTRODUCTION}

The five Nordic countries (Denmark, Finland, Iceland, Norway, and Sweden) with a population of $27 \mathrm{M}$ people form a rather homogenous region in terms of health care. The planning and the treatment for head and neck cancer (HNC) for all patients is centralized to the 21 university hospitals only (1). It is regulated by governmental authorities and organized by the public health care system in a fairly unified manner in all of the countries. 
Robotic surgery forms an interesting approach in HNC management, but the technical setting has so far warranted a remarkable financial investment, which has obviously resulted in a limited popularity and shared use of existing robots between several surgical specialties at each institution. Since its first use in transoral surgery in 2005 only a limited number of HNC centers worldwide have so far adopted this treatment modality $(2,3)$. However, along with the increasing incidence of HPV-related oropharyngeal squamous cell cancer (OPSCC) in the Western world the robotic technology continues to develop, and its indications seem to expand to new areas in the management head and neck tumors. Therefore, many Departments of Otorhinolaryngology-Head and Neck Surgery (ORL - HNS) consider including TORS as an option in their surgical armamentarium.

We performed a survey in the Nordic countries aiming to evaluate the current use of transoral robotic surgery (TORS) at the Departments of ORL - HNS. The results of the study might offer tools for initiatives to centralize this treatment modality or to even consider prospective multicenter studies in order to implement evidence-based TORS.

\section{MATERIALS AND METHODS}

TORS is performed at three (Copenhagen, Århus, Odense) out of the four university hospitals, which manage the HNC treatment in Denmark (5.7 M), at two (Helsinki and Oulu) out of the five in Finland $(5.5 \mathrm{M})$, at one (Tromsoe) out of the four in Norway (5.2 M), and at four (Lund, Örebro, Umeå, Uppsala) out of the seven in Sweden $(10 \mathrm{M})$. Iceland has not yet initiated a TORS programme. The number of inhabitants in these referral areas varies from 0.5 to $2.1 \mathrm{M}$ (total population $27 \mathrm{M}$ ).

A structured questionnaire was sent in December 2017 to all 10 Nordic Departments ORL - HNS performing TORS. The following data were recorded: the number of inhabitants in the referral area as well as patients referred for TORS from abroad, date of the start of robotic surgery, number of robots and their model, number of surgeons performing TORS, other specialties using the same robot, availability of the robot, surgical indications, and number of performed surgeries specified for each anatomical subsite.

\section{RESULTS}

The Department of ORL-HNS at the Lund University Hospital has used robotic surgery since 2008. The hospital currently has four robots available, whereas all the other centers have started TORS after 2013 and have only one robot dedicated for their use. Solely the Department of ORL-HNS in Copenhagen has their own robot in contrast to all the other centers, which share operative time slots with one to seven other surgical specialties. Half of the reported centers reported insufficient time slots to utilize the robot. All centers currently use the Da Vinci Si model except Aarhus and Odense University Hospitals, which are using the Xi model. The number of trained robotic surgeons for TORS varies from one to three at each Head and Neck center.
The total cumulative number of performed robotic surgeries by the end of 2017 at the 10 centers was 528 and varied between 5 and 240 per center. The median annual number of robotic surgeries was 38 (range, 5-60). The main indication for TORS was the treatment of various neoplasms $(N=423)$. The remaining indications consisted of diagnostic lingual tonsil resections in cases with head and neck carcinoma with unknown primary (HN-CUP), various benign lesions, and of tonsillectomies for training purposes in the initial learning phase of TORS. One center in Sweden and one in Denmark reported having operated on TORS patients also from abroad.

Table 1 shows the distribution of anatomical subsites for tumor resections in this series. The most frequent resection site was base of the tongue (53\%). There were no reported TORS operations performed for laryngeal glottic tumors.

\section{DISCUSSION}

In this study we performed a survey on the performance of robotic head and neck surgery at the Departments of ORLHNS in the five Nordic countries to be able to consider the need and possibilities to centralize this treatment modality in this area with a total of $27 \mathrm{M}$ people. Therefore, it remains obvious that although the technology has been available for more than 10 years, there are currently only 10 centers out of the 21 Departments of ORL-HNS in the Nordic countries that utilize TORS. Furthermore, it is noteworthy, that in the Baltic countries (Estonia, Latvia, Lithuania; total $6 \mathrm{M}$ people) there are currently no surgical robots available. The present study thus covers the whole Northern Europe. However, along with the increasing numbers of OPSCC in the Western world and the improved technical solutions and new surgical indications, robotic surgery may be popularized even more among the institutions managing HNC.

Chen et al. concluded from their National Cancer Database data $(n=877)$ for adults with OPSCC who had undergone TORS, that high-volume centers have the lowest rates of positive margins and unplanned readmissions (4). Similar results were reported by Cracchiolo et al. (5) in a series of 846 OPSCC cases undergoing TORS: positive margin rates were lower when TORS was performed at a high volume vs. low volume hospital (8.2 vs. $16.7 \%$ respectively, $p=0.001)$ (5). It has been

TABLE 1 | Distribution of the anatomical subsites for tumor resections in the series of 484 TORS cases in the Nordic countries during the 10-year period between 2008 and 2017.

\begin{tabular}{lc}
\hline Subsite & Number (\%) of surgeries \\
\hline Base of tongue & $255(53)$ \\
Other oropharyngeal & $179(37)$ \\
Nasopharynx & $2(0.4)$ \\
Hypopharynx & $11(2)$ \\
Larynx & \\
Supraglottic & $37(8)$ \\
Glottic & 0 \\
\hline
\end{tabular}

*The number of base of tongue resections includes the lingual tonsil resections performed as part of the HN-CUP diagnostic work up. 
suggested that consolidating TORS procedures of early stage OPSCC to create high-volume centers of excellence might be a potential strategy to increase incremental effectiveness and reduce incremental costs (6). For other surgical fields there are reports advocating for the centralization of robotic surgery in order to establish high-volume centers with better training possibilities and consequently more efficient use of operating time and thus reduced costs $(7-10)$. The results of the present survey call for considerations to centralize TORS in the Nordic countries. The observed number of annually operated cases (median 38) was fairly low $(<25)$ at most of the centers i.e., at nine out of the ten university hospitals. This result is obviously slightly biased since two of the ten centers had started their TORS activities shortly before the survey and could thus not report their annual number of operated cases. Both of these centers (Odense and Umeå) have now operated 2-4 patients per month during the past weeks, which will eventually affect the median number of annually operated TORS cases in these areas. At the time of the survey in December 2017 only the Copenhagen University Hospital reported one TORS case performed on average each week (total number of 240 cases since 2013) (11). Furthermore, in Iceland, the Department of ORL-HNS does not utilize a robot and in Norway, only one center (Tromsoe) has adopted this technology in 2016 and has now the experience of 37 surgeries.

Since the introduction of TORS more than a decade ago, the indications for robotic surgery in the head and neck area have expanded from including oropharyngeal tumors to neoplasms of the nasopharynx, hypopharynx, larynx and thyroid, and most recently also skull base and neck dissection. The typical anatomical subsite for the primary tumor in the present series was either in the palatine or lingual tonsils, which are easily accessible with TORS. Half of the TORS procedures involved the base of the tongue, which has been shown also by others (11). This is the result of the increasing number of HNC cases with HPV-related HN-CUP and the paradigm of using robotic surgery in the clinical work up of these cases $(12,13)$.

Successful TORS for laryngeal neoplasms has been mainly restricted to involve supraglottic lesions, which was also observed in the present series. Supraglottic laryngectomy has become one of the standard TORS approaches and several reports have suggested expanding the use of robotic surgery in the management of laryngeal cancer (14). In comparison with the conventional transoral laser surgery, most TORS surgeons use monopolar diathermy although robotic laser instruments are available. Additionally, the access to larynx has been limited by technical factors (mouth retractors, collision of instruments in narrow spaces). The context of applying TORS for laryngeal surgery is now facing a new era with the first reported experiences of a novel semi-rigid operator-controlled robotic system (Medrobotics Flex system) (15). Furthermore, a recent safety and feasibility trial of the da Vinci Single Port

\section{REFERENCES}

1. Mäkitie AA, Cange HH, Hammarstedt-Nordenvall L, Gudjonsson A, Jóhannsson J, Laranne J, et al. Head and neck cancer management in the Nordic countries: an effort to harmonize treatment. Eur Arch Otorhinolaryngol. (2017) 274:2363-5. doi: 10.1007/s00405-017-4456-4
(Intuitive Surgical Inc., Sunnyvale, CA, USA) demonstrates that this flexible single-arm device is safe and feasible in performing TORS to access nasopharynx, oropharynx, larynx, and hypopharyngeal areas (16). The development of these new technologies emphasizes the need to consider centralized management of TORS in the Nordic countries.

The current setting for TORS in general clearly involves certain limitations, some of which are related to the da Vinci Surgical System i.e., instrumental and annual service costs. Nevertheless, the costs may be reduced by sharing the robotic instrument in between specialties. In high-volume centers, the set-up (docking) of robot and placement of the mouth retractor have improved quickly over time. Absence of tactile feedback and limited exposure for certain areas of the upper aerodigestive tract still call for further improvement (17). However, the increasing incidence of HPV-related oropharyngeal cancers and the observed improved outcome after surgical treatment in selected cases form a platform for further development of TORS (18). These factors warrant continuous actions in terms of system development and will obviously change the scene for TORS in the future.

We conclude, that a high volume of surgeries at each TORS center in the Nordic countries is needed to maintain a sufficient level of expertise and quality assurance. We also want to emphasize the potential life-threatening complications that are related to TORS and need to be considered in the learning curve phase at each center. Five out of the ten centers in the present survey reported having experienced major postoperative bleeding episodes (data not shown). Therefore, this study has value in describing the current status with TORS in the Nordic countries. Importantly, it also forms the initial steps in creating a forum between TORS surgeons at the respective centers, which is needed to design multicentre studies. Finally, it emphasizes the need for future reflections on where we are headed in terms of TORS in the field of head and neck surgery.

\section{AUTHOR CONTRIBUTIONS}

All authors (AM, HK-S, MM-L, LB, PK, TE, KS, GL, MvB, JN, PW, LG, LN, TK, CG, OR, HIC, NR, CvB) contributed to the data collection, AM prepared the first version of the manuscript and all authors commented on it and approved the final version.

\section{ACKNOWLEDGMENTS}

This study was supported by the Helsinki University Hospital Research Fund (TYH2017232), the Karolinska University Hospital Research Fund (ALF 20160596) and the Finska Läkaresällskapet.

2. O'Malley BW, Weinstein GS, Snyder W, Hockstein NG. Transoral robotic surgery (TORS) for base of tongue neoplasms. Laryngoscope (2006) 116:1465-72.

3. Weinstein GS, O’Malley BW, Snyder W, Sherman E, Quon H. Transoral Robotic Surgery: radical tonsillectomy. Arch Otolaryngol Head Neck Surg. (2007) 133:1220-6. doi: 10.1001/archotol.133.12.1220 
4. Chen MM, Roman SA, Kraus DH, Sosa JA, Judson BL. Transoral robotic surgery: a population-level analysis. Otolaryngol Head Neck Surg. (2014) 150:968-75. doi: 10.1177/0194599814525747

5. Cracchiolo JR, Roman BR, Kutler DI, Kuhel WI, Cohen MA (2016). Adoption of transoral robotic surgery compared with other surgical modalities for treatment of oropharyngeal squamous cell carcinoma. J. Surg. Oncol. 114:40511. doi: 10.1002/jso.24353

6. Rudmik L, An W, Livingstone D, Matthews W, Seikaly H, Scrimger R, et al. Making a case for high-volume robotic surgery centers: a cost-effectiveness analysis of transoral robotic surgery. J Surg Oncol. (2015) 112:155-63. doi: $10.1002 /$ jso. 23974

7. van der Sluis PC, Schreuder HW, Merks BT, Boeken Kruger AE, Verheijen R, van Hillegersberg R. Centralization of robotic surgery: better results and cost savings. Ned Tijdschr Geneeskd. (2013) 157:5228.

8. Chan JK, Gardner AB, Taylor K, Blansit K, Thompson CA, Brooks R, et al. (2015). The centralization of robotic surgery in high-volume centers for endometrial cancer patients - a study of 6560 cases in the U.S. Gynecol. Oncol. 138:128-32. doi: 10.1016/j.ygyno.2015.04.031

9. Khandwala YS, Jeong IG, Kim JH, Han DH, Li S, Wang Y, et al. The impact of surgeon volume on perioperative outcomes and cost for patients receiving robotic partial nephrectomy. J Endourol. (2017) 31:851-7. doi: 10.1089/end.2017.0207

10. Bastawrous A, Baer C, Rashidi C, Neighorn C. Higher robotic colorectal surgery volume improves outcomes. Am J Surg. (2018) 215:874-8. doi: 10.1016/j.amjsurg.2018.01.042

11. Rubek N, Channir HI, Charabi BW, Lajer CB, Kiss K, Nielsen HU, et al. Primary transoral robotic surgery with concurrent neck dissection for early stage oropharyngeal squamous cell carcinoma implemented at a Danish head and neck cancer center: a phase II trial on feasibility and tumour margin status. Eur Arch Otorhinolaryngol. (2017) 274:2229-37. doi: 10.1007/s00405-016-4433-3

12. Abuzeid WM, Bradford CR, Divi V. Transoral robotic biopsy of the tongue base: A novel paradigm in the evaluation of unknown primary tumors of the head and neck. Head Neck. (2013) 35:126-30. doi: 10.1002/hed.21968
13. Channir HI, Rubek N, Nielsen HU, Kiss K, Charabi BW, Lajer CB, et al. Transoral robotic surgery for the management of head and neck squamous cell carcinoma of unknown primary. Acta Otolaryngol. (2015) 135:1051-7. doi: 10.3109/00016489.2015.1052983

14. Bekeny JR, Ozer E. Transoral robotic surgery frontiers. World J Otorhinolaryngology Head Neck Surg. (2016) 2:130-5. doi: 10.1016/ j.wjorl.2016.05.001

15. Remacle M, Prasad VMN. Preliminary experience in transoral laryngeal surgery with a flexible robotic system for benign lesions of the vocal folds. Eur Arch Otorhinolaryngol. (2018) 275:761-5. doi: 10.1007/s00405-018-4900-0

16. Chan JYK, Wong EWY, Tsang RK, Holsinger FC, Tong MCF, Chiu PWY, et al. Early results of a safety and feasibility clinical trial of a novel singleport flexible robot for transoral robotic surgery. Eur. Arch. Otorhinolaryngol. (2017) 274:3993-6. doi: 10.1007/s00405-017-4729-y

17. Lombard B, Céruse, P. Robotics and Digital Guidance in ENT - H\&N Surgery. Elsevier Masson SAS. (2017).

18. Jouhi L, Halme E, Irjala H, Saarilahti K, Koivunen P, Pukkila M, et al. Epidemiological and treatment-related factors contribute to improved outcome of oropharyngeal squamous cell carcinoma in Finland. Acta Oncol (2017) 17:1-11. doi: 10.1080/0284186X.2017.1400688

Conflict of Interest Statement: The authors declare that the research was conducted in the absence of any commercial or financial relationships that could be construed as a potential conflict of interest.

Copyright (c) 2018 Mäkitie, Keski-Säntti, Markkanen-Leppänen, Bäck, Koivunen, Ekberg, Sandström, Laurell, von Beckerath, Nilsson, Wahlberg, Greiff, Norberg Spaak, Kjoergaard, Godballe, Rikardsen, Channir, Rubek and von Buchwald. This is an open-access article distributed under the terms of the Creative Commons Attribution License (CC BY). The use, distribution or reproduction in other forums is permitted, provided the original author(s) and the copyright owner(s) are credited and that the original publication in this journal is cited, in accordance with accepted academic practice. No use, distribution or reproduction is permitted which does not comply with these terms. 


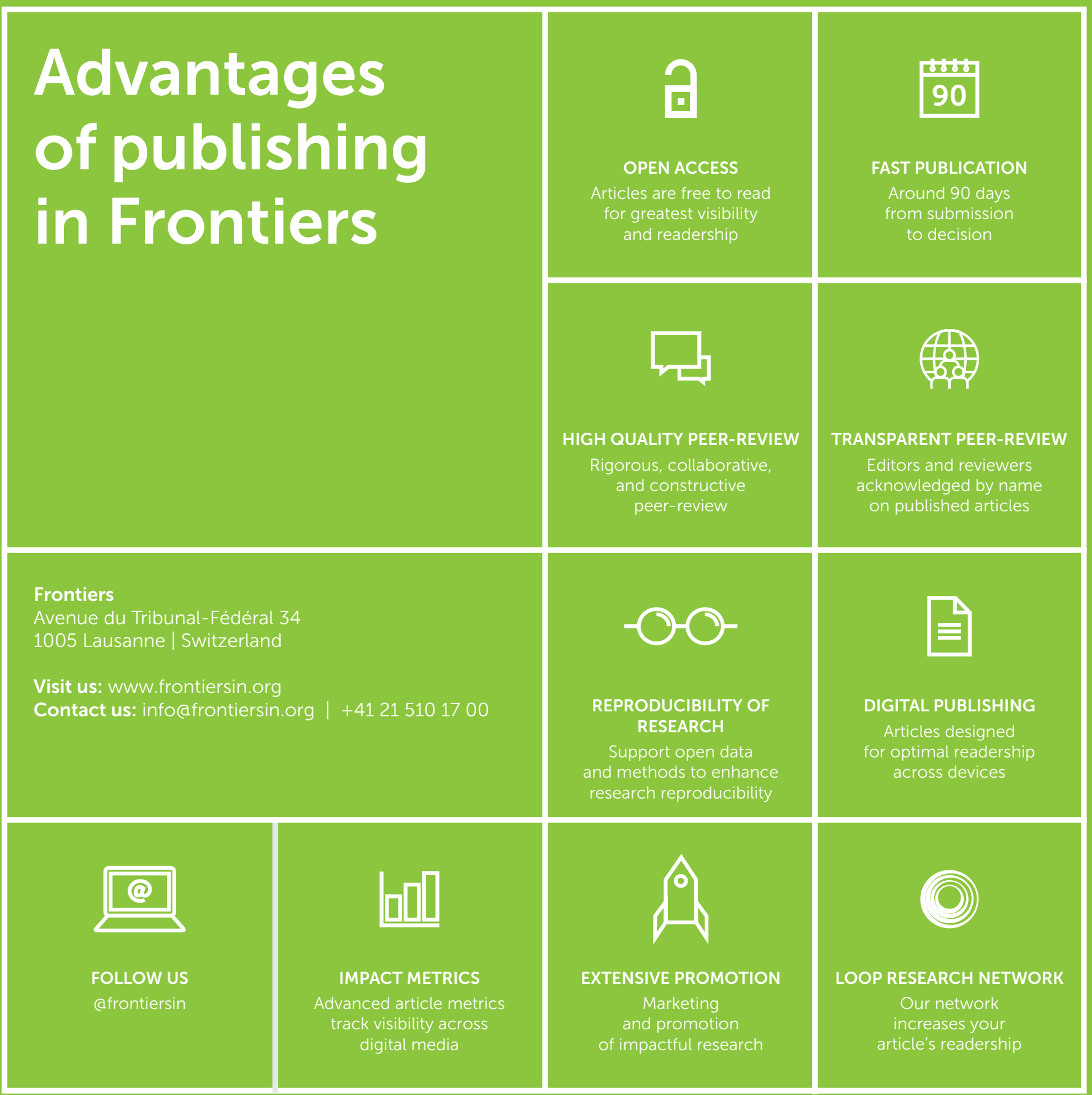

